

\section{Multilingualism across the Lifespan}

This innovative collection examines key questions on language diversity and multilingualism running through contemporary debates in psycholinguistics and sociolinguistics.

Reinforcing interdisciplinary conversations on these themes, each chapter is co-authored by two different researchers, often those who have not written together before. The combined effect is a volume showcasing unique and dynamic perspectives on such topics as multilingualism across the lifespan, bilingual acquisition, family language policy, language and ageing, language shift, language and identity, and multilingualism and language impairment. The book builds on Elizabeth Lanza's pioneering work on multilingualism across the lifespan, bringing together cutting-edge research exploring multilingualism as an evolving phenomenon at landmarks in individuals', families', and communities' lives. Taken together, the book offers a rich portrait of the different facets of multilingualism as a lived reality for individuals, families, and communities.

This ground-breaking volume will be of particular interest to students and scholars in multilingualism, applied linguistics, sociolinguistics, and psycholinguistics.

Unn Røyneland is Professor of Scandinavian Linguistics and Deputy Director of the Center for Multilingualism in Society across the Lifespan at the University of Oslo, Norway.

Robert Blackwood is Professor of French Sociolinguistics in the Department of Modern Languages and Cultures at the University of Liverpool, UK. 


\section{Routledge Critical Studies in Multilingualism}

Edited by Marilyn Martin-Jones, MOSAIC Centre for

Research on Multilingualism, University of Birmingham, UK and Joan Pujolar Cos, Universitat Oberta de Catalunya, Spain

Titles include:

21 The Aesthetics and Politics of Linguistic Borders

Multilingualism in Northern European Literature

Edited by Heidi Grönstrand, Markus Huss and Ralf Kauranen

22 New Speakers of Irish in the Global Context

New Revival?

Bernadette O'Rourke and John Walsh

23 Decolonizing Language Learning, Decolonizing Research

A Critical Ethnographic Study in a Mexican University

Colette Despagne

24 Language, Global Mobilities, Blue-Collar Workers

and Blue-Collar Workplaces

Edited by Kellie Gonçalves and Helen Kelly-Holmes

25 Sign Languages and Linguistic Citizenship

A Critical Ethnographic Study of the Yangon Deaf Community

Ellen Foote

26 A Sociolinguistics of the South

Edited by Kathleen Heugh, Christopher Stroud,

Kerry Taylor-Leech and Peter I. De Costa

27 Multilingualism across the Lifespan

Edited by Unn Røyneland and Robert Blackwood

28 Spaces of Multilingualism

Edited by Robert Blackwood and Unn Røyneland

For more information about this series, please visit: https://www.routledge. com/Routledge-Critical-Studies-in-Multilingualism/book-series/RCSM09 


\title{
Multilingualism across the Lifespan
}

\author{
Edited by Unn Røyneland \\ and Robert Blackwood
}


First published 2022

by Routledge

605 Third Avenue, New York, NY 10158

and by Routledge

2 Park Square, Milton Park, Abingdon, Oxon, OX14 4RN

Routledge is an imprint of the Taylor \& Francis Group, an informa business

(C) 2022 selection and editorial matter, Unn Røyneland and Robert Blackwood; individual chapters, the contributors

The right of Unn Røyneland and Robert Blackwood to be identified as the authors of the editorial material, and of the authors for their individual chapters, has been asserted in accordance with sections 77 and 78 of the Copyright, Designs and Patents Act 1988.

The Open Access version of this book, available at www.taylorfrancis. com, has been made available under a Creative Commons AttributionNon Commercial-No Derivatives 4.0 license.

Trademark notice: Product or corporate names may be trademarks or registered trademarks, and are used only for identification and explanation without intent to infringe.

Library of Congress Cataloging-in-Publication Data A catalog record for this title has been requested

ISBN: 978-0-367-64682-0 (hbk)

ISBN: 978-0-367-64686-8 (pbk)

ISBN: 978-1-003-12581-5 (ebk)

DOI: $10.4324 / 9781003125815$

Typeset in Sabon

by Deanta Global Publishing Services, Chennai, India 
For Elizabeth 


\section{Contents}

List of Illustrations $\quad$ ix

List of Contributors $\quad \mathrm{x}$

Preface xvi

Acknowledgements xviii

Introduction 1

UNN RØYNELAND AND ROBERT BLACKWOOD

\section{PART I}

Bilingual Acquisition: Methodological and Theoretical Advances 9

1 Child Language Assessment across Different Multilingual Contexts: Insights and Challenges from South and North HANNE GRAM SIMONSEN AND FRENETTE SOUTHWOOD

2 Developmental Perspectives on Parents' Use of Discourse Strategies with Bilingual Children

ANNICK DE HOUWER AND JANICE NAKAMURA

3 The Role of the Input in the Acquisition of Code-Switching 56 SHANNON PHILLIPS AND MARGARET DEUCHAR

PART II

Language Practices and Policies in the Family

4 Language Development, Discourse, and Politics: Family Language Policy Foundations and Current Directions 
viii Contents

5 Family Time(s): Migrant Temporalities in Family Language Planning in the Urban African South

CHRISTINE ANTHONISSEN AND CHRISTOPHER STROUD

6 From "Civilising Missions" to Indigenous Language

Reclamation: Language Policy, Language Shift, and

Maintenance in Australia and Norway

PIA LANE AND GILLIAN WIGGLESWORTH

PART III

Bilingual Ageing: Communication and Cognitive Impairments 145

7 Where Have All My Languages Gone? Aging and the Changing Multilingual Linguistic Ecology

NG BEE CHIN AND FRANCESCO CAVALLARO

8 Fostering Storytelling by Persons with Dementia in Multiparty Conversation

JAN SVENNEVIG AND HEIDI E. HAMILTON

9 Assessment Challenges in Acquired Aphasia in Multilingual Individuals

MONICA NORVIK AND MIRA GORAL

10 Pathological Language-Switching/Mixing and Its

Relationship to Domain-General Cognitive Control

VALANTIS FYNDANIS AND MINNA LEHTONEN

PART IV

Concluding Remarks

11 Carving New Spaces in the Study of Individual Multilingualism across the Lifespan

LOURDES ORTEGA

Index 


\section{Illustrations}

\section{Figures}

2.1 Lanza's (1992, 1997b) bilingual family interaction model (BIFIM)

3.1 Matrix language distribution in child mixed utterances

3.2 Matrix language distribution in individual child mixed utterances

3.3 Matrix language distribution in Siarad adult corpus compared with child mixed utterances in CIG1

3.4 Matrix language distribution in mixed utterances by Dewi's adult interlocutors compared with child mixed utterances

3.5 Matrix language distribution in child mixed utterances comparing Analysis 1 and Analysis 2

7.1 Signs outside a lift in a hospital

7.2 Sign at a hospital lobby

8.1 Throwing gesture

\section{Tables}

2.1 Studies of Parental Discourse Strategies in Dyadic Parent-Child Interactions With BFLA Children

2.2 Bilingual Parenting Resources Reviewed for Reference to Lanza (1992, 1997b) and/or BIFIM

3.1 CIG1 Child Speakers and Ages

3.2 Child Mixed Utterances in CIG1

7.1 Participants

9.1 Results of Assessment for P1 and P2

10.1 Summary of the Reviewed Studies, Reporting Whether the Language-Switching/Mixing Behaviour Results in Communication Breakdown, Participants' Performance on Cognitive Tests, and Lesion Site 


\section{Contributors}

Christine Anthonissen is Professor in General Linguistics, Stellenbosch University, South Africa. Her research focuses on discourse studies, critical discourse analysis, and sociolinguistic aspects of bilingualism and multilingualism. She is a member of the editorial board of Multilingual Margins (eds. Christopher Stroud, Quentin Williams) and on the advisory board for the series of Discourse Approaches to Politics, Society and Culture (DAPSAC). She is the author and co-author of a number of publications, including Autobiographical Narrative of Traumatic Experience: Disruption and Resilience in South African Truth Commission Testimonies (2020), and Language-in-Education Policy in South Africa: Managing the Multilingualism of Learners in the 21st century (with Anneke Potgieter, 2014).

Robert Blackwood is Professor of French Sociolinguistics at the University of Liverpool, UK, and currently editor of the journal Linguistic Landscape with Elana Shohamy. He is the author of a number of articles and book chapters on language policy and regional language revitalisation in France, including work on the linguistic landscape. He is the co-author of The Linguistic Landscape of the Mediterranean: French and Italian Coastal Cities (with Stefania Tufi, 2015) and co-editor of Multilingual Memories: Monuments, Museums and the Linguistic Landscape (with John Macalister, 2020).

Francesco Cavallaro is Associate Professor in the Division of Linguistics and Multilingual Studies at Nanyang Technological University, Singapore. His research interests are in sociolinguistics and the social aspects of bilingualism, especially of minority groups in multilingual contexts. $\mathrm{He}$ has published on language maintenance and shift, the demographics of the Italian community in Australia, language attitudes in Singapore, language and ageing, and minority groups in South East Asia. He is the author of the book Transgenerational Language Shift: From Sicilian and Italian to Australian English. 
$\mathrm{Ng}$ Bee Chin works in the area of bilingualism and multilingualism with a focus on the impact of language contact on individuals and the community they live in. Her research approach is to explore both cognitive and social aspects of language use. Currently, she is working on language and emotion in multilinguals, language attitudes, identity and social categorisation, and communicative aspects of ageing. A common thread running through her research is the connection between the self and the social. The self (person) and the community is intricately bound, and to fully understand language use we need to understand the communities we live in. Invariably, our social interactions will have an impact on the way information is organised cognitively, and $\mathrm{Ng}$ continues to navigate the nexus between these two fields. She is currently Associate Professor in Linguistics and Associate Dean of Graduate Studies in the College of Humanities and Social Sciences, Nanyang Technological University, Singapore.

Xiao Lan Curdt-Christiansen is Professor in Applied Linguistics at the Department of Education, University of Bath, UK. Her research interests encompass ideological, socio-cognitive, and policy perspectives on children's multilingual education and biliteracy development. She has examined bi/multilingual community-home-school contexts in the UK, Canada, France, and Singapore on topics of curriculum policy, language-in-educational policy, and family language policy. Her most recent research project is entitled Family Language Policy: A Multi-Level Investigation of Multilingual Practices in Transnational Families, funded by The Economic and Social Research Council (ESRC).

Annick De Houwer's pioneering research on bilingual children's language development has been highly influential. In addition to many journal articles and book chapters, she has published four books on bilingual children (two with Cambridge University Press and two with Multilingual Matters), the latest of which is entitled Bilingual Development in Childhood. She is the co-editor (with Lourdes Ortega) of the Cambridge Handbook of Bilingualism. Dr De Houwer directs the Harmonious Bilingualism Network (www.habilnet.org). HaBilNet aims to support and stimulate scientific research into harmonious bilingualism and to make sure the results of that research are widely disseminated among families and educators.

Margaret Deuchar is Emeritus Professor in Linguistics at Bangor University and Affiliated Lecturer in Theoretical and Applied Linguistics, University of Cambridge, UK. She did her PhD on British Sign Language and later worked on the bilingual acquisition, publishing Bilingual Acquisition: Theoretical Implications of a Case Study (with S. Quay, 
2000). In 2007 she became the founding director of the ESRC Centre for Research on Bilingualism at Bangor University and co-ordinated the collection and analysis of code-switching data from three bilingual communities. Analysis of the Welsh/English data collected is reported in Deuchar, Webb-Davies, and Donnelly, Building and Using the Siarad Corpus (2018).

Valantis Fyndanis is Assistant Professor of Psycholinguistics/ Neurolinguistics at the Cyprus University of Technology, Cyprus, and Researcher at the Center of Multilingualism in Society across the Lifespan (MultiLing) at the University of Oslo, Norway. He is the author of a number of articles on aphasia and related adult neurogenic disorders. Valantis Fyndanis has been Marie Curie Postdoctoral Fellow (funded by the European Commission) at the University of Potsdam, Germany. He has also held research or teaching positions at other universities in Greece, Italy, and the United States. Currently, he leads the Machine Learning Aphasia project, funded by the Research Council of Norway.

Mira Goral is Professor of Speech-Language-Hearing Sciences at Lehman College and the Graduate Center of The City University of New York (CUNY), USA. She is also Research Professor at The Center for Multilingualism in Society across the Lifespan, the University of Oslo, Norway, and Adjunct Research Professor at NYU School of Medicine. She completed her BA in Linguistics at Tel Aviv University and her PhD in Neurolinguistics at the Graduate Center, CUNY. She has published in the areas of bilingualism, multilingualism, aphasia, language attrition, and language and cognition in ageing.

Heidi E. Hamilton is Professor of Interactional Sociolinguistics in the Department of Linguistics, Georgetown University, Washington, DC, USA, where she has explored interrelationships between discourse and health in a wide range of interdisciplinary collaborations since 1990. Her recent books are Language, Dementia and Meaning Making: Navigating Challenges of Cognition and Face in Everyday Life (2019), the Wiley-Blackwell Handbook of Discourse Analysis (with Tannen and Schiffrin, 2015), and the Routledge Handbook of Language and Health Communication (with Chou, 2014). Awards include Fulbright Distinguished Chair in Linguistics (Innsbruck); DAAD Gastdozentin (Berlin); and Humboldt Research Prize (Potsdam).

Kendall King is Professor of Second Language Education at the University of Minnesota, USA. Her teaching and research address language planning and policy, bilingualism and language learning, and language use and ideology. She is particularly interested in how minoritized languages can be best supported through official policies and practices at home and at school. With more than 100 publications, her work has been widely 
published, and she regularly engages in community outreach and service in schools locally, nationally, and internationally. She was an editor of the journal Language Policy and currently serves as President of the American Association of Applied Linguistics (AAAL).

Pia Lane is Professor of Multilingualism at the Center for Multilingualism in Society across the Lifespan (MultiLing) at the University of Oslo, Norway. Her research focuses on multilingualism in Northern Norway, with a particular emphasis on language policy, language shift, language revitalisation in relation to Indigenous and minoritised languages. Her publications include Standardizing Minority Languages: Competing Ideologies of Authority and Authenticity in the Global Periphery, Routledge (coedited with James Costa and Haley De Korne, 2017) and Negotiating Identities in Nordic Migrant Narratives - Crossing Borders and Telling Lives, Palgrave (co-edited with Bjørghild Kjelsvik and Annika Bøstein Myhr 2022). She is co-editor-in-chief of LME Linguistic Minorities in Europe Online (LME), published by De Gruyter.

Minna Lehtonen is Professor at the Department of Psychology and Speech Language Pathology at the University of Turku, Finland, and Professor II at the Center for Multilingualism in Society across the Lifespan at the University of Oslo, Norway. Previously, she has worked at Åbo Akademi University and the University of Helsinki in Finland, as well as the University of Maryland in the USA. She has authored a number of articles on psycho- and neurolinguistics of bilingualism. She has published on topics related to the advantages and costs of bilingualism, cognitive control in language processing and switching, and the neurocognitive basis of morphological processing.

Janice Nakamura is Associate Professor at the Department of English, Kanagawa University, Japan. She investigates child bilingualism and family language policy within the Japanese context and has worked with more than 100 intermarriage families in the past 14 years. Her research articles have appeared in journals such as the International Journal of Bilingualism and Bilingual Education, International Multilingual Research Journal, and Multilingua. Her recent work on receptive child bilingualism and monolingual children's "language regrets" draws attention to the importance of fostering children's bilingualism in the home.

Monica Norvik shares her time between clinical work with individuals with aphasia and PPA at Statped, Oslo, as a researcher at MultiLing (Center for Multilingualism in Society across the Lifespan) at the University of Oslo, and as Adjunct Associate Professor at the Norwegian University of Science and Technology, Trondheim, Norway. Her main academic interests include clinical linguistics, in particular, language disorders in adults with aphasia and dementia, assessment, and treatment of multilingual individuals, and how working with multilingual individuals with aphasia 
and dementia can inform linguistic theories on multilingual language processing. She has published in the areas of multilingualism, aphasia, assessment, and rehabilitation.

Lourdes Ortega is a Professor in the Department of Linguistics at Georgetown University, USA. She is best known for an award-winning meta-analysis of second language instruction published in 2000, a best-seller graduate-level textbook Understanding Second Language Acquisition (2009, translated into Mandarin in 2016), and since 2010 for championing a bilingual and social justice turn in her field of second language acquisition. Recent articles have appeared in CALICO Journal (2017), World Englishes (2018), Modern Language Journal (2019), and Language Learning (2020). Her latest book is The Cambridge Handbook of Bilingualism (co-edited in 2019 with Annick De Houwer). She is the general editor of Language Learning.

Shannon Phillips graduated in Linguistics from the University of Cambridge in 2019. She wrote her undergraduate dissertation on the acquisition of code-switching by Welsh/English developing bilinguals. She completed her CELTA certificate to qualify as an ESOL teacher in 2019 and is currently teaching English to asylum seekers in the United Kingdom and to speakers of Chinese. She is interested in bilingual development and language acquisition.

Unn Røyneland is Professor of Scandinavian Linguistics and Deputy Director at the Center for Multilingualism in Society across the Lifespan (MultiLing) at the University of Oslo, Norway. Her research focuses on language practices on social media and among adolescents in urban multilingual environments, dialect, and migration, and language attitudes, policy, and advocacy. Her recent publications include Multilingual Youth Practices in Computer Mediated Communication (co-edited with Cecelia Cutler, 2018) and a special issue of the Journal of Multilingual and Multicultural Development on "Migration and Dialect Acquisition in Europe" (co-edited with Peter Auer, 2020). She is co-editor-in-chief of Linguistic Minorities in Europe Online (LME), published by De Gruyter.

Hanne Gram Simonsen is Professor Emeritus of Linguistics at MultiLing, Department of Linguistics and Scandinavian Studies, University of Oslo, Norway. Her research covers language development across the lifespan from childhood to old age, including clinical linguistics. Her publications on child language include works on the Norwegian adaptation of the MacArthur-Bates CDI (2012, 2013, 2014) and a special issue of Clinical Linguistics and Phonetics (co-edited with Ewa Haman on the assessment tool Cross-linguistic Lexical Tasks, 2017). At the other end of the lifespan, a recent publication is Multilingualism and Ageing: An Overview (co-edited with Kees de Bot and Charlotta Plejert, 2020). 
Frenette Southwood is Associate Professor of Linguistics at Stellenbosch University, South Africa. She has authored a number of articles on language impairment in Afrikaans-speaking children and on culturally and linguistically fair child language assessment. Together with Ondene van Dulm, she is adapting the Diagnostic Evaluation of Language Variation (co-authored by Harry Seymour, Tom Roeper, Jill de Villiers, Peter de Villiers, and Barbara Zurer Pearson) for Afrikaans and South African English, and she is a member of the team developing the MacArthurBates Communicative Development Inventory for South Africa's 11 official languages.

Christopher Stroud is Emeritus Senior Professor of Linguistics at the University of the Western Cape, South Africa. He is also Professor of Transnational Multilingualism at Stockholm University, Centre for Research on Bilingualism, Sweden. He has worked on multilingualism and literacy in a variety of contexts, including South Africa, Mozambique, Papua New Guinea, Sweden, and Singapore. His current research focuses on practices and ideologies of multilingualism in Southern Africa, specifically Linguistic Citizenship, as a way of rethinking the role of language in brokering diversity in a decolonial framework.

Jan Svennevig is Professor of Language and Communication at Agder University and MultiLing, University of Oslo, Norway. His research deals with workplace interaction, L2 conversations, pragmatic particles, and communication in dementia. Recent publications include "Pre-empting understanding problems in L1/L2 conversations: Evidence of effectiveness from simulated emergency calls" (Applied Linguistics, 2019, with J. Gerwing, B. U. Jensen, and M. Allison) and "Accounting for forgetfulness in dementia interaction" (Linguistics Vanguard, 2019, with A. M. Landmark).

Gillian Wigglesworth a Redmond Barry Distinguished Professor in the School of Languages and Linguistics at the University of Melbourne, Australia and chief investigator in the ARC Centre of Excellence for the Dynamics of Language, Australia. Her major research focus is on the languages Australia Indigenous children living in remote communities are learning, and the linguistic challenges the children face as they enter the formal, usually English-based, school system. Her most recent book, From Home to School: Language Practices of Indigenous Children and Youth, Palgrave (co-edited with Jane Simpson and Jill Vaughan, 2018) documents much of this work. 


\title{
Preface
}

\author{
Marilyn Martin-Jones
}

The first steps towards incorporating this innovative Festschrift project into the Routledge series on Critical Studies in Multilingualism were taken in a meeting with Unn Røyneland, at the Center for Multilingualism in Society across the Lifespan (The MultiLing Center), at the University of Oslo, in November 2019. I later learned, through correspondence with Robert Blackwood and Unn Røyneland, that the essential groundwork for the project was actually laid during a month-long visit by Robert to the MultiLing Center in May 2018. After almost three years of collaboration between contributors and dedicated editorial work by Robert and Unn, two landmark publications in the field of multilingualism are ready to go into production at the beginning of March 2021. For me and for Joan Pujolar, my co-editor, it is a pleasure and a privilege to be able to include these volumes in the series. I say this for three reasons:

First, because the volumes have been designed and edited by Robert and Unn, with both vision and dedication, as an international tribute to Elizabeth Lanza, as she retires from the leadership of the MultiLing Center. Throughout her academic career, Elizabeth has shaped the contours of several different strands of research on multilingualism in distinctive ways: through her outstanding research innovation and strategic crossing of disciplinary boundaries; through her exceptional capacity for sustained and highly productive research collaboration with scholars in the global north and, more recently, in the global south; and through her inspired leadership and vision for the MultiLing Center, which has provided a unique forum for ground-breaking research in the sociolinguistics and psycholinguistics of multilingualism. These dimensions of Elizabeth's scholarly achievements are alluded to, again and again, across the chapters of these two volumes.

Second, Joan Pujolar and I warmly welcome the inclusion of these two volumes in our Routledge series because of the transdisciplinarity reflected in them and because of the illuminating ways in which they consider the trajectories of speakers and language resources across the lifespan and across social and institutional spaces. This transdisciplinarity fits well with the way in which we have conceptualised the book series, keeping it open to wider debates in the social sciences and the humanities. 
Third, we welcome the original ways in which Robert and Unn have designed and edited the two volumes. They have re-imagined the genre of Festschrift in bold and novel ways. The overall organisation of the two volumes around different themes makes it possible to index different moments in Elizabeth's career as a researcher, along with the different fields of research to which she has made major contributions. Moreover, all of the chapters are co-authored, with a view to echoing Elizabeth's commitment to research collaboration, in her own work and in the research developed in the MultiLing Center.

Forty-four scholars from across the world have contributed to these two volumes. They are scholars working at the cutting edge of different strands of research into multilingualism, and they include established and early career researchers. Volume I, entitled Multilingualim across the Lifespan, showcases psycholinguistic and sociolinguistic research that addresses issues similar to those that Elizabeth has been concerned with in her work on bilingual acquisition and on the language practices and policies of bilingual families. This volume also incorporates a section on bilingual ageing, which has close resonances with research of this type developed within the MultiLing Center. Volume II, entitled Spaces of Multilingualism, presents empirical work and theory-building in the sociolinguistics of multilingualism, focusing primarily on areas of research where Elizabeth has herself made major contributions - areas such as the study of interaction and the construction of identities, and research into linguistic landscapes, in global south and global north settings. This volume also points to ongoing shifts in thinking about the contexts for research into language policy-making and for language pedagogy relating to linguistic landscapes. The range, depth and quality of the scholarship assembled here by Robert and Unn are impressive. Together, these two volumes make the most-fitting tribute to Elizabeth Lanza's outstanding achievements as a researcher, and latterly, as the Director of the MultiLing Center at the University of Oslo, Norway. 


\section{Acknowledgements}

The journey that culminates in these volumes began in Bern at XScapes, the tenth International Linguistic Landscape Workshop (2018), and we would like to use this space to express our gratitude to those who have supported this project on its road to completion.

In particular, we would like to thank the Center for Multilingualism in Society across the Lifespan (MultiLing) for its generous financial support for this project, especially its funding for Open Access to ensure that the work contained here can be shared as widely as possible. Equally, the Center supported Robert in a month-long visiting scholarship at MultiLing, during which the groundwork for the project was laid. Also at MultiLing, we would like to thank research assistant Anne Marte Haug Olstad for her careful copy-editing of all the chapters. Hence, this volume was partly supported by the Research Council of Norway through its Centres of Excellence funding scheme, project number 223265.

We record here our gratitude to the Department of Languages, Cultures, and Film at the University of Liverpool, UK. Unn came to Liverpool to give a research paper to the Place, Space \& Belonging Research Group in May 2019 , and we used this opportunity to work together on this project.

We are delighted that these volumes appear in the Routledge series Critical Studies in Multilingualism, and we would like to thank Marilyn Martin-Jones for her input on the proposal and for her subsequent encouragement. At the same time, we are grateful to Joan Pujolar for supporting us and this endeavour. Elysse Preposi has guided us and answered our questions over the lifespan of this entire project, and we thank her here for her patience and enthusiasm.

Academic scholarship is always improved by peer review, and as well as inviting some of the contributors to review critically the chapters here, we also called on expertise from across our disciplines. In particular, therefore, we would like to thank Peter Auer, Jennifer Gerwing, Rafael Lomeu Gomez, 
Ewa Haman, Lars-Christer Hydén, Haley De Korne, Julien Mayor, Satomi Mishina-Mori, Loraine Obler, Åsa Palviainen, and Eleonora Rossi.

As with all such projects, we are supported invisibly but unfailingly by our families who have endured us spending hours in Zoom meetings. Robert would like to thank explicitly Jude, Luke, and Julia, and Unn would like to thank Bjørn, Hulda, Tollef, and Max. 


\section{Introduction}

\section{Unn Røyneland and Robert Blackwood}

This is the companion volume to Spaces of Multilingualism. Together these two collections pay tribute to our colleague and friend, Elizabeth Lanza, a towering figure across a range of disciplines whose research interests inspire and underpin the philosophy of this homage. In planning these volumes, we agreed that one of the guiding principles would be that every chapter is coauthored, articulating in their conception and execution one of Elizabeth's approaches to life and scholarship, which we seek to honour. Not just through her leadership of the Center for Multilingualism in Society across the Lifespan (MultiLing) but throughout her career, Elizabeth has invited, engineered, and nourished collaborations between researchers. This recognition and valuing of the power of working together has come to characterise the work of MultiLing, the research center financed by the Research Council of Norway as a Center of Excellence. To reflect this approach, all of the contributors to both volumes were asked to work with someone else, in some cases where the authors have not collaborated before. These partnerships characterise, in their method, the creativity that can be unexpected, unorthodox, and even unlikely, but their outcomes are much more than the sum of their parts. As such, these volumes harness the imagination and dynamism of a wide range of researchers, both established and early-career, and exemplify the enthusiastic and creative relationships that have been initiated and nourished by Elizabeth over her own academic lifespan. Equally, they go some way to reflecting the numerous examples of co-authorship that have come to define Elizabeth's approach to scholarship where she has coauthored and co-edited with more than two dozen collaborators.

\section{Multilingualism and Linguistic Diversity}

In positioning the two volumes around multilingualism and linguistic diversity more generally, we address here one of the most pressing human issues: an issue that has long been recognised and cherished in some parts of the world, taken for granted elsewhere, and positively resisted and decried in other places (often in those countries - for there is a national dimension to this - which self-identify as modern, successful, and welcoming). Thinking 


\section{Unn Røyneland and Robert Blackwood}

through linguistic diversity and multilingualism as a phenomenon is one of the aims of these two volumes; in particular, the authors interrogate some of the easy caricatures of what it means to deal with linguistic diversity: to be multilingual, to live multilingually, to organise others' lives multilingually. Assuming that multilingualism equates with multiple languages, some might think that we are overlooking the complexity contained within this axiom. We seek, however, to grapple with the breadth of questions surrounding the interaction between, across, and via languages and lects.

Languages and lects are acquired, deployed, restricted, encouraged, communicated, displayed, (re)created, and lost across the lifespan. The trajectories of languages - and here we deliberately (and for some provocatively) uncouple the concept of a language from habitual understandings in traditional discussions of acquisition - are not linear, regular, or without disruption. In this volume, we explore these trajectories as they play out in different settings across the lifespan, engaging creatively and challengingly with questions that have long been addressed within psycholinguistics and sociolinguistics, including language acquisition, family language practices and policies, and the consequences of aphasia and dementia on language use. From discussing approaches to understanding parental influence on bilingualism in children, multilingual family interactions, and ageing in a multilingual environment, through to analysing the impact of medical conditions (such as a stroke) and brain diseases (aphasia and dementia) on multilingual individuals, the authors foreground new research, unified by the shared interest in contemporary debates on multilingualism. This volume, alongside its partner volume dedicated to spaces of multilingualism, tackles issues around the processes of change in and of languages and societies. Grounded in cutting-edge research from scholars from across the world, the volume interrogates multilingualism as an evolving phenomenon at landmarks in individuals', families', and communities' lives.

\section{The Volume and Elizabeth's Work}

We have arranged this volume into three parts, each of which engages with a different perspective of Elizabeth's work. The boundary between the two volumes is not fixed, and in organising them, we have discussed at length how best to sequence the contributions. Consequently, it is in some ways unhelpful to characterise the volumes according to their intellectual orientation, especially as a number of chapters could sit in either tome. Nevertheless, this volume leans towards the psycholinguistic, whilst its companion volume tends towards the sociolinguistic - although, from the outset, we recognise the problematic nature of categorising each volume as such, especially as we argue that the symbiosis of these two areas of linguistics is highly productive and - of course - is embodied in Elizabeth's career.

Notwithstanding, we have structured this volume into three parts, the first of which addresses the methodological and theoretical advances within 
the field of bilingual acquisition, "Bilingual Acquisition - Methodological and Theoretical Advances" (Part I). The three contributions in this section make very clear the foundational impact Elisabeth's work has had on the study of bilingual children and parent-child interaction. Her bilingual family interaction model for conversation analysis between bilingual parents and their bilingual children (Lanza 1990, 1997) is discussed at length. One of Elisabeth's first publications, from 1988 and published by Multilingual Matters, addressed infant bilingualism and language strategies in the home. Infant bilingualism and the importance of the family were also central topics in her PhD from (1990), a work which was the basis for the monograph published by Oxford University Press under the title Language Mixing in Infant Bilingualism: A Sociolinguistic Perspective (1997, paperback edition with new afterword 2004).

In the second part of this volume, three contributions deal with language practices and policies in the family, "Language Practices and Policies in the Family" (Part II). These chapters discuss the very foundations of the field as well as current directions, family language policy and planning in the contexts of both migration and indigenous language reclamation. The study of family language policy (FLP) has been a central part of Elizabeth's research throughout her career. As we have already mentioned, the focus on families was prominent both in her early work on bilingual children and more recently through the MultiFam project: "Family Language Policy in Multilingual Transcultural Families" (2015-2018) led by Elizabeth and funded by the Research Council of Norway. Notably, Elizabeth has co-edited no less than four seminal special issues on multilingual family language policy: one with Li Wei in Journal of Multilingual and Multicultural Development (2016), one with Kendall King International Journal of Bilingualism (2017), and two with Xiao Lan Curdt-Christensen in International Journal of Multilingualism and in Multilingua Journal of Cross-Cultural and Interlanguage Communication (both 2018). In her blog post in Psychology Today from January 2021, Elizabeth asks rhetorically "Family Language Policies: Do We Need Them?" Taking off from her own journey towards multilingualism in childhood when she and her family sailed into New York Harbor in the 1950s, arriving from across the Atlantic, Elizabeth discusses recent research in FLP and gives advice to families who want to raise their children bi- or multilingually.

The third part discusses multilingual ageing, communication, and cognitive impairments, "Bilingual Ageing - Communication and Cognitive Impairments" (Part III). The four contributions in this part bring in both socio- and psycholinguistic perspectives to the study of multilingualism in the elderly population. The chapters deal with communication hurdles in healthcare settings; communication strategies to overcome isolation and support high-quality social participation of people with dementia; challenges in diagnosing and treating multilingual people with aphasia offering a set of best practices; and last but not least how the study of multilinguals 


\section{Unn Røyneland and Robert Blackwood}

with aphasia may provide an important window into the human capacity for language. Understanding and supporting multilingual communication for the well-being of the elderly is a central topic running through all four chapters. How to secure well-being and both social and linguistic rights for all age groups and throughout the lifespan is central not only to these four chapters but also to all the chapters in this volume and, moreover, to its companion volume Spaces of Multilingualism. This is also at the very core of what Elizabeth wanted to achieve when establishing MultiLing as a Center of Excellence. The aspiration and the reality have been to bridge the gap between sociolinguistics and psycholinguistics in order to, and here we cite MultiLing's mission statement, "contribute to how society can deal with the opportunities and challenges of multilingualism through increased knowledge, promoting agency for individuals in society, and a better quality of life, no matter what linguistic and social background we have."

\section{The Chapters}

Part I of this volume returns to Lanza's first main research interest, namely bilingual acquisition, and focuses on the methodological and theoretical advances that have emerged over the last couple of decades. In the opening chapter, Simonsen and Southwood consider the disentangling of language impairment from multilingualism in assessments for children, drawing on the findings from an EU-funded project into multilingual children (Chapter 1). Recognising the multifaceted nature of child multilingualism, they interrogate the applicability and usefulness of cross-linguistic lexical tasks and a communicative development inventory in both Norway and South Africa. Focussing in particular on isiXhosa-speaking pre-schoolers, Simonsen and Southwood argue that, to be valid, assessment tools must consider local contexts and whilst there is much that can be exploited from research in the North, the variability and complexity of childhood multilingualism in the South have to be addressed. Next, De Houwer and Nakamura discuss developmental perspectives on how parents deploy a range of discourse strategies with bilingual children, considering Lanza's bilingual family interaction model for conversation analysis between bilingual parents and their bilingual children (Chapter 2). They analyse the factors which contribute to parents' use of bilingual rather than monolingual strategies in interactions with their children, highlighting the importance of parental language awareness and beliefs. De Houwer and Nakamura conclude that monolingual strategies are often hard to put into practice and may not produce lasting effects; they also draw attention to children's agency and how their strategies influence those of their parents, leading them to reaffirm the call for informing parents about the value of monolingual strategies. The third and final chapter in this part sees Phillips and Deuchar critically examine the little-studied issue of how children acquire code-switching when 
they are raised in communities where this is a common practice (Chapter 3). They discuss findings from a bilingual community in Wales, UK, where they focus in particular on mixed language utterances featuring where the matrix language was either Welsh or English and seek to discern any differences in the code-switching patterns of adults and of children. Phillips and Deuchar find that, from as early as 2 years old, children start to reproduce adult code-switching patterns when the choice of the matrix language is the same.

Part II is devoted to language policies and practices in the family, and King and Curdt-Christiansen open this part with their perspective on family language policy, with particular reference to the influence of Lanza's work on three projects: one on language socialisation in Malay and Chinese households in Singapore; a second on Chinese families in the UK; and the third on Latino families' understanding of immigration and deportation policies in the USA (Chapter 4). They posit that family language policy is coconstructed by both adults and children and that, for transnational families, societal ideologies which place little value on minority languages constitute very real obstacles to supporting multilingual development. King and CurdtChristiansen bring the role of the media and parents' understanding of highly mediated worlds into view, especially given the normalisation of technological connections with remote family members, in the quest to understand better how families create and define themselves through language. In the second chapter of this part, Anthonissen and Stroud consider the contextual determination of family language policy within migrant families to South Africa, privileging the constructs of time and temporality (Chapter 5). By considering the saliency of temporal experiences, including itineraries, provisionality, and improvised futures, they determine that particular identities and background factors interact with temporalities of migration but that the migrants they worked with did not recognise themselves as multilinguals with linguistic capital. Anthonissen and Stroud conclude that, in part because the families in their study do not fit traditional family structures and rarely contemplate language questions, their family language policies are remarkably different from those of socially secure families with time and material resources. Lane and Wigglesworth draw this part to a close with a comparison of the trajectories of language policy, shift, and maintenance with a particular focus on indigenous communities in Norway and Australia (Chapter 6). They chart the rise of the idea of the monolingual nation with its civilising mission for indigenous groups, underpinning assimilatory processes which, in both Norway and Australia, exploited educational systems. They attend to the reawakening of some Aboriginal and Torres Strait languages in Australia and the co-option of schools into the revitalisation of Sámi languages in Norway. Lane and Wigglesworth determine that indigenous languages have suffered significantly from Western ideologies that have degraded them and that revitalisation efforts are helping reclaim languages, cultural practices, and ways of knowing. 


\section{Unn Røyneland and Robert Blackwood}

Part III of this volume is dedicated to bilingual ageing, and the authors consider the questions surrounding communication and cognitive impairments. The first chapter of this part is written by $\mathrm{Ng}$ and Cavallaro, exploring limited English proficiency in the setting of healthcare, and in particular in Singapore where Linguistically Displaced Older Adults (in particular speakers of Mandarin, Cantonese, and Hokkien) struggle to communicate successfully with doctors (Chapter 7). Ng and Cavallaro highlight the various strategies deployed under these circumstances, including the use of ad hoc interpreters, the minimum threshold of "getting by," and the drawing in of family members to intercede between the patients and medical staff. The authors highlight how both patients and healthcare professionals experience language-discordant encounters and have to face the repercussions of this problem, which together point to the additional challenges of Linguistically Displaced Older Adults whose experience can be backgrounded as the spread of English has created unequal multilingualism in Singapore. The next chapter sees Svennevig and Hamilton contend with the question of how to foster storytelling - an important function for maintaining personal identity and for establishing and developing social relations with others - by Persons with Dementia (PWD) (Chapter 8). Drawing on approaches from conversation analysis and interactional sociolinguistics, the authors of this chapter provide a close analytical reading of two instances of storytelling involving at least one PWD, identifying well-intended but ultimately disruptive practices by participants in the two settings. Svennevig and Hamilton, in analysing the trajectory from relative autonomy to interdependence with others, critique discursive practices which not only support Persons with Dementia in multiparty interactions but also those, which despite participants' best intentions, can derail the storytelling, and point to advice that is invaluable to the entourages of PWD.

In their chapter, Norvik and Goral review the main challenges associated with the assessment of linguistic and communication skills in multilingual individuals who acquire aphasia, not least given the limited availability of appropriate assessment tools (Chapter 9). They note how these communication difficulties result in numerous significant challenges for individuals who acquire aphasia and go on to consider the variables in the unevenness of aphasia across the languages in the individual's repertoire. Norvik and Goral highlight the role of identity considering aphasia and draw attention to the importance of both trained interpreters for testing individuals and of culturally sensitive, standardised elicitation material, stressing the benefits of less formal assessments and a recognition of language backgrounds. The final chapter in this part is by Fyndanis and Lehtonen, who also discuss bilingual persons with aphasia but from the perspective of pathological language mixing/switching exhibited by individuals with deficient cognitive control (Chapter 10). They focus on the debate surrounding the interpretation of excessive language mixing/switching as either an issue of control deficit or a communicative strategy, drawing attention to the further challenge 
posed in cases where the examiner assessing the persons with aphasia was multilingual. They conclude that further testing of executive functions in persons with aphasia would complement more traditional neuropsychological testing and shed new light on our understanding of language control mechanisms.

Ortega draws this volume to a close with her concluding remarks, signalling how both Lanza's work and this volume carve out new spaces in the study of individual multilingualism across the lifespan (Chapter 11). Ortega attends to the linking of young bilinguals' development to discourse dynamics and socialisation practices, teasing out the wider societal perspectives. Here, she pinpoints policy, ideology, and power as three factors that are impossible to ignore in the debates around Family Language Planning. Ortega also reviews the challenges and possibilities for research into health frailties in older age as part of adults' well-being, drawing this volume to a close by saluting Lanza's almost unrivalled ability to marry the fields of psycholinguistics and sociolinguistics - a feat we seek to echo in this volume. 


\section{Part I}

\section{Bilingual Acquisition}

Methodological and

Theoretical Advances 


\title{
1 Child Language Assessment across Different Multilingual Contexts
}

\author{
Insights and Challenges \\ from South and North
}

\author{
Hanne Gram Simonsen and \\ Frenette Southwood
}

\section{Introduction}

It is well known that to obtain a fair representation of language skills in multilingual people, all their languages must be assessed (Bedore and Peña 2008). However, due to a lack of comparable tools across languages, this is not always possible. In many cases, assessment tools are only available in well-studied languages, often those with high social status or the languages of schooling. For pre-school children, being assessed only in the majority language may render misleading results if that language is not spoken in the child's home and the child has had little or no exposure to it. Regardless of whether appropriate assessment tools are available, language assessment may be important and necessary for many purposes - amongst others to allow identification of a possible language impairment or delay. In the case of multilingual children, multilingualism needs to be disentangled from language impairment, as the language of children who are multilingual may share characteristics with that of children with language impairment. For instance, similarly to children with language impairment, multilingual children might lag behind their monolingual peers - when only one of their languages is taken into account. Whereas one does not want to diagnose typically developing multilingual children as language impaired, underdiagnosing language impairment in multilingual populations, and thereby denying them access to whatever support may be available in their contexts, is detrimental to multilingual and monolingual children alike.

The lack of assessment tools for multilingual children was specifically addressed by a 2010-2013 European Union-funded network of child language researchers (COST Action IS0804, Bi-SLI, "Language Impairment in a Multilingual Society: Linguistic Patterns and the Road to Assessment," www.bi-sli.org), and their activities still continue. In this network, a collective, international effort was made to develop assessment tools for different 


\section{Hanne Gram Simonsen and Frenette Southwood}

language domains across a wide range of languages, resulting in a battery of tools under the umbrella name LITMUS (Language Impairment Testing in Multilingual Settings; see, e.g. Armon-Lotem, de Jong, and Meir 2015). Here, we will focus on vocabulary, and take as our point of departure our experience with two different tools for assessing vocabulary in multilingual children, namely the MacArthur-Bates Communicative Development Inventory (CDI) and the LITMUS Cross-Linguistic Lexical Tasks (CLT). These tools are aimed at different age groups, and they were developed in very different ways, but they both exist in linguistically and culturally comparable versions across many different languages. As such, a basic assumption is that those versions can be used for assessing the different languages of a multilingual child, and that they will indicate strengths and weaknesses in the child's languages in an accurate manner. However, as we will show, our work across different populations of multilingual children indicates that however comparable our child language tools are meant to be, they need to be used with care, and their results need to be interpreted reflectively.

One of the reasons for treating the language assessment results of multilingual children with caution is that there is no such thing as The Multilingual Child. Multilingualism, also childhood multilingualism, can take many forms (see, e.g. Butler 2013; Lanza 2007; Wei 2013). A child could, for instance, be acquiring two or more languages simultaneously (from birth) or consecutively/successively (where exposure to languages other than the first language (L1) takes place after the age of 2 years); early (before the age of 6 or 7 years) or late; and additively or subtractively, where the latter refers to the child learning the second language (L2) to the detriment of the first, as is often the case when the L1 is a minority language. The relative exposure to each language may vary, as well as where and how each language is learnt. The child could comprehend and speak the $\mathrm{L} 2$ or could be a passive multilingual who is able to understand the L2 but does not speak it. All languages could be acquired in the child's home context, or some could be acquired through exposure at the childcare institution or in the community at large. There could be a lot of community support for the child's languages or little to no such support for one or more of the languages. Such heterogeneity amongst multilingual children calls for caution when interpreting research findings and assessment results across populations and contexts.

In Norway, albeit generally considered a monolingual country, most children grow up multilingually in the sense that they are exposed to Norwegian at home, in childcare, at school, and in all spheres of society; have access to books, television shows, and other educational and entertainment artefacts in Norwegian, but start acquiring English early on through activities on the internet such as games, YouTube videos, and pop music. Some children are introduced to English in kindergarten, and all are exposed to it at school, from the age of 6 years. More than one-fifth, and maybe up to onethird, of Norwegian children are exposed to other languages in the home, having one or more parents speaking one of the traditional languages in 
Norway, such as Sámi or Kven, or more recent immigrant languages such as Polish, Somali, Swedish, or Turkish (Statistics Norway 2020a). Some of the languages have higher status in Norway than others, and the Norwegian community typically supports the acquisition of, for example, English and Swedish over Polish, Somali, and Turkish. Swedish, Danish, and Norwegian are also (near) mutually intelligible, providing a linguistic advantage to children acquiring a combination of these languages. Overall, more than $92 \%$ of children aged 1 to 5 years attend kindergarten (Statistics Norway 2020b). The age of kindergarten entry varies to some extent between cultural groups, but increasingly children start kindergarten already at the age of 1 year. The access to educational and entertainment artefacts in the various languages differ; children speaking Somali, for example, will find very little written or visual artefacts of their home language.

In South Africa, multilingualism is the norm, and childhood multilingualism takes many forms, which differ from the kinds of multilingual contexts in which Norwegian children grow up. Unlike Norway, South Africa does not have a country-wide dominant language in terms of the number of speakers. Of the country's 11 official languages, isiZulu (at 25\%) has the largest percentage of home language speakers and isiNdebele (at 1.6\%) the smallest. Although English is the lingua franca in South Africa, it is spoken as a L1 by only $8 \%$ of the population (Statistics South Africa 2018b). The majority of South African children do not receive sufficient exposure to English in their daily lives to allow them to enter school proficient in the language. Yet, English is the predominant and preferred language of education. Note that almost half of the children under the age of 6 years $(47 \%)$ do not attend childcare institutions before school entry and thus receive the vast majority of their language input at home. Home childcare is, however, not always of high quality in terms of language interaction; for instance, just over half of South African children aged 0 to 6 years are frequently read to or told stories by members of their household, and about a third of this age group are not entered into conversation with by members of their household (Statistics South Africa 2018a), thereby reducing the quantity of input received in any of their languages. The majority of South Africans have an African language as their L1, and many children grow up with several African languages in their household and/or community. They often form part of sectors of South African society that have little access to print material in their homes and communities, be it in their home languages or in English. Contrastively, for children who grow up with English and Afrikaans as their only languages, there is in theory access to many high-quality children's books, television shows, and mainstream movies. However, provision of, and access to, such resources are related to SES. In terms of family income, South Africa is one of the most unequal countries in the world, and half of South African adults live below the upper-bound poverty line (Statistics South Africa 2019). Thus, access to language stimulation resources, rather than merely their existence, is at issue in many South African communities. 


\section{Hanne Gram Simonsen and Frenette Southwood}

Given the variation in the number of languages and combination of languages, the age of first exposure and the quality and cumulative quantity of exposure to each language, amount of community support for each language, language-related expectation of the school system, and cultural and other contexts in which children acquire their language(s), the over-generalisation of research findings and assessment results should be avoided. One should also bear in mind that most of the published research findings on child multilingualism were generated in the North, in so-called WEIRD contexts (Western, Educated, Industrialised, Rich, and Democratic; see Henrich, Heine, and Norenzayan 2010). One should thus not assume that these findings will hold as is for childhood multilingualism in underresearched, majority world settings.

With these caveats in mind, we will discuss the two vocabulary assessment tools mentioned above, CDI and CLT, in light of our experiences based in Norway (an example of a WEIRD context) and South Africa (representing under-researched non-WEIRD contexts). We seek to illustrate the influence of different contexts on both their construction and their use for multilingual children, and how this offers insights, but also poses challenges to take into account.

\section{Vocabulary Assessment through Parental Report: CDI}

The MacArthur-Bates Communicative Development Inventory is a parental report tool originally developed for American English (see Fenson et al. 2007), but currently adapted into nearly 100 languages (https://mb-cdi.stanford.edu/) from a wide range of language families. It typically comes in two versions: an infant version, Words and Gestures, aimed at the age group 8 to 18 months (CDI I), and a toddler version, Words and Sentences, aimed at the age group 16 to $30 / 36$ months (CDI II). ${ }^{1}$

Both CDI I and CDI II have a strong focus on vocabulary, presenting checklists of words from different semantic domains and word classes for parents to tick off. For CDI I, a list of approximately 400 words can be ticked off for both comprehension and production, whereas in CDI II a list of approximately 700 words can be ticked off, for production only. In addition to the vocabulary checklists, CDI I contains checklists for gestures, play routines, and actions, whereas CDI II has additional checklists focusing on grammar (morphology, word combinations, and sentence complexity). Here, we will focus on the vocabulary part of the assessment tool.

The numbers of words mentioned above are based on the original American English CDI - in the different adaptations, the actual number of words may differ, but care is taken to make the vocabulary sets as comparable as possible through matching the semantic domains and word classes. An important point, as also stated by the MB-CDI advisory board (see https:// mb-cdi.stanford.edu/adaptations.html), is that an adaptation is not the same as a translation - and the adaptation must be not only linguistically 
but also culturally appropriate. To meet the criterion of comparability, central aspects of the CDI structure must be retained - however, the larger the linguistic and cultural distance between the American English original and the new language version, the more challenging the adaptation process.

Consider, for example, the differences between the adaptation to a language like Norwegian, a well-described language closely related to English, spoken in a context that is to a large extent culturally comparable to the original, and the adaptations to languages spoken in South Africa. For Norwegian, the adaptation process was relatively straightforward (Kristoffersen et al. 2013; Simonsen et al. 2014). A first Norwegian version, which closely followed the American original, was evaluated and modified by an expert group consisting of linguists and psychologists, resulting in (amongst other changes) more words in a few categories, based on linguistic differences. For instance, due to systematic differences in prepositions and location/direction terms between English and Norwegian, 15 words were added to the existing 26 words in this category. Some terms for family members were also added, namely two additional words for grandmother (bestemor) and two words for grandfather (bestefar), indicating whether these relatives are on the mother's (mormor/morfar) or the father's (farmor/farfar) side, as these terms are used frequently, alongside the generic bestemor/ bestefar. This version was piloted with a small group of 17 parents who, in addition to filling in the CDIs and a background questionnaire, were asked how long it took to fill them in, as well as to evaluate the instructions and variables, and to suggest possible new items to add. This resulted in a smallscale revision of the forms, e.g. adding words like $P C$ and pizza. Before the actual norming study, a second round of revision took place to make the Norwegian CDI more similar to the Danish version. The aim was to facilitate cross-linguistic comparisons of early language development in children acquiring Norwegian and Danish - two languages which are very similar in grammar and vocabulary, but markedly different in phonology.

This final version was then normed in a population-based study with approximately 6500 children, where parents completed the CDI forms over the internet - the first CDI study to use this methodology. Norway is a country with high rates of internet access, making this a feasible method - and it turned out to be very efficient, in terms of both time and coding accuracy. As has been found for many other CDI studies (Fenson et al. 2007), the sample of respondents was skewed in the direction of higher parental education (and thus higher SES), but the method of data collection did not seem to add to this skewness: For example, compared to the paper-based Danish CDI study (Bleses et al. 2008a), both the response rate and the skewness were very similar (Kristoffersen et al. 2013). The vocabulary in the final version was then validated against an existing corpus of Norwegian child language (Simonsen 1990) as well as through comparison with the words used in the three longest sentences reported by the parents in the CDI II form, and the validity was found to be acceptable (Kristoffersen et al. 2013). Later studies 


\section{Hanne Gram Simonsen and Frenette Southwood}

indicate that the parents' reports are valid down to the level of single items, as suggested by a strong correlation between age of acquisition based on the Norwegian CDI data and adults' ratings of subjective age of acquisition (Lind et al. 2015), strong correlation with frequency in child-directed speech (Hansen 2017), as well as good item-level agreement between children's responses in word recognition and parent reports on comprehension in the Norwegian CDI I (Lo et al. 2020).

In the South African context, where the languages at issue are spoken in cultural contexts far removed from the one for which the original American English CDI was developed, the adaptation was more complex for the multi-institutional, interdisciplinary, multilingual team involved therein ${ }^{2}$ (see Southwood et al. 2021). The American English CDI was translated by three mother-tongue speakers per language, and the collated translations were presented to language practitioners and to at least two focus groups (consisting of professional child service providers and parents of young children) in order to obtain advice on which words to omit and which words or synonyms to add. For instance, the words for matches were added, because many South African households make use of candles or paraffin lamps for light, and cooking food on an open fire (lit by matches or a lighter) is a common activity across cultures represented in South Africa. Words for moose and snowsuit, for example, which are not associated with South African life, were removed. Hereafter, 30-minute samples of naturally occurring language were collected from six toddlers per language, and words that occurred in the samples but not yet on the list were added.

The preliminary versions of the CDI I and II of each language were piloted with 40 caregivers of 8- to 18 -month-olds and 16-to 32-month-olds, respectively. For most languages, half of the children were situated in rural areas and the other half in urban or semi-urban areas. Typically, in South Africa, the rural/urban divide is also a lower/higher SES divide, so targeting across geographical locations enabled the inclusion of participants from a range of SES backgrounds. Only monolingual children were included in the pilot studies, but "monolingualism" was defined as receiving less than 4 hours per day of input in a language other than the L1, because including strictly monolingual children would have resulted in the pilot study participants not being representative of the general South African child population. Based on the results of this pilot, further words were removed or replaced before the second pilot study took place, this time with the caregivers of 100 infants and 100 toddlers per language, with the vast majority of these CDIs completed with the parent/caregivers by means of fieldworker interview (either face-toface or telephonically where COVID-19 restrictions necessitated the latter), following Alcock et al. (2015) who did CDI interviews in rural Kenya. This was necessary given the low literacy levels of many participating caregivers. The same protocol was followed simultaneously for a number of languages, but the final number of words varied across languages, because some words are polysemous in one language but require several related words 
in another. For example, porridge (a staple food for many South Africans) and cereal can both be called pap in Afrikaans, whereas porridge is referred to as amongst others makleu, motoho, mabele, or motoho wa mabele in Sesotho, and in Setswana, motogo refers to soft and bogobe to stiff sorghum porridge. Porridge made of maize meal is also common, referred to as papa and phaletšhe in Sesotho and Setswana, respectively. Kinship terms also differ amongst the languages. For instance, whereas English has one word for both male and female cousins, Afrikaans has two (niggie for a female and neef/nefie for a male cousin), with no collective term referring to either sex; and in Setswana, there are three words for sister (ausi "(big or small) sister," nkgonne "older sibling," and nnake "younger sibling," the latter two also used to refer to a brother). The number of semantic categories per language also varied across languages, because the CDIs for the two West Germanic languages contain pronouns and auxiliary and modal verbs, but the CDIs for the Bantu languages do not. ${ }^{3}$

As stated above, for Norwegian, there was a longitudinal child language corpus against which the Norwegian CDI could be validated. However, given the complete lack of child language corpora for most Southern African languages, the South African CDIs will be validated by means of language sample collection and language comprehension and production tasks, after which the validated CDIs will need to be normed. Given that multilingualism is the norm in South Africa, but also that the language combinations, and amount of exposure to, and proficiency in, each language vary widely even in the same community, the question arises as to what average length of daily exposure to languages other than the L1 should be targeted in the national norming study. Taking into account the sociocultural contexts in South Africa, the South African CDIs - unlike the Norwegian CDI - may need to be completed interview style during the norming study to enable representation of children whose caregivers have low levels of literacy and limited access to electronic devices and the internet.

Reliability and validity have been found to be good for the CDI tool (Fenson et al. 2007). However, it is worth remembering that due to variability in language development amongst young children, the tool should be used with care for the youngest age groups. Furthermore, for most of the norming studies, the samples are biased towards parents with higher education, resulting in those from lower SES being under-represented. During the development of the South African English CDIs, unlike the other South African CDIs, the sampling plan stipulated that half of the participants had to be from low SES backgrounds, regardless of their geographic location in terms of rural- or urban-situated. South African English is infrequently spoken as L1 in rural areas, but does vary according to SES (see Mesthrie 2002). For this reason, SES instead of geography was controlled for in the South African English sampling plan.

The fact that all CDIs are adapted from the same original has made them useful for cross-linguistic comparison, as exemplified in Bleses et al. (2008b), 
who compared CDIs across 17 different languages and found similar trends in vocabulary production across languages, in spite of large variation in children's language development. CDI-based cross-linguistic comparison has been made even more accessible through the Wordbank project (Frank et al. 2019; Braginsky et al. 2019).

For assessing multilingual children, the CDI has also been found to be a useful tool. For some contexts with extensive societal bilingualism, bilingual versions of the CDI have been developed; see, e.g. Maltese (Gatt 2007) and Irish (O'Toole and Fletcher 2010). In cases where such bi/multilingual versions do not exist, one could use a combination of monolingual versions of the CDI, provided that comparable versions indeed exist across the child's languages (see, e.g. Core et al. 2013 for Spanish-English; Gonzalez-Barrero, Schott, and Byers-Heinlein 2020 for French-English).

In a comparative study on bilingual vocabulary acquisition in children from six different language pairs, O'Toole et al. (2017) evaluated this latter method with CDI II (Words and Sentences), with the specific aim of identifying bilingual children at risk for language impairment. Two hundred and fifty typically developing children aged 2 to 3 years were included in the study. In addition to CDIs in the different languages, O'Toole et al. (2017) used a language background questionnaire to obtain information about risk factors for language impairment, language exposure, and demographic variables such as parental level of education and occupation. They found, as expected for bilingual children, higher scores for the L1 than the L2, and total vocabularies that were larger than conceptual vocabularies. They also found a wide variation in vocabulary scores both within and across the language pairs. This could be attributed to age, but also to the mother's education status, parental concern about language development, and amount of exposure to the $\mathrm{L} 2$.

Whereas O'Toole et al. (2017) conclude that comparing across language adaptations of the CDI identified potential milestones for multilingual development and also potential indicators of language delay, they point out several limitations. In accordance with common practice in CDI studies, they only used one person to report from each language. While there is an advantage to letting parents report on their children's language abilities, in particular if the language at issue is not known to the investigator or therapist, ideally two or three persons should report on each language, as recommended by De Houwer, Bornstein, and Leach (2005; see also De Houwer 2019). O'Toole et al. also found that parents sometimes misinterpreted the questions on language exposure - an effect also found in other studies (Quay 2008). In addition, as pointed out by Lanza (1997), what parents say about their language interaction with their children does not always correspond to what they actually $d o$. Thus, while language exposure patterns are indeed crucial for vocabulary acquisition, and need to be taken into account when seeking to establish multilingual norms (Gathercole, Thomas, and Hughes 2008), the complexity and variability in language interaction 
patterns across multilingual communities call for careful interpretation of such measures - a point to which we will return below.

Furthermore, O'Toole et al. (2017) found that direct comparison across languages was difficult due to the very large differences in the number of words between different CDI adaptations - this is a fact that has to be taken into account when calculating vocabulary sizes for analysis and comparison. Finally, the authors mention that, in addition to the differences in checklist sizes, typological differences between languages may play a role in vocabulary acquisition. As pointed out by Thordardóttir and Weismer (1996), languages with complex inflectional systems may make it more difficult for children to identify lexical entities from the input. The phonetic structure of the language may have the same effect (Bleses et al. 2008 b). Both inflectional systems and phonetic structure have been found to influence the acquisition of verbal morphology in Scandinavian children (Ragnarsdóttir, Simonsen, and Bleses 1998; Ragnarsdóttir, Simonsen, and Plunkett 1999), and it is likely that vocabulary acquisition will be affected in the same way, as suggested by Rescorla et al. (2017) in a comparison between Polish and English 2-year-olds. The above-mentioned factors - in addition to word frequencies in different languages and cultures - should be taken into account when evaluating vocabulary scores across languages in a multilingual child. This is especially important in contexts where limited research findings are available on language development norms. As will be expanded on further below, the extent to which local child-rearing practices include child-directed speech and the interrelatedness of sociocultural factors in the particular language community also need to be taken into consideration.

\section{Vocabulary Assessment through a Lexical Assessment Task - CLT}

Adaptation of parallel language versions of one single (monolingual) tool, as illustrated above in the case of the CDI, is one way to obtain a possible tool for multilingual language assessment. Whereas such adaptation of an existing tool is common (although less common than mere tool translation, which we strongly advise against), one can also construct a new multilingual assessment tool from scratch, which is what was done in the case of the LITMUS CLT.

CLT assesses production and comprehension of nouns and verbs through a picture-based test, targeting multilingual pre-school children (age range 3 to 6 years). Within the Bi-SLI COST network mentioned above, CLT was developed simultaneously for a multitude of languages, with target word selection being based on the same underlying criteria in order to obtain linguistic and cultural equivalence. Thus, each language version has its unique composition of target words with corresponding pictures, so that the words are not the same across languages, but they are meant to be equivalent across languages in terms of word complexity and age of acquisition. 
More language versions of CLT can also be added if the developers follow the established procedure for construction. Below follows a short overview of the construction procedure for CLT; a thorough description thereof and the rationale behind CLT can be found in Haman, tuniewska, and Pomiechowska (2015). A selection of studies carried out with CLT in different languages can be found in Potgieter and Southwood (2016) and in a special issue of Clinical Linguistics and Phonetics (2017).

Given that CLT is a picture-based task, the first step was for 1000 pictures (depicting objects and actions) from various sources to be named and evaluated for linguistic and cultural appropriateness by competent adult judges across 34 languages. The result was a set of 299 pictures that each evoked a single word with the same English translation in a reliable way in each of the languages, and these pictures were then redrawn in a unified style and rated for cultural equivalence. In the next step, the words associated with the 299 pictures were rated for subjective age of acquisition: At least 20 raters in each language were asked how old they thought they were when they acquired each of these words (see Euniewska et al. 2016 for a description of this study). Finally, a composite complexity index was created for each word, based on phonological, morphological, and etymological features, as well as children's exposure to that word, rated by competent L1 judges of each language (see Haman et al. 2015, for details about the complexity index). Based on age of acquisition and complexity index values for the words in each language, four tasks were constructed, assessing production and comprehension of nouns and verbs, respectively, with 32 target words in each task. In the production tasks, the child is shown one picture per page and is requested to name the depicted object or action. In the comprehension tasks, the child is shown four pictures per page (one for the target word and three distractors with similar complexity indexes and ages of acquisition) and is asked to choose the picture that corresponds to the target object or action.

Although the CLT has not yet been normed for any language or language combination, several studies have shown its usefulness for different purposes, such as measuring the language proficiency of multilinguals (e.g. Altman, Goldstein, and Armon-Lotem 2017; Van Wonderen and Unsworth 2020), investigating the interaction between vocabulary size and other factors in multilinguals (Hansen et al. 2017; Altman, Goldstein, and ArmonLotem 2018), comparing different assessment tools for multilingual children (Abbot-Smith et al. 2018; Hansen et al. 2019); determining the comparative vocabulary size in the languages of young typically developing multilingual children (e.g. Potgieter and Southwood 2016; Lindgren and Bohnacker 2020); and differentiating between children (multilingual or monolingual) with and without language impairment (Khoury Auoad Saliby et al. 2017; Kapalková and Slančová 2017).

To investigate the comparability of the CLTs across languages, Haman et al. (2017) conducted a large-scale comparison between monolingual 
children's performance on the CLT across 17 languages from eight different language families: Baltic (Lithuanian); Bantu (isiXhosa); Finnic (Finnish); Germanic (Afrikaans, British English, South African English, German, Luxembourgish, Norwegian, Swedish); Romance (Catalan, Italian); Semitic (Hebrew); Slavic (Polish, Serbian, Slovak); and Turkic (Turkish). Six hundred and thirty-nine typically developing children were included, with an age range of 3 to 6 years. SES data were available for participants from 11 of the languages; most of them came from a mid-to-high SES background. The exception was the participants from South Africa (speaking Afrikaans, isiXhosa, and/or South African English), where detailed SES information was collected as part of another study (Potgieter and Southwood 2016). Here, half of the participants speaking Afrikaans and South African English, and all the participants speaking isiXhosa, had a low SES background. For the six languages where no SES data were available, recruitment information suggested a mid-to-high SES environment.

Overall results showed similar trends across the languages: A stable word class effect (nouns easier than verbs) and language mode effect (comprehension easier than production), as well as a general age effect (vocabulary size growth with age), indicating cross-linguistic comparability. However, concerning vocabulary size, while there were only small differences between 16 of the languages, isiXhosa-speaking children obtained significantly lower scores than participants from the other languages. The reason for this result is not clear. It may be due to the small sample size (10) of the isiXhosaspeaking children and/or because they were among the younger participants in the study. However, that may not be the full answer.

A first hypothesis could be that isiXhosa-speaking pre-schoolers indeed have small vocabularies compared to those of the child speakers of the other languages included in the study, possibly because of their low SES. Note that when comparing the vocabulary sizes as measured by the CDI in the data of Southwood et al. (2021), isiXhosa-speaking toddlers are shown to have statistically significantly smaller total vocabularies than toddlers who speak Afrikaans, South African English, or Xitsonga. This finding requires further investigation because it is not clear why isiXhosa-speaking children specifically would have smaller vocabularies than, for instance, Xitsonga children who grow up in comparable ecological settings and in households with comparable SES. There could be sociocultural reasons that influence parent reporting in the two isiXhosa-speaking communities that took part in the second South African CDI pilot study. It could also be that language socialisation practices in isiXhosa-speaking communities do not offer opportunities for rapid vocabulary acquisition early on in a child's life, but that these change over time (as the child becomes a more proficient conversational partner), allowing isiXhosa-speaking pre-schoolers to catch up before school entry with their peers who have other home languages. For instance, among the Black African population in South Africa (which includes almost all home language speakers of isiXhosa), two-thirds of children aged 0 to 
6 years rarely or never receive an explanation from their household members when they point to objects and ask for an explanation (Statistics South Africa 2018a). Whereas the frequent absence of such explanations in the Black African population cannot account for the difference in vocabulary size between the isiXhosa and Xitsonga CDI data sets, it does show that more careful investigation of language socialisation practices amongst smaller age bands of children is needed, preferably obtained by means of cross-sectional studies with large participant numbers, or longitudinal studies.

A second hypothesis for why the isiXhosa-speaking pre-schoolers had low CLT scores could pertain to the task, namely that the type of adult-child interaction around pictures was culturally unfamiliar to the pre-schoolers. Indeed, $48 \%$ of South African children never read a book with a parent or guardian (Statistics South Africa 2018a), resulting in infrequent adult-child engagement around meaningful pictures. In fact, South African households typically have very few books, with $58 \%$ of South Africans over the age of 15 living in households in which there are no books (South African Book Development Council 2016). Furthermore, naming appears not to be modelled frequently: According to Statistics South Africa (2018b), 54\% of South African parents do not name objects while interacting with their children of 3 years and younger. The reason for the comparatively small isiXhosa vocabulary as assessed by the CLT could have to do with the ecological validity of the task for the pre-schoolers rather than with linguistic limitations on the part of the isiXhosa-speaking pre-schoolers. Interestingly, subjective age of acquisition for a set of 299 words (as reported by adult speakers) was found to be significantly later for isiXhosa than for 24 other languages (Euniewska et al. 2016). This, together with the finding of a smaller vocabulary of isiXhosa-speaking pre-schoolers, warrants further investigation. It could be that cross-linguistic comparison with other, still to be studied South African languages would yield some insights in this regard.

\section{Insights and Challenges}

Our work on child language assessment across different multilingual contexts, and specifically in the construction of assessment tools with different language versions, has given us some important insights. While we acknowledge the importance of and necessity for linguistically and culturally comparable assessment tools for cross-linguistic comparison (within and across language families), it is not the case that as long as an assessment tool has comparable language versions, these can necessarily be used with any multilingual child to indicate linguistic strengths and weaknesses accurately (see also Van Wonderen and Unsworth 2020). As stated above, one of the purposes of multilingual language assessment is to identify children with language delay or language impairment. Whereas linguistically comparable assessment tools go a long way towards enabling such identification of language delay or impairment in multilingual children, our experiences 
in various contexts have shown that linguistic comparability alone is insufficient - one also needs, at least, norms for the multilingual population at issue as well as a thorough knowledge of local language socialisation practices.

As shown in the case of isiXhosa above, it could be that one language population has a smaller vocabulary size than others at a particular age. Without language development norms, a child might well be misdiagnosed with a language delay or language impairment despite possessing typical language skills for his/her age according to his/her language community. Doing so in the name of linguistically comparable language versions would render an incorrect representation of the child's linguistic skills, and instruments that identify the majority of child members of a multilingual community as presenting with a language delay or impairment are not useful, regardless of how linguistically comparable these tools are.

Knowledge exists on language socialisation as practised in the North, but our knowledge of how children are socialised linguistically in the South is still very limited. That naming of objects when interacting with children is not common in South Africa is significant for developers of child language assessment tools, as many of these tools are based on naming pictures or objects, and requiring a child to name objects when such naming is not frequently modelled to him/her may directly affect the child's language test score, regardless of the level of linguistic comparability between different language versions of the tool. Also, the fact that about half of South African children are not used to joint book-reading with adults could place them at a disadvantage in terms of required CLT responses compared to children who are used to looking at pictures with adults, pointing to and discussing such pictures, or answering questions about them. Indeed, not only the content of an instrument but also the manner in which it is administered can affect children's test scores, and it is not in all instances possible to ensure that culturally comparable administration methods are used.

Our work has convinced us that tool adaptation without sufficient regard for local contexts cannot lead to valid assessment tools. A lot of research has been done (almost exclusively in the North) on the influence of SES and the quality, quantity, and age of onset of language exposure on monolingual and multilingual children's language development. This is of course both necessary and important, but more than knowledge of SES and language exposure is needed in order to develop valid and reliable assessment tools and to interpret assessment results; we also need insight into the different manners in which children are exposed to and interact in their languages, both within and outside their homes. This means taking language socialisation practices in the community into account - as pointed out more than 30 years ago by Ochs and Schieffelin: both "socialisation through the use of language and socialisation to use language" (Schieffelin and Ochs 1986, 163). In a Northern context, Lanza (1997) took this perspective in her groundbreaking analysis of language mixing in infant multilingualism. 


\section{Hanne Gram Simonsen and Frenette Southwood}

From the early literature on child-directed speech (Snow and Ferguson 1977; Gleitman, Newport, and Gleitman 1984), we can recognise characteristic patterns for adults speaking to children in a Western middle-class culture: Both in form and content, adults adapt their language to the child's level and situation, and often they "give" the child the words through naming the objects around them. However, such patterns of child-centred adaptation are not universal, but culture-dependent, as has been shown early on across different cultures. Language communities that do not show this pattern include Luo in Kenya (Blount 1971); Kaluli in Papua New Guinea (Ochs and Schieffelin 1982); an African-American working-class community in rural Carolina, USA (Heath 1983); Inuit communities in Canada (Crago, Annahatak, and Ningiuruvik 1993); and Western Samoa (Ochs 1984, 1988; Simonsen 1990). More recently, similar findings have been documented in a village in the north-east of Botswana (Geiger and Alant 2005) and for Tsimane in Bolivia (Cristia et al. 2019). The reasons for not using such child-centred adaptation may vary across cultures. For example, in Botswana, the mothers reported not conversing with young children because the children could not speak yet and were therefore not considered conversation partners. In Western Samoa, a strictly hierarchical society, this was part of the reason for not conversing frequently with young children, but children also had a low social status in the community, and cultural norms entailed that those of higher social status do not assist those of lower status. Engaging in child-centred speech would entail such assistance, and therefore children were required to express themselves clearly, without adult assistance, before adults engaged with them in conversation. This way, the Samoan children were socialised into the hierarchical structure of their society, linguistically and otherwise (Ochs 1988; Simonsen 1990).

However, even though differences in child-centredness have been reported, it is not the case that all non-WEIRD communities are similar. For instance, Rabain-Jamin (1998) found that Wolof-speaking mothers' speech to infants and toddlers in rural Senegal contained frequent prompting and reported speech, and mothers also adapted their speech to the linguistic maturity of their child. The prompting reportedly occurred because mothers felt it necessary to verify that their toddler's expressive language capacity was intact - in Wolof culture, remaining quiet for too long could be socially unacceptable, and mothers do not want their child to be labelled a person who withdraws from social exchange. According to Rabain-Jamin (1998), both prompts and reported speech in polyadic contexts relate to a system of speech mediations and information exchange and serve to assist the child to internalise the (complex) cultural system of social regulation. Likewise, Demuth (1986) found that Sesotho-speaking children are frequently taught linguistic and social routines, and that adults differed in their views on how useful verbal interactions with children are. One view was that speaking to infants is helpful, and that learning to speak well was a valued part of learning the Sesotho language. These examples show that not only can findings 
from WEIRD contexts not be applied directly to non-WEIRD contexts, but there is no such thing as a universal non-WEIRD context as regards language socialisation. (And there is probably no universal WEIRD context either.)

\section{Conclusion}

Comparable language versions of a tool are indeed needed for use with multilingual children (in the absence of multilingual tools, that is), and much progress has been made in this regard. Linguistic and cultural adaptations of language assessment tools for use with monolingual and multilingual children now exist, and some adaptations do take into account the influence of SES and language exposure patterns. However, we are not aware of any child language assessment tools that take into account language socialisation practices in different language communities. This could be one of the reasons for comparable language versions not always rendering directly comparable results. We do not yet have answers to the question as to how one should assess language socialisation practices, nor to how one would integrate knowledge on language socialisation practices with knowledge gained through the use of assessment tools, whether those used in direct assessment such as CLT or those that are parents' reports such as CDIs. However, a first step towards finding such answers would be to acknowledge that fair and valid assessment tools can hardly be developed without taking them into account.

There is a long and productive tradition of child language research in the North. The South can learn lessons from the North and should increase the extent to which it generates its own research findings on the assessment of language skills in its multilingual children. However, there might also be lessons for the North to learn from the South where the variability and complexity of childhood multilingualism highlight the need for mindfulness when constructing, using and interpreting child language assessment tools.

\section{Acknowledgements}

The authors thank Pernille Hansen and Michelle White for their helpful comments on an earlier version of this chapter, as well as two anonymous reviewers for useful suggestions. We also acknowledge financial support from the following sources without which this collaboration would not have been possible: The European Union Cooperation for Science and Technology (COST Action IS0804, Bi-SLI); The National Research Foundation of South Africa (HSD170602236563); The Norwegian Research Council through its Centres of Excellence Scheme, Project number 223265; INTPART (International Partnership for Excellent Education and Research) and the South African Centre for Digital Language Resources. 


\section{Hanne Gram Simonsen and Frenette Southwood}

Any opinion, findings, conclusions or recommendations expressed in this material are those of the authors.

\section{Notes}

1 For some languages, a CDI III has also been developed, aimed at children above 36 months, but it is less widely adapted and used, and will not be discussed here.

2 The team consists of Frenette Southwood, Helena Oosthuizen, and Nina Brink for Afrikaans; Tessa Dowling, Emma Whitelaw, Martin Mössmer, and Sefela Yalala for isiXhosa; Michelle Pascoe and Olebeng Mahura for Setswana; Heather Brookes and Sefela Yalala for Sesotho; Frenette Southwood, Helena Oosthuizen, and Michelle White for South African English; Mikateko Ndhambi for Xitsonga; and Katie Alcock as consultant based on the work on Kiswahili and Kigiriama versions of the CDI in Kenya. Collaborators, also for other language versions, are being added to the team as they are identified.

3 Pronouns do occur in the Bantu languages, but they are used, for instance, for emphatic statements, and their construction is very varied as they also use agreement. Pronouns did not form a part of the vocabulary of the children in our pilot studies who spoke Bantu languages, and were thus not included in later versions of the CDI in those languages.

\section{References}

Abbot-Smith, K., P. Morawska-Patera, M. Łuniewska, M. Spruce, and E. Haman. 2018. "Using Parental Questionnaires to Investigate the Heritage Language Proficiency of Bilingual Children." Child Language Teaching and Therapy 34 (2): $155-170$.

Alcock, K., K. Rimba, P. Holding, P. Kitsao-Wekulo, A. Abubakar, and C.R.J.C. Newton. 2015. "Developmental Inventories Using Illiterate Parents as Informants: Communicative Development Inventory (CDI) Adaptation for Two Kenyan Languages." Journal of Child Language 42: 763-785.

Altman, C., T. Goldstein, and S. Armon-Lotem. 2017. "Quantitative and Qualitative Differences in the Lexical Knowledge of Monolingual and Bilingual Children on the LITMUS-CLT task." Clinical Linguistics and Phonetics 31 (11-12): 931-954.

Altman, C., T. Goldstein, and S. Armon-Lotem. 2018. "Vocabulary, Metalinguistic Awareness and Language Dominance Among Bilingual Preschool Children." Frontiers in Psychology 9: 1953.

Armon-Lotem, S., J. de Jong, and N. Meir, eds. 2015. Assessing Multilingual Children: Disentangling Bilingualism from Language Impairment. Bristol: Multilingual Matters.

Bedore, L.M. and E.D. Peña. 2008. "Assessment of Bilingual Children for Identification of Language Impairment: Current Findings and Implications for Practice." International Journal of Bilingual Education and Bilingualism 11: $1-29$.

Bleses, D., W. Vach, M. Slott, S. Wehberg, P. Thomsen, T.O. Madsen, and H. Basbøll. 2008a. "The Danish Communicative Developmental Inventories: Validity and Main Developmental Trends." Journal of Child Language 35 (3): 651-669. 
Bleses, D., W. Vach, M. Slott, S. Wehberg, P. Thomsen, T. O. Madsen, and H. Basbøll. 2008b. "Early Vocabulary Development in Danish and Other Languages: A CDI-based Comparison.” Journal of Child Language 35 (3): 619-650.

Blount, B.G. 1971. "Socialization and Pre-Linguistic Development Among the Luo of Kenya." Southwestern Journal of Anthropology 27 (1): 41-50.

Braginsky, M., D. Yurovsky, V.A. Marchman, and M.C. Frank. 2019. "Consistency and Variability in Children's Word Learning Across Languages.” Open Mind 3: 52-67.

Butler, Y.G. 2013. "Bilingualism/Multilingualism and Second-Language Acquisition." In The Handbook of Bilingualism and Multilingualism, 2nd edition, edited by T.K. Bhatia and W.C. Ritchie, 109-136. Oxford: Wiley-Blackwell.

Core, C., E. Hoff, R. Rumiche, and M. Señor. 2013. "Total and Conceptual Vocabulary in Spanish-English Bilinguals from 22 to 30 Months: Implications for Assessment." Journal of Speech, Language, and Hearing Research 56 (5): 1637-1649.

Crago, M., B. Annahatak, and L. Ningiuruvik. 1993. "Changing Patterns of Language Socialization in Inuit Homes." Anthropology and Education Quarterly 24 (3): 205-223.

Cristia, A., E. Dupoux, M. Gurven, and J. Stieglitz. 2019. "Child-directed Speech is Infrequent in a Forager-farmer Population: A Time Allocation Study." Child Development 90 (3): 759-773.

De Houwer, A. 2019. "Equitable Evaluation of Bilingual Children's Language Knowledge Using the CDI: It Really Matters Who You Ask." Journal of Monolingual and Bilingual Speech 1 (1): 32-54.

De Houwer, A., M.H. Bornstein, and D.B. Leach. 2005. "Assessing Early Communicative Ability: A Cross-reporter Cumulative Score for the MacArthur CDI." Journal of Child Language 32 (4): 735-758.

Demuth, K. 1986. "Prompting Routines in the Language Socialization of Basotho Children." In Language Socialization Across Cultures, edited by B. Schieffelin and E. Ochs, 51-79. Cambridge: Cambridge University Press.

Fenson, L., V.A. Marchman, D.J. Thal, P.S. Dale, J.S. Reznick, and E. Bates. 2007. MacArthur-Bates Communicative Development Inventories. User's Guide and Technical Manual. Baltimore: Brookes.

Frank, M.C., M. Braginsky, D. Yurovsky, and V.A. Marchman. 2019. Variability and Consistency in Early Language Learning: The Wordbank Project. Cambridge, MA.: MIT Press.

Gathercole, V.C.M., E.M. Thomas, and E. Hughes. 2008. "Designing a Normed Receptive Vocabulary Test for Bilingual Populations: A Model from Welsh." International Journal of Bilingual Education and Bilingualism 11 (6): 678-720.

Gatt, D. 2007. "Establishing the Concurrent Validity of a Vocabulary Checklist for Young Maltese Children.” Folia Phoniatrica et Logopaedica 59 (6): 297-305.

Geiger, M. and E. Alant. 2005. "Child-rearing Practices And Children's Communicative Interactions in a Village in Botswana.” Early Years 25: 183-191.

Gleitman, L.R., E.L. Newport, and H. Gleitman. 1984. "The Current Status of the Motherese Hypothesis." Journal of Child Language 11 (1): 43-79.

Gonzalez-Barrero, A.M., E. Schott, and K. Byers-Heinlein. 2020. "Bilingual Adjusted Vocabulary: A Developmentally-informed Bilingual Vocabulary Measure.” PsyArXiv, September 3. doi:10.31234/osf.io/x7s4u 


\section{Hanne Gram Simonsen and Frenette Southwood}

Haman, E., M. Łuniewska, and B. Pomiechowska. 2015. "Designing Crosslinguistic Lexical Tasks (CLTs) for Bilingual Preschool Children.” In Assessing Multilingual Children: Disentangling Bilingualism from Language Impairment, edited by S. Armon-Lotem, J. de Jong, and N. Meir, 196-240. Bristol, UK: Multilingual Matters.

Haman, E., M. Łuniewska, P. Hansen, H. G. Simonsen, S. Chiat, J. Bjekić, A. Blažiene, et al. 2017. "Noun and Verb Knowledge in Monolingual Preschool Children Across 17 Languages: Data from Cross-Linguistic Lexical Tasks (LITMUS-CLT)." Clinical Linguistics and Phonetics 31 (11-12): 818-843.

Hansen, P. 2017. "What Makes A Word Easy to Acquire? The Effects of Word Class, Frequency, Imageability and Phonological Neighbourhood Density on Lexical Development.” First Language 37 (2): 205-225.

Hansen, P., H.G. Simonsen, M. Łuniewska, and E. Haman. 2017. "Validating the Psycholinguistic Aspects of LITMUS-CLT: Evidence from Polish and Norwegian." Clinical Linguistics and Phonetics 31 (11-12): 910-930.

Hansen, P., M. Łuniewska, H.G. Simonsen, E. Haman, K. Mieszkowska, J. Kołak, and Z. Wodniecka. 2019. "Picture-based Vocabulary Assessment versus Parental Questionnaires: A Cross-Linguistic Study of Bilingual Assessment Methods." International Journal of Bilingualism 23 (2): 437-456.

Heath, S.B. 1983. Ways with Words: Language, Life and Work in Communities and Classrooms. Cambridge: Cambridge University Press.

Henrich, J., S. Heine, and A. Norenzayan. 2010. "Most People are not WEIRD." Nature 466: 29.

Kapalková, S. and D. Slančová, 2017. “The Vocabulary Profile of Slovak Children with Primary Language Impairment Compared to Typically Developing Slovak Children Measured by LITMUS-CLT." Clinical Linguistics \& Phonetics 31(1112): 893-909.

Khoury Aouad Saliby, C., C. Dos Santos, E. Kouba Hreich, and C. Messarra. 2017. "Assessing Lebanese Bilingual Children: The Use of Cross-Linguistic Lexical Tasks in Lebanese Arabic." Clinical Linguistics and Phonetics 31 (11-12): 874-892.

Kristoffersen, K.E., D. Bleses, S. Wehberg, R.N. Jørgensen, E.A. Eiesland, L.Y. Henriksen, and H.G. Simonsen. 2013. "The Use of the Internet in Collecting CDI Data - An Example from Norway." Journal of Child Language 40 (3): 567-585.

Lanza, E. 1997. Language Mixing in Infant Bilingualism: A Sociolinguistic Perspective. Oxford: Oxford University Press.

Lanza, E. 2007. "Multilingualism and the Family." In Handbook of Multilingualism and Multilingual Communication, edited by P. Auer and L. Wei, 45-68. Berlin; Boston: De Gruyter Mouton.

Lind, M., H.G. Simonsen, P. Hansen, E. Holm, and B.H. Mevik. 2015. "Norwegian Words: A Lexical Database for Clinicians and Researchers." Clinical Linguistics and Phonetics 29 (4): 276-290.

Lindgren, J. and U. Bohnacker. 2020. "Vocabulary Development in Closely-related Languages: Age, Word Type and Cognate Facilitation Effects in Bilingual Swedish-German Preschool Children." Linguistic Approaches to Bilingualism 10 (5): 587-622.

Lo, C.H., A. Rosslund, J.H. Chai, J. Mayor, and N. Kartushina. 2020. “Tablet Assessment of Word Comprehension Reveals Coarse Word Representations in 18-20-Month-old Toddlers." PsyArXiv, October 31. doi:10.31234/osf.io/7hpgm 
Łuniewska, M., E. Haman, S. Armon-Lotem, B. Etenkowski, F. Southwood, D. Anđelković, E. Blom, et al. 2016. "Ratings of Age of Acquisition of 299 Words Across 25 Languages: Is There a Cross-linguistic Order of Words?” Behavior Research Methods 48 (3): 1154-1177.

Mesthrie, R., ed. 2002. Language in South Africa. Cambridge, UK: Cambridge University Press.

Ochs, E. 1984. "Clarification and Culture." In Meaning, Form and Use in Context: Linguistic Applications, edited by D. Schiffrin, 325-341. Georgetown: Georgetown University Round Table.

Ochs, E. 1988. Culture and Language Development. Language Acquisition and Language Socialization in a Samoan Village. Cambridge: Cambridge University Press.

Ochs, E. and B. Schieffelin. 1982. "Language Acquisition and Socialization: Three Developmental Stories and their Implications." Sociolinguistic Working Papers no. 105. Austin: Southwest Educational Development Laboratory.

O’Toole, C. and P. Fletcher. 2010. "Validity of a Parent Report Instrument for IrishSpeaking Toddlers." First Language 30(2): 199-217.

O’Toole, C., D. Gatt, T.M. Hickey, A. Miękisz, E. Haman, S. Armon-Lotem, T. Rinker, O. Ohana, C. dos Santos, and S. Kern. 2017. "Parent Report of Early Lexical Production in Bilingual Children: A Cross-Linguistic CDI Comparison.” International Journal of Bilingual Education and Bilingualism 20 (2): 124-145.

Potgieter, A.P. and F. Southwood. 2016. "A Comparison of Proficiency Levels in 4-year-old Monolingual and Trilingual Speakers of Afrikaans, isiXhosa and South African English Across SES Boundaries, Using LITMUS-CLT.” Clinical Linguistics and Phonetics 30 (2): 87-100.

Quay, S. 2008. "Dinner Conversations with a Trilingual Two-year-old: Language Socialization in a Multilingual Context." First Language 28 (1): 5-33.

Rabain-Jamin, J. 1998. "Polyadic Language Socialization Strategy: The Case of Toddlers in Senegal." Discourse Processes 26 (1): 43-65.

Ragnarsdóttir, H., H.G. Simonsen, and D. Bleses. 1998. "Experimental Evidence on the Acquisition of Past Tense Inflection in Danish, Icelandic and Norwegian Children." In Papers in First Language Acquisition, edited by D. Bleses and J. Wagner, 23-42. Working Papers in Language and Communication, 16. Odense, Denmark: Odense Universitets Trykkeri.

Ragnarsdóttir, H., H.G. Simonsen, and K. Plunkett. 1999. "The Acquisition of Past Tense Morphology in Icelandic and Norwegian Children: An Experimental Study." Journal of Child Language 26 (3): 577-618.

Rescorla, L., H. Constants, M. Białecka-Pikul, M. Stępień-Nycz, and A. Ochał. 2017. "Polish Vocabulary Development in 2-year-olds: Comparisons with English using the Language Development Survey." Journal of Speech, Language, and Hearing Research 60 (4): 1029-1035.

Schieffelin, B.B., and E. Ochs. 1986. "Language Socialization." Annual Review of Anthropology 15 (1): 163-191.

Simonsen, H.G. 1990. Barns Fonologi: system og variasjon hos tre norske og et samoisk barn. [Children's Phonology: System and Variation in Three Norwegian Children and One Samoan Child]. Unpublished doctoral dissertation, University of Oslo.

Simonsen, H.G., K.E. Kristoffersen, D. Bleses, S. Wehberg, and R.N. Jørgensen. 2014. "The Norwegian Communicative Development Inventories: Reliability, 


\section{Hanne Gram Simonsen and Frenette Southwood}

Main Developmental Trends and Gender Differences.” First Language 34 (1): 3-23.

Snow, C.E. and C.A. Ferguson. 1977. Talking to Children. Cambridge: Cambridge University Press.

South African Book Development Council. 2016. National Survey into the Reading and Book Reading Behaviour of Adult South Africans. Accessed November 21, 2020. https://sabookcouncil.co.za/wp-content/uploads/2019/08/Final-ReportNRS-2016.pdf

Southwood, F., M.J. White, H. Brookes, M. Pascoe, M. Ndhambi, S. Yalala, O. Mahura, et al. 2021. "Sociocultural Factors Affecting Vocabulary Development in Young South African Children." Frontiers in Educational Psychology .12, 1645. https://doi.org/10.3389/fpsyg.2021.642315

Statistics Norway. 2020a. "Kindergartens: 12272: Children from Linguistic and Cultural Minorities 2015 - 2019.” Accessed December 1, 2020. https://www.ssb. no/en/statbank/table/12272/

Statistics Norway. 2020b. Accessed December 1, 2020. https://www.ssb.no/en/utd anning/statistikker/barnehager

Statistics South Africa. 2018a. Education Series Volume IV: Early Childhood Development in South Africa, 2016. Pretoria, South Africa: Statistics South Africa.

Statistics South Africa. 2018b. 2017 General Household Survey. Pretoria, South Africa: Statistics South Africa.

Statistics South Africa. 2019. "Five Facts about Poverty in South Africa." Accessed April 5, 2020. http://www.statssa.gov.za/?p=12075

Thordardóttir, E.T. and S.E. Weismer. 1996. "Language Assessment via Parent Report: Development of a Screening Instrument for Icelandic Children.” First Language 16(48): 265-285.

Van Wonderen, E. and S. Unsworth. 2020. "Testing the Validity of the CrossLinguistic Lexical Task as a Measure of Language Proficiency in Bilingual Children." Journal of Child Language, Advance online publication: 1-25. doi:10.1017/S030500092000063X.

Wei, L. 2013. "Conceptual and Methodological Issues in Bilingualism and Multilingualism Research." In The Handbook of Bilingualism and Multilingualism, 2nd edition, edited by T.K. Bhatia and W. Ritchie, 26-41. Oxford: Wiley-Blackwell. 


\title{
2 Developmental Perspectives on Parents' Use of Discourse Strategies with Bilingual Children
}

\author{
Annick De Houwer and Janice Nakamura
}

\section{Introduction}

In likely the first systematic study of language choice by a bilingual child, De Houwer (1983) found that already between the ages of 2;7 (years;months) and 3;4, Dutch-English Kate adjusted her language choice according to whom she was addressing. ${ }^{1}$ Kate spoke English to her mother (whose Dutch proficiency was minimal), Dutch to her monolingual Dutch-speaking grandparents, and mostly Dutch but also some English to her father and the investigator. Both Kate's father and the investigator addressed Kate in Dutch but also spoke fluent English, as the child had often overheard. On the few occasions (38 in a corpus of over 6000 exchanges collected over nine months) that the adults did not speak the language they normally used with Kate, she usually replied in the same 'unusual' language, thus following the Convergent Choice Principle or CCP (De Houwer 2019).

Indeed, following the CCP is the default. Bilinguals tend to speak the same way as their interlocutors, either speaking the same single language or using a mixture of the same languages, depending on what pattern has been established in their interaction history. The marked case occurs when bilinguals sharing the same set of languages consistently speak a different language to each other (De Houwer 2019), in what we term dual-lingual conversations.

By age 3, Kate addressed people she mostly heard speaking just a single language in that same language. This makes sense, given that even very young children want to make sure they are understood. However, with people she knew to be fluent speakers of the same two languages she spoke, Kate would use either language. She did not have to monitor so much which language she spoke (De Houwer 1983): She could assume bilingual speakers would understand her. Thus, it was unnecessary to stick to just one language. However, Kate still addressed her bilingual interlocutors mostly in Dutch, the language they mostly spoke with her.

How did Kate learn to do so? She was likely socialized into this pattern of response when she was younger. Elizabeth Lanza was the first to present a comprehensive analysis systematically addressing socialization patterns 
regarding language choice in conversations between a bilingual parent and a bilingually raised young child. Henceforth, we refer to this analysis as Lanza's "bilingual family interaction model", BIFIM for short and explained below (in an email on January 7, 2015, Lanza gave De Houwer the permission to use this term in reference to her work).

The first author first learned about Lanza's pioneering work in (1987) at her conference presentation on "Code-Mixing and Code-Switching in the Bilingual Infant's Discourse", which proposed a first partial version of BIFIM. Lanza (1988) was the first publication relating to the model. It focused on interactions between Norwegian-English Siri at 2;2 and her bilingual parents. Lanza (1992) was based on more extensive data from Siri between ages $2 ; 0$ and $2 ; 7$, and included the first comprehensive publication regarding BIFIM. More detailed analyses of the Siri data formed the bulk of the empirical study in Lanza's dissertation (1990), which additionally discussed data from Norwegian-English Tomas, who was studied for nearly four months after his second birthday. Lanza's dissertation was the basis for her book (1997b; reprinted in 2004 with slight modifications and a new Afterword). Lanza (1997a) and Lanza (2001) also discussed data for Siri and/or Tomas. Both children's families were middle class and consisted of a Norwegian father and an American mother. They lived in Oslo, Norway. All four parents were Norwegian-English bilinguals.

BIFIM was developed for children who acquire two languages (Language Alpha and Language A) simultaneously in the home from birth (Bilingual First Language Acquisition or BFLA). BFLA children may use unilingual utterances in either Language Alpha or Language A. They may also use mixed utterances, which combine morphological elements from both languages (De Houwer 2009, 2019). BIFIM was proposed to describe how parents respond to BFLA children's language mixing, defined as their use of two languages within a conversation or utterance (Lanza 1997b, 3). It thus describes how, within a single conversation, parents who speak Language A respond to children speaking Language Alpha or using a mixed utterance.

Figure 2.1 presents BIFIM. As in De Houwer (2009), it has been slightly adapted from Lanza (1997b, 261-269). Lanza's term “code-switching” was changed to "Language-Switching" since "code-switching" can have different meanings depending on particular theoretical perspectives, a problem Lanza also acknowledges.

The top of Figure 2.1 shows that parents speaking Language A to children can ask for clarification if children used Language Alpha instead of Language A (Minimal Grasp Strategy, MGS). This may encourage children to switch to Language A (children need not interpret such clarification requests this way; see Lanza 1997b, 305). Below we treat translation requests as in What does Shelly say? (Chevalier 2015) as instances of MGS. Next, children may also be asked to confirm the Language A alternative offered by parents (Expressed Guess Strategy, EGS). MGS and EGS encourage children to speak the same language as their parents. Thus, they are 
What do parents who normally speak Language A to children do when children speak Language Alpha to them or use a mixed utterance?

\section{Most monolingual discourse strategies}

\section{Minimal Grasp Strategy (MGS)}

Parents use Language A to ask children to clarify their utterance (as in: 'what did you say?' or 'I don't know what you mean'). The expectation is that children will respond to such requests for clarification by changing to Language $\mathrm{A}$.

\section{Expressed Guess Strategy (EGS)}

Parents use a question in Language A that translates what they thought children intended to say. This constitutes a direct or indirect request for confirmation and takes the form of a yes-no-question (as in: 'did you mean to say you want something to drink?', or 'a drink?'). The expectation is that children will respond to the request in Language A.

\section{Adult Repetition (AR)}

Parents repeat the child utterance in Language A. There is no request for children to respond, although some children may in turn repeat the parental repetition in Language A.

\section{Move On Strategy (MOS)}

Parents do not use any of the three preceding strategies but just continue talking in Language A

\section{$\checkmark$ Language Switching (LS)}

Parents switch to Language Alpha or a mixed utterance and do not stick to Language A. Most bilingual discourse strategies

\section{Figure 2.1 Lanza's (1992, 1997b) bilingual family interaction model (BIFIM)}

monolingual discourse strategies. Simply repeating in Language A what children said in Language Alpha (Adult Repetition, AR) can be taken as (1) a correction of child language choice (a monolingual strategy), (2) a confirmation of the contents of an utterance (neutral as to language choice), or (3) a teaching strategy showing children what their utterance translates as (also neutral). Because of its ambiguity, AR is neither a monolingual nor a bilingual strategy (but see Section 2 when we discuss children between ages 2 and 4). Bilingual strategies consist of parents just continuing to use Language A without paying attention to children's use of Language Alpha (Move On Strategy, MOS), and, indeed, of parents changing to Language Alpha themselves (Language-Switching, LS). As Lanza (1997b, 261) recognizes, BIFIM forms only part of the totality of discourse strategies parents use in interaction with their children.

BIFIM is not just a descriptive model. It also helps explain why not all young, bilingually raised children speak two languages. Lanza (1997b, 321324) compared mother-child interactions involving Siri, on the one hand, and Tomas, on the other. Through monolingual discourse strategies and her use of only English, Siri's mother negotiated a monolingual context with Siri. Lanza $(1988,75)$ notes, "Siri's mother was competent in Norwegian, but the role of being a bilingual can be negotiated in ongoing interaction as 


\section{Annick De Houwer and Janice Nakamura}

the mother feigns the role of a monolingual speaker". Siri responded to her mother's monolingual discourse strategies by speaking mostly English with her, although Siri could also speak Norwegian. Tomas' mother negotiated a bilingual context with Tomas through her use of bilingual discourse strategies. Furthermore, she spoke mainly English but also some Norwegian. Tomas hardly spoke any English with her.

While other factors also contribute to children's active bilingual use (e.g., amount and source of language exposure; De Houwer 2020), Lanza (1992, 1997b, 2004) proposed that parental discourse strategies (henceforth simply: strategies) constitute negotiations that socialize children into speaking a particular language. As such, they are important in developing active bilingualism. Many have argued that each parents' use of a single but different language consistently to their children (in what is known as the one parent, one language (1P/1L) setting) is crucial for fostering children's use of two languages. However, the actual experiences of many parents using $1 \mathrm{P} / 1 \mathrm{~L}$ can be quite different. Many are puzzled and upset to find that children speak only a single language, viz. the societal language (SocLang) used at (pre-)school, and that they understand but do not speak the nonsocietal language (NonSocLang). A large survey (De Houwer 2007) showed that only $73 \%$ of $1 \mathrm{P} / 1 \mathrm{~L}$ families had children who spoke both languages at home.

Lanza's hypothesis regarding the role of BIFIM in early bilingual development was based on just two 2-year-olds. Below we explore additional studies that have either implicitly or explicitly addressed BIFIM and its role in encouraging the use of two languages. We expand the coverage to studies of bilingually or trilingually raised children between ages 1 and 12 .

BFLA children exposed to the SocLang from birth usually speak that language, so it is not 'at risk' (De Houwer 2009). This explains our focus on what strategies, if any, can support children's use of the NonSocLang. Most studies also focus on the NonSocLang.

\section{Observational Studies of Parent-Child Interaction in Language Contact Situations}

This section reviews research on strategies and child bilingual use. We focus on observational studies with micro-level analyses of dyadic parent-child interactions. Dyadic interactions most clearly reveal how individual children are socialized in terms of language choice (cf. Lanza 2001). Table 2.1 shows a selection of studies arranged according to BFLA children's ages at the start of data collection, i.e., before age 2, between ages 2 and 4, and starting at age 4 . Some BFLA children started hearing a third language later. Studies observed adult-child interactions for periods between 1 and 35 months. When adults are said to speak a particular language, this mostly refers to the NonSocLang they habitually spoke to children. Most adults also understood (and possibly spoke) the SocLang. 

요

$$
\text { M. }
$$

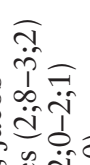

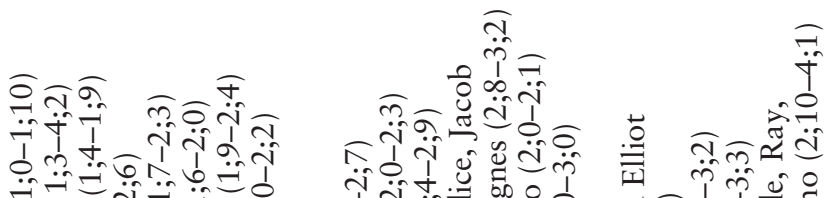

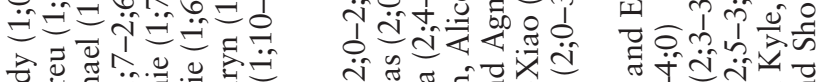

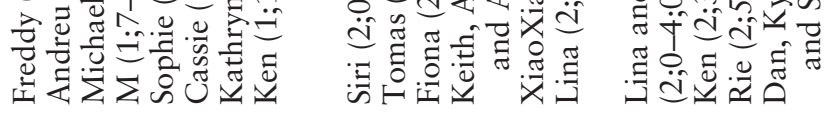

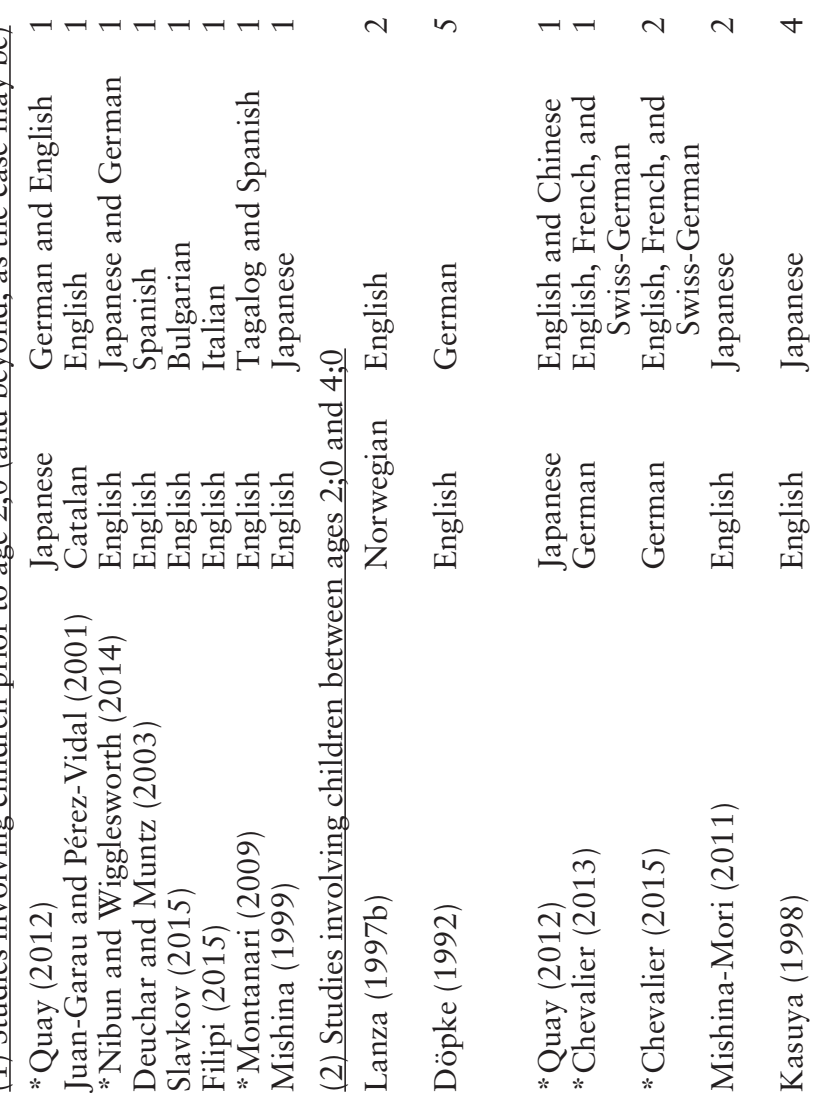




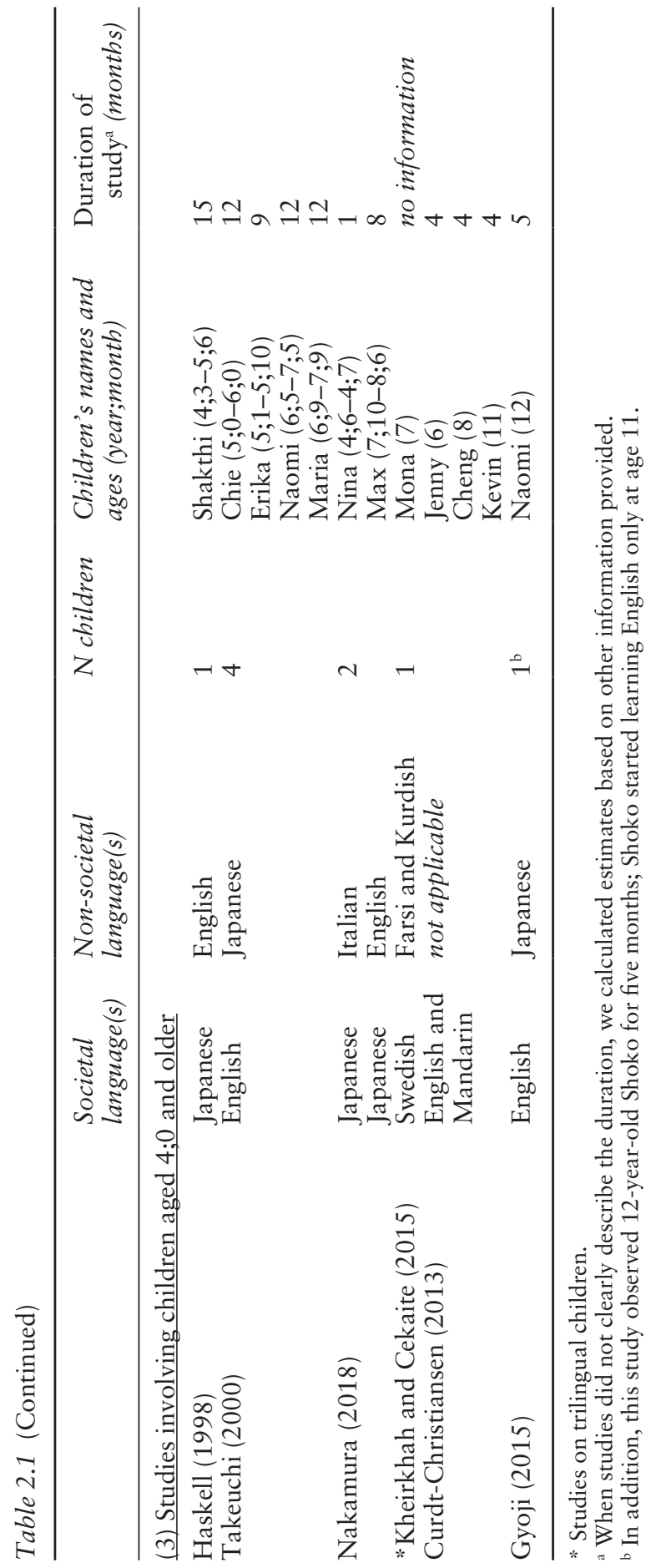


Some children well under the age of 2 can already adjust their language choice to their interlocutor (e.g., Sinka and Schelletter 1998). However, given that in the second year of life children are just learning to speak, many still need to learn when to use which language. Discourse strategies may play a crucial role in these early stages by socializing children into using habitual patterns of language choice. At age 1;6 English-Italian Cassie was unresponsive to her English-speaking father's use of MGS and AR, but just one month later she was able to produce the English words after he modeled them (Filipi 2015). Well before German-Japanese Michael turned two, his parents most frequently used MGS and never switched languages when he used the unexpected language (Nibun and Wigglesworth 2014). In response, Michael generally spoke the expected language, i.e., German to his father and Japanese to his mother. Although he knew many more Japanese than German words, he seldom used Japanese words with his father. This was possibly related to a combination of strategies: When Michael used Japanese, his father would first engage MGS. When Michael could not repair his utterance, paternal EGS or AR followed to provide him with the necessary vocabulary. By using these strategies, Michael's father signaled that he did not wish Michael to speak Japanese while providing him with the necessary input for producing German.

Use of AR can also encourage the use of the expected language. Adults interacting with Spanish-English M used AR quite a bit after M produced mixed utterances or the unexpected language (Deuchar and Muntz 2003). M's usual reaction after AR was to switch to the expected language. In contrast, after the monolingual strategy MGS, the child mainly continued to use mixed utterances or the unexpected language; unlike most other parents in the studies in Table 2.1, M's parents both routinely spoke both languages to her, depending on where they were. (Before age 1;11 M used mixed utterances or the unexpected language in about $25 \%$ of her utterances; after this age that proportion dropped to only about $10 \%$; see Table 2.4 in Deuchar and Muntz 2003).

The frequent use of bilingual strategies may encourage young children to choose Language Alpha even when they know the corresponding words in Language A. At 1;9, English-Spanish-Tagalog Kathryn often said English words to her Spanish-speaking caregivers even when she knew the equivalent Spanish (Montanari 2009). Her caregivers paid no attention to these English words and responded mostly using AR or MOS, thus signaling to Kathryn that her English was understood and accepted. Likewise, English-Japanese Ken used English in nearly half the time in interactions with his Japanese-speaking mother, who tended to insert Ken's English words in her Japanese clarification requests, i.e., she used LS (Mishina 1999). In contrast, Ken's use of Japanese with his English-speaking father never exceeded $10 \%$ of his utterances. Ken's father usually engaged MGS when Ken used Japanese. When Ken did not respond to the MGS, his father changed the topic in his next turn. This maintained a monolingual context 
and may have contributed to Ken's even lower level of Japanese use with his father when he was older (see below).

The studies of children under the age of 2 thus generally support BIFIM. So do studies of children between age 2 and 4. Four English-Japanese boys' language choice was Japanese $45 \%$ to $68 \%$ of the time right after their Japanese-speaking parents used "explicit" strategies, including MGS, but was only $11 \%$ to $52 \%$ of the time after "implicit" strategies, including AR and MOV, were used (Table 2.9, Kasuya 1998). In Chevalier (2013, 2015), trilingual Lina's English-speaking aunt's predominant use of monolingual (MGS and EG) and neutral (AR) strategies (41\% and $44 \%$ of strategies used respectively) likely contributed to Lina's active English production. More than half of Lina's utterances to her aunt were in English. Contrastively, Lina's French-speaking father used monolingual strategies minimally $(7 \%$ of discourse strategies). His frequent use of bilingual strategies, i.e., MOS and CS (45\% and $13 \%$ of strategies used respectively) failed to encourage French production; only 13\% of Lina's utterances to him were in French. Likewise, Ken's parents' contrasting use of strategies from early on (see above) is reflected in Ken's use of English about half of the time with his Japanese-speaking mother and his nearly absent use of Japanese with his English-speaking father after age 2 (Mishina-Mori 2011). At this point, Ken's father hardly needed to engage any monolingual strategies because Ken hardly spoke any Japanese with him. Rie, another child studied by Mishina-Mori (2011), hardly spoke any English to her Japanese-speaking mother. Her mother's use of MGS likely helped Rie make the expected language choice.

The effect of strategies on child language choice is most clearly elucidated by Juan-Garau and Pérez-Vidal (2001). They demonstrated how a change in parental use from bilingual to monolingual strategies can dramatically increase NonSocLang use. Between ages 1;3 and 2;5, CatalanEnglish Andreu frequently spoke Catalan with his English-speaking father, who relied heavily on MOS, thus negotiating a bilingual context. However, starting from when Andreu was 2;5, his father intentionally started increasing MGS. Questions such as How does daddy say that?, which were most frequently engaged between ages $3 ; 1$ and 3;3, dramatically reduced Andreu's Catalan use with his father, particularly from age 3;5 onwards. Whenever Andreu was unable to respond to MGS, his father followed up with EGS or AR (cf. Nibun and Wigglesworth 2014). Furthermore, Andreu was happy to accept that a puppet spoke just English. With the puppet's help, Andreu's father managed to get Andreu to speak more English. These monolingual strategies had a lasting effect even though they were used intensively for only a few months. After age 3;3, Andreu hardly addressed his father in Catalan even when the latter did not use monolingual strategies as often as he had before. This suggests that earlier used monolingual strategies may help establish a long-term pattern of parent-child interaction in the NonSocLang. Earlier, Taeschner (1983) also documented how her 
Italian-German pre-school-age daughters spoke more German to her after she used more MGS.

BIFIM also plays a role for children aged 4 and up. Haskell's (1998) examples suggest a link between English-Japanese Shakthi's frequent use of Japanese with her English-speaking father and his tendency to use MOS, which established a bilingual context. Discourse strategies were apparently able to counter the effect of increased exposure to English at school in four older English-Japanese children with mothers who consistently spoke Japanese to them (Takeuchi 2000). While MGS was rarely used and not found to be effective, maternal use of EGS or AR was quite successful in eliciting Japanese from the children's next conversational turn. For three of these children (ages 5 to 7), the occasional maternal use of MOS did not matter much because they rarely spoke English and were already actively interacting with their mothers in Japanese. However, for the 5-year-old who spoke more English, her mother's frequent use of bilingual strategies did little to promote her Japanese use. The girl only replied in Japanese 19.4\% and $3.3 \%$ of the time that MOS and LS were used, respectively.

Data from an unusual longitudinal and observational large study confirm that strategies are important even for school-aged children. Based on analyses of parent-child story-telling interaction in bilingual families in the United States, Park et al. (2012) traced the use and proficiency in Cantonese or Mandarin by 68 children who were 6 years old at Time 1 and nearly 7.5 years old at Time2. Parents who used monolingual strategies and consistently spoke a NonSocLang with children at Time1 had children who were proficient in that language at Time2. Parents who used more bilingual strategies at Time1 had children with limited proficiency in the NonSocLang at Time2. Furthermore, parents of children who were less proficient in the NonSocLang at Time1 provided less support for the NonSocLang at Time2. Park et al.'s (2012) study shows the many transactional processes (see Section 3.1 below) transpiring in parent-child interaction over time.

MOS may become a permanent feature of parental discourse style with older receptive bilingual children (children who understand two or more languages but speak only one). Nakamura (2018) studied two such children (ages 4 and 7). They used mostly Japanese with their Italian-speaking and English-speaking fathers, respectively. Regardless of the fathers' consistency in speaking the NonSocLang with their children, the children mainly spoke Japanese, the SocLang. The fathers' prevalent use of MOS perpetuated duallingual interactions. The few monolingual strategies that they used did little to elicit a NonSocLang response. Likewise, but now for Japanese as the NonSocLang, 12-year-old Naomi spoke almost exclusively English with her Japanese-speaking mother, who used MOS whenever Naomi spoke English, resulting in mostly dual-lingual conversations (Gyoji 2015).

The case studies of BFLA children discussed so far suggest that the use of mostly bilingual strategies by NonSocLang-speaking parents with young children hampers children's NonSocLang development, thus confirming 
Lanza's (e.g., 1997b) results for Tomas. Meng and Miyamoto's (2012) study of 2-year-old Xin, who had first only heard Chinese and after a move to Japan started hearing Japanese through daycare, suggests that strategies also play a role for sequential bilingual children. Although Chinese was the home language, Xin's mother's frequent use of MOS after Xin's Japanese utterances led to only $20 \%$ child production of Chinese utterances by the time she was 3 . A unique longitudinal study of children who had supposedly only heard Ukrainian from birth (see Section 3.2) but added English from daycare and school suggests that bilingual strategies can affect long-term NonSocLang maintenance. Ten mothers of 3-year-olds often used MOS when children spoke English to them (Chumak-Horbatsch 1987). At age 3, the children spoke Ukrainian at age-appropriate levels. At age 13, the same children were very close to losing Ukrainian (Chumak-Horbatsch 1999). Chumak-Horbatsch blames this partly on the use of some English in the home in the pre-school years and on mothers using bilingual strategies at that time.

As Taeschner (1983) and Juan-Garau and Pérez-Vidal (2001) showed, a switch to monolingual strategies can help turn things around and encourage NonSocLang use in BFLA pre-schoolers. The case studies in Table 2.1 do not furnish sufficient information for conclusions as regards older children. As we discuss below, children's agency may be an important factor here.

\section{Factors Moderating Parents' Use of Discourse Strategies}

Section 2 shows how BIFIM contributes to bilingual children's NonSocLang use and development. Here we explore what factors might help explain parents' use of bilingual rather than monolingual strategies in interactions with children.

\subsection{Transactional Parent and Child Effects}

Bornstein's $(2009,145)$ three-term transactional model of child development and parenting posits that "the child changes the parent and is in turn changed by the changed parent, or the parent changes the child who in turn changes the parent". Given this transactional model, the use of parental discourse strategies is also considered transactional: Its use is crucially and fundamentally about parents' language behavior in relation to their children's and vice versa.

Transactional relations are present from the beginning. BFLA infants may already react to parental language choice. Four-month-old bilinguals responded annoyedly when their mother, who had spoken Portuguese to them all day, addressed their father in Swedish at the end of the day (CruzFerreira 2006, 62). Arnberg $(1979,109)$ describes how a Swedish-English infant placed his hands over his Swedish-speaking father's mouth whenever he tried to speak English. Three-year-old Dutch-English Susan cried when 
her Dutch-speaking mother inadvertently addressed her in English, leading her mother to quickly switch back to Dutch (De Houwer 2009). Another 3-year-old, Finnish-Swedish Sara, refused to accept her Finnish-speaking father's Swedish utterance and declared that Swedish was her mother's language (Palviainen and Boyd 2013).

Very young bilingual children learn everything from scratch, including awareness about language choice. Lanza (1988) pointed out that parents who ask children for translations are fostering their bilingual awareness: "the metalinguistic input in various strategies used by the parents may contribute to bilingual awareness in these children" (Lanza 1988, 82). This developing awareness may affect children's responsiveness to strategies. Children must know that it is their language choice that is being queried when parents use MGS. By age 2 and a half most actively bilingual children are able to repair their language choice after being prompted (De Houwer 2017). Section 2 shows that some children can do so much earlier, but other children apparently cannot. Low awareness may be one reason why some children do not repair their language choice when prompted. Two-year-old Tomas did not interpret the few instances of MGS used by his mother as a cue to switch to English (Lanza 2004). Likewise, even at the much older age of 4;7, receptive bilingual Nina treated her Englishspeaking father's occasional use of EGS as genuine clarification requests and did not repair her Japanese utterances (Nakamura 2018). The "plurifunctionality of requests for clarification" (Lanza 2004, 305), coupled with parents' limited use of monolingual strategies, likely makes some children less aware that clarification requests may be prompts to switch languages.

Children may also actively resist such prompts. At around 1;7, after starting English daycare, Sophie ignored her Bulgarian-speaking father's requests for translation of her English into Bulgarian, a language she had spoken quite well for her age earlier. Instead, she moved on with the conversation, declined invitations to switch languages, and displayed negative emotions whenever her father used monolingual strategies (Slavkov 2015). In contrast to Sophie, Japanese-Mandarin-English Xiaoxiao spoke three languages at age 2;0 despite having a Chinese-speaking mother who predominantly used MOS in response to Xiaoxiao's use of Japanese and mixed utterances (Quay 2012). Unlike Quay's other trilingual subject, Freddy, who preferred to speak Japanese both at home and in daycare, Xiaoxiao was more eager to please her parents. Quay surmised that child personality and socio-psychological factors are one possible explanation for Xiaoxiao's willingness to accommodate her parents by using their respective languages. For slightly older Japanese-Mandarin-English trilingual Y (age 2;6), such factors were probably also at play. Y was fully aware that her grandparents were monolingual Mandarin speakers, but used non-verbal cues to help them decode her non-Mandarin speech and only switched to Mandarin as a last resort (Zhan 2020). The underlying child-related factors contributing 
to young children's accommodation or resistance to their interlocutors' language choice need further investigation.

At older ages, children exercise greater agency related to language choice. Primary school-aged children growing up with Kinyarwandan and French responded to their parents' use of Kinyarwandan just through French, the SocLang (Gafaranga 2010; this study concerns mainly multi-party interactions). At ages 5 and 6, children used MGSs themselves, e.g., the French question quoi? (what?). This question mirrored parental MGSs and signaled the children's preference for French. In comprehension checks, a 7-yearold used an EGS or AR by repeating a parent's Kinyarwandan utterance in French. Seven-year-old trilingual Mona (Kheirkhah and Cekaite 2015) often complied with her parents' insistence that she speak just the NonSocLang (Farsi or Kurdish), requests for translations and explicit teaching of the NonSocLang (see also Meyer Pitton's (2013) examples of dinner conversations in three Russian-French families in Switzerland). However, Mona also occasionally showed negative affect where she insisted on using Swedish, the SocLang, and explicitly stated that she did not know Farsi and Kurdish well enough. Her parents, eager to keep family life harmonious, often stopped insisting on NonSocLang use when Mona got angry. In less tense situations, they would pretend they did not understand a Swedish word Mona used in order to prompt her to say the word in the NonSocLang. Naomi, 12 years old, was able to articulate why she used English or Japanese, citing phonological or semantic factors. In interactions with her Japanese-speaking mother, she used mainly English but also some Japanese, against her mother's wish for purely Japanese interactions (Gyoji 2015). Her mother did not argue the point but persisted in using Japanese.

In a study of 44 Chinese-American families with pre-schoolers in the United States, Kuo (1974) noted that parents may use more English (LS) rather than just Chinese in response to children speaking more English. In addition to children's language choice per se, their use of passive resistance to the NonSocLang from a young age through monolingual strategies favoring the SocLang and at older ages through more explicit negotiations of language choice shows that their developing agency may counter parental strategies aimed at supporting the NonSocLang. Children's monolingual strategies favoring the SocLang thus may push parents to speak the SocLang too (Gafaranga 2010). However, children who earlier exercised their agency and rejected the NonSocLang may later regret that they are unable to speak the NonSocLang (Nakamura 2020). In young adulthood, some may ask their parents to speak the NonSocLang to them or may enroll in NonSocLang classes.

Despite showing resistance to the NonSocLang, children may use it selectively for achieving specific communicative goals. Said and Zhu's (2017) study of multi-party family interaction in the United Kingdom involving two brothers aged 6 and 9 showed how they used Arabic to gain the attention of their English-and-Arabic-speaking parents and to challenge their 
mother's authority. Likewise, an English-Gaelic 7-year-old used Gaelic, the NonSocLang, to request something, attract attention, or mitigate admonishments (Smith-Christmas 2016). Thus, as Lanza (e.g., 2004, 333) also acknowledged, children are co-constructive agents in socialization contexts for language choice. Children's developing maturity may help determine how successful they are in pushing their own agenda. However, parents' reactions to children's agency are just as important. These reactions will partly depend on parental levels of language awareness (see below).

\subsection{Parental Language Awareness}

As a bilingual investigator, De Houwer (1983) had to make a conscious effort to only speak Dutch to Kate. When Kate addressed her in English in an early recording, De Houwer responded in English twice before switching to Dutch. As an investigator, she was acutely aware of linguistic choices. However, caregivers may be quite unaware of their linguistic choices or the strategies they use (Lanza 1997b). This helps to explain why there may be a mismatch between what language(s) parents say they speak to children and what they actually speak. The ten mothers interviewed by ChumakHorbatsch (1987) firmly believed that they only spoke Ukrainian at home. Yet extended recordings of mother-child interactions in the home showed quite a bit of English present in mothers' language use, of which they were unaware. Moreover, mothers paid little attention to their children's English use and always used MOS.

Not only may parents be unaware of their language choice behavior. They may also be unaware that their language choice behavior may matter. This point is elaborated in the next subsection.

\subsection{Parental Beliefs and Other Factors}

While the studies reviewed in Section 2 underscore the importance of monolingual strategies for encouraging child bilingualism, the extent to which parents use such strategies is arguably affected by their impact belief or the conviction that they can "exercise some sort of control over their children's linguistic functioning" (De Houwer 1999, 83). Parents with a strong impact belief may engage monolingual strategies more often to influence children's language choice. Parents who use bilingual strategies may have a weak(er) impact belief; that is, they may be unaware that how they talk to children can influence their bilingual development.

Parents of English-Japanese bilingual children in Japan who had a strong impact belief reported that they spoke English consistently to their children and expected their children to do the same, likely signaling this through monolingual strategies (Nakamura 2019). Yet, despite having a strong impact belief, some parents may decide not to press the issue of language choice because they are worried about communication breakdowns. 
The fathers in Nakamura (2018) prioritized communication with their receptive bilingual children, so they interacted with them dual-lingually. However, parents who use monolingual strategies do not report communication failures; neither do the observational examples from children's reactions to MGS or EGS give the impression that communication was hampered.

Parents have different perspectives on their role in children's development and have different general parenting styles, varying between leaving children free to do whatever they like and being quite authoritarian. Parenting styles also affect the degree to which parents use specific strategies. Styles of parenting and corresponding use of strategies may be related to culture. For instance, while EGS is a common caregiver style of interaction in North American middle-class monolingual families, Western Samoan caregivers do not engage it because they tend not to participate in perspective-taking when talking to children (Ochs 1984).

\section{BIFIM and Research on Input, Family Language Policy, and Language Maintenance}

Our discussion so far has confirmed the importance of Lanza's (e.g., 1997b) foundational ideas on the link between parent-child interaction and children's bilingual production. One would think that this area of research belongs to both the more psycholinguistically oriented field studying language input in bilingual acquisition and the more sociolinguistically oriented field known as Family Language Policy. Surprisingly, neither of those fields pays much attention to it. Ours is the first overarching review of BIFIM. Explanations for why children do not speak a NonSocLang should also interest scholars working on intergenerational language maintenance. Also, in that field there is little attention to BIFIM.

It is quite puzzling that none of the three research fields pay much attention to BIFIM. The reason might have to do with methodological difficulties: After all, strategies can only be studied based on time-consuming observations of natural interaction. Yet, in observational studies (mostly in the bilingual acquisition literature), there is hardly any attention to discourse strategies. Do we find any reference to BIFIM in the general literature on bilingual parenting? We consider this in Section 5.

\section{Parental Discourse Strategies in Bilingual Parenting Resources}

Bilingual parenting resources have become more widely available than ever before. Many parents refer to them when making bilingual parenting decisions. Such advice can thus influence children's bilingual development. As reviewed above, studies addressing BIFIM have demonstrated how parental discourse strategies can help promote children's active use of the 
NonSocLang (in addition to the SocLang). Are parents generally informed that their discourse strategies may affect children's bilingualism?

An important but now-defunct channel, the Bilingual Family Newsletter (see Table 2.2, Part 2.2.1), made very little reference to parental discourse strategies, and none to BIFIM. In contrast, an early internet-based article commissioned by the Center for Applied Linguistics in Washington DC (De Houwer 1999, Table 2.2, Part 2.2.4) endorsed monolingual strategies and compared them to other common parental socialization efforts:

If you feel strongly about your children using one particular language with you, encourage them to use it in all of their communication with you. Try to discourage their use of another language with you by asking them to repeat what they said in the preferred language or by gently offering them the appropriate words in the language you want them to use. It is no more cruel than asking your child to say "please" before giving her a cookie. [our emphasis]

There are also numerous books on bilingual parenting written by academics and non-academics alike (the latter may be carrying out independent research). Some inform parents of empirical findings from child bilingualism research. Others rely mainly on anecdotes, parent interviews, and/or personal experience. To determine the extent to which BIFIM has been disseminated as bilingual parenting advice, we reviewed 23 English books and 11 books written in Dutch, French, German, Japanese, and Spanish (listed in Table 2.2, Parts 2.2.2 and 2.2.3, respectively). We focused on publications after 2000 to make sure authors had had a chance to refer to Lanza's 1992 and 1997 BIFIM publications.

Only 6 of the 33 books expressly mentioned BIFIM (bold titles, Table 2.2). In her popular book, Elke Montanari (2002) explained how parents can coax young children into responding in the expected language and strongly endorsed the use of MGS. The parents she interviewed also found other methods helpful, such as asking children to please speak language $\mathrm{X}$, because, as they explained to children, it matters to parents. This strategy does not feature in BIFIM, perhaps because Lanza's 2-year-old subjects were too young to understand requests which require a fair amount of metalinguistic awareness. Orioni (2015, $96 \mathrm{ff}$.) likewise presented MGS as an important strategy for fostering children's speech in two languages. Use of MGS worked well with her children, and they still complied with her use of MGS in their teens. Child bilingualism scholar Barbara Pearson (2008, 149-151) strongly endorsed BIFIM and suggested parents "may have to insist" (p. 151) on children speaking the "right" language, much like when they insist on music lessons. Several parents surveyed for her book reported that their children still readily accepted the fiction that their parents did not understand the language they happened to use. Barron-Hauwaert (2004, 14) suggested that AR would work well with younger children who are 


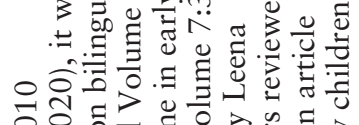

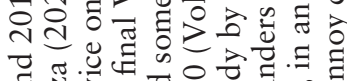

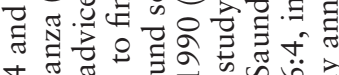

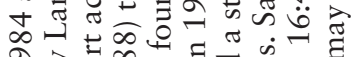

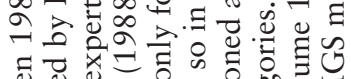

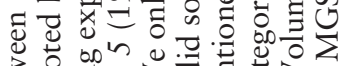

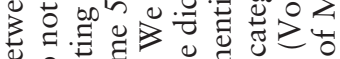

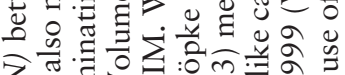

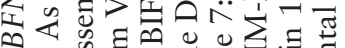

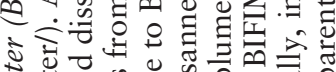

ป

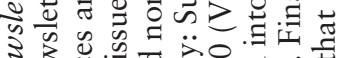

s

z

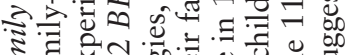

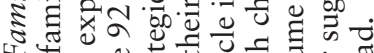

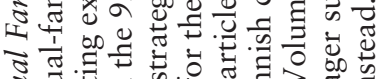

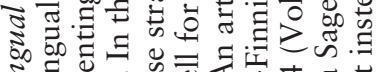

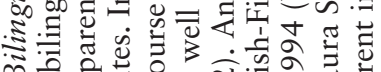

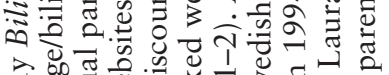

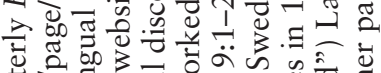

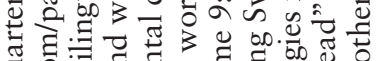

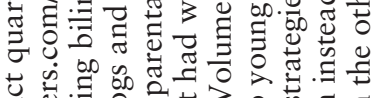

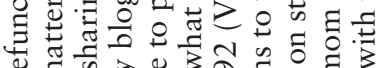

ช क के

$\frac{\overrightarrow{0}}{\frac{5}{5}}$

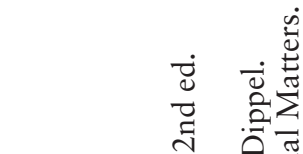

गं

ปี

峁

$\sum^{\pi}$

졸

莺

寻

苑

雚

व்

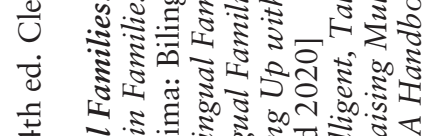

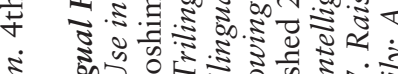

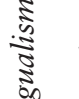

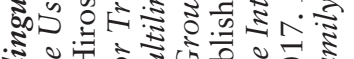
苟 इ के

i⿱

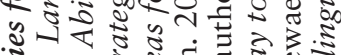
कूष

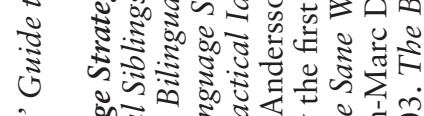

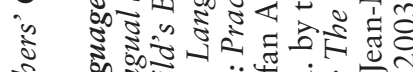

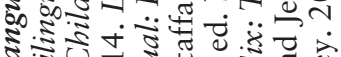

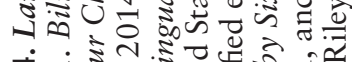

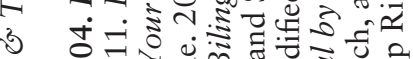
พ

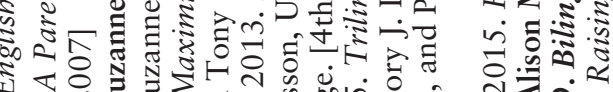

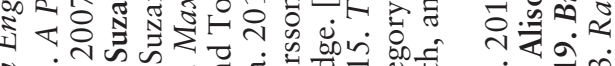

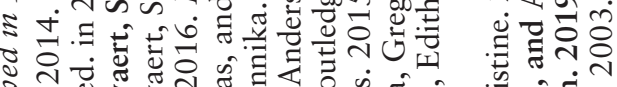

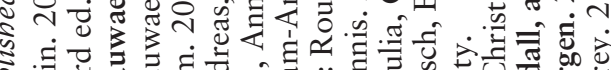

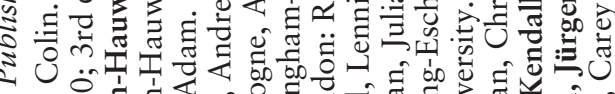

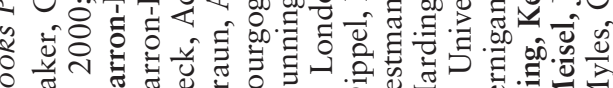
的

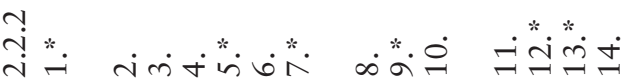




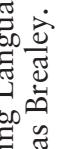

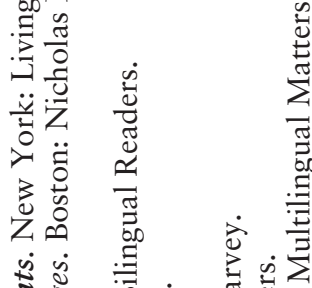

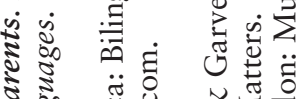

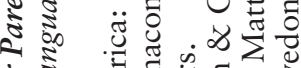

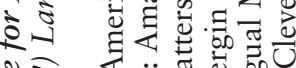

这市

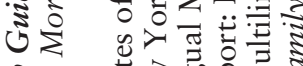

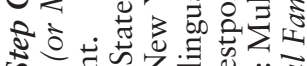
के วิ

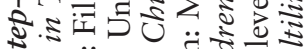

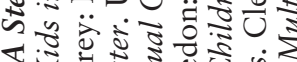

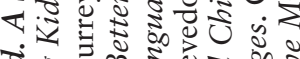
i.

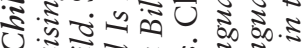

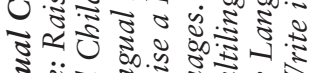

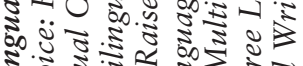

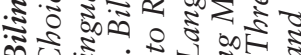

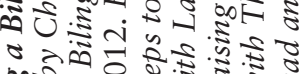

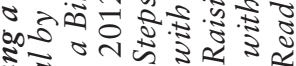

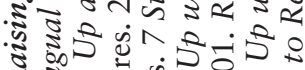

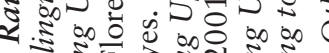

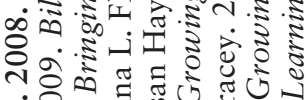

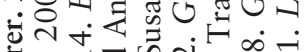

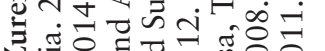

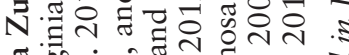
팹.

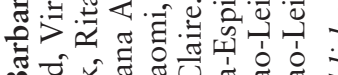

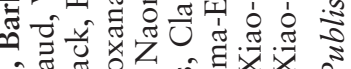

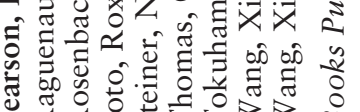

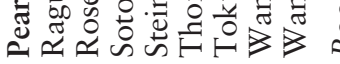

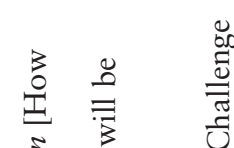

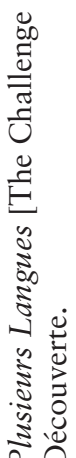

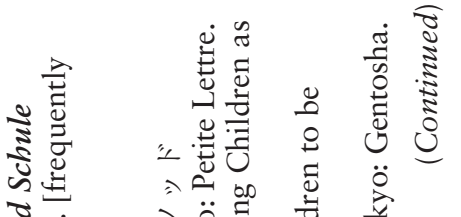

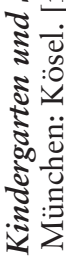

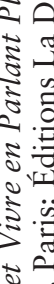

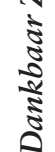

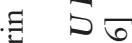

되 $\overline{\mathrm{J}}$

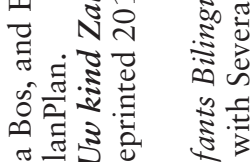

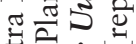

远落

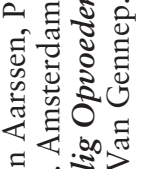

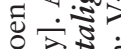

啳击苛

की

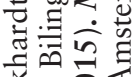

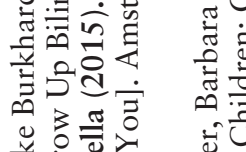

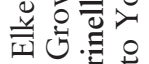

过.

ชี

必宁

0

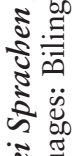

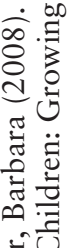

声

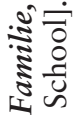

.

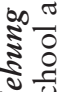

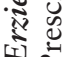

1

일

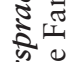

क्षे

$\sum^{2} \cdot \Xi$

章

इับ

อิำ

离

交示前

ᄋํㅇㄷㅠ

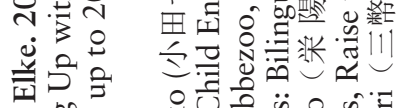

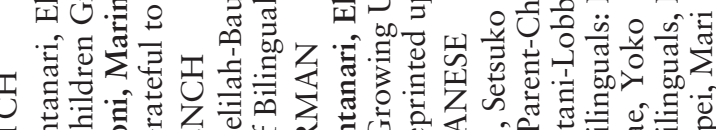

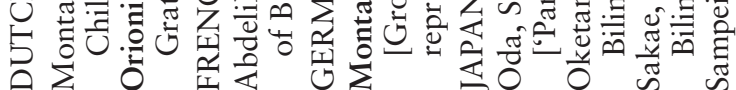

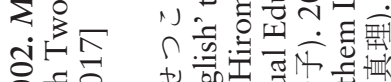

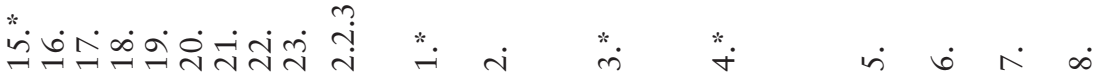




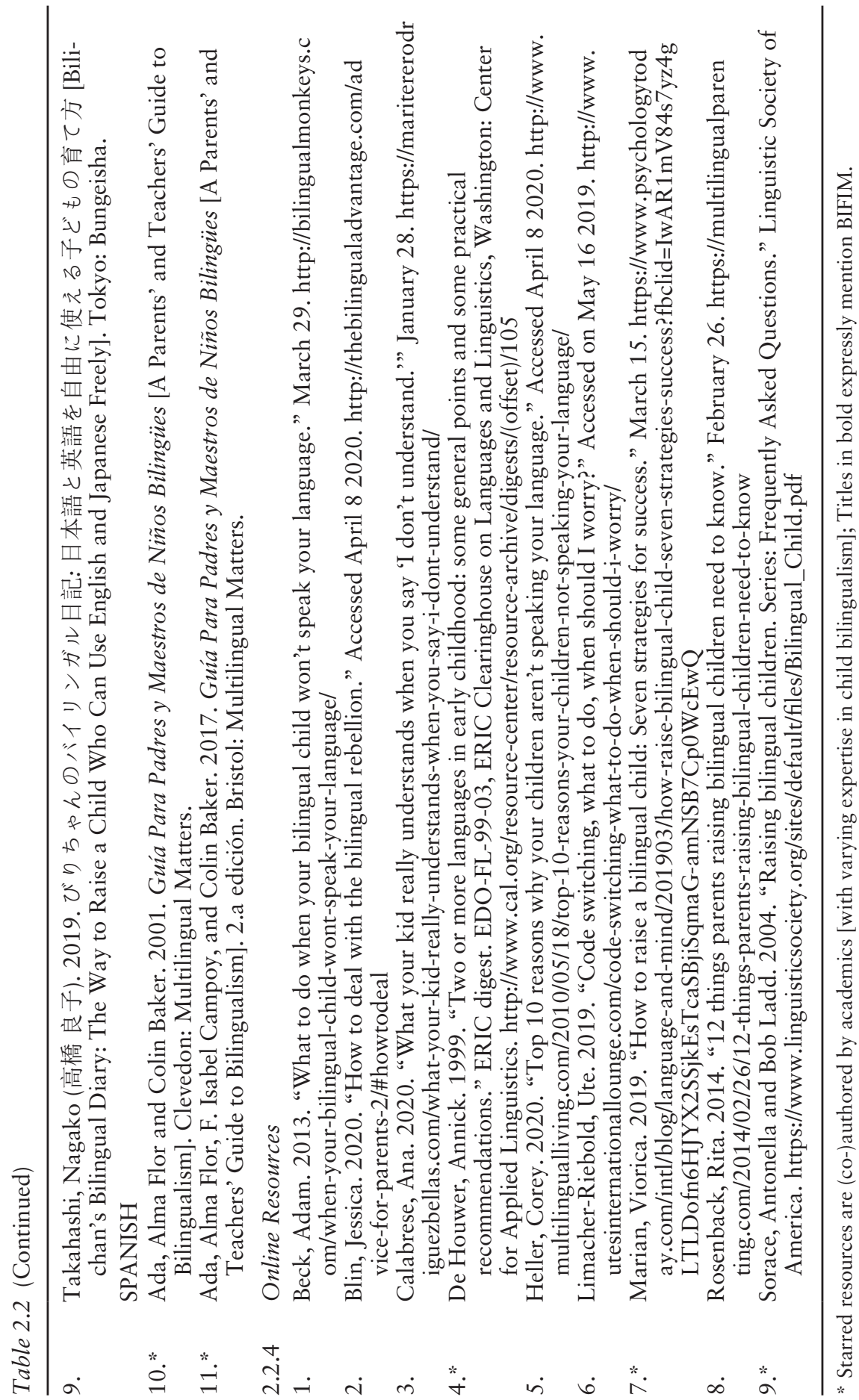


"absorbing language" and MGS and EGS are more suited to older children to get them to speak more of the NonSocLang. King and Mackey (2007) gave a lot of attention to BIFIM but mainly highlighted the discrepancy between parents' stated usual language choice and the reality in actual conversation. They suggested in a very non-committal way that Lanza's monolingual strategies are "worth a try" (p. 198). Child bilingualism scholar Meisel $(2019,121)$, however, did not recommend MGS, which he mainly interpreted as parents pretending not to understand a language. He argued that this may not reflect parents' bilingual reality and that children may feel "deceived" once they discover their parents are bilingual.

We also reviewed a few online sources (see Table 2.2, Part 2.2.4). Apart from De Houwer (1999), these did not mention BIFIM at all. Even bilingualism scholars with easy access to Lanza's work did not mention it (see the information leaflet of the Linguistic Society of America written by Sorace and Ladd [2004] and a blog by Marian [2019]). Thus, BIFIM has hardly been disseminated in bilingual parenting resources.

However, many sources provided some advice on how to deal with children's inappropriate language choices. Like Meisel (2019), many cautioned against the use of MGS (but offered no empirical data that warrants such negative advice). Parents are mostly advised to just continue using the NonSocLang when children speak the SocLang. Baker $(2014,94)$ even warned that "it is often impossible and unwise to compel a child to speak a language". This opinion also appeared in Spanish editions of his influential book (Ada and Baker 2001; Ada, Campoy, and Baker 2017). Some sources suggested not responding to children or even punishing them for speaking the "wrong" language. Needless to say, we do not endorse such practices. On the other hand, some sources suggested praising children for speaking the right language. Generally, advice is given without much attention to children's developmental stage.

\section{Discussion and Conclusion}

In 1988 Lanza wrote: “Careful study is needed of different parents' discourse strategies and how parental strategies change over time in relation to the child's language development" (p. 83). More than three decades hence we have only about 20 case studies that looked at strategies used with BFLA children, Park et al.'s (2012) longitudinal structured observations of 64 families, and Chumak-Horbatsch's $(1987,1999)$ suggestive study of ten families. Even though this database of observational studies of dyadic parent-child interaction with children aged 1 to 12 is still quite limited, our review has confirmed BIFIM's validity and the predictions it makes for active bilingualism in both younger and older children.

Lanza $(2001,227)$ wrote that "developmental and interactional perspectives are essential for determining the extent to which parental discourse strategies have an impact on the child's language choice" and that children 
are "active, creative social agents" $(2004,333)$. Bornstein's (2009) transactional model recognizes that both parents and children bring distinctive characteristics to each interaction and change as a result of it. The bidirectionality of interactions implies that children employ strategies of their own to socialize parents into using the language of their choice: Children's agency may influence the degree to which parents will use particular strategies, which in turn affects children's language choice. The degree to which child agency plays a role probably relates to parents' general parenting beliefs and language-related impact beliefs.

Further research is needed to understand how both parents and children simultaneously engage strategies to negotiate language choice from the early stages onwards. Yet is it the transactional nature of the developing parentchild relationship within an evolving environment affecting all parties that is likely the reason why monolingual strategies are often (1) hard to put into practice, and (2) may fail to produce lasting results. It is difficult to combat children's developing attitudes favoring the SocLang as a result of schooling (De Houwer 2020). The role of these developing attitudes needs further investigation.

Research so far has only scratched the surface of the transactional dynamics of discourse strategies in bilingual families. Many important issues remain unexplored. For instance, how decisive are earlier patterns of parent-child interaction for later child bilingual use? We know that pre-schoolers may be socialized into speaking two languages when earlier they spoke mainly one. Is it possible to reverse older children's single language use at home? Or is child agency just too strong after, say, age 5? Developmental perspectives are rarely discussed, even though MGS may work differently with 2-yearolds than with 10-year-olds. Most studies so far have focused on strategies used by one parent in mostly dual-parent households. What about the other parent's role? Especially in multi-party family interaction, the other parent's role may be quite fundamental (Venables, Eisenchlas, and Schalley 2014). Furthermore, most studies are limited to families mainly using 1P/1L. Do monolingual strategies work similarly when the same parent uses them in different languages (and in different settings)? Another issue relates to sibling rank. Home interaction patterns and family dynamics change when a sibling is born. How does this affect strategies used with firstborns, with younger children, and, later, among siblings? Many more questions remain.

As Section 5 shows, Lanza's seminal ideas about BIFIM have not been given much attention in bilingual parenting resources. Some writers seem to be unaware of BIFIM and the studies which support it. This may explain the common advice to parents to simply continue speaking the NonSocLang in response to children speaking the SocLang. Thus, the authors are actually advising the use of MOS, a bilingual strategy, which in fact does not encourage NonSocLang use. At the same time, the advice to continue speaking the NonSocLang is important encouragement because if parents switch to the SocLang, children will be even less likely to use the NonSocLang. However, 
while modeling the NonSocLang is necessary, it is insufficient to support children's active NonSocLang use, particularly when parents frequently use MOS (Nakamura 2018).

Parents who expose their children to two languages from birth usually expect them to speak both languages. The advice to keep speaking the NonSocLang and use MOS may lead to disappointment once parents find children unable or unwilling to speak the NonSocLang. Even so, some authors of bilingual parenting books reassure parents (without giving any evidence) that receptive bilingualism can be activated at an older age, for instance, through travel (Cunningham-Andersson and Andersson 2004; Jernigan 2015 in Table 2.2, Part 2.2.2). While there are two case studies of active-turned-receptive bilingual children who reactivated the NonSocLang on trips to the country where this language was the SocLang (Slavkov 2015; Uribe de Kellett 2002), not everyone has the chance to make such trips. Furthermore, it is hard to encourage NonSocLang production when active bilingualism has not been established from the beginning (Nakamura 2018).

More than four decades ago, many parents in Arnberg (1979) complained there were insufficient bilingual parenting resources. Parents now have access to much more bilingual parenting information. However, the importance of monolingual strategies that Lanza identified for supporting bilingual development is still insufficiently recognized. The lack of coverage means that parents are inadequately informed about the role of BIFIM. Gaps between bilingual parenting books and research findings also exist for other aspects of family bilingualism (Nakamura 2021). As Lanza (2020) has also pointed out, further research is needed to determine to what extent research evidence is reflected in bilingual parenting advice. Given the likely influence of bilingual parenting resources on parental language practices and thereby on the language outcomes of many young children raised in bilingual settings, researchers and authors of parenting resources need to communicate research-based insights to parents. The Harmonious Bilingualism Network (www.habilnet.org) is one of just a few initiatives disseminating research-based findings, including those of BIFIM, to the general public. However, more outreach work is needed to help families raise bilingual children.

\section{Note}

1 All terms that are ambiguous as to whether they relate to language, citizenship, or nationality refer to language unless otherwise indicated.

\section{References}

Arnberg, Lenore. 1979. "Language Strategies in Mixed Nationality Families." Scandinavian Journal of Psychology 20 (1): 105-112. doi:10.1111/j.1467-9450. 1979.tb00689.x 


\section{Annick De Houwer and Janice Nakamura}

Bornstein, Marc H. 2009. "Toward a Model of Culture $\leftrightarrow$ Parent $\leftrightarrow$ Child Transactions." In The Transactional Model of Development: How Children and Contexts Shape Each Other, edited by Arnold Sameroff, 139-161. Washington, DC: American Psychological Association.

Chevalier, Sarah. 2013. "Caregiver Responses to the Language Mixing of a Young Trilingual.” Multilingua 32 (1): 1-32. doi:10.1515/multi-2013-0001

Chevalier, Sarah. 2015. Trilingual Language Acquisition: Contextual Factors Influencing Active Trilingualism in Early Childhood. Amsterdam: John Benjamins. doi:10.1075/tilar.16

Chumak-Horbatsch, Roma. 1987. "Language Use in the Ukrainian Home: A Toronto Sample." International Journal of the Sociology of Language 63: 99118. doi:10.1515/ijsl.1987.63.99

Chumak-Horbatsch, Roma. 1999. "Language Change in the Ukrainian Home: From Transmission to Maintenance to the Beginnings of Loss." Canadian Ethnic Studies 31 (2): 61-74.

Cruz-Ferreira, Madalena. 2006. Three is a Crowd? Acquiring Portuguese in a Trilingual Environment. Clevedon: Multilingual Matters.

Curdt-Christiansen, Xiao Lan. 2013. "Negotiating Family Language Policy: Doing Homework." In Successful Family Language Policy: Parents, Children and Educators in interaction, edited by Mila Schwartz and Anna Verschik, 277-295. Dordrecht: Springer. doi:10.1007/978-94-007-7753-8

De Houwer, Annick. 1983. "Some Aspects of the Simultaneous Acquisition of Dutch and English by a Three-Year-Old Child." Nottingham Linguistic Circular 12: 106-129.

De Houwer, Annick. 1999. "Environmental Factors in Early Bilingual Development: The Role of Parental Beliefs and Attitudes." In Bilingualism and Migration, edited by Guus Extra and Ludo Verhoeven, 75-95. Berlin: Mouton de Gruyter. doi:10.1515/9783110807820

De Houwer, Annick. 2007. "Parental Language Input Patterns and Children's Bilingual Use." Applied Psycholinguistics 28 (3): 411-424. doi:10.1017/ S0142716407070221

De Houwer, Annick. 2009. Bilingual First Language Acquisition. Bristol: Multilingual Matters.

De Houwer, Annick. 2017. "Early Multilingualism and Language Awareness." In Language Awareness and Multilingualism (Vol. 6, Encyclopedia of Language and Education), edited by Jasone Cenoz, Durk Gorter, and Stephen May, 83-97. Berlin: Springer. doi: 10.1007/978-3-319-02240-6_6

De Houwer, Annick. 2019. "Language Choice in Bilingual Interaction." In The Cambridge Handbook of Bilingualism, edited by Annick De Houwer and Lourdes Ortega, 324-348. Cambridge: Cambridge University Press. doi:10.1017/9781316831922

De Houwer, Annick. 2020. "Why Do So Many Children Who Hear Two Languages Speak Just a Single Language?" Zeitschrift für Interkulturellen Fremdsprachenunterricht 25 (1): 7-26.

Deuchar, Margaret and Rachel Muntz. 2003. "Factors Accounting for Code-mixing in an Early Developing Bilingual." In (In)vulnerable Domains in Multilingualism, edited by Natascha Müller, 161-190. Philadelphia: John Benjamins. doi:10.1075/ hsm. 1 
Filipi, Anna. 2015. "The Development of Recipient Design in Bilingual Child-Parent Interaction." Research on Language and Social Interaction 48 (1): 100-119. doi: 10.1080/08351813.2015.993858

Gafaranga, Joseph. 2010. "Medium Request: Talking Language Shift into Being.” Language in Society 39 (2): 241-270. doi:10.1017/S0047404510000047

Gyoji, Eiko. 2015. “Children's Agency in Language Choice: A Case Study of Two Japanese-English Bilingual Children in London.” International Journal of Bilingual Education and Bilingualism 18 (6): 749-764. doi:10.1080/13670050. 2014.956043

Haskell, Dale. 1998. “The Influence of Context and Parental Input on Language Choice and Mixing in Bilingual Language Acquisition." Japan Journal of Multilingualism and Multiculturalism 4: 33-53.

Juan-Garau, Maria and Carmen Pérez-Vidal. 2001. "Mixing and Pragmatic Parental Strategies in Early Bilingual Acquisition.” Journal of Child Language 28 (1): 5986. doi:10.1017/S0305000900004591

Kasuya, Hiroko. 1998. "Determinants of Language Choice in Bilingual Children: The Role of Input." International Journal of Bilingualism 2 (3): 327-346. doi:10.1177/136700699800200304

Kheirkhah, Mina and Asta Cekaite. 2015. "Language Maintenance in a Multilingual Family: Informal Heritage Language Lessons in Parent-Child Interactions.” Multilingua 34 (3): 319-346. doi:10.1515/multi-2014-1020

Kuo, Eddie Chen-Yu. 1974. "The Family and Bilingual Socialization: A Sociolinguistic Study of a Sample of Chinese Children in the United States." The Journal of Social Psychology 92: 181-191. doi:10.1080/00224545.1974.9923096

Lanza, Elizabeth. 1987. "Code-mixing and Code-switching in the Bilingual Infant's Discourse." Paper presented at the Fourth International Congress for the Study of Child Language, Lund, July.

Lanza, Elizabeth. 1988. "Language Strategies in the Home: Linguistic Input and Infant Bilingualism." In Bilingualism and the Individual, edited by Anne Holmen, Elisabeth Hansen, Jørgen Gimbel, and J. Normann Jørgensen, 69-84. Clevedon: Multilingual Matters.

Lanza, Elizabeth. 1990. "Language Mixing in Infant Bilingualism: A Sociolinguistic Perspective.” Doctoral dissertation, Georgetown University.

Lanza, Elizabeth. 1992. "Can Bilingual Two-Year-Olds Code-switch?” Journal of Child Language 19 (3): 633-658. doi:10.1017/S0305000900011600

Lanza, Elizabeth. 1997a. "Language Contact in Bilingual Two-Year-Olds and Codeswitching: Language Encounters of a Different Kind?” International Journal of Bilingualism 1 (2): 135-162. doi:10.1177/136700699700100203

Lanza, Elizabeth. 1997b. Language Mixing in Infant Bilingualism. A Sociolinguistic Perspective. Oxford: Oxford University Press.

Lanza, Elizabeth. 2001. "Bilingual First Language Acquisition: A Discourse Perspective on Language Contact in Parent-Child Interactions." In Trends in Bilingual Acquisition, edited by Jasone Cenoz and Fred Genesee, 201-229. Amsterdam: John Benjamins. doi:10.1075/tilar.1

Lanza, Elizabeth. 2004. Language Mixing in Infant Bilingualism. A Sociolinguistic Perspective. Oxford: Oxford University Press.

Lanza, Elizabeth. 2020. “Digital Storytelling: Multilingual Parents' Blogs and Vlogs as Narratives of Family Language Policy.” In Språkreiser. Festskrift til Anne 


\section{Annick De Houwer and Janice Nakamura}

Golden, edited by Guri Bordal Steien and Lars Anders Kulbrandstad, 177-192. Sofiemyr: Novus forlag.

Meng, Hairong and Tadao Miyamoto. 2012. "Input and Output in Code Switching: A Case Study of a Japanese-Chinese Bilingual Infant." International Journal of Bilingual Education and Bilingualism 15 (4): 393-415. doi:10.1080/13670050. 2012.665826

Meyer Pitton, Liliane. 2013. “From Language Maintenance to Bilingual Parenting: Negotiating Behavior and Language Choice at the Dinner Table in BinationalBilingual Families.” Multilingua 32 (4): 507-526. doi:10.1515/multi-2013-0025

Mishina, Satomi. 1999. "The Role of Parental Input and Discourse Strategies in the Early Language Mixing of a Bilingual Child." Multilingua 18 (4): 1-30. doi:10.1515/mult.1999.18.4.317

Mishina-Mori, Satomi. 2011. "A Longitudinal Analysis of Language Choice in Bilingual Children: The Role of Parental Input and Interaction.” Journal of Pragmatics 43 (13): 3122-3138. doi:10.1016/j.pragma.2011.03.016

Montanari, Simona. 2009. "Pragmatic Differentiation in Early Trilingual Development." Journal of Child Language 36 (3): 597-627. doi:10.1017/ S0305000908009112

Nakamura, Janice. 2018. "Parents' Use of Discourse Strategies in Dual-Lingual Interactions with Receptive Bilingual Children." In Crosslinguistic Research in Monolingual and Bilingual Speech, edited by Elena Babatsouli, 181-200. Chania: ISMBS.

Nakamura, Janice. 2019. "Parents' Impact Belief in Raising Bilingual and Biliterate Children in Japan.” Psychology of Language and Communication 23 (1): 137161. doi:10.2478/plc-2019-0007

Nakamura, Janice. 2020. "Language Regrets: Mixed-Ethnic Children's Lost Opportunity for Minority Language Acquisition in Japan.” Multilingua 39 (2): 213-237. doi:10.1515/multi-2019-0040

Nakamura, Janice. 2021. "English Parenting for Japanese Parents: A critical review of advice in self-help books for raising bilingual children in Japan." English Today. doi:10.1017/S0266078421000286

Nibun, Yukari and Gillian Wigglesworth. 2014. "Early Pragmatic Differentiation in Japanese and German: A Case Study of a Developing Trilingual Child in Australia." International Journal of Multilingualism 11 (1): 76-96. doi:10.1080/ 14790718.2013.837910

Ochs, Elinor. 1984. "Clarification and Culture." In Georgetown University Roundtable 1984: Meaning, Form, and Use in Context: Linguistic Applications, edited by Deborah Schiffrin, 325-341. Washington, DC: Georgetown University.

Palviainen, Åsa and Sally Boyd. 2013. "Unity in Discourse, Diversity in Practice: The One Person One Language Policy in Bilingual Families." In Successful Family Language Policy: Parents, Children, and Educators in Interaction, edited by Mila Schwartz and Anna Verschik, 223-248. Dordrecht: Springer. doi:10.1007/978-94-007-7753-8

Park, Heejung, Kim M. Tsai, Lisa L. Liu, and Anna S. Lau. 2012. “Transactional Associations Between Supportive Family Climate and Young Children's Heritage Language Proficiency in Immigrant Families." International Journal of Behavioral Development 36 (3): 226-236. doi:10.1177/0165025412439842

Quay, Suzanne. 2012. "Discourse Practices of Trilingual Mothers: Effects on Minority Home Language Development in Japan.” International Journal of 
Bilingual Education and Bilingualism 15 (4): 435-453. doi:10.1080/13670050. 2012.665828

Said, Fatma and Hua Zhu. 2017. “No, no Maama! Say 'Shaatir ya Ouledee Shaatir'! Children's Agency in Language Use and Socialization." International Journal of Bilingualism 23 (3): 771-785. doi:10.1177/1367006916684919

Sinka, Indra and Christina Schelletter. 1998. "Morphosyntactic Development in Bilingual Children." International Journal of Bilingualism 2 (3): 301-326. doi:10.1177/136700699800200303

Slavkov, Nikolay. 2015. "Language Attrition and Reactivation in the Context of Bilingual First Language Acquisition." International Journal of Bilingual Education and Bilingualism 18 (6): 715-734. doi:10.1080/13670050.2014. 941785

Smith-Christmas, Cassie. 2016. Family Language Policy: Maintaining an Endangered Language at Home. Basingstoke: Palgrave Macmillan. doi:10.1057/97811375 21811

Taeschner, Traute. 1983. The Sun is Feminine: A Study on Language Acquisition in Bilingual Children. Berlin: Springer Verlag. doi:10.1007/978-3-642-48329-5

Takeuchi, Masae. 2000. “Japanese Parents' Discourse Strategies in Response to Inappropriate Language Choice by their Children." Japan Journal of Multilingualism and Multiculturalism 6: 20-44.

Uribe de Kellett, Angela. 2002. "The Recovery of a First Language: A Case Study of an English/Spanish Bilingual Child." International Journal of Bilingual Education and Bilingualism 5 (3): 162-181. doi:10.1080/13670050208667753

Venables, Elizabeth, Susana A. Eisenchlas, and Andrea C. Schalley. 2014. "OneParent-One-Language (OPOL) Families: Is the Majority Language-Speaking Parent Instrumental in the Minority Language Development?" International Journal of Bilingual Education and Bilingualism 17 (4): 429-448. doi:10.1080/ 13670050.2013.816263

Zhan, Ying. 2020. "The Role of Agency in the Language Choices of a Trilingual Two-Year-Old in Conversation with Monolingual Grandparents." First Language. doi:10.1177/0142723720923488 


\title{
3 The Role of the Input in the Acquisition of Code-Switching
}

\author{
Shannon Phillips and Margaret Deuchar
}

\section{Introduction}

This chapter will examine the little-studied question of how children acquire code-switching if they are brought up in a bilingual community where this is a common practice. We will focus on the relation between the input from the adult community of bilinguals and the child's (re)production of any code-switching patterns in the input. We define "code-switching" as the use of two or more languages in the same conversation and focus in this chapter on switching within an utterance. An excellent example from both adult and child speech is provided by Yip $(2013,134)$ from her study of a child acquiring Cantonese and English in Hong Kong. Example (1) is an utterance by an adult addressing a child:

(1) ngo5dei6 waan2-zo2 \# turtle sin1 laa1, hou2-mou2 aa3 we play-ASP turtle first SFP good-not-good SFP "Let's play with the turtle first, shall we?" (Yip 2013, 134)

In this example, the English noun turtle is inserted in an otherwise Cantonese utterance. In the reply by the child in Example (2), we can see that she mirrors the adult utterance by similarly inserting the English word turtle in an otherwise Cantonese utterance.

$\begin{array}{llllll}\text { (2) ngo5dei6 jau5 [/] jau5 } & \text { loeng5 } & \text { go3 turtle } & \text { gaa3 } \\ \text { we have have } & \text { two } & \text { CL } & \text { turtle } & \text { SFP } \\ \text { "We have two turtles!" } & \text { (Yip 2013, 134) } & \end{array}$

This general pattern of English insertions in Cantonese turns out to be common in the Cantonese/English bilingual community, and our chapter will address the question of how a child acquires the community switching patterns.

The data we shall analyse will be from another bilingual community, in Wales, UK. Although only about $20 \%$ of the population of Wales speak 
Welsh as well as English, bilingual speakers are concentrated in particular areas in the north and west of the country. We already know from data collected from adult bilingual speakers in these areas that they have a strong tendency to speak Welsh with each other and that about $10 \%$ of their speech involves intraclausal code-switching to English (see Deuchar, Webb-Davies, and Donnelly 2018, 90). Our research question asks how young developing bilinguals acquire these specific patterns and if they can be discerned in their earliest speech productions. Our analysis focuses on the data from six children in the CHILDES CIG1 corpus, a longitudinal study of Welsh/ English bilingual children between the ages of 1 and 3 (see Aldridge et al. 1998). We use the Matrix Language Frame (MLF; Myers-Scotton 2002) as a tool in comparing the structure of the mixed utterances (containing both Welsh and English) in the children's speech with that found in the adult community.

Attention to how linguistic input affects children's language acquisition was fairly sparse in many twentieth-century studies in which the Chomskyan assumption regarding the "poverty of the stimulus" (Chomsky 1980, 34) held sway. According to this assumption, the input played a relatively minor role compared with the language-specific mental predisposition that children were thought to bring to the language learning task. However, a few notable studies (e.g. Newport, Gleitman, and Gleitman 1977) did draw attention to the important role of parental input, and their insights were further built upon by the usage-based approach to language acquisition, which emerged towards the end of the twentieth century (cf. Langacker 1987; Behrens 2012). In the same period, there began to be increasing interest in the question of how children acquire more than one language, either from birth or in early childhood. Many case studies focusing on bilingual acquisition from birth appeared in the 1980s and 1990s (e.g. Hoffmann 1985; De Houwer 1990; Döpke 1992; Deuchar and Clark 1996; Köppe 1997). Because there were two languages in the input, attention was necessarily focused on who provided this input and in what quantity.

But most of these early bilingual acquisition case studies had two flaws from the perspective of the present study: they focused almost exclusively on parental input as opposed to input from the community, and in addition many of them explicitly or implicitly considered mixing or code-switching by parents to be undesirable and so did not study it in detail. The concern with mixing was that it might interfere with children's ability to separate the two languages being acquired. Thus there was often an unstated aim of ensuring that the children become as competent in their two languages as monolinguals in each language (see Grosjean's [1989] warning regarding this aim). This approach clearly ignores the possibility that code-switching may be a normal practice in the community and that these norms need to be acquired for children to become fully functioning members of the community. 


\section{Shannon Phillips and Margaret Deuchar}

\section{Code-Switching in Adults}

Over the past 40 years, code-switching has become one of the central topics of study for bilingualism research as people have realised that codeswitching is not an "isolated, quirky phenomenon but a widespread way of speaking" (Gardner-Chloros 2009, 9). Code-switching studies can act as a window into the nature and compatibility of the grammars of the world's languages. Chomsky's generative grammar emphasises the role of "competence" or I-language whereby language is the system that allows speakers to produce grammatical sentences (Chomsky 1986). Implied in this definition is the idea that each language forms a discrete system of rules. However, code-switching raises questions about how and when two systems are able to combine. Furthermore, code-switching offers insights into the processing constraints which lead people to prefer certain types of switches over others. Of particular interest within this field of structural code-switching research, and the type of code-switching considered here, is code-switching within individual clauses, otherwise known as "intraclausal" code-switching (cf. Deuchar 2012). Code-switching within the clause allows us to observe how the syntactic structures of the two languages interact.

The idea that code-switching can be governed by linguistic constraints emerged with the work of linguists such as Pfaff (1979) and Poplack (1980), both focusing on Spanish/English code-switching. Pfaff concluded that "Surface structures common to both languages are favoured for switches" (Pfaff 1979, 314), an important observation that is also reflected in Poplack's proposal of the "equivalence constraint" whereby speakers avoid code-switching constructions that violate the surface word order of either language involved in the mixed utterance (Poplack 1980, 581). Joshi (1985), studying Marathi/English code-switching, drew attention to the fact that code-switching between the two grammatical systems was governed by an asymmetric "switching rule" by which a speaker could switch from the language of the morpho-syntax within a clause (the Matrix Language) into the other language (the Embedded Language) but not vice versa (Joshi 1985, 192). Joshi's work formed the basis for the development of Myers-Scotton's (1993) Matrix Language Frame model. The components of the model are the "Matrix Language" (ML), which sets the morphosyntactic frame for mixed utterances, and the "Embedded Language" (EL), which provides the single lexemes and switched EL island constituents that are switched into the ML (Myers-Scotton 1993, 1). The main claim of the model is that "the ML sets the morphosyntactic frame for ML + EL constituents" (Myers-Scotton 1993, 7). The model specifies that the ML can be empirically verified on the basis of the two principles below (Myers-Scotton 1993, 83).

a. Morpheme Order Principle (MOP): in Matrix Language + Embedded Language constituents consisting of singly occurring Embedded 
Language lexemes and any number of Matrix Language morphemes, surface morpheme order (reflecting surface syntactic relations) will be that of the Matrix Language.

b. System Morpheme Principle (SMP): in Matrix Language + Embedded Language constituents, all system morphemes that have grammatical relations external to their head constituent (i.e. which participate in the sentence's thematic role grid) will come from the Matrix Language.

Myers-Scotton originally proposed the MLF on empirical grounds based on her study of Swahili/English speakers in Kenya (Myers-Scotton 1993), and the model has been successfully applied to many other language pairs (see, e.g. Carter et al. 2011; Hebblethwaite 2010; Ihemere 2016; Khan and Khalid 2018). These and other studies have shown us that asymmetry between the ML and EL is not only found within the clause but is also common across an entire corpus in that one of the two languages more frequently assumes the role of the ML. Hebblethwaite (2010) suggests that it is most often the lower status or minority language, which is most frequently the ML. Particularly relevant for the present study is the finding that in the Welsh/English bilingual community, the minority language Welsh is indeed the most frequent Matrix Language (cf. Deuchar, Webb-Davies, and Donnelly, 2018). However, few quantitative corpus-based studies have been done, although such studies are crucial to provide information regarding the input to children who are growing up in bilingual communities where codeswitching is common.

\section{Code-Switching in Children ${ }^{1}$}

Despite the fact that many of the early case studies of developing bilinguals did not study language mixing in detail, being more concerned with how children separated their two languages, there were a few studies that did focus on language mixing or switching in children. For example, Vihman (1985) noted that function words were more likely than content words to be mixed in early two-word utterances produced by a developing Estonian/English bilingual, and the same was reported by Deuchar (1999) in her study of Spanish/English bilingual acquisition (see also Deuchar and Vihman 2002, 2005).

Another approach was to consider whether code-switching in children appeared to be subject to the same constraints as adult code-switching, for example, testing the application of the MLF model outlined above to child speech. Paradis, Nicoladis, and Genesee (2000) took this approach to their bilingual child data, French/English data collected in Canada. They found that "the children obeyed all the constraints set out in the Matrix Language Frame model the majority of the time" (Paradis, Nicoladis, and Genesee 2000, 245). However, they did not relate the children's code-switching patterns directly to the input. Vihman (1998) tested the MLF model on 
Estonian/English data collected over four years in the USA from conversations between her two children, aged roughly 3-7 and 6-10 years, respectively. She found that the Matrix Language of utterances was either English or Estonian, but most frequently Estonian. She reported that "When we speak of CS [code-switching], then, we are essentially speaking of the use of English words and phrases within Estonian utterances" (Vihman 1998, 60). This preference for switching from Estonian to English rather than the reverse might have been due to English being the community language, but this possibility was not explored.

Other studies of bilingual development had also noted that some children's switching was more common from one of the languages to the other rather than the reverse. However, many investigators tended to ascribe such frequency differences in bilingual child speech to dominance or greater proficiency in one language than another, rather than to the greater frequency of one Matrix Language compared with another in the input. For example, Petersen (1988) proposed the "dominant-language hypothesis", according to which "in word-internal code-switching, grammatical morphemes of the dominant language may co-occur with lexical morphemes of either the dominant or the non-dominant language. However, grammatical morphemes of the non-dominant language may co-occur only with lexical morphemes of the non-dominant language" (Petersen 1988, 486). On the basis of the code-switching pattern in her data alone, Petersen concludes that the child's dominant language is English. A more rigorous test of the "dominant-language hypothesis" would require an independent measure of the child's language proficiency. Petersen does mention that the child (living in the USA) receives more input in English than in Danish, including from her peers, but takes this to support her assumptions regarding dominance rather than to consider the influence of input from the community. Lanza (1997a, 1997b) also finds frequency differences in the mixed utterances of two developing Norwegian-English bilinguals in Norway, Siri and Tomas. Siri exhibits more mixing than Tomas, but the mixed utterances of both generally show a Norwegian grammatical frame with English insertions. Lanza interprets this pattern as exhibiting their dominance in Norwegian in line with Petersen (1988). However, she also notes that Siri's data can be accounted for in terms of the Matrix Language Frame model (MyersScotton 2002) and that Norwegian can thus be described as the Matrix Language of Siri's mixed utterances.

What neither Petersen nor Lanza appears to consider is the potential role of the community in providing input in which one Matrix Language is more frequent than the other. The child in Petersen's study was living in the USA, where English can be assumed to be the majority language. In Lanza's study the children lived in Norway, where the insertion of English items into a Norwegian grammatical frame is a frequently attested pattern in adults (cf. Grimstad, Lohndal, and Åfarli 2014). Few studies have separated the role of dominance and of the community language in bilingual acquisition. An 
exception, however, is Patuto et al. (2014), who found that the community language had a greater influence on code-switching than individual dominance, measured by comparing the MLU (mean length of utterance) in the two languages.

Despite the lack of emphasis in research work on the role of the community language, there has been some attention to the role of the input by parents in the development of code-switching in children. An early pioneer in this area was Goodz (1989). She used recordings of both adult and child speech to investigate quantitatively the relation between parental mixing in the input and mixing in the children's productions. Goodz found positive correlations between the child's and the mother's code-mixing in three out of the four children, but only in one of the child's mixing in relation to the father's. More recently, Baker and Van den Bogaerde (2008) investigated the extent to which the combination of Netherlands Sign Language (NGT) and Dutch used by deaf parents when addressing their deaf children was used in the child productions. They found that when the parents used utterances that included both signs and spoken or mouthed words, NGT was most commonly the "base language" of such utterances, and this pattern was replicated in the deaf children's productions.

One of the first studies to shift attention away from parental input to that of non-parental interlocutors, was that by Comeau, Genesee, and LaPaquette (2003). They were able to demonstrate that French/English developing bilinguals in Canada varied their rates of mixing to reflect that of their interlocutors to a greater extent than that of their parents. One problem that arises in comparing the results of studies of this kind is that not all studies use the same definition of language mixing. Many studies share the practice of identifying utterances containing words from more than one language as mixed, but some also consider utterances to be mixed if they are in the non-designated language, i.e. not in the main language the adult is using to address the child. This problem is illustrated in the study by Yip and Matthews (2016) of children acquiring Cantonese and English in Hong Kong. In this study, they note that the children mostly follow the pattern common in the adult community of inserting English items into a Cantonese morphosyntactic frame (as in Example (2) above) rather than following the much rarer pattern of inserting Cantonese items into an English morphosyntactic frame. But if a child were to produce an utterance like Example (2) in a recording where the adult was speaking English, the utterance would have been coded by Yip and Matthews as involving mixing in an English context, despite the fact that it does not have an English morphosyntactic frame.

This problem could have been avoided by coding utterances as either unilingual or mixed and, if mixed, as having Matrix Language A or B. Such an approach was taken by Eichler, Hager, and Müller (2012) in their study of 16 developing bilinguals who were acquiring either German and a Romance language or two Romance languages. One child, Marie, was acquiring 
German and French in France and was recorded in both German and French "contexts", with the language of the context being that used by the adults. In a recording in which Marie was being addressed in German, she produced the utterance "c'est fini le bett" ("the bed is made") in which the German word Bett 'bed' has been inserted in a French morphosyntactic frame. If the "mixed" portion were identified as that in the non-designated language, then it would be c'est fini le, the French part of the utterance, although mixing of non-constituents in this way would be unusual. An alternative was to identify the utterance as having a French Matrix Language with a German insertion. In an analysis of mixed DPs (Determiner Phrases) like le bett, produced by the children, the investigators discovered that the determiner almost always matched the language of the verb, a common pattern in adult speech not widely recognised at the time of their study, but demonstrated later by Blokzijl, Deuchar, and Parafita Couto (2017). The approach by Eichler, Hager, and Müller (2012) paved the way for a recognition, pursued in the present study, that developing bilinguals, like adults, may combine languages in ways similar to the adults in their community, and that identification of mixing or switching should be based on syntactic criteria rather than on the language choice of the adult in the same conversation.

\section{The Present Study}

\subsection{Research Question}

Given the lack of attention in previous work to the way in which code-switching patterns in the adult community provide input to the acquisition of bilingual code-switching in children, our study makes use of both adult and child corpora from the same bilingual community in order to determine the extent to which the adult norms appear to be replicated in child speech. In particular, we shall be focusing on whether Welsh turns out to be the most frequent Matrix Language in child code-switching as well as adult code-switching.

\subsection{Data}

The corpus of adult data (known as Siarad, the Welsh word for "speak") was collected between 2005 and 2008 from 151 bilingual Welsh-English speakers in Wales, with the speakers being predominantly based in the north. It consists of 447,507 words in 40 hours of conversations between pairs or groups of speakers who were known to one another and is available publicly on the website bangortalk.org.uk and talkbank.org. Here can be found transcripts of the recordings in CHAT format (see MacWhinney 1991), including glosses and translations of the utterances into English. The recordings are also available. Further details regarding the method of data collection and the profile of the speakers are available in Deuchar, WebbDavies, and Donnelly (2018). 
The corpus of child data is known as the Welsh Acquisition Database (or CIG1) and was collected in 1996-1997 as part of a longitudinal study of children aged 18-30 months who were acquiring Welsh in the bilingual communities of north-west and mid-Wales (see Aldridge et al. 1998). General information about the corpus is available at https://childes.talk bank.org/access/Celtic/Welsh/CIG1.html. Here the investigators state that "All the parents (apart from one who learned Welsh from 3;0) were firstlanguage speakers of Welsh". We know that the parents all spoke English as well because virtually everyone in Wales knows English (cf. Gathercole and Thomas 2009) and because the transcripts show frequent code-switching into English by the adults. The data consists of 304,846 words in 84 hours of conversations recorded between seven individual child speakers and the researchers and/or family members. The children's pseudonyms and their ages during the study are listed in Table 3.1.

The two corpora were collected within about ten years of each other, meaning that we can consider the speech of adults in the two corpora to be comparable. About half of the speakers in the adult corpus were over 40 years old between 2005 and 2008, so we can assume their language patterns to be similar to those in the community providing input in the 1990s. Furthermore, the adults and children in both corpora were recorded in overlapping geographical areas.

Although we were able to use the analysis of code-switching in the Siarad corpus of adult speech conducted by Deuchar, Webb-Davies, and Donnelly (2018), we decided also to analyse the actual adult input to one of the children, Dewi, as represented by the recordings of his speech in interactions with adults. Our focus, as in the analysis of the child speech, was the distribution of the ML in mixed utterances. There were four adult speakers who addressed Dewi in the recordings of his speech: Mam - Dewi's mother, Dad - Dewi's father, and Susan and Alice - the investigators. In the recordings at least one investigator was present, and in the majority of the cases the investigator was alone with the child. ${ }^{2}$ All of the adult speakers were bilingual in Welsh and English. The scope of our study did not allow a detailed analysis of all the adult speech in the CIG1 corpus, but the speech sample provided by Dewi's interlocutors provides a sample of actual input

Table 3.1 CIG1 Child Speakers and Ages

\begin{tabular}{ll}
\hline Child pseudonym & Age \\
\hline Alaw & $1 ; 1.19-2 ; 3.21$ \\
Dewi & $1 ; 9.21-2 ; 6.9$ \\
Rhys & $1 ; 8.30-2 ; 5.21$ \\
Elin & $1 ; 5.8-1 ; 9.20$ \\
Bethan & $1 ; 7.28-2 ; 4.20$ \\
Melisa & $1 ; 6.20-2 ; 3.17$ \\
Rhian & $1 ; 6.17-2 ; 3.23$ \\
\hline
\end{tabular}


to a child which, like the data from Siarad could be compared with the speech of the children in CIG1.

\subsection{Method of Analysis of Adult Corpus Siarad (Deuchar, Webb-Davies, and Donnelly 2018)}

In order to identify the patterns of code-switching in the Siarad data, all clauses with finite verbs were extracted from the transcripts (available at bangortalk.org.uk). Each word in the transcripts had been coded as Welsh or English according to whether the word was (1) only to be found in a Welsh dictionary and therefore coded Welsh; (2) only to be found in an English dictionary and therefore coded English; and (3) to be found in both language dictionaries and therefore coded as ambiguous between Welsh and English. ${ }^{3}$ The clauses were coded for two types of information: (1) whether they were mixed or unilingual and (2) whether the Matrix Language was Welsh or English. Clauses containing words from both Welsh and English were coded as mixed, whereas clauses containing words from only one language were coded as either unilingual Welsh or unilingual English. The Matrix Language was identified according to the language of the finite verb, either Welsh or English. This was an operationalisation of the System Morpheme Principle, outlined above in Section 2. The Morpheme Order Principle was also relevant, but the System Morpheme Principle was prioritised as it turned out to be simpler to implement using automatic analysis. The method of automatic analysis is described by Deuchar, Webb-Davies, and Donnelly (2018, 86-92). The method allowed the authors to deal with a total of 66,428 clauses, both unilingual and mixed and to arrive at a quantitative overview of the distribution of types of clauses and their Matrix Language in the entire corpus of data. This distribution gives us an idea of the relative frequency of the various types of clauses and thus their patterns in the input to children acquiring Welsh and English.

\subsection{Method of Analysis of Child Data in CIG1 Corpus}

\subsubsection{Extracting the Data from the CIG1 Corpus}

In order to directly compare the mixing patterns in the children's speech with the mixing patterns in the adult speech reported by Deuchar, WebbDavies, and Donnelly (2018) we focused only on the mixed utterances in the CIG1 dataset.

A mixed-language utterance was defined as an utterance containing both English and Welsh words, so was always longer than one word. For three of the speakers (Bethan, Melisa, and Rhian) the mixed utterances were extracted manually. For the other child speakers (Alaw, Dewi, Elin, and Rhys), the English language tags (“@s:eng”, “@s”, “xs”, “xxs” in the transcripts) provided by the project researchers in these transcripts were only 
used to automatically extract any utterances with English material using the CLAN programme (MacWhinney 1991). Any of the extracted utterances that included only English material were then excluded from our mixed utterance dataset. For instance, utterances like Example (3) were classified as mixed and were thus included in our dataset for analysis while utterances like Example (4) were classified as English only and thus were not relevant for an analysis of code-switching.

(3) mae

o 'n mynd

i $\operatorname{crash}^{5}$

[Rhys020521 - Rhys]

be.V.3S.PRES PRON.3S.M PRT go.NONFIN to crash.NONFIN

"it is going to crash" 6

(4) swimming costume

[Melisa011106 - Melisa]

"swimming costume"

As part of our criteria for labelling an utterance as mixed, we specified that any English material (e.g. "toys" in Example (3)) had to be searched for in the Welsh dictionary ${ }^{7}$ in case it existed as a Welsh word, albeit borrowed from English. Lipstick in Example (5) below is a good example of an English word that has been borrowed into Welsh and appears in the Welsh dictionary as lipstic "lipstick". The English word lipstick and the Welsh word lipstic are phonologically similar, meaning that a given utterance of either word could not be marked confidently as either Welsh or English, and would be classified as linguistically ambiguous as explained in Section 4.3. Other linguistically ambiguous words included bello (Welsh belo), yeah (Welsh ie ), cement (Welsh sment), jeans (Welsh jîns), and yoghurt (Welsh iogwrt). Okay and come on were also treated as linguistically ambiguous as were commercial and proper names as in Examples (6) and (7) below.

(5) lipstick fi

[Alaw020212a - Alaw]

lipstick my.PRON.1S

"my lipstick"

(6) LCB yn mynd

[Rhys011108 - Rhys]

JCB PRT go.V

"JCB (excavator) go"

(7) mixer Dewi

[Dewi011018 - Dewi]

mixer Dewi

“Dewi's mixer”

Utterances like those in Examples (5)-(7), which did not contain at least two linguistically unambiguous words from both languages, were excluded 
from the dataset for analysis. In addition, potentially mixed utterances were excluded where the transcription indicated uncertainty about the utterance. See Examples (8) and (9) below. ${ }^{8}$

$$
\begin{aligned}
& \text { (8) < fish sy 'ma> [?] } \\
& \text { fish is this } \\
& \text { "this is a fish" } \\
& \text { (9) <xxx> [=? mae] doli xxx [=? yn] cheeky [Alaw020004 - Alaw] } \\
& \text { be.V.3S.PRES dolly PRT cheeky } \\
& \text { "the dolly is cheeky". }
\end{aligned}
$$

Despite the necessity for excluding various utterances from our dataset of mixed utterances, we still had a total ${ }^{9}$ of 300 mixed utterances to analyse, as outlined in Table 3.2.

\subsubsection{Method of Coding the Child Data}

All of the mixed-language utterances were coded for Matrix Language (Welsh or English). The Matrix Language was identified by applying the MOP and the SMP. This section outlines the specific points of contrast between Welsh and English grammar, which were used to determine the ML for the child CIG1 utterances, first for the MOP and then for the SMP.

The MOP could be applied to the CIG1 data to determine the ML when there was a divergence between the word order of Welsh and English, as in the example below of a modified noun phrase. Within noun phrases in English, the typical word order has a modifier, such as an adjective preceding the noun (10).

$$
\begin{aligned}
& \text { tough day } \\
& \text { "tough day" }
\end{aligned}
$$

Table 3.2 Child Mixed Utterances in CIG1

\begin{tabular}{lc}
\hline Speaker & Total Mixed Utterances \\
\hline Alaw & 60 \\
Dewi & 81 \\
Rhys & 118 \\
Elin & 14 \\
Bethan & 3 \\
Melisa & 13 \\
Rhian & 11 \\
Total: & 300 \\
\hline
\end{tabular}


Within noun phrases in Welsh, the typical word order is where the noun precedes a modifier such as an adjective, as shown in Example (11).

$$
\begin{aligned}
& \text { dynes hyll } \\
& \text { woman old.ADJ } \\
& \text { "an old woman" }
\end{aligned}
$$

[Alaw020317 - Alaw]

Because of the divergence in word order between English and Welsh, the order of the noun and the adjective in a noun phrase can be used to indicate the ML in mixed utterances using the MOP alone. This method is illustrated in the CIG1 data in Examples (12) and (13). The noun + adjective word order in (12) identifies the noun phrase as having a Welsh ML while in (13) the adjective + noun order indicates an English ML.

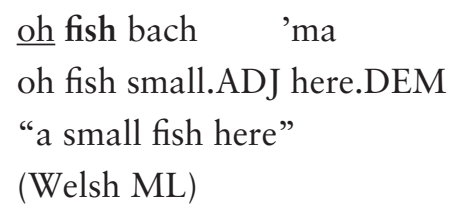

$$
\begin{aligned}
& \text { mawr fish } \\
& \text { big.ADJ fish } \\
& \text { “a big fish” } \\
& \text { (English ML) }
\end{aligned}
$$

[Dewi020523 - Dewi]

As previously outlined above, the SMP indicates that all system morphemes with grammatical relations external to their head constituent (including subject-verb agreement) will come from the Matrix Language. To identify the ML using the SMP in our mixed utterance dataset, we looked for the language of the subject-verb agreement. For instance, Example (14) has agreement between the singular third-person verb mae and the pronominal subject $O$.

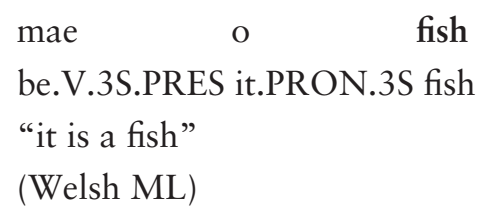

mae O fish

be.V.3S.PRES it.PRON.3S fish

"it is a fish"

(Welsh ML)
[Dewi20213 - Dewi]

Coding the ML was less straightforward for other utterances in our corpus. Because of the developmental nature of the data, verbal morphology was sometimes incomplete. For example, there is no verbal morphology available to analyse the ML of the utterances in Examples (15)-(16) by the SMP, 
nor does its word order differ from English in a way that would allow us to identify the ML by the MOP.

$$
\begin{array}{ll}
\text { mynd yn crash } \\
\text { go.V PRT } & \text { crash } \\
\text { "go crash" } &
\end{array}
$$

(16) catch yfana

[Dewi020126 - Dewi]

catch there

"catch(es) there"

In our analysis of utterances like the ones above, we chose to take the bare verb alone to indicate the ML of the utterance without insisting that the verb had to have finite marking and subject-verb agreement.

If an utterance did not have enough material to allow us to classify the ML according to either the MOP or the SMP, then we coded the ML as "Undetermined". This applied to Example (17). It is a mixed utterance, but there are no clues from the MOP because the order would be the same in English ("whoops-a-daisy again") and there's no verb, so we cannot classify the utterance using the SMP. $28.67 \%$ of all the mixed utterances included in our dataset had to be classified as Undetermined in this way.

$$
\begin{aligned}
& \text { woop a day eto } \\
& \text { whoops a daisy again } \\
& \text { "whoops-a-daisy again" }
\end{aligned}
$$

[Alaw200302 - Alaw]

\subsection{Method of Analysing the Adult Data in the CIG1 Corpus}

The CLAN programme was used to extract utterances that had been tagged by the original researcher as including English language material. If those utterances contained more than one clause, the mixed clause or clauses were identified and an ML assigned to each mixed clause. As with our analysis of the CIG1 child utterances, we applied the SMP and the MOP to determine the ML for the adult utterances. For example, the mixed clause in (18) was assigned Welsh as the ML. Following similar principles to those used in the child data, (19) is a unilingual clause which we excluded from the data set, and (20) was also excluded because the word helicopter could be either English or Welsh.

$$
\begin{aligned}
& \text { be mae hi 'n wneud yn kitchen? [Dewi011009- Dad] } \\
& \text { what be.V.3S.PRES she.PRON.F.3S PRT do.NONFIN in kitchen } \\
& \text { "what is she doing in the kitchen?" }
\end{aligned}
$$


(20) lle mae

helicopter

[Dewi0202172 - Sue]

where be.V.3S.PRES helicopter

"where is the helicopter?"

\section{Results}

\subsection{CIG1 Data Analysis (Child Speech)}

Figure 3.1 shows the ML distribution for all the child speakers across the corpus. Of all of the mixed utterances with determinable ML, $96.73 \%$ of the utterances had Welsh ML, while only $3.27 \%$ of the utterances had English ML. Figure 3.2 shows the distribution of ML for each individual speaker. All the mixed utterances with a determinable ML produced by Elin, Bethan, Melisa, and Rhian had a Welsh ML with no instances of English ML. This could be in part due to the smaller sample size for those four speakers. Elin had 11 utterances with a determinable ML, Bethan had 2, Melisa had 13, and Rhian had 10. Alaw, Dewi, and Rhys all had high levels of Welsh ML with $97.73 \%, 89.13 \%$, and $98.86 \%$ Welsh ML, respectively.

\subsection{Comparison with Siarad Data}

Figure 3.3 shows the distribution of the ML in the mixed utterances of the Siarad corpus (left-hand side) compared with the mixed clauses of the CIG1

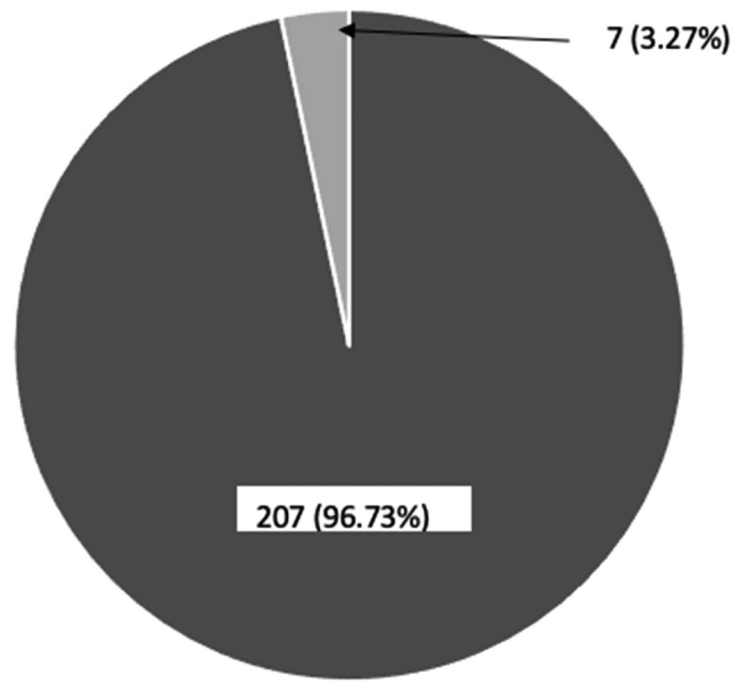

- Welsh ML $\quad$ English ML

Figure 3.1 Matrix language distribution in child mixed utterances 


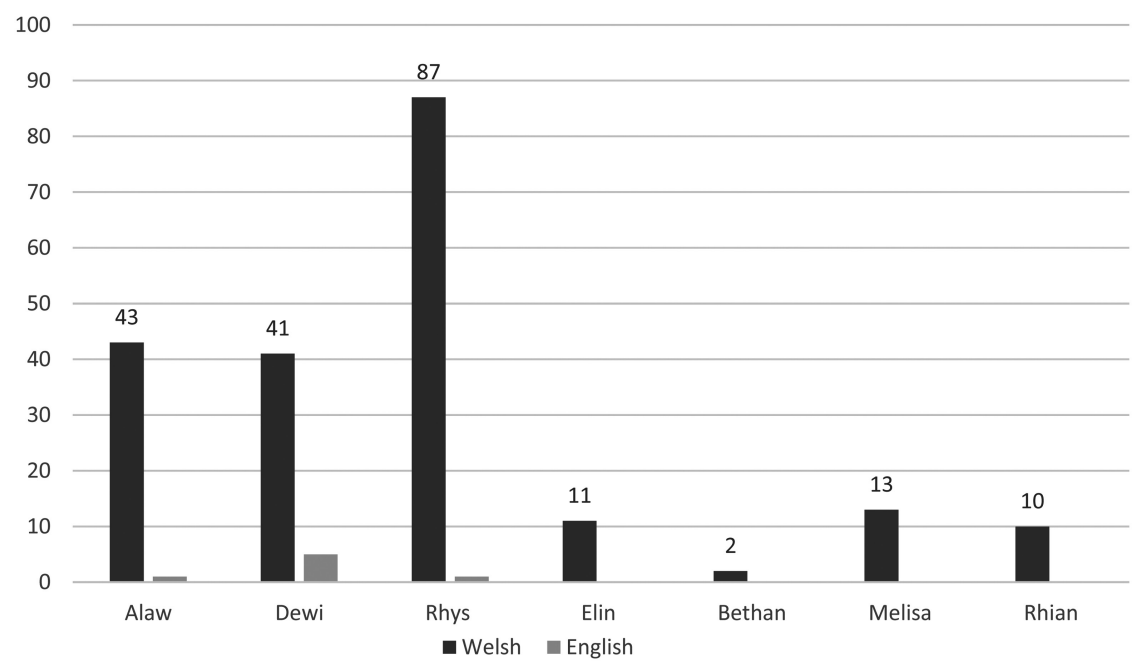

Figure 3.2 Matrix language distribution in individual child mixed utterances

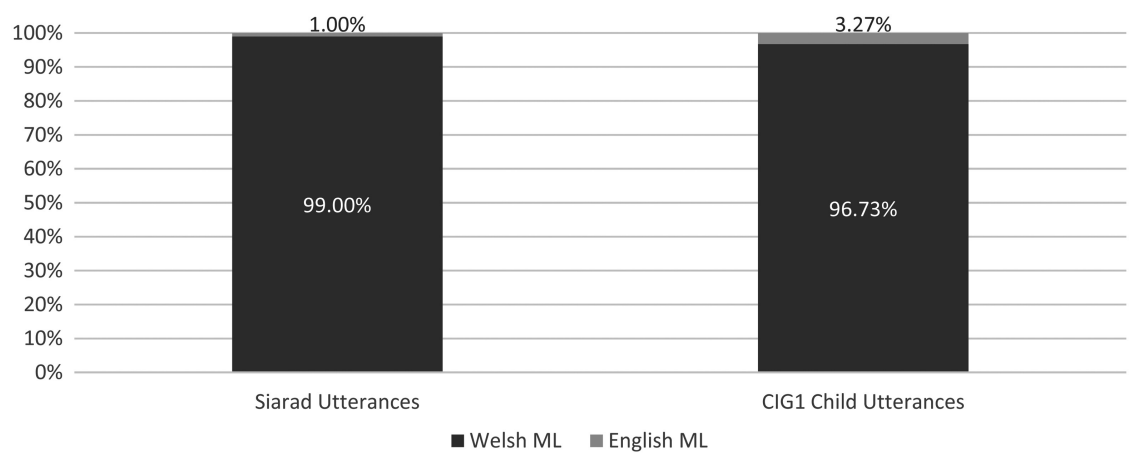

Figure 3.3 Matrix language distribution in Siarad adult corpus compared with child mixed utterances in CIG1

child corpus (right-hand side). In both figures cases of Undetermined ML are excluded. The Siarad data are based on Table 5.3 of Deuchar, WebbDavies, and Donnelly $(2018,90)$. There were 53 utterances with English ML and 5908 utterances with Welsh ML in the Siarad mixed data. There is a striking similarity between the ML distribution across the Siarad and the CIG1 corpora. For clauses with an assignable ML, 99\% had Welsh ML in Siarad and $96.73 \%$ had Welsh ML in the CIG1 child data.

\subsection{Comparison with Dewi Adult Data}

The results from the analysis of adult speech in the Dewi transcripts indicated a total of 126 mixed utterances with 108 Welsh ML, 1 English ML, 


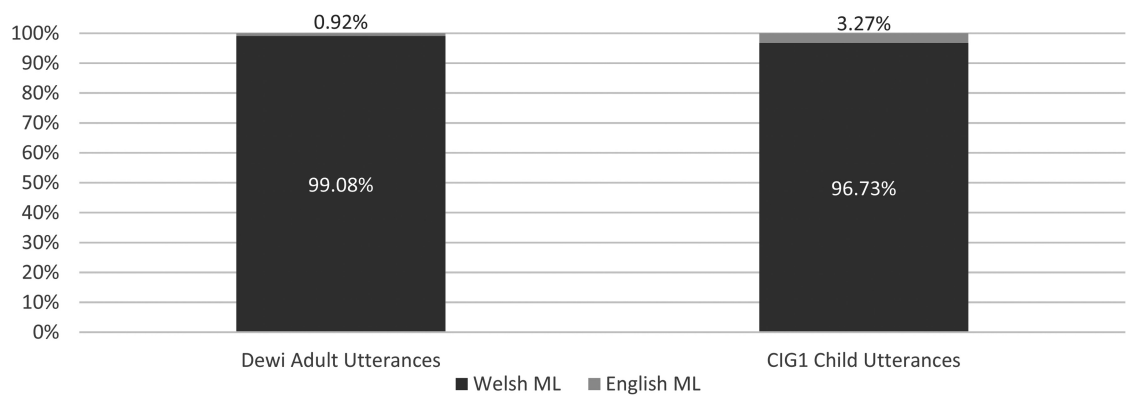

Figure 3.4 Matrix language distribution in mixed utterances by Dewi's adult interlocutors compared with child mixed utterances

and 17 Undetermined ML. There was a distribution of 99.08\% Welsh ML and $0.92 \%$ English ML (see Figure 3.4). Thus, in both the child CIG1 data and the adult data from the Dewi transcripts, Welsh is overwhelmingly the most frequent ML.

\subsection{Refining the Criteria: Child Mixed Utterances Containing Verbs}

One potential criticism which might be proposed of the methodology of analysis applied to the child data is that we took the language source of all verbs, whether inflected or not, as indicative of the SMP in identifying the Matrix Language of the utterance. So as outlined previously, the bare verb mynd "go" in Example (15) (repeated below) was taken to indicate Welsh subject-verb agreement because an equivalent adult utterance would have the verb as part of a periphrastic construction with a finite auxiliary verb.

$$
\begin{aligned}
& \text { mynd yn crash } \\
& \text { go.V PRT crash } \\
& \text { "go crash" }
\end{aligned}
$$

Example (21) provides an idea of how mynd might appear in an adult periphrastic construction with the auxiliary mae as the finite verb:

$$
\begin{aligned}
& \text { mae Postman Pat yn mynd i 'r gegin [Rhys020305-Sue] } \\
& \text { be.V.3S Postman Pat PRT go.NONFIN to the kitchen } \\
& \text { "Postman Pat goes to the kitchen" }
\end{aligned}
$$

However, we know from studies of Welsh language acquisition that such periphrastic constructions develop gradually in children and that they are preceded by shorter and simpler versions (see, e.g. Aldridge et al. 1998). Even in adult speech, the auxiliary is sometimes deleted, as studied in detail 


\section{Shannon Phillips and Margaret Deuchar}

by Davies and Deuchar (2014), so our use of bare verbs in Welsh to indicate the ML may be defensible. However, to ensure that this method did not unduly influence our results, we decided to conduct a second analysis of the child speech in which we allowed bare verbs to be considered equivalent to reduced periphrastic constructions only if the child had already shown evidence of producing full periphrastic constructions either in the same transcript as the occurrence of the bare verb or in one of an earlier recording.

For this second analysis we first isolated the mixed utterances with bare verbs but no finite marking as in Example (22).

$$
\begin{aligned}
& \text { wneud magic } \\
& \text { do.V magic } \\
& \text { "do magic" }
\end{aligned}
$$

[Rhys020027 - Rhys]

For these utterances, we introduced a criterion requiring that for a bare verb in a phrase to be considered equivalent to a reduced construction, there needed to be evidence of subject-verb agreement used by the child either within the same transcript or within a transcript recorded earlier in the child's development. If the child displayed earlier evidence of acquiring the full version of the proposed reduced construction, then we accepted the bare form as having a classifiable ML by the SMP.

For instance, in Example (23) there is no finite verb, only a bare verb mynd. This example was taken from transcript $020212 \mathrm{~b}$, recorded when Alaw was 2;2,12. However, in an earlier transcript 010119, recorded when Alaw was $1 ; 1,19$, she produced a full periphrastic construction (Example (24)), suggesting that she had already acquired the full construction. We therefore classified the utterance in (23) as having a Welsh ML based on the bare verb as we had done in our first analysis.

$$
\begin{aligned}
& \text { hwn yn mynd i crash rwan } \\
& \text { this PRT go.NONFIN to crash now } \\
& \text { "this is going to crash now" }
\end{aligned}
$$

$$
\begin{aligned}
& \text { mae car yn mynd i fana. [Alaw010119-Alaw] } \\
& \text { be.V.3S.PRES car PRT go.NONFIN to there } \\
& \text { "the car is going there" }
\end{aligned}
$$

Similarly, we applied this stricter criterion to the utterances with only bare verbs in English, requiring that children show evidence of the previous acquisition of forms with full subject-verb agreement. For instance, Example (25) contains a verb in English but has no overt subject-verb agreement. There was no evidence of the speaker Dewi producing any utterances with full subject-verb agreement in English in any of the earlier transcripts, so the 
utterance in (25), previously coded as English ML on the grounds that the verb was English, was now coded as Undetermined ML.

$$
\begin{aligned}
& \text { push na } \\
& \text { push not } \\
& \text { "no push" }
\end{aligned}
$$

[Dewi020227 - Dewi]

\subsubsection{Results}

Figure 3.5 below shows the results from the initial analysis in the left-hand column, which reports the same information as Figure 3.1 with $96.73 \%$ Welsh ML and 3.27\% English ML across the child mixed-language utterances in the corpus. The right-hand column shows the results of the second analysis applying a stricter criterion to utterances with bare verbs and no verbal morphology. For the second analysis there was an average of $98.50 \%$ Welsh ML and 1.50\% English ML. We can see from Figure 3.5 that the distribution of the ML in the two charts is very similar, suggesting that the application of the less strict criteria did not have a significant effect on the results.

\section{Discussion}

The principal question that this study sought to address was whether there was a similarity in the code-switching patterns of children and adults from the same speech community. The results presented here confirm that similarity. In the comparison of the CIG1 data with Deuchar, Webb-Davies, and Donnelly's (2018) analysis of Siarad, both samples showed an overwhelming majority of Welsh ML in mixed utterances. In other words, both the bilingual adults in the community and the developing bilingual children adopt similar code-switching patterns using their two languages, Welsh and English.

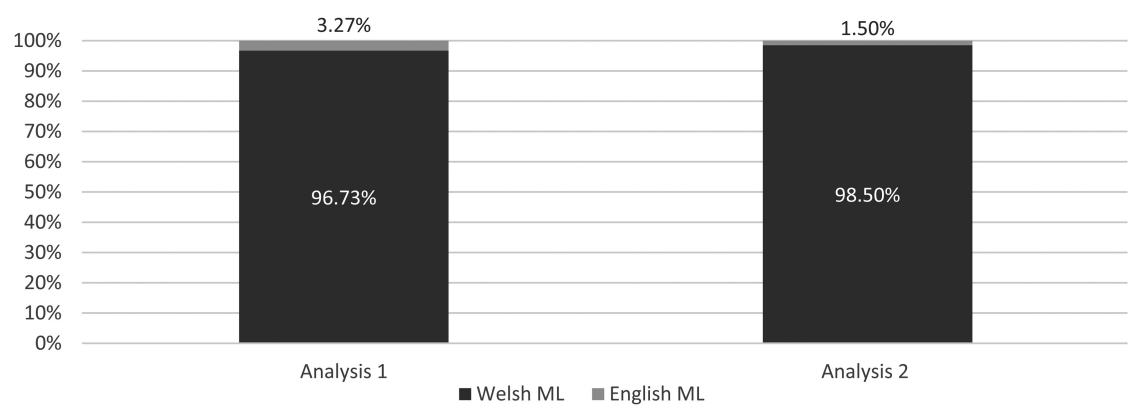

Figure 3.5 Matrix language distribution in child mixed utterances comparing Analysis 1 and Analysis 2 


\section{Shannon Phillips and Margaret Deuchar}

Our results show that the children adopt the same code-switching pattern as the adults, in which English words and phrases are inserted into a Welsh morphosyntactic frame in the overwhelming majority of their mixed utterances. The reverse pattern, which could theoretically occur, in which Welsh words or phrases might be inserted into an English morphosyntactic frame, is virtually absent.

The results of doing the same analysis on a small sample of the adults speaking on the CIG1 transcripts from the child speaker Dewi indicated a very similar pattern of results. These results show that the type of codeswitching input being received by the children in real time during the recordings had a Welsh syntactic frame the majority of the time. Because the Siarad corpus had a very similar pattern of ML distribution, we take these results as an indication that the Siarad corpus is a plausible proxy for the input to the child speakers in CIG1.

Overall, our results show that, even allowing for the developmental nature of child speech in such young children, their code-switching patterns reflect quite closely the speech of adults in the same bilingual community. This finding is supported by the fact that our small study of the speech specifically directed by adults to Dewi showed the same patterns. This close relationship between the ML in the adult input and in child speech has not to our knowledge been previously demonstrated. Referring back to previous work we therefore find support for Eichler, Hager, and Müller's (2012) finding regarding the crucial role of the ML in child code-switching patterns. However, we have gone one step further in tracing the choice of the ML to its frequency in the input, thus providing support for a usage-based approach to bilingual acquisition. In line with the results from Yip and Matthews (2016) and Eichler, Hager, and Müller (2012), the results here suggest that children are code-switching because they are acquiring the patterns of speech produced in the input available to them. There is a striking similarity found here between the children's ML distribution and the ML distribution in the input to them and this input is provided not only by their parents but also by other adults in the community.

Interestingly, these results indicate that the age at which the children start reproducing adult code-switching patterns is much earlier than 3 years old, contrary to previous findings by Nicoladis and Genesee (1997). The material used in our study came from transcripts when the children were between the ages of $1 ; 9$ and 2;6. More research into different code-switching contexts is of course necessary in order to determine whether this behaviour of reflecting adults' code-switching input patterns at such a young age is the norm or an exception.

This study only focuses on one language pair (Welsh/English), and only data from communication between bilinguals has been analysed. Furthermore, the pattern of ML usage in this community is not found in all adult bilingual communities. It would be informative to study the 
code-switching acquisition pattern of children in other bilingual communities, which show a different distribution of the ML. For instance, the analysis of the ML of Spanish/English data from Miami shows variation between English ML and Spanish ML in the code-switching of the adult population (Parafita Couto et al. 2014). It would be fruitful to test the prediction that child ML distribution will mirror adult ML distribution on the Miami data. Comparing input relationships in contact situations where the predominant ML of either the adults or the children is in the process of change would also be fruitful, especially because ML changes have previously been linked to language attrition (cf. Myers-Scotton 2002, 251).

One consequence of the focus mentioned above on communication between bilinguals is that we have not been able to study how children acquire the ability to code-switch between utterances as a result of a change in interlocutors. All Welsh speakers in Wales need to address monolingual English-speaking interlocutors on some occasions, and hence to switch from Welsh to English when the situation demands it. This did not arise in the data we analysed because all speakers were bilingual. However, Deuchar and Quay (2000) were able to show that an English/Spanish developing bilingual accommodated in her language choice to that of her interlocutor as young as $1 ; 7$. It would be interesting to investigate the development of this language accommodation ability in a study of Welsh/English bilingual children addressing English monolinguals.

\section{Conclusion}

The results from this investigation into Welsh/English child code-switching indicate that the adult input plays an important part in influencing the patterns of the children's code-switching. The ML distribution for the codeswitching of adult and child populations surveyed was strikingly similar. The results from our investigation of adult speakers in the community (as in the Siarad corpus) and speakers on the CIG1 corpus recordings both indicate the same pattern of switching as that found in the child speech. Furthermore, our validity check on the main analysis of child speech in our second analysis yielded the same results. The main contribution of this investigation is that it provides a new angle for further study into child code-switching research, specifically looking at the effect of the adult input patterns on child code-switching. Where the motivations for child codeswitching have previously been tied to developmental stages and to dominance, further support is found here for an approach that suggests that child code-switching does not differ qualitatively from adult code-switching, at least if the choice of the Matrix Language is taken as the main criterion as in our study. The available evidence thus suggests that code-switching patterns in the same way as adult code-switching, and children acquire the patterns needed to code-switch with adult-like competence. 


\section{Appendix I}

\section{Gloss abbreviations}

$\begin{array}{ll}\text { 1S } & \text { 1st person singular } \\ \text { 3P } & \text { 3rd person plural } \\ \text { 3S } & \text { 3rd person singular } \\ \text { ADJ } & \text { adjective } \\ \text { ASP } & \text { aspect marker } \\ \text { CL } & \text { classifier } \\ \text { DEM } & \text { demonstrative } \\ \text { F } & \text { feminine } \\ \text { FUT } & \text { future/habitual present } \\ \text { M } & \text { masculine } \\ \text { NONFIN } & \text { non-finite verb } \\ \text { PL } & \text { plural } \\ \text { POSS } & \text { possessive } \\ \text { PREP } & \text { preposition } \\ \text { PRES } & \text { present } \\ \text { PRON } & \text { pronoun } \\ \text { PRT } & \text { particle } \\ \text { SFP } & \text { sentence final particle } \\ \text { SG } & \text { singular }\end{array}$

\section{Notes}

1 We use the terms "code-switching" and code or "language-mixing" interchangeably.

2 Information listed on the TalkBank website (https://childes.talkbank.org/access/ Celtic/Welsh/CIG1.html)

3 Many words coded as either Welsh or English are borrowings from English into Welsh. For further details of the transcription and language marking system see Deuchar, Webb-Davies, and Donnelly $(2018,35-42)$; and for our view on distinguishing code-switches from borrowings see Stammers and Deuchar (2012) and Deuchar, Webb-Davies, and Donnelly (2018) chapter 4.

4 Our data can be accessed at https://osf.io/gtj6v/

5 Bolded text indicates English language material. Underlined words in examples indicate material which could be Welsh or English.

6 Translations and glosses were added by the authors of the current paper.

7 We used the online version of Geiriadur Prifysgol Cymru see http://www.geiriadur.ac.uk/

8 For information regarding the transcribers' conventions for indicating uncertainty, see https://childes.talkbank.org/access/Celtic/Welsh/CIG1.html

9 The total refers to tokens rather than types; repeated utterances are included in the count.

\section{References}

Aldridge, M, R. Borsely, S. Clack, and G. Creunant. 1998. "The Acquisition of Noun Phrases in Welsh". In Language Acquisition: Knowledge Representation and Processing, edited by Antonella Sorace, Caroline B. Heycock, and Richard Shillcock. Edinburgh: University of Edinburgh Press. 
Baker, A. and B. van den Bogaerde. 2008. "Code-mixing in Signs and Words in Input to and Output from Children”. In Sign in Bilingualism: Language Development, Interaction, and Maintenance in Sign Language Contact Situations, edited by Carolina Plaza-Pust and Esperanza Morales-López, 1-28. Amsterdam: John Benjamins Publishing. doi:10.1075/sibil.38.04bak

Behrens, H. 2012. "Corpus Analysis of Child Language". In The Encyclopedia of Applied Linguistics, edited by C.A. Chapelle. Hoboken, NJ: Wiley. Doi:10.1002/9781405198431.wbeal0242

Blokzijl, J., M. Deuchar, and C. Parafita Couto. 2017. "Determiner Asymmetry in Mixed Nominal Constructions: The Role of Grammatical Factors in Data from Miami and Nicaragua". Languages 2 (4): 20. Doi:10.3390/languages2040020

Carter, D., M. Deuchar, P. Davies, and M.C. Parafita Couto. 2011. "A Systematic Comparison of Factors Affecting the Choice of Matrix Language in Three Bilingual Communities”. Journal of Language Contact 4: 1-31. Doi:10.1163/18 7740911X592808

Chomsky, N. 1980. Rules and Representations. Oxford: Blackwell.

Chomsky, N. 1986. Knowledge of Language: Its Nature, Origin and Use. New York; London: Praeger.

Comeau, L., F. Genesee, and L. Lapaquette. 2003. “The Modeling Hypothesis and Child Bilingual Codemixing”. International Journal of Bilingualism 2 (7): 113126. Doi:10.1177/13670069030070020101

Davies, P. and M. Deuchar. 2014. "Auxiliary Deletion in the Informal Speech of Welsh-English Bilinguals: A Change in Progress”. Lingua 143: 224-241. Doi:10.1016/j.lingua.2014.02.007

De Houwer, A. 1990. The Acquisition of Two Languages from Birth: A Case Study. Cambridge: Cambridge University Press.

Deuchar, M. 1999. “Are Function Words Non-Language-Specific in Early Bilingual Two-Word Utterances?” Bilingualism: Language and Cognition 2 (1): 23-34. Doi:10.1017/S1366728999000127

Deuchar, M. 2012. “Code-switching”. In The Encyclopedia of Applied Linguistics, edited by C. A. Chapelle, 675-664. New York: Wiley.

Deuchar, M. and A. Clark. 1996. "Early Bilingual Acquisition of the Voicing Contrast in English and Spanish". Journal of Phonetics 24 (3): 351-365. Doi:10.1006/jpho.1996.0019

Deuchar, M. and S. Quay. 2000. Bilingual Acquisition: Theoretical Implications of a Case Study. Oxford: OUP.

Deuchar, M. and M. Vihman. 2002. "Language Contact in Early Bilinguals". In Contact-Induced Language Change, edited by M.C. Jones and E. Esch, 267-281. The Hague: Mouton. Doi:10.1515/9783110892598.267

Deuchar, M. and M. Vihman. 2005. "A Radical Approach to Early Mixed Utterances”. International Journal of Bilingualism 9 (2): 137-157. Doi:10.117 7/13670069050090020201

Deuchar, M., P. Webb-Davies, and K. Donnelly. 2018. Building and Using the Siarad Corpus: Bilingual Conversations in Welsh and English. Vol. 18 of Studies in Corpus Linguistics. Amsterdam/Philadelphia: John Benjamins Publishing Company.

Döpke, S. 1992. One Parent, One Language: An Interactional Approach. Amsterdam: John Benjamins.

Eichler, N., M. Hager, and N. Müller. 2012. "Code-Switching within Determiner Phrases in Bilingual Children: French, Italian, Spanish and German”. Zeitschrift Für Französische Sprache Und Literatur 122 (3): 227-258. 


\section{Shannon Phillips and Margaret Deuchar}

Gardner-Chloros, P. 2009. Code-Switching. Cambridge: Cambridge University Press.

Gathercole, V.M. and E. Thomas. 2009. "Bilingual First-Language Development: Dominant Language Takeover, Threatened Minority Language Take-Up". Bilingualism: Language and Cognition 12 (2): 312 -237. Doi:10.1017/S136672 8909004015

Goodz, N. 1989. "Parental Language Mixing in Bilingual Families". Infant Mental Health Journal 10: 25-43. Doi:10.1002/1097-0355(198921)10:1<25:: AID-IMHJ2280100104>3.0.CO;2-R

Grimstad, M.B., T. Lohndal, and T.A. Åfarli. 2014. "Language Mixing and Exoskeletal Theory: A Case Study of Word-Internal Mixing in American Norwegian”. Nordlyd 41 (2): 213-237. Doi:10.7557/12.3413

Grosjean, F. 1989. "Neurolinguists, Beware! The Bilingual is not Two Monolinguals in One Person". Brain and Language 36 (1): 3-15. Doi:10.10 16/0093-934X(89)90048-5

Hebblethwaite, B. 2010. “Adverb Code-Switching Among Miami’s Haitian CreoleEnglish Second Generation”. Bilingualism: Language and Cognition 13 (4): 409-428.

Hoffmann, C. 1985. "Language Acquisition in Two Trilingual Children”. Journal of Multilingual and Multicultural Development 6 (6): 479-495. Doi:10.1080/01 434632.1985.9994222

Ihemere, K. 2016. "In Support of the Matrix Language Frame Model: Evidence from Igbo-English Intrasentential Code-Switching”. Language Matters 47 (1): 105-127. Doi:10.1080/10228195.2015.1110194

Joshi, A. 1985. "Processing of Sentences with Intrasentential Code-Switching". In Natural Language Parsing: Psychological Computational, and Theoretical Perspectives, edited by D. Dowty, L. Karttunen, and A. Zwicky. Cambridge: Cambridge University Press.

Khan, A.A. and A. Khalid. 2018. "Pashto-English Codeswitching: Testing the Morphosyntactic Constraints of the MLF Model”. Lingua 201: 78-91. Doi: 10.1016/j.lingua.2017.09.002

Köppe, R. 1997. Sprachentrennung im frühen bilingualen Erstspracherwerb Französisch, Deutsch. Germany: Narr.

Lanza, E. 1997a. Language Mixing in Infant Bilingualism: A Sociolinguistic Perspective. United Kingdom: Clarendon Press.

Lanza, E. 1997b. "Language Contact in Bilingual Two-year-olds and Codeswitching: Language Encounters of a Different Kind?*” The International Journal of Bilingualism 1 (2): 135-162. Doi:10.1177/136700699700100203

Langacker, R. 1987. Foundations of Cognitive Grammar. 2 vols. California: Stanford University Press.

MacWhinney, B. 1991. The CHILDES Project: Tools for Analyzing Talk. United Kingdom: Lawrence Erlbaum.

Myers-Scotton, C. 1993. Duelling Languages: Grammatical Structure in CodeSwitching. $1^{\text {st }}$ edition. Oxford: Clarendon Press.

Myers-Scotton, C. 2002. Contact Linguistics: Bilingual Encounters and Grammatical Outcomes. Oxford: Oxford University Press.

Newport, E, H. Gleitman, and L. Gleitman. 1977. "Mother I'd Rather Do It Myself: Some Effects and Noneffects of Maternal Speech Style". In Talking to Children: Language Input and Acquisition, edited by C. Snow and C. Ferguson. Cambridge: Cambridge University Press. 
Nicoladis, E., and F. Genesee. 1997. "The Role of Parental Input and Language Dominance in Bilingual Children's Code-Mixing”. In BUCLD 21 Proceedings, 422-432. Somerville, MA: Cascadilla.

Paradis, J., E. Nicoladis, and F. Genesee. 2000. "Early Emergence of Structural Constraints on Code-Mixing: Evidence from French-English Bilingual Children". Bilingualism: Language and Cognition 3 (3): 245-261.

Parafita Couto, M.C., P. Davies, D. Carter, and M. Deuchar. 2014. "Uniformity and Variability in the Choice of Matrix Language: A Tale of Two Communities". In Unraveling Bilingualism: A Cross-Disciplinary Perspective, edited by E. Thomas and I. Mennen, 111-140. Bristol: Multilingual Matters.

Patuto, M., M. Hager, L. A. Gil, N. Eichler, V. Jansen, A. Schmeißer, and N. Müller. 2014. "Child-External and -Internal Factors in Bilingual Code-switching: Spanish, Italian, French and German”. In Language Contact Around the Globe. Proceedings of the LCTG3 Conference, edited by A. Koll-Stobbe and S. Knospe. Bern: Peter Lang.

Petersen, J. 1988. "Word-Internal Code-Switching Constraints in Bilingual Child's Grammar”. Linguistics 26: 479-493.

Pfaff, C. 1979. "Constraints on Language Mixing: Intrasentential Code-Switching and Borrowing in Spanish/English”. Language 55 (2): 291-318.

Poplack, S. 1980. "Sometimes I'll Start a Sentence in Spanish Y TERMINO EN ESPAÑO". Linguistics 18: 581-618. Doi:10.1515/ling.1980.18.7-8.581

Stammers, J., and M. Deuchar. 2012. "Testing the Nonce Borrowing Hypothesis: Counter-Evidence from English-Origin Verbs in Welsh”. Bilingualism: Language and Cognition 15 (3): 630-643. Doi:10.1017/S1366728911000381

Vihman, M. 1985. "Language Differentiation by the Bilingual Infant". Journal of Child Language 12: 297-324. Doi:10.1017/S0305000900006450

Vihman, M. 1998. "A Developmental Perspective on Codeswitching: Conversations between a Pair of Bilingual Siblings". International Journal of Bilingualism 2 (1): 45-84. Doi:10.1177/136700699800200103

Yip, V. and S. Matthews. 2016. "Code-Mixing and Mixed Verbs in CantoneseEnglish Bilingual Children: Input and Innovation”. Languages 1 (4): 1-14. doi:10.3390/languages1010004

Yip, V. 2013. "Language Acquisition”. In The Psycholinguistics of Bilingualism, edited by F.L. Grosjean, 134. Hoboken: John Wiley \& Sons. 


\section{Part II}

\section{Language Practices and Policies in the Family}




\title{
4 Language Development, Discourse, and Politics
}

\section{Family Language Policy Foundations and Current Directions}

\author{
Kendall A. King and Xiao Lan \\ Curdt-Christiansen
}

\section{Introduction}

This chapter presents three recent research projects in the area of family language policy (FLP) and highlights how Elizabeth Lanza's research has been foundational in the development of each. We organize our chapter around what we conceptualize as three strands of FLP work, each of which was largely defined or shaped by Lanza's ground-breaking scholarship. Early work is exemplified by Lanza's classic and highly influential research ([1997] 2004) which examined language contact and development within Norwegian-English bilingual families. This first strand of research used close discourse analysis of everyday family life to reveal what we now consider implicit FLPs and to unpack language development and use among young children. This approach and the insights from this research are still evident in recent works, as illustrated by close examination of language practices and language socialization in Malay and Chinese homes in Singapore (Curdt-Christiansen 2013a, 2013b, 2016a, 2016b). That project is presented and described here.

A second strand, evident in more recent research and framed explicitly under the banner of "family language policy" (e.g., Lanza and Li Wei 2016; Lanza and Curdt-Christiansen 2018; Curdt-Christiansen and Lanza 2018; King and Lanza 2018; Lanza 2020a) includes detailed discourse analysis of identity, ideology, and agency in understanding home language practices. This work is characterized by the use of an anthropological and ethnographic lens, as shown in multiple current projects, including Chinese families in the UK (e.g., Curdt-Christiansen and LaMorgia 2018; CurdtChristiansen 2020), one of which is detailed here.

A third and final strand of FLP takes up the political dimensions of FLP, including how nationalistic or anti-immigrant discourses impact family language decisions as well as how such decisions within families are in fact political ones. Work in this strand is evident in Lanza's examination of media and immigrant families in Norway (Purkarthofer, Lanza, and Finstad 
Berg, forthcoming) as well as current work, reviewed here, which analyses how Latino families understand restrictive immigration and deportation policies in the US (King and Fluegel, in press). Taken together, this chapter provides an overview of the development of the field of FLP with a focus on Lanza's important contributions, while simultaneously showcasing new empirical research in each of these three strands.

\section{Implicit Language Policies Within Families (Strand One)}

Lanza's early research focused on language practices and implicit policies within families. In her highly influential research of bilingual Norwegian and American families, Lanza ([1997] 2004) demonstrated how caregivers used discourse strategies to socialize children into particular language practices that resulted in divergent pathways for language development. She identified five types of discourse strategies that parents use in response to children's non-target language use: minimal grasp, expressed guess, repetition, move-on, and code-switch. In parent-child interactions, when using the minimal grasp strategy, adults pretend not to understand the language chosen by the child; the expressed guess strategy is used by adults when posing yes/no questions in the target language and accepting simple confirmation as an answer; the repetition strategy entails that adults repeat children's utterances in the target language; the move-on strategy is employed by adults when indicating comprehension and acceptance of children's (non-target) language choice, so that a conversation continues without any implicit and explicit disruptions; and with code-switch, adults either switch over completely to the other language or use an intra-sentential change of language. Lanza argued that these strategies can be placed on a continuum "indicating their potential for making a bid for a monolingual or bilingual context once the child has opened negotiations for a bilingual context through mixing" $(2007,56)$.

Lanza's early work, while not framed explicitly as FLP research, provided theoretical and analytical frameworks for researchers to conduct systematic studies of language use, development, and socialization among families in varied contexts (King, Fogle, and Logan-Terry 2008). Gafaranga (2010), for example, studied members of the Rwandan community in Belgium in which a language shift from Kinyarwanda-French bilingualism to French monolingualism was taking place. He employed Lanza's discourse strategies to analyze interactions between children and adults in the community and identified a "medium request" strategy used by youths to "talk language shift into being" (2010, 241). In a similar vein, Smith-Christmas's studies $(2016,2018)$ of an English-Gaelic speaking family on the Isle of Skye (UK) demonstrated the varied discourse strategies that were used by family members in their negotiations of which language to use in their daily routines and the implications of 
those patterns for language development. Lomeu Gomes (2020) also used the analytical framework to examine the language practices and ideologies of a Brazilian-Norwegian family in Norway. This framework of close analysis of discourse strategies allows researchers to uncover how small, everyday interactional moves result in particular language competencies, often favoring the dominant language.

Taking up these same tools, Curdt-Christiansen (2013a) studied three Chinese-English-speaking families in Singapore; she found that the mothers used different strategies when helping their children with homework. Based on her analysis of the bilingual interactions between the mothers and their children, she argued that depending on the situation and norms of code-switching acceptance in the community, mothers tended to use discourse strategies ranging from conscious requests to unconscious accommodation. She found that the discourse strategy employed is closely related to the types of FLP used in these families. For instance, the mother in the family with highly organized FLP tended to use more Chinese and less English when providing input on the development of ideas; she did so by using decontextualized academic vocabularies in Chinese despite the fact that the homework was in English. In the family with relatively unreflective FLP, the mother tended to use the "move-on" strategy and unconsciously used English when asking for clarification. The third type of identified FLP was a laissez-faire policy; in that family, the mother seemed to pay little attention to her child's dominant use of the English language when doing his Chinese homework. While all three families desired "balanced" bilingual outcomes for their children, their discourse strategies did not align with their conscious efforts with respect to stated FLPs.

In what follows, we demonstrate how Lanza's framework remains relevant and productive in multilingual families in relation to FLP in the context of Singapore, where both English and varied mother tongues are used simultaneously in different domains. These linguistic practices are the result of a forceful top-down government language policy, which recognizes four official languages: English, Mandarin, Malay, and Tamil. These official languages are, however, not equally valued. While English enjoys a high prestige because it is the language of law, business, and public affairs as well as the medium of instruction in all schools across all subjects at all levels, the remaining three official languages are designated as mother tongues, which are recognized as repositories of culture and identity related to the country's three major ethnic groups - Chinese, Malay, and Indian (CurdtChristiansen 2016a).

The (unpublished) data shared here are drawn from a larger project (2007-2010) that explored language socialization in Singaporean families by examining social interactions in multilingual families of the three major ethnic groups. The project focused on how children (ages 3-7) acquire sociocultural beliefs and knowledge through participation in language-mediated 
interactions. Data from 18 families were collected over a two-year period through ethnographic observations and interviews. Regular visits (once every three or four weeks) were conducted, and 10-15 recorded interactions (5-30 minutes) were collected from each family. Here, we present data from two Malay and two Chinese families to show how individuals in these families make sense of the different languages they use in their everyday life.

Excerpt 1 is taken from the Zakri family, which consisted of Adena

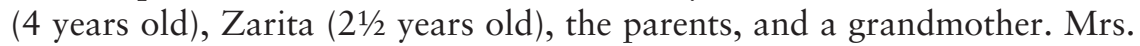
Zakri is an accountant of a large firm and Mr. Zakri a manager of the same firm. Because of their busy professional life, Adena and her little sister stay at the grandmother's house during the week and return to their parents' house on weekends. This shared child-rearing practice is widely accepted in Singapore. Adena's parents speak mostly English to her, and her grandmother speaks both English and Malay (Bahasa Melayu) to her.

Excerpt 1 is a dialogue between Adena and her grandmother on their way home from school. They are talking about the food Adena had in school.

Excerpt 1: What did you have for lunch? (October 5, 2009)

(Grandma: G; Adena: A; //-//: simultaneous utterance; bold type: codeswitching; English translation below the Malay original)

$1 \mathrm{G}: \quad$ Tengah hari makan apa?

What did you have for lunch?

2 A: //Maken..//

//Ate..//

$3 \mathrm{G}: \quad \mathrm{Nasi} / \mathrm{ke}$ ?

Ricel/?

4 A: Nasi//Nasi (nods her head)

Ricel/Rice.

5 G: Nasi dengan apa?

Rice with what?

6 A: Nasi. Dengan chicken

Rice with chicken

$7 \mathrm{G}$ : Kengtang?

Potatoes?

8 A: And kengtang.

And potatoes.

9 G: Then Lagi?

Then what else? 
10 A: Lagi ... soup

Some more soup.

$11 \mathrm{G}$ : Soup? Apa dia letak dalam soup dia?

Soup? What did she put in the soup?

$12 \mathrm{~A}$ : Chin chai [vegetable in Chinese]

Chin chai

13 G: Huh? Chin Chai? Apa chin chai?

Hub? Chin Chai? What is Chin Chai?

$14 \mathrm{~A}$ : Chin chai is a vegetable ... It's from Chinese

15 G: Bukan chin chai. Chye sim.

Not Chin Chai. Chye sim

$16 \mathrm{~A}$ : This one is Chinese.

$17 \mathrm{G}$ : Cina punya sayur, chye sim!

A Chinese vegetable, chye sim!

$18 \mathrm{~A}$ : Chin chai [insists on using Chinese]

19 G: Bukan chin chai, CHYE SIM. Abih sedap tak nasi dia?

Not Chin Chai, CHYE SIM. So, was the rice delicious?

In this series of questions and answers, the grandmother used several strategies, including move-on, adult repetition, and code-switches. For instance, in line 6, when Adena answered: "Nasi. Dengan chicken" (rice with chicken) to the question "Nasi dengan apa?" (rice with what?), the grandmother used a move-on strategy to continue with the dialogue. Consequently, the conversation carried on with Adena using both English and Malay in her utterances (L8) despite her grandmother's consistent use of Malay. In line 9, Grandma seemed to follow the cue by Adena; she also added an English conjunction then in her utterance "Then Lagi?" This move is another type of move-on strategy in which adults accept children's language behavior and carry on with the conversation (L9 and L11). The conversation then shifted to metalinguistic talk of what Chin Chia is in L13. Interestingly, in lines 14-17, Grandma used Malay to request a clarification about Chin Chia when Adena responded to the request in English. This move represents the minimal grasp strategy. However, Adena refused to repeat the utterance in Malay. Despite her intention to correct Adena in line 9, "Bukan chin chai, CHYE SIM" (Not Chin Chai, CHYE SIM), grandmother chose to desist by using a move-on strategy to continue the communication undisrupted by asking "Abih sedap tak nasi dia?" (So was the rice delicious?). The interactional moves between Grandma and Adena show not only the implicit discourse strategies for negotiation of language 
choice, but also the subtle influence of macro language policy on family language practices.

Excerpt 2 consists of a dialogue from the Yahaya family. Mrs. Yahaya is a school teacher and her husband a lawyer. Ali (4 years old) goes to a daycare during the week. The parents speak English and Malay. Although, like all parents in the study, they want Ali to become a "balanced" bilingual speaker, they tend to correct Ali when he uses Malay, as illustrated in the dialogue between Ali and his mother. In the short excerpt, Ali talks about seeing a bird in the schoolyard.

Excerpt 2: Kejar a bird (April 4, 2008) (Mrs. Y: M; Ali: A)

$1 \mathrm{M}$ : What did you do to the bird?

2 A: I kejar him. I chase him.

$3 \mathrm{M}$ : What did you do?

4 A: I chase him.

In this conversation, Ali inserted kejar (chase) into his English rejoinder to his mum's question. The mother was not particularly happy with the answer; she used a minimal grasp strategy through a direct request asking Ali to self-correct his utterance. The implicit socialization practice has a clear ideological overtone, directly related to the government's language policy in which English is given space to develop in both public and home domains.

Similar discourse strategies have also been observed in Singaporean Chinese families when parents intend to develop their children's Chinese language. Excerpt 3 is a typical example from the Goh family, which consisted of Mr. Goh (businessman), Mrs. Goh (housewife), Feng (31/2 years old) and his big sister Ming (5 years old). Mr. and Mrs. Goh speak both English and Mandarin at home. Feng and Ming, however, prefer to speak English. Concerned about the children's Chinese language development, both Mr. and Mrs. Goh try to use more Mandarin in their daily family talks. Excerpt 3 is a dialogue between Feng and his mother when they were reading a picture book together.

Excerpt 3: Butterfly 叫什么? (October 20, 2009) (Feng: F; Mrs. Goh: M)

$1 \mathrm{~F}$ : Butterfly [points to a picture of a butterfly in the book].

$2 \mathrm{M}$ : What butterfly? Butterfly 叫什么? What butterfly? What is butterfly called? [in Chinese] 


\section{$3 \mathrm{~F}:$ 蝴蝶}

\section{Butterfly [pronounced: hudie]}

$4 \mathrm{M}$ : 好

Good.

It is noticeable in this dialogue that children's "default" language is English, illustrating the effect of the official bilingual policy in recent decades (CurdtChristiansen 2016a, 2016b). Some parents are aware of this slide toward English and have begun to give more explicit attention to children's language behavior. For instance, in Excerpt 3, Feng's mother used a direct request to elicit the Chinese word for "butterfly." In Excerpt 2, we see that parents tend to use more direct and expressed discourse strategies in their attempt to socialize their children into classroom-like practices.

Excerpt 4 provides another example of elicitation. The Teo family has five members, including two grandparents (retired), Mr. Teo (civil servant), Mrs. Teo (school teacher), and Damien (31/2 years old). Mr. and Mrs. Teo speak Mandarin with the grandparents but mostly English to Damien. Damien speaks mostly English to both his grandparents and parents, but Chinese words are occasionally inserted into his utterances.

Excerpt 4: What is lemon called in Chinese? (December 12, 2008)

(Mrs. Teo: M; Damien: D)

$1 \mathrm{M}$ : What is lemon called in Chinese?

2 D: I don't know.

$3 \mathrm{M}$ : What color is lemon?

$4 \mathrm{D}$ : 黄色。 Yellow.

$5 \mathrm{M}$ : lemon is 柠檬 Lemon is ningmeng

6 D: 柠檬.

Ningmeng.

Our observations of family talk reveals that parental discourse strategies tend to follow the widely documented teaching practices of a second-language or foreign-language classrooms in the form of Initiation-Response-Evaluation sequences (Sinclair and Coulthard 1975) as parents often ask direct questions seeking clarifications, requesting comprehension checks or asking for repetitions of vocabulary. In Excerpt 4, the mother asked direct questions in lines 1 and 3, and explicitly taught Damien the word lemon in Chinese in line 5. Although this approach does not match Lanza's parental established 
discourse strategies precisely, it is similar to minimal grasp or expressed guess strategies. In a recent study, Abreu Fernandez (2019) reported similar language practices in Russian-Swedish speaking families in Sweden. Using the term "language workout," she contends that this socialization register provides vocabulary input that helps scaffold children's language use.

Overall, these data suggest that in the Singaporean context parents and grandparents are quite conscious about and explicit in correcting or redirecting children's linguistic behavior, whereas in Lanza's work ([1997] 2004), parents tend to be more implicit or indirect in their socialization practices.

These rather mundane, everyday bilingual conversations illustrate that discourse strategies used by adults have degrees of consciousness and explicitness that vary contextually. In this respect, Singaporean parents/grandparents might intentionally socialize children into separate language use in Malay, English, or Chinese, but the conversations provide limited communicative practice for children to use the languages in a natural manner. Family language policy is thus established over time and based on the interactional styles co-constructed by adults and children. Lanza ([1997] 2004) emphasized that the degrees of implicitness of language socialization in families potentially contribute to the language outcomes.

\section{Identity, Ideology, and Agency in Family Language Policy (Strand Two)}

A second strand of research, evident in more recent work and framed explicitly under the banner of "family language policy" (e.g., Lanza and Curdt-Christiansen 2018; Lanza and Li Wei 2016; Lanza and King 2018; Lanza 2020b; Lanza and Lexander 2019; Lanza and Lomeu Gomes, 2020), includes a detailed analysis of identity, ideology, and agency to better understand home language practices and child language use patterns and development. This line of scholarship is frequently characterized by the use of an anthropological and ethnographic lens and is evident in multiple projects, including bilingual families in the US (King, Fogle, and Logan-Terry 2008), bilingual families in Singapore (CurdtChristiansen 2016a, 2016b), and Korean-English families in the US (Kang 2015), among others. One recent project (Curdt-Christiansen, ongoing) investigates FLP at multiple levels - national, community, and individual families within the UK. At the national level, a survey examines how mobility and the on-going sociopolitical changes influence FLP. At the community level, three communities (Chinese, Polish, and Somali) are studied to make visible the historical trajectories of cultural and linguistic development in relation to migration in diasporic communities. At the individual family level, the project examines how socioeconomic 
pressure, sociopolitical context, public education demands, and linguistic forces, as well as family structure shape the formation of FLP. In what follows, we present a case of one Chinese family from the study, examining their language practices and parental ideology by studying their digital and media practices.

The Liu family moved to England about ten years ago when the parents came to study as international students. They have two girls; the older, Jiejie (15 years old), was born in China and the younger, Meimei (8 years old), in the UK. Learning two languages, English and Chinese, is described by the parents as a smooth and happy journey for Jiejie but less so for Meimei. Before Meimei was born, the family language was predominantly Chinese, and Jiejie reportedly sailed through schools in the UK with excellent academic records. The family language practices continued in Chinese until Meimei was sent to a nursery; it was then that the parents began worrying about Meimei's communication with the adults and children in the nursery. The family language policy changed, as Mrs. Liu explained:

\begin{tabular}{|c|c|}
\hline $\begin{array}{l}\text { 我们非常担心妹妹在幼儿园的交 } \\
\text { 流和沟通问题。特别是老师说 } \\
\text { 妹妹很少说话, 但是她在家里 } \\
\text { 话很多, 不停地说。我们好担 } \\
\text { 心, 这肯定是语言的问题。我 } \\
\text { 们决定多说英语, 后来, 英语 } \\
\text { 越来越多, 那妹妹的中文就不 } \\
\text { 是很理想。她说出来的中文都 } \\
\text { 是外国腔。 }\end{array}$ & $\begin{array}{l}\text { We became very worried about Meimei's } \\
\text { communication skills at the nursery. } \\
\text { Especially when the teacher said that Meimei } \\
\text { hardly spoke at all. But she was very bubbly } \\
\text { at home, always talking non-stop. We } \\
\text { were so concerned. (To us) this was clearly } \\
\text { a language issue. That's why we decided } \\
\text { to change our home language to English. } \\
\text { Then, later on, English was used more and } \\
\text { more; then Meimei's Chinese became less } \\
\text { developed. Now she speaks Chinese like a } \\
\text { foreigner. }\end{array}$ \\
\hline
\end{tabular}

Interview with Mrs. Liu (June 20, 2018)

In this interview, Mrs. Liu recalled and reflected on the critical moment of decision-making when changing her family language policy. Her concerns appear to be widespread, as existing literature suggests that many parents worry about their children's mainstream language development in the host country (Canagarajah 2008; Curdt-Christiansen and LaMorgia 2018; Song 2019). As Spolsky (2012) pointed out, many immigrant parents have to deal with the social realities of public educational demands on the one hand and the desire to maintain family language practices on the other. With this sort of on-going linguistic competition, minority languages are often defeated because they lack public support, educational provisions, and community facilities. In the case of the Liu family, Meimei's Chinese language skills have decreased over the years, as illustrated by the social media communications between her and her family. 
Excerpt 5: Mummy, I miss you (June 8, 2018)

Fri 8 Jun, 16:07

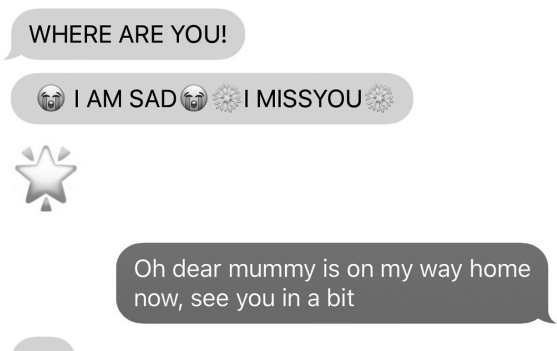

$\mathrm{Ok}$

The social media practices in the family between Meimei and her mother are shown in Excerpt 5, which provides a snapshot of the family's (largely English) language practices. Meimei used capital letters and different emojis when writing to her mum. The texts reflect a typical contemporary social media practice where individuals use multimodality to intensify or lighten up communications that convey their emotions, intentions, frustrations, and appreciations. In the short exchanges, Meimei used (for) (cry face) twice to show how sad she was without her mother.

Excerpt 6: Morning, Meimei (June 19, 2018)

Tue 19 Jun, 08:28

妈

蔵藏 早上好:

Have a nice day love $x x x$

ok

Tue 19 Jun, 21:42

Occasionally, Mrs. Liu also used Chinese in their communications, but the Chinese texts were often immediately followed by English texts, such as the two lines in Excerpt 6. Mum started the conversation by using Chinese to greet Meimei in the morning (when she was away from home), then followed up with the English text: Have a nice day love $x x x$. This communication style was a conscious reflection on the family's language use in recent years, after Mrs. and Mr. Liu had realized that Meimei's Chinese language development had more or less stopped. Although consciously providing opportunities for Meimei to relearn Chinese by sending her to a Chinese school, the communication pattern in the family was difficult to rectify. Mrs. Liu acknowledged that it is difficult for Meimei to communicate with 
the grandparents without the parents' language prompts and support. In the next excerpt, we illustrate a WeChat exchange between Meimei and her grandfather. Meimei recorded her violin rehearsal for grandpa, which she sent together with a voice message asking what grandpa thought about her performance. While the recording was in Chinese, a background coaching from her mother could be clearly heard.

Excerpt 7: Do you love me, grandpa? (January 23, 2019)

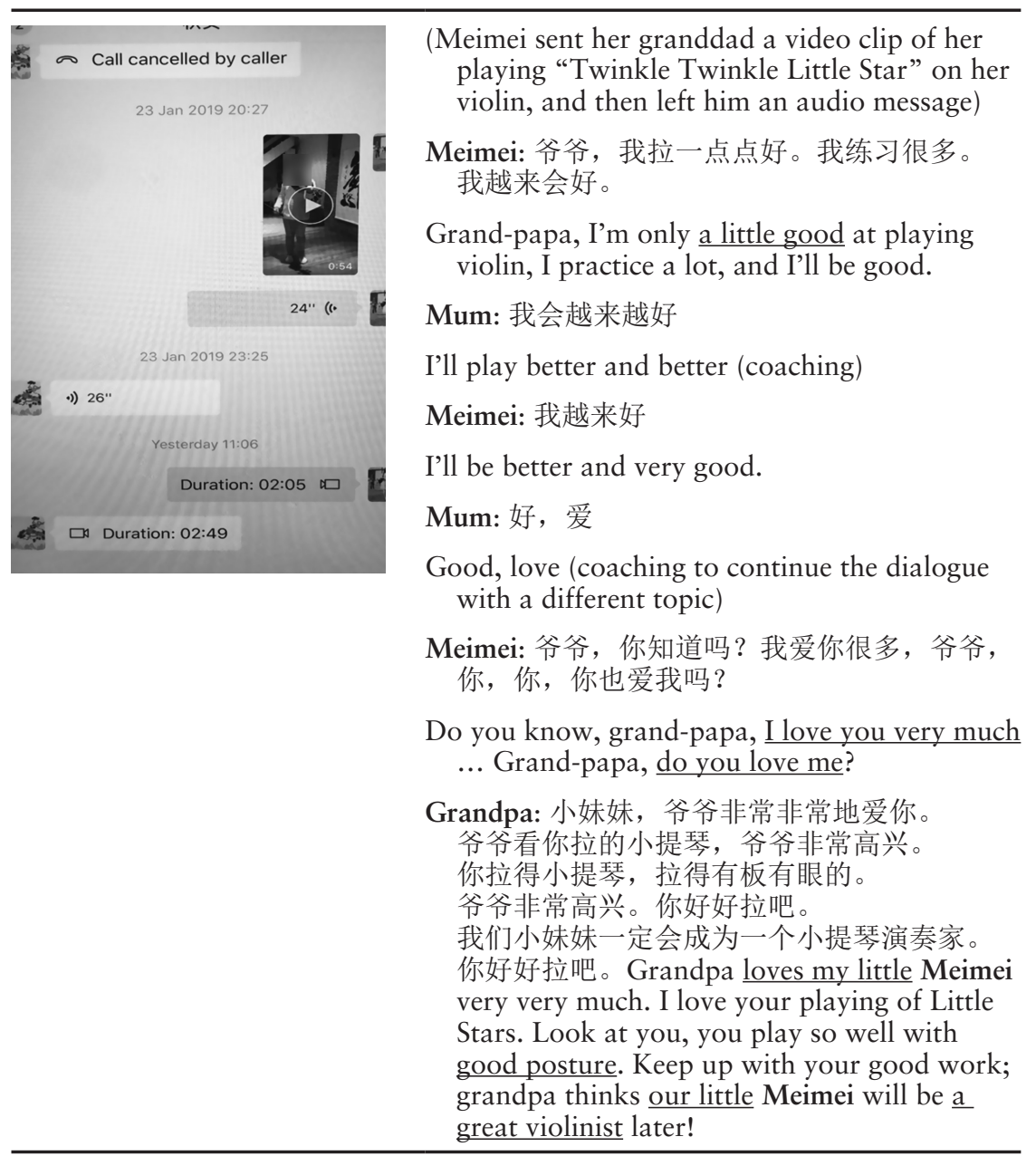

This WeChat exchange illustrated one encounter within a language revitalization initiative in the family. From a heritage language development perspective, this FLP decision can be viewed as a deliberate measure and conscious move to increase Meimei's Chinese language use in real language 
communication. Meanwhile, it is also an opportunity to enforce the emotional ties between Meimei and her grandfather. From an FLP perspective (King, Fogle, and Logan-Terry 2008; King and Fogle 2006), the coaching from the mother goes beyond her parenting intentions as a good mother to include socializing Meimei into using Chinese, demonstrating different functions of Chinese (communication tool with grandparents), and negotiating culturally influenced behaviors and social identities for using expressions. For example, "I am a little good" indicates a modest behavior, "I practice a lot" shows her diligence, and "I love you very much" demonstrates her affection for grandpa. The use of "I" in Meimei's utterances illustrates a strong agency and identity as she "relearns" Chinese and is coached by her mother. The use of "my, our little" from grandpa reinforces their mutual affection, which is further strengthened when grandpa predicts that Meimei will be "a great violinist later."

These data suggest the ways that family language policy in transnational families is context-specific and largely depends on the parents' expectations and aspirations for their children's language and educational development. At the same time, child agency is also evident as a factor influencing family language practices as children negotiate their identity through language use. FLP depends on the sociopolitical environment for supporting bilingual and multilingual development. Very often, parental language aspirations encounter challenges when they collide with societal ideologies that place little value on minority languages. FLP, thus, is not only driven by various language ideologies within families and in society, but it also embodies ideologies in linguistic practices, as evidenced in Meimei's case and in studies by other scholars (Curdt-Christiansen and Lanza 2018; King and Lanza 2018; Lanza and Curdt-Christiansen 2018). FLP has important implications for policymakers at macro (national), meso (educational), and micro (family) levels as it not only concerns explicit and deliberate language planning (King, Fogle, and Logan-Terry 2008) but also meaning-making and language-mediated experiences in families.

\section{Political Dimensions of Family Language Policy (Strand Three)}

A third strand of FLP takes up the political and media dimensions of FLP, including how nationalistic or anti-immigrant discourses impact family language decisions as well as how language decisions and practices within families are in fact political ones. Work in this strand is evident in Lanza and her colleagues' examination of how families and FLP are framed by media and state institutions and how the public is discursively integrated into (or kept out of) the family space.

Exemplary of this work is Purkarthofer, Lanza, and Finstad Berg's (forthcoming) investigation of six years of media coverage and public understandings of Barnevernet, the Norwegian Child Welfare Services Agency. 
Norwegian law strongly favors the rights of the child (relative to those of the family or community), in some instances resulting in the temporary or permanent removal of the child from the family, ostensibly to protect the child from harm. Statistics suggest that immigrant, foreign-born, and minority-language children are disproportionately likely to be removed from their homes by Barnevernet, leading to questions - and public media coverage - concerning the role of the state, the nature of the family, and what constitutes good parenting. Purkarthofer, Lanza, and Finstad Berg (forthcoming) illustrate how media discourses about Barnevernet provide a means to understand multilingual participation in society. In their analysis of media coverage and parent interviews, Purkarthofer, Lanza, and Finstad Berg (forthcoming) find that participants in their study attempt to present their family experiences as "normal." In doing so, they are not only answering to Norwegian expectations about their private spaces but also taking on and reacting to particular public expectations and constructions of family spaces as "good," "appropriate," and ultimately as "safe."

Lanza and her colleagues report that language issues are a major component of the expectations around parenting. In Norway, as in many other countries, politicians actively promote their visions of (more often than not monolingual) societies and parents. Parents in Norway who do not meet these expectations are at risk (or led to believe they are at risk) of losing custody of their children. Their findings have implications for our understanding of how families are defined in Norway and, more broadly, for the study of family language policies in multilingual families (Lanza and Li Wei 2016). The research of Lanza and her colleagues here also points to the importance and value of critical examination of the media - both for what such work can tell us about how "good" or "bad" families are conceived (driven at least in part by the FLPs therein) and the impact of media and social media in shaping FLP. This is an important direction, given the everincreasing access to and influence of all media and the ever-more blurred lines between "mainstream" and "social" media (Shearer and Gottfried 2017). While social media has a profound impact on how we understand and make sense of the world around us more broadly, for parents (and, evidently, mothers in particular) social media have been found to be a powerful source of information and connection and, concomitantly, a source for the understanding of what it means to be a good parent (Duggan et al. 2015).

Perhaps universally, the most basic and crucial component of being a good parent is keeping one's child safe. For many immigrant-background parents in the US, policies and public and media discourses around immigration have direct implications for safety and, indirectly, for family language policy. This is particularly the case for Spanish-speakers, the language spoken by the majority of immigrants in the US (with residents, migrants, and refugees hailing from more than a dozen different Spanish-speaking countries). Despite the long history of Spanish in the US and a large number of speakers (roughly 53 million), Spanish, in many areas of the country, 
is politicized and can be both stigmatized and stigmatizing (Carter 2018; Machado 2019).

The politicization of Spanish intensified under the Trump administration as the deportation of immigrants emerged as a central component of his campaign to "make America great again." In the first eight months of the Trump administration, immigration arrests in the interior of the US, particularly of immigrants without a criminal background, increased by $42 \%$ over the same period the year prior (Lopez, Gonzalez-Barrera, and Krogstad 2018). In subsequent years, deportation numbers have fluctuated (e.g., 288,000 new deportations in the fiscal year ending September 30, 2018) and 143,000 arrests in the 2019 fiscal year, marking the lowest number of arrests to date under the Trump administration. This is a relatively low number of deportations in light of Trump's announcement on Twitter in June 2019 that Immigration and Customs Enforcement (ICE) was preparing to "begin the process of removing the millions of illegal aliens who have illicitly found their way into the United States." While that operation proved largely unsuccessful, these twists and turns in policy and practice have been, to say the least, unnerving for many of the estimated 12 million undocumented individuals who lack legal status (and have no path for attaining it) and currently reside in the US.

Crucial here is the fact that official criteria for questioning, detainment, and potential deportation are largely opaque (Funk 2019). Officially, US ICE is barred from racial profiling and maintains that agents receive training to that effect every six months (Surana 2018). There is, however, ample evidence that race and language in fact render individuals suspect throughout the country. Indeed, US and international media regularly report instances in which individuals were stopped, questioned, and detained by ICE or border officials for speaking a language other than English, most often Spanish. Prominent examples from the last few years include two women (US citizens) who were detained for chatting in Spanish while waiting in a Montana convenience store line (Associated Press 2018). The women have since filed a lawsuit in federal district court to order the US Customs and Border Protection not to stop or detain anyone "on the basis of race, accent and/or speaking Spanish," unless those characteristics are tied to a specific and reliable suspect description (BBC 2018; Wofsy and Borgmann 2019).

Speaking Spanish is routinely portrayed on social media as putting one at risk of being deported, detained, or worse. Spanish-language newspapers regularly run headlines noting, for instance, that "Speaking Spanish in U.S. is a Deportation Risk" (20minutos 2018; La Vanguardia 2018). Widely circulating videos, such as that of a New York City lawyer, Aaron Schlossberg, who denounces restaurant employees for speaking Spanish to each other and shouts that his "next call is to ICE" (BBC 2018; Robbins 2018), effectively create an atmosphere in which Spanish-speakers are positioned as undocumented and at risk of deportation or worse (Carroll 2017). 
Perhaps the most tragic example is that of the Spanish-speakers and immigrant families that were intentionally targeted by the 2019 mass shooter in El Paso, Texas, who killed 22 people and injured 24 others. While this incident was extreme and horrific, it is not unusual in its target: anti-Hispanic incidents have made up around half of all reported ethnic-bias hate crimes since 2004, according to federal data (Fermoso 2018).

Survey data suggest these events and their intense social media coverage affect the lives and experiences of Spanish-speakers in the US. About 40\% of Latinos report feeling discriminated against because of their Latino background (Lopez, Gonzalez-Barrera, and Krogstad 2018). Pew data indicate that the majority of Latinos in the US $(55 \%)$, regardless of legal status, say they worry "a lot" or "some" that they, a family member or a close friend could be deported, up from 47\% who said the same in 2017 (Lopez, Gonzalez-Barrera, and Krogstad 2018). Further, fully two-thirds (66\%) of immigrant Latinos report they worry about deportation, compared with $43 \%$ of those who were born in the US. The share rises to $78 \%$ among those who are likely to be unauthorized (undocumented) immigrants. Language factors come in here as well: Latinos who speak Spanish as their primary language are more likely to worry about deportation than those who speak English as their primary language.

The study of FLP, as Lanza suggests, must take into account these events and their coverage on social media; concomitantly, analysis of social media can help us understand the tensions parents face in establishing their own family language policies. As summarized below, analysis of media geared for Spanish-speaking, immigrant-background families suggests gaps and contradictions with respect to what it means to be a "good parent" (King and Fogle 2006) within a highly politicized context in which Spanish is associated with undocumented legal status (King, in progress).

Analysis of Spanish-language parenting blogs, immigrant support agencies' websites, and social media sites for Spanish-speaking US-based families suggests that safety and preparing for the possibility of detainment and/or deportation are important topics for many Spanish-speaking parents. For instance, many non-profit organizations host sites, materials, and discussion platforms that contain guides, pamphlets, and printable information cards for parents concerned about family separation and potential deportation. These platforms and materials provide tips for talking with children about raids, advice on what to do if ICE comes to the door, and guidance on how to prepare adult and child family members emotionally and logistically for separation. These Spanish-language materials, particularly those published by public service and advocacy organizations, underline the ways in which deportation is a pressing worry and practical concern for many undocumented parents (e.g., Women's Refugee Commission 2019; Southern Poverty Law Center 2020; 20minutos 2018). In these Spanishlanguage materials, Spanish is assumed to be the routine language of communication within the family. 
In contrast, commercially driven social media platforms, blogs, and advice sites targeting Spanish-speaking parents in the US feature a sunnier view of life in the US. These ad-based platforms promote Spanish as a cognitive, academic, social, and occupational advantage for children (e.g., My Latina Table 2020; BabyCenter 2020; Familias 2020). Maintaining and developing Spanish, in these media, is crucial for keeping children connected with their heritage but also for allowing them to cash in on the "bilingual advantage." Such sites, blogs, and discussion forums delve into the "whys" and "hows" of bilingual parenting but make no mention of the political context of Spanish in the US. Many, for instance, assume that international travel is a possible means to promote child language skills and concomitantly ignore the well documented, widely circulating cases of linguistic profiling and anti-Latino discrimination.

In turn, Spanish-language political blogs or opinion pieces frame speaking Spanish publicly as an act of defiance and as a right or even an obligation. These pieces have titles such as "Why we should speak Spanish in public with pride and not be shamed" and exhort readers to do so (Univision 2017). Readers in such pieces are told, for instance, that "[we] should feel proud of our culture and our roots. We cannot have our identity stolen from us, nor speak our mother tongue in hiding" (Barahona 2018). Here, speaking Spanish is positioned as a badge of pride and possibly of courage as well.

In light of the above, for many Spanish-speaking immigrant-background families, and for undocumented parents, in particular, being a "good parent" (King and Fogle 2006) presents some profound dilemmas with respect to language. On the one hand, using Spanish with one's child, particularly in public, carries risks of detainment, harassment, or even violence. On the other hand, Spanish-English bilingualism provides important advantages and raising Spanish-speaking children is both a legal right and a moral obligation; within this discourse, "good parents" work hard to ensure their children develop and maintain their Spanish-language skills. This presents an unrecognized no-win, double-bind for many immigrant-background parents in which there are two idealized (and incompatible) images: the parent who cultivates private and public use of Spanish to give her child a bilingual edge, and the parent who protects her child (and family) from separation, discrimination, and danger. Current research (King, in progress) is examining how parents make sense of this conflict through analysis of parental narratives of language choice.

For researchers of family language policy, analysis of media and parents' understandings of their highly mediated worlds is a significant advance and new direction. With current research suggesting that US adults spend on average 11 hours per day consuming media (Fottrell 2018), the inclusion of this context is increasingly crucial for understanding FLP. In this respect, Lanza and her colleagues' analysis of Barnevernet in Norway was an important insight and advance in the field. 


\section{Conclusion}

This chapter presented three projects in the area of family language policy and highlighted how Elizabeth Lanza's research has been foundational in the development of each. This chapter has underlined the important contributions to the field made by Lanza and her collaborators at the outset and into the present. Her work over the last three decades has advanced our understanding of how languages are learned within families, how families create and define themselves through language, and how the politics of language can challenge families.

Questions of FLP seem all the more crucial in light of the myriad social, economic, and psycho-emotional stressors brought on by the COVID-19 pandemic. Worldwide, quarantines and lockdowns have centralized the family unit but simultaneously put it under huge stress. Concomitantly, technological connections with physically remote family members have become more routine, potentially offering new opportunities to support and grow minoritized languages in the home. Of course, the pandemic has also spotlighted, if not directly exacerbated, existing linguistic, economic, and social inequalities, including uneven access to educational and other state services (e.g., Sugarman and Lazarín 2020). Understanding how - and with what languages and language varieties - families are navigating this new landscape is an important, albeit it technologically and methodologically challenging, new research area in the years ahead.

Lastly, on a personal level, we also wish to recognize and thank Liz for being a supportive and collaborative colleague in our own academic development over the years. Her research has long centered on minority-language communities and the families within them; at the same time, she has built an active, strong, and diverse community of scholars around her as her academic legacy.

\section{Acknowledgment}

Part of the work in this paper is sponsored by the Economic and Social Research Council (ESRC) of Great Britain (ES/N019105/1).

\section{References}

20minutos. May 23, 2018. "Hablar español en Estados Unidos, un deporte de riesgo.” 20minutos. Accessed January 9, 2020. https://www.20minutos.es/notic ia/3347788/0/hablar-espanol-eeuu-riesgo/

Abreu Fernandes, O. 2019. "Language workout in Bilingual Mother-child Interaction: A Case Study of Heritage Language Practices in Russian-Swedish Family Talk." Journal of Pragmatics 140: 88-99.

Associated Press. May 26, 2018. "Why Immigrants in the U.S. Avoid Speaking Their Native Language.” Herald Tribune. Accessed January 10, 2020. https://www.her 
aldtribune.com/zz/news/20180526/why-immigrants-in-us-avoid-speaking-theirnative-language

BabyCenter. 2020. "Estrategias para criar ninos bilingues.” BabyCenter. Accessed January 10, 2020. https://espanol.babycenter.com/a2500013/estrategias-para-cri ar-ni\% C3\%B1os-biling\%C3\%BCes

Barahona, D. May 24, 2018. "Should We be Afraid of Speaking Spanish in Public?" La Noticia. Accessed January 10, 2020. http://www.lanoticia.com/afraid-ofspeaking-spanish-public/

BBC. May 22, 2018. "Why Spanish Speakers in US Are Getting into Trouble." BBC. Accessed January 9, 2020. https://www.bbc.com/news/world-us-canada-44201444

Canagarajah, A.S. 2008. "Language Shift and the Family: Questions from the Sri Lankan Tamil Diaspora.” Journal of Sociolinguistics 12 (2): 143-176.

Carroll, R. March 5, 2017. “America's Millions of Mexicans without Documents Live in Fear of Deportation." The Guardian. Accessed January 10, 2020. https:// www.theguardian.com/us-news/2017/mar/05/deportation-fear-mexicans-los-an geles

Carter, P.M. 2018. "Spanish in US Language Policy and Politics.” In The Routledge Handbook of Spanish as a Heritage Language, edited by K. Potowski, 36-52. New York: Routledge.

Curdt-Christiansen, X.L. 2013a. "Negotiating Family Language Policy: Doing Homework." In Successful Family Language Policy: Parents, Children and Educators in Interaction. Series Multilingual Education 7, edited by M. Schwartz and A. Verschik, 277-295. Dordrecht, Netherlands: Springer. doi:10.1007/978-94-007-7753-8_10.

Curdt-Christiansen, X.L. 2013b. “潜移默化 - Implicit Learning and Imperceptible Influence: Syncretic Literacy of Multilingual Chinese Children." Journal of Early Childhood Literacy 13 (3): 345-367. doi:10.1177/1468798412455819

Curdt-Christiansen, X.L. 2016a. “Conflicting Language Ideologies and Contradictory Language Practices in Singaporean Bilingual Families.” Journal of Multilingual and Multicultural Development 37 (7): 694-709. http://www.tandfonline.com/ doi/full/10.1080/01434632.2015.1127926

Curdt-Christiansen, X.L. 2016b. "Family Language Policy in the Chinese Community in Singapore: A Question of Balance?" In Multilingualism in the Chinese Diaspora Worldwide, edited by Li Wei, 255-275. London and New York: Routledge.

Curdt-Christiansen, X.L. 2020. "Educating Migrant Children in the UK: Language and Educational Practices in Home and School Environments." International Multilingual Research Journal 14 (2): 163-180. doi:10.1080/19313152.2020.1 732524

Curdt-Christiansen, X.L. and F. LaMorgia. 2018. “Managing Heritage Language Development: Opportunities and Challenges for Chinese, Italian and Urdu Speaking Families in the UK." Multilingua: Journal of Cross-Cultural and Interlanguage Communication 37 (2): 177-210. doi: 10.1515/multi-2017-0019

Curdt-Christiansen, X.L. and E. Lanza, eds. 2018. "Special Issue: Language Management in Multilingual Family: Efforts, Measures and Choices." Multilingua: Journal of Cross-Cultural and Interlanguage Communication 37 (2): 123-130. doi:10.1515/multi-2017-0132

Duggan, M., A. Lenhart, C. Lampe, and N.B. Ellison. 2015. "Parents and Social Media.” Pew Research Center. Accessed January 16, 2020. https://www.pew research.org/internet/2015/07/16/parents-and-social-media/ 
Familias. 2020. "Por favor, ¡habla en espanol a tus hijos!” Familias. Accessed January 10, 2020. https://www.familias.com/por-favor-habla-en-espanol-a-tushijos/

Fermoso, J. May 22, 2018. "Why Speaking Spanish is Becoming Dangerous in America." The Guardian. Accessed January 10, 2020. https://www.theguardian. com/us-news/2018/may/22/speaking-spanish-dangerous-america-aaron-schlos sberg-ice

Fottrell, Q. 2018. "People Spend Most of Their Waking Hours Staring at Screens." Accessed January 16, 2020. https://www.marketwatch.com/story/people-arespending-most-of-their-waking-hours-staring-at-screens-2018-08-01

Funk, M. October 2, 2019. "How ICE Picks Its Target in the Surveillance Age." The New York Times. Accessed January 10, 2020. https://www.nytimes.com/2019/1 0/02/magazine/ice-surveillance-deportation.html

Gafaranga, J. 2010. "Medium Request: Talking Language Shift into Being." Language in Society 39 (2): 241-270.

Kang, H. 2015. "Korean Families in America: Their Family Language Policies and Home-language Maintenance.” Bilingual Research Journal 38 (3): 275-291.

King, K.A. and L. Fogle. 2006. "Bilingual Parenting as Good Parenting: Parents' Perspectives on Family Language Policy for Additive Bilingualism." International Journal of Bilingual Education and Bilingualism 9 (6): 695-712.

King, K.A., L. Fogle, and A. Logan-Terry. 2008. "Family Language Policy." Language and Linguistics Compass 2 (5): 907-922.

King, K.A. and E. Lanza, eds. 2018. "Ideology, Agency and Imagination in Multilingual Families: An Introduction.” International Journal of Bilingual Education and Bilingualism 23 (3): 717-723.

King, K.A. and Flugel, S. in press. "Family Language Policy in Social Media: A Double-Bind for "good" Latino Parents."

La Vanguardia. May 23, 2018. "Hablar español es cada vez más peligroso en EE.UU." La Vanguardia. Accessed January 9, 2020. https://www.lavanguardia. com/internacional/20180523/443779277568/hablar-espanol-mas-peligroso-e euu.html

Lanza, E. (1997) 2004. Language Mixing in Infant Bilingualism. Oxford, UK: Oxford University Press.

Lanza, E. 2007. "Multilingualism in the Family." In Handbook of Multilingualism and Multilingual Communication, edited by P. Auer and Li Wei, 45-67. Berlin, Germany: Mouton de Gruyter.

Lanza, E. 2020a. "Urban Multilingualism and Family Language Policy." In Urban Multilingualism in Europe: Bridging the Gap between Language Policies and Language Practices, edited by G. Caliendo, R. Janssens, S. Slembrouck, and P. Van Avermaet, 121-139. Berlin: Mouton de Gruyter.

Lanza, E. 2020b. "Digital Storytelling: Multilingual Parents' Blogs and Vlogs as Narratives of Family Language Policy." In Språkreiser - festskrift til Anne Golden på 70-årsdagen, edited by L.A. Kulbrandstad and G.B. Steien, 177-192. Oslo: Novus Forlag.

Lanza, E. and X.L. Curdt-Christiansen, eds. 2018. "Special issue: Multilingual families: Aspirations and Challenges." International Journal of Multilingualism 15 (3): 231-232. doi: 10.1080/14790718.2018.1477091

Lanza, E. and Li Wei, eds. 2016. "Special Issue: Multilingual Encounters in Transcultural Families." Journal of Multilingual and Multicultural Development 37 (7): 653-654. 
Lanza, E. and K.V. Lexander. 2019. "Family Language Practices in Multilingual Transcultural Families." In Multidisciplinary Perspectives on Multilingualism: The Fundamentals, edited by S. Montanari and S. Quay, 229-252. Berlin, Boston: De Gruyter Mouton.

Lanza, E. and R. Lomeu Gomes. 2020. "Family Language Policy: Foundations, Theoretical Perspectives and Critical Approaches." In Handbook of Home Language Maintenance and Development. Social and Affective Factors, edited by A.C. Schalley and S.A. Eisenchlas, 153-173. Berlin, Boston: De Gruyter Mouton. Lomeu Gomes, R. 2020. “Talking Multilingual Families into Being: Language Practices and Ideologies of a Brazilian-Norwegian Family in Norway." Journal of Multilingual and Multicultural Development. doi:10.1080/01434632.2020.1 788037.

Lopez, M.H., A. Gonzalez-Barrera, J. M. Krogstad. October 25, 2018. "Views of Immigration Policy.” Pew Research Center. Accessed January 9, 2020. https:// www.pewresearch.org/hispanic/2018/10/25/views-of-immigration-policy/

Machado, A. (March 26, 2019). How the U.S Taught Me That Spanish was Shameful. NBC Universal. Accessed January 10, 2020. https://www.nbcnews. com/think/opinion/how-u-s-taught-me-spanish-was-shameful-ncna986746

My Latina Table. 2020. “Consejos para Criar Hijos Bilingües.” My Latina Table. Accessed January 10, 2020. https://www.mylatinatable.com/consejos-para-criarhijos-bilingues/

Purkarthofer, J., E. Lanza, and M. Finstad Berg. (forthcoming). “Transnational Families, the Media and the Norwegian Barnevernet: Discourses between the Public and the Private."

Robbins, L. May 16, 2018. “Man Threatens Spanish-speaking Workers: 'My Next Call Will be to ICE.'” The New York Times. Accessed January 10, 2020. https:// www.nytimes.com/2018/05/16/nyregion/man-threatens-spanish-language-video .html

Shearer, E. and J. Gottfried. September 7, 2017. "News Use Across Social Media Platforms 2017." Pew Research Center. Accessed January 9, 2020. https://www. journalism.org/2017/09/07/news-use-across-social-media-platforms-2017/

Sinclair, J. and M. Coulthard. 1975.Toward an Analysis of Discourse: The English Used by Teachers and Pupils. Oxford: Oxford University Press.

Song, K. 2019. “Immigrant Parents' Ideological Positioning on Bilingualism.” Theory into Practice 58 (3): 254-262.

Smith-Christmas, C. 2016. Family Language Policy: Maintaining an Endangered Language in the Home. Basingstoke: Palgrave Macmillan.

Smith-Christmas, C. 2018. “'One Cas, Two Cas:' Exploring the Affective Dimensions of Family Language Policy.” Multilingua: Journal of Cross-Cultural and Interlanguage Communication 37 (2): 211-230.

Southern Poverty Law Center. 2020. "Issues: Immigrant Justice.” Southern Poverty Law Center. Accessed January 9, 2020. https://www.splcenter.org/issues/im migrant-justice

Spolsky, B. 2012. "Family Language Policy - The Critical Domain." Journal of Multilingual and Multicultural Development 33 (1): 3-11.

Sugarman, J. and M. Lazarín. 2020. Educating English Learners during the COVID19 Pandemic: Policy Ideas for States and School Districts. Washington, DC: Migration Policy Institute. 
Surana, K. June 8, 2018. "How Racial Profiling goes Unchecked in Immigration Enforcement.” Pro Publica. Accessed January 19, 2020. https://www.propublica. org/article/racial-profiling-ice-immigration-enforcement-pennsylvania

Trump, D.J. June 17, 2019. “Next week ICE....” Accessed September 26, 2020. https://twitter.com/realdonaldtrump/status/1140791400658870274?lang=en

Univision. June 6, 2017. "Por qué debemos hablar español en público y con orgullo y no dejar que nadie nos silencie." Univision Noticias. Accessed January 16, 2020. https://www.univision.com/noticias/inmigracion/por-que-debemos-hablar-espan ol-en-publico-y-con-orgullo-y-no-dejar-que-nadie-nos-silencie.

Wofsy, C. and C. Borgmann. February 14, 2019. "U.S Border Patrol Detained U.S Citizens for Speaking Spanish in Montana.” ACLU. Accessed January 10, 2020. https://www.aclu.org/blog/immigrants-rights/ice-and-border-patrol-abuses/usborder-patrol-detained-us-citizens-speaking

Women's Refugee Commission. June 19, 2019. "Migrant Rights And Justice: Resources for Families Facing Deportation and Separation." Women's Refugee Commission. Accessed January 9, 2020. https://www.womensrefugeecommission. org/rights/resources/1409-resources-for-families-facing-deportation-separation 


\title{
5 Family Time(s)
}

\section{Migrant Temporalities in \\ Family Language Planning in the Urban African South}

\author{
Christine Anthonissen and \\ Christopher Stroud
}

\section{Introduction}

This chapter reflects on the ways in which questions in the research field of Family Language Policy (FLP), as an outcome of family language planning, are contextually determined. We refer specifically to international African migration in South Africa. Working critically with a definition of "family language policy" as the explicit and overt planning of language use within the home and among family members (King and Fogle 2017), we consider the choices families ${ }^{1}$ make regarding the language resources they have and which to prioritize in various social situations. The chapter discusses migrant-family language positions and practices against the background of an overt, de jure national language policy that recognizes equal rights for 11 South African family languages, ${ }^{2}$ and a covert, de facto policy that privileges English, as in the national education policy as well as in widespread public domain practices, e.g. in employment and in courts of law. Further, we bear in mind the increased linguistic diversity in the country due to migrancy from other African countries, particularly in the past ten years. We relate to Lanza and Li Wei $(2016,653)$, who turn attention to studies of diverse transnational family types with multilingualism as a dominant cultural feature, and to King $(2016,731)$, who refers to the need for FLP research to go beyond traditional family dispensations and consider the diversity of "globally dispersed, transnational populations." Gomes (2018) moves toward a critical approach in which research not only describes and explains FLP phenomena, but also critically responds and, on the basis of an enlarged data basis with an expanded scope "in terms of languages, countries and family configurations," impacts on FLP literature and could shift perspectives and practices. In this chapter, we wish to continue along this critical trajectory. We do so by considering a specific dynamic in the formation and maintenance of family structures, namely temporality, suggesting that time is not only a variable in family planning but also a determinant of family structure and the resources - material, institutional and otherwise - that are available to families. 
To understand the linguistic context into which migrant families enter on coming to South Africa in the twenty-first century, a history of human mobility of at least 400 years, European colonial and trade expansion since the mid-seventeenth century, and Apartheid rule of 40 years (which included mobility restrictions), needs to be considered. After the transition to democratic rule in 1994, an extensive language planning and policy project ended in a decision to extend the former two-languages policy that recognized only English and Afrikaans in official use. Thus, the early transition regulations already accepted the nine widely used (regional) indigenous languages additional to the two former colonial, Germanic languages. Since 1996, the official languages (listed in order of numbers of L1-speakers) are: isiZulu, isiXhosa, Afrikaans, Sepedi, English, Setswana, Sesotho, Xitsonga, siSwati, Tshivenda, and isiNdebele.

In what follows, we first very briefly consider what language planning and policy might mean in a contemporary world of enormous diversity and mobility, given that the basic framework for language planning on which FLP studies rest, emerged out of a much less complex context of diversity and human movement. Second, we elaborate on one construct that rarely gets attention, yet deserves more consideration in language planning and policy studies generally, namely that of "time" and temporality (Robertson and Ho 2016). The importance of temporality - especially subjective time for agency and choice is starkly highlighted by the types of families that we discuss here and that find themselves in often inherently chaotic, multiplex, intensely fluid, and precarious life situations. Third, we look closely at a small data-set of interviews and observations by way of analysis and illustration, in order to explore the kind of family language policies that would emerge in migrant communities where the notion of "family" requires new (ecological, including temporal) framings. Here, we also provide short biographical sketches of the families we discuss. Finally, by way of discussion, we introduce the notion of "vulnerability," suggesting this has implications for planning and policy. We follow the IFRC definition of "vulnerability" as a concept opposed to "capacity," which refers to "the diminished capacity of an individual or group to anticipate, cope with, resist and recover from the impact of a natural or man-made hazard." We anticipate that our chapter will add to the field by considering how the concept of FLP would be enacted and which critical features are pertinent in vulnerable migrant families whose recent histories are ones of south-south migration.

\section{Migrants in the African City}

Any planning endeavour presupposes an understanding of time as unfolding in a linear way; it also presupposes the mobilization of relevant institutional and other resources to pursue this unfolding. Planning anticipates submitting to forms of governmentality that work on emplacement and stable "presents" (Robertson and Ho 2016) with conditions in which investments 
(of shorter or longer duration) will reap the rewards in an anticipated (in the plan, definable) future, and in ways that are of significance and of relevance to those stakeholders who are doing the planning. It also assumes that the institutions and other structures (schools, workplaces, etc.) are accessible to stakeholders, and are understood to be in line with the aspirations and goals of the plan(ner), and/or are sufficiently flexible to meet the needs of potentially quite divergent actors. These conditions facilitate "explicit and overt" planning that will determine how language is used in the family setting.

For many African migrants in urban spaces such as Cape Town, many of these planning preconditions are far from being met - if any at all. The "families" that we discuss in this study, comprising of migrants from subSaharan African countries, such as Zimbabwe, the Democratic Republic of Congo (DRC), Malawi, and Mozambique, illustrate well some of the common ways in which the situation of the migrant in African urban spaces may differ from those commonly treated in extant FLP literature. Considering the African and South African migrancy context, our approach extends King and Fogle's (2017) definition to show that much planning and eventual fixing of an FLP is done implicitly, unreflectively, and therefore also covertly.

First, we need to recognize that crossing borders is not just a spatial journey, but, and perhaps more importantly for this study, a temporal journey. An important function of contemporary borders is to manage the flows and trickles of migrant labor in accordance with the movement and needs of transnational capital. One implication of this is that different migrant categories are subject to different (official) temporal regimes characteristic of (e.g.) short-term labour, exceptional skills residence permits, etc. (for more on such differentiation, see Li Wei and Kelly-Holmes 2021). We can see this as "time as governance" instantiated variously in institutions, workplaces, educational trajectories, etc., situationally referred to in this study.

The migrant experience is also oriented to time and temporality in other ways. Many migrants remain rooted in the past (the lives and families left behind), as well as being inclined toward the future. The past as past is thus very much part of the migrant present, e.g. in the form of remittances sent home, keeping in touch with children and other family members left behind, or waiting for accredited qualifications to arrive. Another axis of temporality defining migration is fundamental to the migrant experience itself, which is the idea of a "future" that is "better than the past." The migrant is often "assumed" to have goals and agendas for the future - better education, health, a good job, secure housing. For all sorts of reasons, fulfilling the hopes and dreams of a better future may be difficult - often the migrant has no job, no link to the formal economy, no relevant permits (many employers will not employ migrants who do not have a South African ID) making concrete, tangible goals of this type hardly attainable.

If we take the temporal experiences of the migrant as our point of departure - not least because of the centrality of time in the planning 
endeavour itself, we can discuss the migrant experience in terms of the salience of itineraries, provisionality, and improvised (uncertain) futures.

\subsection{Itineraries}

Many migrants live with "itineraries" (Best 2019; Simone 2019) characterized by short-term stays, short-term jobs, "a certain kind of circulation" - all "not easily governable within systems that rely upon notions of emplacement as a mode of governmentality" (Simone 2019). People occupy spaces of "provisionality" rather than permanence (Simone 2018; Best 2019). For most, the city where they first settle is not the place where they stay. For example, many coming to South Africa via Zimbabwe start in Johannesburg or Pretoria, but soon find their way to other urban and also semi-urban spaces. Especially for DRC migrants, Cape Town is such a stop en route to other places (cf. Vigouroux 2008, 2009), some with dreams of eventually finding their way to countries where their linguistic resources will have more currency, e.g. to France or Francophone Canada.

Personal histories of the migrants in our data-set all start out in areas of conflict, stress, and hardship, in conditions of civil war, political unrest, ethnic strife, poverty, or family disruption. The decision to move south is based on expectations of better life chances. All our respondents have travelling histories; none found exactly what they had imagined, yet most do not want to return. Some do not believe the situation back "home" will improve (e.g. civil unrest and social disruption in the DRC); others would like to return if political and economic conditions were to improve (e.g. to Zimbabwe, where people hope the economic devastation will be repaired).

Their trajectories from their home community to Cape Town tell stories of living in transient circumstances, along itinerant lines, paying intermediaries to make transport reservations, waiting for months in temporary arrangements dependant on the care or kindness of distant acquaintances or strangers, in anticipation of a bus ticket and some kind of certification for border crossings. Finally, some would mention they found their way to (e.g.) Maputo in Mozambique and then on to Durban. Seeking and not finding employment in Durban they take up contact with acquaintances, an uncle, a sister, in Cape Town and then move there. These are people for whom mobility and temporality are to a large extent a constant - they move between cities and towns, from one housing arrangement to another, between different kinds of employment - always on the move.

\subsection{Provisionality}

The itinerant nature of this type of migration leads to provisionality (Simone 2018). Always being on the move, the migrants pick up jobs where available in (e.g.) the security industry, the hospitality industry, as gardeners, housekeepers, in construction work; some try to make a living in entrepreneurial 
ways, setting up roadside kiosks as street vendors, hairdressers, musicians; others source help from close friends or relatives. Most wish for and even succeed in finding fragile stability, relying on networking and communicative skills, creatively making a living in a relatively hostile ecology. It is a "lucky" few who find options in the formal economy or institutional structures of the host country. These contingencies are led and reinforced by the fact that the largest group of recently relocated migrants come as young single men, less often also as single women, who travel over land by car or bus, in some cases also more and less covertly in large goods-transport lorries. The migrants we met in interviews all mentioned having come to metropolitan areas in the north and east of the country to join a family member or friend already in the country, at least as a starting point. These migrants form communities that could resemble family relationships, small groups with shared heritage in terms of where they started life, what their needs and aspirations are, the kinds of difficulties they run into, and finally also the language(s) they share. Starting out in South Africa, most rely on provisional accommodation, provisional forms of income, provisional rights to stay and make a living. Relationships to friends, family members, and accidental acquaintances of similar origin, give more security than any formal and institutional structures - also because they minimally engage with or receive support from such formal structures. They live out their daily lives in "polycentric, horizontal social relationships" (Landau and Freemantle 2016) in which they respond to "management from below (or beside), relying less on the coercive and normative power of state institutions, churches and regulated markets than on varying vernacular discourses" (Landau and Freemantle 2016).

\subsection{Improvised Futures}

The provisionality and itinerant lives of migrants also impact notions of the future. Migrants generally are future-oriented; however, once they have entered the South African marketplace, the hoped-for futures may be bleak or, realistically, even unattainable. In the absence of clearly defined futures, there is a focus on the concrete here and now, where agencies are informed by "radical hope" (Kallio, Meier, and Hakli 2020), a view of the future as "an unsettled horizon" (ibid), as a potentiality rather than a set agenda. Disillusioned expectations and battles to keep day-to-day life going tend to limit future perspectives and undermine decisive agency toward possibilities. Of course, the bleak future leads to an orientation to the "now" and the extra-systemic, institutional - the provisional grouping.

\subsection{Family Structure}

Thinking of the migrant in terms of different temporal regimes, we can distinguish different family structures as well as (and related to) different socioethnic-demographic-migrant structures, each marking different aspects of identity, all with relevance to language. Forms of temporality determine the 
migrant experience. Also relevant are how particular identities, ethnicities, and languages intersect with mobilities and temporalities to construct vastly differential experiences of what aspirations are possible, which material conditions will structure family life. There are of course immigrant families that fit a traditional pattern according to which a family relocates from (e.g.) Zimbabwe or Malawi on the basis of one or both parents having gained lucrative employment (e.g.) as an accountant, university lecturer, or health care professional. However, more often, the temporariness leads to disrupted and vulnerable family structures. Many leave the closest family, such as their wife and parents behind; others leave children in the care of grandparents or other close relatives, thus disrupting traditional nuclear as well as extended family structures.

The provisionality among migrants of not staying too long in any particular place also has an effect on the kind of family life migrants can imagine. Without a stable income, a fixed address - how does one start a family, how does one plan for the future of children, what kind of family intimacy is possible? Many who leave children in their country of origin intend to send for them, bring them to join the parents once established in South Africa. However, for a sizeable majority, such stability is hardly attainable. Particularly, as mentioned above, adult migrants whose English-language skills prohibit the social mobility and relational stability they came after in leaving "home," imagine finding their feet by moving on to more stability in yet another country.

The provisional and temporary nature of this common migrant experience also finds reflection in family structure with respect to children who come with their parents or are born after their parents arrived and "settled" in South Africa. Many come to South Africa on their own, find long- or short-term partners, and have children born into precarious family circumstances. As we will see in Section 4, such temporarily defined family structures contribute powerfully to the profiles of FLP.

\subsection{Migrant Children's Access to Education}

The itinerant, provisional, and improvised futures of migrant families also resonate in the educational and other structural child care provisions on offer and accessible to these families (on similar exclusion practices, see Li Wei and Kelly-Holmes 2021). Although there is a well-developed legal and policy framework for the protection of migrant children's rights in South Africa, regardless of their documentation, ${ }^{4}$ a relatively large body of work on the rights of migrant children demonstrates the limited protection they often get globally, as well as in South Africa (see, e.g. Stephens 1995; Babha 2009; Palmary 2009; Van Baalen 2012; Washinyira 2019). Reports on the marginalization and discrimination of migrant children are not uncommon. Considering migrants of African descent in southsouth migration into South Africa, Vigouroux's $(2008,2009)$ interviews 
with 154 migrants from Francophone African countries into Cape Town touches on family life and children's education as only one of the various kinds of settlement difficulties. Similarly, Landau and Pampalone's (2018) interviews with migrants in the Gauteng region give very little on children and provision for their education. On the one hand, Chae, Hayford, and Agadjanian's (2016) longitudinal study in rural Mozambique confirms that paternal labour migration, in which children are left in the home environment, is widespread and increasing; on the other hand, the numbers of children living precariously due to the precarity of their parents' circumstances are obscured. Rarely mentioned are the perilous circumstances of unaccompanied migrant children (see, e.g. Skelton and Ngidi 2014; Zhou 2018), often young boys, who find their way into the informal economies around major urban centres.

In spite of relatively progressive protective legislation, also in the National Education Policy Act (27/1996), admission to primary and secondary schools is often discriminatory. As recent as in March 2019, Killander (2019) gave evidence of how migrant children's access even to basic education is limited, either due to their parents or guardians not having secured their child's required identity documents, or due to their inability to pay school fees, even in no-fee designated state schools (cf. also Van Baalen 2012; Washinyira 2019).

\section{Family Language Planning and Multilingualism in Migrant Families}

We have noted in the preceding section a number of dimensions revolving around temporality that distinguishes migrant families in the South African context from "prototypical" families - if such exist (although we also recognize diversity in the prototype). Coetzee (2018) refers to such a-typical families in sub-economic settlements in Cape Town, showing not only the diversity of language practices in such indigenous multilingual communities but also indicating how disrupted family life is not a feature only of precariously situated migrant families. What, then, do we find with respect to the FLP of migrant families of whom we met representatives in the Cape Town metropolitan area in South Africa? We introduce a number of case studies, in which various matters relevant to their FLPs become evident. The cases selected here show (i) the effects of fluid temporality, of families constantly being on the move, of unstable housing conditions, (ii) the precarity of vulnerable migrant families vs. the security of those more socially secure, and (iii) the role schooling plays as a means of improving family life chances, highlighting again the accidental way in which FLP develops in migrant communities with exceptionally low socio-economic status due to their transience. The case studies highlight the effects these social conditions have on family structures and related FLPs, highlighting how implicitly and coincidentally FLPs are developed. 
We refer to material collected in two focus group discussions and three personal interviews on various occasions between November 2015 and December 2018, in which migrant parents and caregivers from such migrant communities in and around Cape Town referred to their own language repertoires, those of their children, and thus also disclosed multilingual family language policies. The data presented here was collected by one of the authors, with assistance from four postgraduate students in the course of projects where our main interest was not in FLP, but in the value and use of the linguistic repertoires African migrants bring to the region. We used the same meta-data form for all participants, thus ensuring that similar kinds of data were elicited, even if at different times. The discourses on the trajectories that brought the participants to South Africa only partially referred to children and their part in the migrancy experience. Nevertheless, either in passing (Focus group 1, with hospitality industry workers), or in response to direct questions (Interviews 1 and 2, with participants at a training centre, selected on the basis of their contribution to a foregoing, second focus group discussion), references to children's education gave clues to the kinds of FLP that were either in place or in planning.

\subsection{Case Studies}

Case 1: Sewing and Hairdressing - Parents at a Community

Training Centre Jacob, ${ }^{5}$ a hairdresser from the DRC, has been living in Cape Town since 2003. Besides the six Congolese languages he knows (Bembe, Kikongo, Kiswahili, French, Lari, and Lingala), in migrating to South Africa he added Portuguese, English, isiXhosa, and even a few words of Afrikaans. Although he had, at the time of the interview, been in the country for 13 years, he still had refugee status with the limitations such a status holds in relation to citizenship rights. He provides for himself and his family (i.e. his wife, also from the DRC, and two young children, both born in South Africa) by facilitating skills development in hairdressing and sewing at a small training centre for "whoever comes," i.e. foreigners as well as local unemployed trainees. Jacob introduced $u^{6}$ to a number of other migrants (snowball sampling) of whom some work at the centre. One of these, Claudette, has two South African-born children (aged 7 and 2 years old). The older daughter (7 years old) attends a local state school where the language of learning is English. Their home language is French. Thus the de facto FLP maintains the mother's DRC French at home, while the children's development of the language of education becomes the schools' responsibility. Claudette has low English proficiency; in communication with us she was reticent, would give answers and comment in a few English words, and then switch to French, looking at one of the other participants to relay her contribution. Both her children spend 8-9 hours per day at school, after-school, and in daycare, as Claudette works long hours. She does not reflect on the 


\section{2}

education of her children in terms of rights; thus, in response to our questions about enrolment or language support, she was not demanding. She is proud of the way in which her first-born has learnt English, and is probably already more proficient in this local lingua franca than she is herself:

No, she's good. She can read ... write.

Asked whether the child sometimes interprets for her, she laughed and answered:

Yes, she can. Sometimes.

A second respondent at Jacob's training centre, Myriam, gave an account of considerably more dire circumstances. She had come to South Africa from Malawi a couple of years earlier, leaving the house of a harsh uncle who had been appointed as her guardian after the death of both her parents. She has a South African-born 7-year-old son who is currently registered in an Afrikaans-medium school in a historically "coloured," lower-middle-class area. Myriam has an extremely irregular income, and moves house often as she can rarely keep up with the rent, which means she has to find new accommodation when she gets evicted. Her son is battling at school to the extent that she had been summoned to meet the principal more than once in the foregoing months, mostly to address her son's irregular attendance, which Myriam says is due to difficulties finding suitable and affordable transport. She has very little to give in terms of participating in her child's formal education (see also Matchet (2002) on problems of literacy in disadvantaged communities in South Africa). The de facto FLP that Myriam follows is one of using a mixture of Chichewa and English with her son at home. Although the school does have an English-medium class, the majority of the children, as well as the class teacher, are Afrikaans L1 speakers. When asked whether the boy's English is developing in line with the school's expectations, and whether he is informally building Afrikaans language skills, Myriam's answer was vague. She offered an excuse we interpreted as one explaining his poor progress - probably not only in developing Afrikaans:

He is ok. Ehh ... I can't get taxi, then he can't go to school.

A third respondent, Sophie, represents a family from Burundi, where although the majority language is Kirundi, their family language was Swahili, while the language of learning and of prestige more widely, is French. Although Sophie indicated that she came to South Africa wanting to further her tertiary studies, she also mentioned that she lives with her 
brother, who runs an informal business, had invited her to South Africa to help with the care of his two small children (a boy aged one and a girl aged 5 years old), after his estranged wife had left, returning to Durban where the family had lived before moving to Cape Town. Regarding the languages used in this family, Sophie mentions that the adults speak Swahili, but that they speak English to the children following the language of the creche and the school. Therefore here, the FLP determines a heritage language between the adults, but the local lingua franca between adults and children. Asked whether the children had learnt English from their parents or from her, she said:

No! Not from me. Eh ... I don't know where ... from neighbours.

On the children's ability to follow the adult's Swahili, at first she said they don't understand, but later:

The little one, she's trying, but / not good ...

In an interview with Jacob and his wife, regarding the home language practices, Jacob mentioned first that his wife was not progressing well with learning English. Regarding the children's repertoires, he seemed pleased that their multilingual repertoires were developing well:

I'm teaching them with my mother tongue, Kibembe ... Swahili. // Those are the two languages I want them to speak ... If I say something in Kibembe, they hear; if I force them to talk, they can come after me. Their mother, she can speak Kibembe, but most of the time she speaks with them - Swahili.

Now at school, creche ... I'm taking them to one of the Zimbabwean creches.

Most of the children they come from / Zimbabwe. There, they are speaking only English. So at school - the little ones - all English.

Case 2: The Hospitality Service Industry - Parents Employed as Waiters Goodwin was interviewed ${ }^{7}$ after coming from duty at his workplace, a well-established restaurant and winery. He moved to South Africa from Zimbabwe shortly after the economic meltdown of the early 2000s. Trained as an electrician, he could find work neither in his home country nor in South Africa. A family member, on the basis of him having a driver's licence, introduced him to the support staff of a big hotel, where he gained the kind of experience that assisted in getting the current appointment. After a number of years his wife and two children joined so that they now live in relative comfort. 
The grandparents looked after them when they were still home. But it's better for children with their parents.

The children attend a local state school where the language of learning used to be Afrikaans only, which has recently added a number of English-medium classes for children whose parents are L1 speakers of languages other than English, but elect to have their children educated in English. Goodwin is satisfied with the opportunities his children get: there were no enrolment difficulties, and he finds the schooling and after-care good. Regarding FLP, their home language is Shona, thus not one that assists in integrating with the isiXhosa-dominant community in which they live. However, there is a supportive group of ex-Zimbabweans who alternate between Shona and English in their community interactions. Therefore, Goodwin and his family conduct family life through Shona L1, relatively good L2-English, and some receptive isiXhosa proficiency. He is entertained and impressed by the fact that his eldest has a number of Afrikaans L1 friends with whom he speaks a local variety of Afrikaans quite fluently.

He visits friends in Ida's Valley; they speak only Afrikaans. (laugh)

In Goodwin's case, the family repertoire of Shona and English has been extended through their migration experience. Specifically, the children have added conversational proficiency in Afrikaans, and receptive proficiency in isiXhosa.

Case 3: Financial management - mobile, well-qualified parents

An interview of a completely different kind took place with Gloria, ${ }^{8}$ a mother of three boys, originally from Zimbabwe whose trajectory with her husband, an accountant, has taken the family to various places in South Africa and on a two-year stint to Egypt and back. The family moved to South Africa when the eldest was 2 years old and at the time could speak the family's Shona L1 fluently. However, when he started attending pre-school in South Africa, he understood no English, and to assist him with fitting in the parents started to speak English at home. Now, ten years later, according to the mother, all three children can speak and understand English only, even while the parents speak Shona to each other. This family's socio-economic security translates into expressed interest in their children's education and how language can facilitate learning, social interaction, as well as attachment to a Shona heritage. Gloria believes that her teenage children's English is "way, way better than mine ... ten times better," and she is proud that her 9-year-old's teacher reported to her that his English and spelling "is of a 12-year-old." She has requested that her first-born be exempted from the compulsory Afrikaans lessons in secondary school - not because she objects to Afrikaans generally, but "because I cannot help them in any way." 


\subsection{Participants' Critical Engagement: Who is Supposed to Help the Children?}

In Jacob's narrative he referred to his child who was born in the local Karl Bremer Hospital and now is 7 years old, who, on the basis of her father's status, has a birth certificate that identifies her as a refugee. Jacob took initiative in resisting our narrow research agenda, changing the topic from interesting linguistic repertoires to what he found to be the burning questions. Regarding their children's education he asked:

What about the children? Who helps them with their homework? We [the parents] are supposed to help them. But we are broken ... [referring to his non-standard English]

In fact, Jacob was quite impatient about how little our academic interest in foreigners' linguistic repertoires offered; his message, summarized, was:

You are citizens; you have a position from which to speak; you should address these people who do not listen to us, who do not want to accept us here. You can call us "foreigners" and "refugees" - but what about the children?

By the end of the first focus group discussion, Jacob changed the multilingual biography discourse into a discourse of resistance. Although he was the most fluent and animated speaker in the group, other participants joined in, quite vocally reminding the researchers and one another of their social realities, and how various discourses are connected, one embedded in the other.

The generous national language policy in South Africa that affords 11 native languages official status, provides little official support in transferring from non-indigenous home languages to the local language of education. This responsibility is implicitly transferred to parents, or unofficially, to empathetic, concerned teachers. Besides language and literacy development (Mafandala 2014), other concerns regarding integrating migrant children and their parents into an enabling schooling system, are rarely addressed. At best, families and their children are invited to join in; at worst, they are refused access on the grounds of not having documentation that justifies their use of the educational resources. These social circumstances contribute to developing FLPs that in some cases maintain and perpetuate the heritage languages (as in the cases of Jacob and Goodwin), in other cases give up the heritage language in favour of developing English (as in the case of Gloria), and in some bring extended repertoires for the children, even if not for the parents (as in the cases of Claudette and Goodwin) (see also Li Wei and Kelly-Holmes 2021). The socially more vulnerable families can hardly attend to language development, even of the parents' L1, as in the cases of Sophie and Myriam. These cases confirm that planning family language(s) 
is a luxury in the migrancy condition - vulnerable respondents preferred telling their stories of hardship to merely reflecting on which languages they use when.

\subsection{Summary: Different Heritage Communities, Different Experiences, Different FLP Outcomes}

The following sections will illustrate the various dimensions we recognized, referring to the discursive contributions of specific migrant parents regarding their language identity, linguistic repertoire, and how these tie in to possible FLPs they would adhere to, even if rarely explicitly contemplated. We augment the excerpts above with complementary information from the focus groups. All recorded data is in English, an L2 for researchers as well as participants. However, participants' L2 skills were of varying competence levels: notably, migrants from Zimbabwe, Malawi, Nigeria, and Tanzania (former British colonies) were more able to clearly articulate their experiences than those from the DRC where French is the lingua franca and language in education. Nevertheless, relying on ten Thije's (2018) concept of "receptive multilingualism" in reference to such conversations in which speakers use different levels of the lingua franca and draw on the range of their linguistic resources, we achieved considerable communicative success (measured in terms of mutual agreement) in the given settings.

First, as we noted earlier, particular identities (ethnic, social class) and related background factors (education, languages) work in tandem with temporalities of migration. We note that where the migrants come from shapes language identities and FLPs. Migrants' national identities, their heritage communities, contribute in more and less obvious ways to their ability to establish as families in the Western Cape. This was evident in shared experiences, as well as differences between respondents from the DRC, Nigeria, Zimbabwe, Malawi, and Mozambique.

The distinction is marked between migrants from anglophone countries where the education system cultivates English-language and literacy skills (Zimbabwe, Malawi, Tanzania, Nigeria) on the one hand, and those from Francophone (DRC, Burundi, Rwanda) or Lusophone countries (Mozambique, Angola) on the other hand, where populations are multilingual, but with low English competence. Respondents of DRC origin, for example, experienced their rich repertoires as very limiting, many indicating that they did not anticipate the challenge of settling in an Englishdominant context. At first, meeting with researchers interested in language was awkward, as participants could not envisage the relevance of language considering the other pertinent things they (i) had to manage and take care of, and (ii) would prefer to raise with South Africans perceived to be in secure and powerful social positions. Thus, at first, we found little resonance when discussion was on repertoire, language biography, or language planning. 
Regarding language repertoire and language planning, most respondents were unprepared for such a question. They appeared not to think of themselves in terms of multilinguals with "linguistic capital." Respondents from Zimbabwe (e.g.), when prompted, would acknowledge the advantage of their fluency in L2-English, although they associated their employability more with their willingness to commit to job requirements, and at times also to accept relatively low wages. In contrast, respondents from the DRC would confirm the difficulties of language and accent marking them as foreigners, and of being found unsuitable for professions that require basic or even advanced communicative skills. Some expressed aspirations to improve their English, though as adult learners who needed to find a means of income, formal language learning was never an option. In temporality terms - only a very few could afford to spend six months in courses or in libraries trying to advance their English-language skills. Ranking their difficulties, however, worries about documentation, personal safety, and opportunities for upward mobility, seemed to be of greater concern than language. All of this, of course, is the result of living in provisionality.

Interestingly, though, possessing a broad repertoire and having language skills appeared to be one factor that could literally "buy time" for some migrants in the sense of providing more opportunities. In assessing their own competence, the relative terms in which many consider English proficiency became clear. Jacob, as a French-educated DRC citizen, could speak English considerably more fluently than Claudette, a highly skilled designer and tailor in the training shop, also from the DRC. Jacob's English gave a degree of job security and flexibility, while Claudette experienced very limited employment opportunities. Her work in the tailoring business - cutting, sewing, training - with Jacob as her supervisor, was highly valued though she would have to remain in the setting where communication through DRC French was possible. Possessing language skills is one way in which the scope of the local agency was expanded in opening up some future of "radical hope" mentioned above.

Second, regarding parents' engagement in their children's language learning, those with more permanent and less transient migrant conditions through, for example, income security were in a good position to reflect on FLP, to consider which languages to maintain, which ideally to develop for their children, which to select in deciding on schooling, which even to "sacrifice" in the process of preparing for suitable future employment. In contrast, the ills of itinerant and provisional lives, such as having to battle with employment uncertainty, low wages, insecure housing arrangements, official papers, and staying safe in the face of sporadic xenophobic violence, pushes language issues to the periphery - perhaps not surprisingly.

Considering FLP in migrant families, there are at least two kinds of structures, namely children left behind in the parent's home country to be cared for by family or extended family members, and those in the care of their foreigner parents in South Africa (cf. Palmary 2009, 3). For example, 


\section{8}

\section{Christine Anthonissen and Christopher Stroud}

large numbers of migrants of Zimbabwean origin with matric-level education leave their families at a young age, anticipating employment with an income large enough to sustain them in South Africa while also taking care of parents and siblings, a spouse, and children left behind in their home country. In contrast, migrants of Congolese origin who were schooled in French, face considerably greater difficulties in finding employment and a sustainable income. Many underestimate the difficulty of finding lucrative employment without English fluency. Some studies report on the advantages for left-behind children if the migrating parents are able to send home remittances (cf. UNICEF paper 2009/10); in substitute care, migrant FLPs are not directly relevant, although the absence of the parent is not without effect.

Third, regarding planning family language vs. school language(s) - to take part in planning one has to be included. FLP literature in a global northern context does reflect diversity in the integrating and accommodating practices followed in different contexts; yet it generally presupposes that institutions in multilingual communities will recognize (to some extent) the family language, and in considered contexts also plan for bridging between home and school languages, as with Spanish in the USA, Welsh and English in Wales, Finnish in Sweden, etc. Claudette's daughter's school has neither teachers nor programmes prepared for learners with non-SA languages as L1s. The teacher appointed to support the English literacy of learners with migrant-family backgrounds (e.g. from DRC, Somalia) mentioned that parents are not confident in interacting with the school, so that although they register their children there they cannot support them at home. These parents, reportedly, very rarely attend parent-teacher meetings, possibly intimidated due to their own limited English or assuming they have no role in assuring their child's progress. ${ }^{9}$ Further, some schools are reluctant to enrol students with migrancy backgrounds (as indicated above), so that once a child is enrolled, parents could be hesitant to jeopardize the child's access by exhibiting their "foreignness." Decisions about which family language will be introduced in such families, which kind of schooling would be ideal, and which SA languages the children would be introduced to, are often taken only by default.

\section{Discussion}

The unsettled families in our study (i) do not fit traditional family structures, (ii) are highly mobile with limited perspective on stability in terms of location, family income, settling children in formal education, and (iii) hardly reflect on "language matters" in a context where apparently more existential issues such as accommodation, daily livelihood, dealing with police and internal administration, or xenophobic violence, confront them. As noted, these are families that live with the affordances provided by short-term itineraries, characterized by provisionality and uncertain, non-determinable 
futures. They are highly vulnerable families in many respects. We have wanted to suggest that bilingualism and multilingualism as enacted in such migrant communities differ largely from those in more secure contexts, where there is structural - even if often unsociable - support for migrants with traumatic backgrounds.

From the data, we can conclude that these socially vulnerable migrant families have language policy directives that are quite different to those of socially secure families who have time and material resources to invest in "family language planning." Ideologies on official languages, on (e.g.) which languages have value in finding lucrative employment and therefore should be promoted for and in education (cf., e.g. King and Lanza 2017), often reinforce the value of resources accessible to "upwardly mobile" families. Linguistic resources of the vulnerable (even if mobile) communities (Spencer, Clegg, and Stackhouse 2012) are mentioned in national policies that promote multilingualism, but they often stop at lip-service (cf. Van Baalen 2012; Mafandala 2014). Even so, for many migrants consideration of various options seems to be irrelevant - a luxury quite removed from their everyday lives.

Existing research (cf. Spolsky 2004, Shohamy 2006) indicates how vulnerable families, which include migrant families, are rarely supported with good information on home language vs. the languages used in education, developing bilingual skills, learning the official (dominant) language, and simultaneously maintaining family languages (Nieto 1987, Fishman 1970, Genesee 2013). Narrative data in the various publications, and from semistructured interviews (De Fina 2009) also informs what happens in planning and executing family language policies among migrants from central and southern Africa, in urban contexts in South Africa. The presupposition that vulnerable migrant respondents will view language as a resource and would see language as an explicit part of their identity if not directly questioned has to be interrogated.

Although characterizing "family" - including non-traditional families - in FLP research is not a new suggestion (see, e.g. King 2016, King and Lanza 2017, Gomes 2018), focusing on "vulnerability" does add an important dimension. We have suggested that a core feature of these migrant families that needs more consideration is how their vulnerability can be correlated with the temporal regimes under which they live and with which they struggle. Different migration profiles in terms of aspiration, orientation, and structure produce a diversity of 'family' profiles, more or less vulnerable. The most vulnerable migrant communities not only extend our understanding of "family," they in fact identify family life and relationships as a site of disruption and even trauma. Imagining family and imagining personal futures are contested grounds, areas of uncertainty and insecurity. The temporal regimes determine what choices might even be relevant. At the very least, migrant families hardly have 
the time or capacity to reflect on the available choices (cf. Yabiku and Agadjanian 2017).

There are also methodological implications that follow from a more explicit reflection of the temporal life conditions of migrants. For example, it would seem desirable to revisit the types of question we ask, and to question what is appropriate and productive, and what is not. How do we take structures of anticipation and mobility of the respondents into account when engaging in linguistic modes of enquiry? The vocabularizing of linguistic identity can be an alien and alienating discourse. These are just a few implications that follow from family temporalities of precarity.

\section{Conclusion}

In this chapter, we have focused on a particular instance of FLP, that of African migrants' within Africa, and one significant dimension of FLP, namely temporality. Temporality is the subjective and lived experience in time, and the manner in which the "African migrant" orientates to time. Temporality is important for a number of reasons we have pointed to above. Two ways that stand out as particularly relevant for family language planning are first provisionality - lack of engagement with formal structures - and an uncertain future. And second, planning itself is a temporal practice - albeit one clearly tailored to the temporal regime of linear (national) time - from which many in this group are excluded.

We have illustrated the nature of the journeys of the migrants in terms of their structured identities, expectations, and possibilities, although these are diverse across individuals and groups. We have considered the profiles of highly mobile and often greatly disrupted migrants as they are given in the literature, media reports, as well as a number of personal interviews, and sought answers to how the concept of "family language" features, which choices (explicit or implicitly, conscious or unconsciously) such families make in terms of family language vs. community languages and language-in-education. We have argued that raising the theme of language, linguistic diversity, language repertoires, different competences, and finally also family language choices, preferences, and ideologies in such communities, promises a range of new perspectives. Specifically, reflecting on these migrant communities in terms of family and FLP raises questions with the potential to extend the research field of FLP, particularly at the intersection with sociolinguistics, social anthropology, and critical linguistics.

Perhaps the long and the short of it is that you cannot plan for the future unless you have one, and you cannot plan for equitable futures when you do not have the means - or access to the means (infrastructures, institutions) to make them happen. We acknowledge that this is hardly a novel insight, but one that nevertheless seems not to resonate loudly in policy and planning documents or even in much research literature. 


\section{Notes}

1 Regarding "family" we take this term as one that designates a diversity of social forms, of parents and children living together as a unit (Beck-Gernsheim 2002), including also groups constituted by one adult member and one or more others, where there is a generational connection, and members are related by birth, marriage, or adoption and residing together, working towards mutual need fulfilment, nurturance, and development (Edwards and Graham 2009, 193).

2 For a more elaborate description of linguistic diversity and the inclusion of all South African home languages in the official language policy, see Anthonissen (2010, 109-113).

3 The International federation of Red Cross and Red Crescent Societies, a humanitarian network with primary aim to reduce vulnerability, also by reducing discrimination, increasing resilience and fostering a culture of peace globally. See https://www.ifrc.org/en/who-we-are/

4 Also, South Africa has accepted a number of UN directives intended to secure the social and educational rights of refugee children (Van Baalen 2012).

5 All respondents' names have been changed to protect their identity.

6 Special thanks to Lauren Mongie, Carla Roets, Philip Steyn who assisted with recording the focus group discussions.

7 Thank you to Erica George who assisted with recording the interview.

8 Interview jointly conducted by CA (author) and Elizabeth Lanza.

9 See Grolnick et al. (1997) and Flouri (2006) regarding a correlation between poor social support to parents and less personal involvement in the child's schooling.

\section{References}

Anthonissen, C. 2010. "Managing Linguistic Diversity in a South African HIV/ AIDS Day Clinic.” In Multilingualism at Work, edited by B. Meyer and B. Apfelbaum. Amsterdam: John Benjamins.

Beck-Gernsheim, E. 2002. Reinventing the Family: In Search of New Lifestyles. Malden, Mass.: Polity Press.

Best, A. 2019. “Interview with AbdouMalik Simone.” Accessed May 20, 2020. https//:societyandspace.org/articles/on-improvisation-southern-urbanism-andrhythms-of-the-everyday

Babha, J. 2009. “Ahrendt's Children: Do Today's Migrant Children Have a Right to Have Rights?” Human Rights Quarterly 31: 410-451.

Chae, S., S.R. Hayford, and V. Agadjanian. 2016. "Father's Migration and Leaving the Parental Home in Rural Mozambique." Journal of Marriage and Family 78: 1047-1062.

Coetzee, F. 2018. "Hy leer dit nie hier nie ('He doesn't learn it here'): Talking About Children's Swearing in Extended Families in Multilingual South Africa.” International Journal of Multilingualism 15 (2): 1-15.

De Fina, A. 2009. "Narratives in Interview - The Case of Accounts." Narrative Inquiry 19 (2): 233-258.

Edwards, A.P. and E.E. Graham 2009. "The Relationship between Individuals' Definitions of Family and Implicit Personal Theories of Communication." Journal of Family Communication 9 (4): 191-208.

Fishman, J. 1970. "Bilingual Education in Sociolinguistic Perspective." Unpublished paper as TESOL Convention, California. https://files.eric.ed.gov/fulltext/ED040 404.pdf 


\section{Christine Anthonissen and Christopher Stroud}

Flouri, E. 2006. “Parental Interest in Children's Education, Children's Self-esteem and Locus of Control, and Later Educational Attainment: Twenty-six-year Follow-up of the 1970 British Birth Cohort." British Journal of Educational Psychology 76: 41-55.

Genesee, F. 2013. "Insights into Bilingual Education from Research on Immersion Programs in Canada." In Bilingual and Multilingual Education in the 21st Century, edited by C. Abello-Contesse, P.M. Chandler, M.D. Lopez-Jiminez, and R. Chacon-Beltran. Ontario: Multilingual Matters.

Gomes, R.L. 2018. "Family Language Policy Ten Years on: A Critical Approach to Family Multilingualism.” Multilingual Margins 5 (2): 51-72.

Grolnick, W.S., C. Benjet, C.O. Kurowski, and N.H. Apostoleris. 1997. "Predictors of Parent Involvement in Children's Schooling." Journal of Educational Psychology 89 (3): 538-548.

IFRC organizational webpage. 2020. “What is Vulnerability?” International Federation of Red Cross and Red Crescent Societies. https://www.ifrc.org/en/ what-we-do/disaster-management/about-disasters/what-is-a-disaster/what-is-vu Inerability/ Accessed August 5, 2021.

Kallio, K.S., I. Meier, and J. Hakli. 2020. "Radical Hope in Asylum Seeking: Political Agency Beyond Linear Temporality." Journal of Ethnic and Migration Studies. doi:10.1080/1369X.2020.1764344.

Killander, M. 2019. "SA is Failing the Rights of Children's Access to Education and Health." Rand Daily Mail, March 6, 2019. Accessed March 4, 2021. https://ww w.businesslive.co.za/rdm/news/2019-03-06-sa-is-failing-the-rights-of-childrens-a ccess-to-education-and-health/

King, K. 2016. "Language Policy, Multilingual Encounters, and Transnational Families." Journal of Multilingual and Multicultural Development 37 (7): 726733. doi:10.1080/01434632.2015.1127927

King, K. and L.W. Fogle. 2017. "Family Language Policy.” In Language Policy and Political Issues in Education. Encyclopedia of Language and Education, 3rd edition, edited by T. McCarty and S. May. Cham: Springer.

King, K., and E. Lanza. 2017. "Ideology, Agency, and Imagination in Multilingual Families: An Introduction." International Journal of Bilingualism 23 (3): 717-723.

Lanza, E. and Li Wei. 2016. "Multilingual Encounters in Transcultural Families." Journal of Multilingual and Multicultural Development 37 (7): 653-654. doi:10. 1080/01434632.2016.1151198

Landau, L.B. and I. Freemantle. 2016. "Beggaring Belonging in Africa's No-man's Lands: Diversity, Usufruct and the Ethics of Accommodation." Journal of Ethnic and Migration Studies 42 (6): 933-951. doi:10.1080/1369183X.2015.1126086

Landau, L.B. and T. Pampalone. 2018. I Want to go Home Forever. Johannesburg: Wits University Press.

Li Wei and H. Kelly-Holmes. 2021. "Language Policy: Taking Stock in a Changing Context." Spaces of Multilingualism, edited by R. Blackwood and U. Røyneland. London; New York: Routledge.

Mafandala, J.M. 2014. “Congolese Migrant Learners' Language, Literacy and Learning in Primary School in Cape Town." Unpublished MA dissertation, University of Cape Town.

Nieto, S. 1987. "Parent Involvement in Bilingual Education: Whose Responsibility Is It?" National Association for Bilingual Education (NABE) Journal 11 (3): 189-201. 
Palmary, I. 2009. “For Better Implementation of Migrant Children's Rights in South Africa." UNICEF occasional paper. Accessed April 2, 2020. https://www.unicef. org/southafrica/SAF_resources_migrantchild1.pdf

Robertson, S. and E.L. Ho. 2016. "Temporalities, Materialities and Connecting Locales: Migration and Mobility in Asia-Pacific cities." Journal of Ethnic and Migration Studies 42 (14): 2263-2271. doi:10.1080/1369183X.2016.1205804

Shohamy, E. 2006. Language Policy: Hidden Agendas and New Approaches. London; New York: Routledge.

Simone, A. 2018. "Inoperable Relations and Urban Change in the Global South." In The Global South and Literature, edited by R. West-Pavlo, 123-133. Cambridge UK: Cambridge University Press. doi:10.1017/9781108231930.010

Simone, A. 2019. Improvised Lives. Rhythms of Endurance in an Urban South. Cambridge: Polity Press.

Skelton, A. and K. Ngidi. 2014. "Unaccompanied Foreign Migrant Children in South Africa." Policy Action Network (PAN), HSRC and UNICEF Topical Guide. Accessed March 29, 2020. https://children.pan.org.za

Spencer, S., J. Clegg, and J. Stackhouse. 2012. "Language and Disadvantage: A Comparison of the Language Abilities of Adolescents from Two Different Socioeconomic Areas." International Journal of Language Communication Disorders 47 (3): 274-284.

Spolsky, B. 2004. Language Policy. Cambridge, UK: University of Cambridge Press.

Stephens, S., ed. 1995. Children and the Politics of Culture. Princeton: Princeton University Press.

ten Thije, J. 2018. "Receptive Multilingualism." In Twelve Chapters on Multilingualism, edited by D. Singleton and L. Aronin, 329-263. Bristol: Multilingual Matters.

Van Baalen, C. 2012. “The Rights of Refugee Children in South Africa.” Unpublished MA (Law), North-West University, SA.

Vigouroux, C.B. 2008. "The Smuggling of la Francophonie. Francophone Africans in Anglophone Cape Town (South Africa).” Language in Society 37 (3): 415-434.

Vigouroux, C.B. 2009. “A Relational Understanding of Language Practice: Interacting Times-Spaces in a Single Ethnographic Site." In Globalization and Language in Contact: Scale Migration Flows, and Communicative Practices, edited by J. Collins, S. Slembrouck, and M. Baynham, 62-84. London: Continuum Press.

Washinyira, T. 2019. "Refugee Children Turned Away by Cape Schools." GroundUp. Accessed March 22, 2019. https://www.groundup.org.za/article/refu gee-children-turned-away-cape-schools/

Yabiku, S.T. and V. Agadjanian. 2017. "Father's Labour Migration and Children's School Discontinuation in Rural Mozambique." International Migration 55 (4): 188-202.

Zhou, B.S. 2018. "The Challenges of Undocumented Refugee Children in Accessing the Right to Basic Education in South Africa." Unpublished MA thesis, NorthWest University, South Africa. 


\title{
6 From "Civilising Missions" to Indigenous Language Reclamation
}

\author{
Language Policy, Language Shift, and \\ Maintenance in Australia and Norway
}

\section{Pia Lane and Gillian Wigglesworth}

\section{Introduction}

Indigenous peoples around the world share a history of marginalisation, oppression, silencing, and even displacement. Stringent assimilation policies in combination with processes of modernisation and economic development have led to language shift, as adults stop using their mother tongue with the next generation. Many nation-states set out on "civilising missions," portraying Indigenous populations as inferior and their languages as primitive. Such representations often became tools of government serving the colonisers' interests in extending military, economic, political, and cultural control (Lane and Makihara 2017). Educational systems came to be one of the major tools in these civilising projects, as illustrated by the similarities between policies directed towards the Aboriginal and Torres Strait Islander peoples in Australia, and the Sámi population in Northern Norway (Simpson and Wigglesworth 2008; Johansen 2013). In both contexts, assimilatory policies were the explicit goal of the national authorities, and schools and education were key tools in these processes. Indigenous children were sent to boarding schools where the use of their mother tongues was forbidden, with these schools seen as tools for providing cultured education and assimilating Indigenous children into the national cultures and language.

In this chapter we review this terrible history, which illustrates the many similarities in coloniser behaviours despite being at opposite ends of the world. We compare the treatment of the Australian Indigenous population with that of the Sámi in Norway, with a particular focus on the role of schools and educational systems. We end the chapter with a discussion of the current-day situation, with a focus on language reclamation work, changes that have taken place in the educational systems, and the current efforts to revitalise these Indigenous languages. Europe did not only colonise other continents but also developed and implemented tools of colonialisation at home (Lane in press). Our comparison of Australia and Norway 
reveals that, despite the different geographical situations, the processes and outcomes were remarkably similar in both cases.

\section{Historical Setting and the Linguistic Context}

\subsection{Australia}

It is estimated that the Indigenous population (the term Indigenous is used to refer to both Aboriginal and Torres Strait Islander peoples) of Australia had been the sole residents of this huge nation for a long period, between 50,000 and 120,000 years (Dudgeon et al. 2010). It is difficult to estimate the exact size of the Indigenous population prior to 1788 , but many scholars now accept that there were at least 750,000 people (Commonwealth of Australia 1998). Tragically, the period from 1790 for many decades marked a horrific period of the massacre of Indigenous peoples, including men, women, and children, by British soldiers, police, and settlers whose access to guns and other weapons, unavailable to the Indigenous population, led to extraordinary losses of Indigenous life. In 1869, the Victorian government introduced the Aboriginal Protection Act with the goal of Aboriginal "protection", but which in reality prescribed almost every aspect of Aboriginal life - including where they could live, how they could work, and whom they could marry. Following Federation in 1901, the other states followed suit with NSW introducing the Aborigines Protection Act in 1909 and with similar legislative acts passed by other states. These were punitive and restrictive laws which, while claiming to be "for their own good" were in fact far from it, resulting in the extensive the cultural genocide of the Indigenous population who were dispossessed from family, culture, and language (Dudgeon et al. 2010).

These laws allowed the authorities to move Aboriginal people from their traditional lands, often to missions or other lands far from those they knew, where they were treated as incompetent to manage their own lives, and were "subject to arbitrary rule by mission managers and police" (Commonwealth of Australia 1998, 10). Children were frequently removed from their families and the Bringing Them Home report (1997) details the experiences of these "stolen generations," and estimate that between 1910 and 1970 between $10 \%$ and $30 \%$ of Indigenous children were removed from their homes, their families, and their communities causing widespread devastation, desolation, and loss at both a personal and community level.

In pre-colonial Australia, there were 700-800 language varieties comprising between 250 and 300 distinct languages (Simpson and Wigglesworth 2018); at that time, it was not unusual for people to speak three or four languages from their local region. Many languages had small numbers of speakers, often not exceeding 1000 speakers, and that remains the case today. The impact of colonialism on these small languages was disastrous, and the decline in the number of Indigenous languages that continue to be 


\section{Pia Lane and Gillian Wigglesworth}

spoken across all generations (a necessary condition for language transmission) has been rapid. The period since colonisation has seen many traditional languages disappear, particularly in the more populated south, but at the same time there has been an increase in the number of "new" mixed languages, for example, Light Warlpiri (O'Shannessy 2012) and Gurindji Kriol (Meakins 2014). A variety of creoles have also emerged, often referred to in Australia as Kriol, with multiple dialects, which are spoken widely across the top end of Australia. In addition, there are various dialects of Aboriginal English spoken widely across the country, often acting as a lingua franca between different groups. Three National Indigenous Language Surveys have been undertaken. The first was reported in 2005 (NILS1 Australian Institute of Aboriginal and Torres Strait Islander Studies, and Federation of Aboriginal and Torres Strait Islander Languages), the second in 2014 (NILS2 Marmion, Obata and Troy 2014), and the third in 2020 (NILS3). These report that increasingly fewer children are learning their traditional language as a first language, with the number of communities where the traditional language is transmitted to children reducing from 18 in 2005 to 12 in 2020. McConvell and Thieberger (2001) predicted that by 2050 no traditional languages will still be spoken. At this rate, this prediction will come true.

Australian Indigenous languages appear genealogically unconnected to languages outside Australia, but they can be categorised into either a member of the large Pama Nyungan group (Bowern and Koch 2004), or as non-Pama-Nyungan (Evans 2003) for a small set of languages that do not meet the Pama Nyungan criteria. Typologically, Australian languages are quite similar to each other in their sound systems, the way information is structured, and the semantic categories they use (Simpson and Wigglesworth 2018). But many also have typologically rare properties, which are generally not found outside Australian languages. Kinship relations, for example, have been reported to be encoded in the syntax and morphology of the languages themselves (see, for example, Blythe 2013; Evans 2007; Hercus and White 1973). If this loss of language continues at this rate, these typological differences will be lost forever; their loss may have implications for our understanding of human cognition and its range of complexity (Evans and Levinson 2009, 431). Across the world, many Indigenous communities are experiencing this loss, a process that also affects self-perception, identity, and sense of belonging.

\subsection{Norway}

As in the case of Indigenous languages in Australia, the Indigenous Sámi languages in Europe also are in a vulnerable position. The Sámi people live in the Northern parts of Norway, Finland, and Sweden, and on the Kola Peninsula in Northwest Russia where they settled before the national borders were drawn. There are ten Sámi languages running in West-East belts, 
divided by national borders, and the result is that each Sámi language is spoken in different countries. The Sámi languages belong to the Finno-Ugric language family and are related to the national languages Finnish, Estonian, and Hungarian.

North Sámi is the largest Sámi language and is spoken in Northern Finland, Norway, and Sweden - traditionally a diverse and multilingual region and where the presence of Sámi peoples dates back for as long as we have written records. The earliest written records of this historical multilingualism are from the late ninth century when the chieftain Ottar described the Sámi livelihood as being based on hunting and fishing. Their produce was valuable and led local kingdoms to compete for jurisdiction and taxation rights, and the Sámis were regarded as the states' common subjects. In periods until 1751 when Denmark-Norway gained jurisdiction over most of the area, the Sámis paid taxes to several kingdoms, and by staking claims in this area, states strived to secure their taxation rights. When the border between Denmark-Norway and Sweden-Finland was established, the Lapp Codicil, a set of regulations regarding the rights of the border-crossing Sámi was implemented. For a fee, the Sámi were granted access to renewable resources on both sides of the border; they purchased the right to choose which country they were citizens of; rules to facilitate reindeer grazing across borders were put in place. The final border demarcation (Norway-Sweden and Finland-Russia) was established in 1826.

In Norway today, there are five main groups of Sámi peoples: Lule, Northern, Pite, Skolt, and South Sámi. Today, Pite Sámi and Skolt Sámi languages are no longer transmitted in the family domain in Norway, but there are attempts at revitalising the languages via cross-border collaboration (Todal 2020). In the Action Plan for Sámi Languages, the Norwegian Ministry of Labour and Social Inclusion (2009) estimated that in Norway less than a thousand people speak Lule and South Sámi, whereas North Sámi has approximately 25,000 speakers. In 1990, Norway ratified the ILO Convention 169 Indigenous and Tribal Peoples in Independent Countries, which defines Indigenous people as

peoples in independent countries who are regarded as Indigenous on account of their descent from the populations which inhabited the country, or a geographical region to which the country belongs, at the time of conquest or colonisation or the establishment of present state boundaries and who, irrespective of their legal status, retain some or all of their own social, economic, cultural and political institutions

Finland, Sweden, and Russia have not ratified the ILO Convention, but still regard the Sámi as an Indigenous people. Norway, Sweden, and Finland have also ratified the European Charter for Regional or Minority Languages, a convention under the auspices of the Council of Europe. The aim of the 


\section{Pia Lane and Gillian Wigglesworth}

Charter is to protect and promote languages used by traditional minorities and enable their speakers to use them both in private and public life.

\section{Assimilatory Processes - Civilising Missions}

The idea of a monolingual nation-state got a foothold particularly from the nineteenth century, and multilingualism and Indigenous languages were overlooked and often even oppressed. Dorian (1998, 10), quoting Grillo (1989, 173-174), has described the Western view of minority languages as an ideology of contempt: "an integral feature of the system of linguistic stratification in Europe is an ideology of contempt: subordinate languages are despised languages," also reflected European values transferred to the Australian context. Social Darwinism did not only rank ethnicity, but also languages (often linked to race and ethnicity), which led to a hierarchisation of languages where some languages were seen as stronger, more adaptable, and more suited for rational thought than other languages. In many cases, as discussed above, the colonised Indigenous people, and, by association, their languages, were seen as inferior and their speakers were made into "Others" through policies of assimilation and oppression. This attitude was no less prevalent in the European colonies, where oppressive policies, such as in Australia, were developed and implemented on a far larger scale.

\subsection{Australia}

The assimilationist policy adopted in Australia between 1930 and 1969 followed the earlier protectionist policy, which had been intended to reduce the Aboriginal population. In fact, the result had been a decrease in numbers of full blood Aboriginals, but an increase in the numbers of "mixed" blood or "half-caste" Aboriginals, which was taken as indicating that "Aboriginal identity could be destroyed through a process of absorption" (Armitage $1995,19)$ with the goal of complete assimilation into the white world. In 1937, all states adopted assimilation policies designed to ensure that Aboriginal people were adopting the ways of the white population with a goal of national homogeneity but also as a result of fears about the future of the white nation and the undesirability of Aboriginality in a white Australia (Moran 2005).

Children were seen in particular as being susceptible to being assimilated into Australian (European) society and they continued to be forcibly removed from their families, their homes, and their communities to be raised in missions, in boarding schools where they could be trained as domestic servants or farm labour, or placed with (largely white) foster families where, as Read $(1998,10)$ argues, they had variable experiences in their treatment, meeting dedicated foster parents, neglect, and even abuse.

The approach to assimilation inevitably varied from one state to another, including the Northern Territory, but was focussed generally on training and 
employment and education. Aboriginal families were housed in white areas; children continued to be removed and the only route to being exempted from these "protections" and controls was achieved by the Aboriginal people giving up their traditions and culture, and demonstrating that they could manage economically in white society (Moran 2005).

From the earliest days of colonisation, the Indigenous people of Australia never had their ways of life, or their traditional cultures, recognised; the protectionist policies put in place by successive state governments ensured that they had few rights. Australian Indigenous people were not recognised as Australian citizens until 1967, following a referendum in which over $90 \%$ of the enrolled population voted for changes to the constitution that would recognise Indigenous citizenship, improve services to Indigenous Australians, and allow them to be counted in future censuses (Attwood 2007). While assimilationist policies purported to be in the best interests of the Indigenous population, they had a disastrous effect on the maintenance of Indigenous languages and cultures and contributed massively to the dispossession felt widely across Australia by Indigenous communities. Indigenous Australians are still not recognised or mentioned in the Australian constitution, although this is currently under discussion, with the current Federal government announcing in 2019 that it would hold a referendum on Indigenous recognition in the constitution within three years (New York Times 2019).

\subsection{Norway}

During the eighteenth century, there was some recognition of Sámi ways of life and traditions, as they were seen as a "nation" particularly with regards to reindeer herding and in the judicial system, and Sámi was also used as a language of instruction in the educational system. In 1814, after the Napoleonic wars, Norway was ceded from Denmark to Sweden. Though in union with Sweden, Norway had its own constitution, and in this period witnessed the birth of the idea of Norway as a nation. From the mid-nineteenth century, Norway's attitude to its Northern minorities changed, and the view of the Norwegian nation came to be characterised by a drive towards a homogenous and monolingual nation. From seeing the minorities in the North as taxable subjects and a source of income, the focus shifted towards a view of diversity as problematic, and the goal became to Norwegianise the Northern minorities. The efforts to Norwegianise the minorities were fuelled by a combination of a nation-building process steeped in the idea of a homogenous nation, a fear that Russia would use the Northern minorities to lay claims to the Northern region, and a strive to "civilise" the minorities, in particular the Sámis. A range of measures were introduced, most of which targeted language and culture, aiming to assimilate the Sámi into Norwegian mainstream national culture, which was seen as more superior and civilised. Social Darwinism had a strong influence both in Norway and 


\section{Pia Lane and Gillian Wigglesworth}

Sweden, and "primitive peoples" such as the Sámis were placed at the bottom of the hierarchy.

Craniums were measured to determine what hierarchical category Sámis belonged to, and Sámi graves were opened for skulls to be moved to the University of Oslo where they could be measured and studied. Land could be given to farmers of unmixed Norwegian origin (Bull 2014, 74), and a regulation of the Land Sales Act of 1902 limited sales of land in Finnmark county to Norwegian citizens who could read, write, and speak Norwegian and who used Norwegian as their everyday language. Norwegian farmers were seen as more civilised and higher in the cultural hierarchy than the Sámis, as many Sámis were semi-nomadic, herding reindeer. Neither did they have a tradition of farming or owning land, but they had usage-based rights to areas, and therefore Norwegians settled on what they classified as free land. The goal was to establish a strong Norwegian presence in this border area and Norwegianise the population; and thus, the combination of language skills and land rights became an important tool for regulating access to land. The Act did not take into consideration traditional usage of land by groups in the area, but formalised national ownership: the land was not seen as owned by those who used it, but by the nation-state, which could redistribute land to its most loyal or appropriate subjects. Such a lack of recognition of historical use and traditional rules of land tenure is common also in other parts of the world as national states or colonisers lay claims to land (see Clifford (2013) and Connell (2007) for further examples and discussions).

\section{The Role of Educational Systems}

In many Indigenous contexts, education became the cornerstone for the development and implementation of colonising policies and led to many Indigenous peoples internalising a feeling of otherness. Such policies are found across the world, and the Kenyan writer and academic Ngũgĩ wa Thiong'o $(1986,16)$ reflects on domination through control of people's culture and self-definition in the following manner:

Its most important area of domination was the mental universe of the colonised, the control, through culture, of how people perceived themselves and their relationship to the world. Economic and political control can never be complete or effective without mental control. To control a people's culture is to control their tools of self-definition in relationship to others.

Ngũgĩ sees language as the key vehicle in this process, describing the bullet as the means of physical subjugation and language as the means of spiritual subjugation (1986, 9). Indigenous peoples, and their cultures and languages, have been vulnerable to assimilation, dispossession, and state violence, the 
latter including symbolic violence whereby "power has been legitimized in meanings institutionally produced and reproduced through everyday practices of negative stereotyping, derogatory labelling, and the devaluing of Indigenous languages and epistemologies" (Patrick 2012, 31). Both in the Australian and Norwegian contexts, schools and educational systems were tools for colonisation processes and one of the key driving forces of the subsequent language shift that occurred. Devaluation of Indigenous languages and cultures led to feelings of inferiority by those who experienced the oppressive policies, and in turn they passed their internalised worldview on to their children and grandchildren, believing that they acted in their children's best interests as knowledge of the national language was the key to success and prosperity, and for many also a way of erasing one's background to avoid further stigmatisation. Often they are not even aware of this, as time erases the memory of learned practice, and therefore social practices deeply steeped in power relations get (re)produced so subtly that social actors are not aware of these processes (see Li Wei and Kelly-Holmes' (2021) discussion of intergenerational communication as a key site for language policy and management considerations). Schools are key arenas for symbolic domination and reproduction of culture (Bourdieu and Passeron 1970) and contribute to social and cultural reproduction because knowledge in the educational system is not universal, but rather produced and shaped by particular social actors.). Boarding schools also led to separation from the family and in many cases this caused a disjuncture between the children's lives in educational settings and their home environment, which in turn for many led to a sense of alienation and loss. Such experiences are all too common, both in the Australian and in the Norwegian contexts.

\subsection{Australia}

The invasion of Australia by the British in 1788 saw the introduction of educational systems across the colony, which reflected the knowledge, values, and beliefs of Western societies. Herbert (2012) argues the for the most part this meant that Indigenous perspectives - their knowledge, their values, and their beliefs - were relegated to the periphery. Educational, employment, and social influences moved in this same direction so that not only were the Indigenous population forced to abandon their own languages, but they were also made to feel ashamed of their languages. Crucially this affected the transmission of language from parent to child with the result that, today, few Traditional Indigenous languages continue to be learned by children, although new mixed languages, English-lexified creoles (Kriol), and Aboriginal Englishes (specific dialects of English) are learned by children. Those languages where greater intergenerational transmission has been maintained are generally those where contact between the Aboriginal people and the colonisers was less, resulting in an increased presence of these languages in communities in the more remote regions of Australia 


\section{Pia Lane and Gillian Wigglesworth}

(Walsh 2005). Herbert (2012) points out that this situation persisted throughout the 1960s with a range of excuses used to explain the poor educational outcomes for the Indigenous population, which included lack of English language, poor health, low self-esteem, and cultural deprivation. Thus, rather than blame the racist nature of society, "attention could be turned away from discrimination and structured inequality as the real cause of educational disadvantage" (Lippman 1994, 138 cited in Herbert 2012), ensuring that these results could not be blamed on either the school context or on white society.

After two centuries of determined assimilationism, and with the election of the Whitlam government in 1972, the Federal government declared Indigenous languages and cultures needed to be preserved and argued for the provision of bilingual schools (Rhydwen 2007).

During the 1980s and 1990s, the modest amount of Federal government support for bilingual education for Indigenous children increased. This was a time of optimism for the future of Indigenous languages, which resulted from both Australian and international initiatives. While only a small number of Indigenous schools were established during this period, the result was an increased awareness of Indigenous issues across the country. This was reflected in various ways - through increased media coverage, but also more holistically by ensuring an increase in the presence of Indigenous history in the school curriculum, which until this point had been quite limited. In addition to this, there was much more involvement by Indigenous communities who were encouraged to participate in their children's education at school, although the support from local authorities was not universally as positive as it could have been. This support for bilingual education was reflected in an increasing number of research projects (at various institutions, but mainly in the universities), which examined in greater depth the situation of Indigenous languages across the country. Programmes were launched to support these languages through Regional Indigenous Language Centers, funded externally and managed by local Indigenous people. It was this social and educational context during the 1990s that allowed the number of bilingual programmes in English and Indigenous languages to grow to 33 in schools across Australia: 21 in the Northern Territory, 8 in South Australia, and 4 in Western Australia (Black 2007). Most of these programmes adopted a step bilingual model in which instruction began in the Indigenous language of the students, with English introduced orally, and English gradually increasing as the children advanced through the years with a transition to literacy in year four. However, despite the initial period of building and promoting Indigenous languages and culture, toward the end of the 1990s, when bilingual education in Indigenous languages that had been developed in various states, and especially in the Northern Territory, successive governments began its dismantling.

In 1998, the government of the Northern Territory, which had the largest number of bilingual programmes, decided to close them after the publication 
of a report commissioned by the administration to examine the effects of bilingual education. The reason given for these closures was the low standard of English that resulted from the bilingual programmes when compared with the standard reached by Indigenous students in school where English was the language of instruction (Nicholls 2005). However, no evidence was provided which would allow comparisons and the administration did not make public any statistics or studies that supported the claims being made about performance in English. When called upon to release the data that confirmed these assumptions, the Education Minister declined.

The final death knell for bilingual education came with the introduction of standardised testing across Australia in 2008 when the results of the National Assessment - Literacy and Numeracy Proficiency was introduced with the results of the first year demonstrating that Indigenous students did worse than non-Indigenous students, and the more remotely they lived, the lower their scores. These results were sufficient for the Northern Territory government to decide to effectively eliminate bilingual programmes by requiring the first four hours of the school day to be devoted to literacy education in English, a decision which caused many of the bilingual materials to be discarded, although the ruling was later withdrawn. There have been a few bilingual programmes that have survived in this climate, and although a few more are emerging as time passes, the vast majority of school educational programmes are still in English. Even when the only children attending these schools are Indigenous, and do not speak English either at home or in the community, when they enter school their whole schooling will be in English, although some schools do have an hour or so of language and culture each week.

\subsection{Norway}

Schools can be a powerful means for domination and subjugation, and in the mission to "civilise" and Norwegianise the Sámi population, from about 1880, the Norwegian state-issued several acts and instructions promoting Norwegian, and more importantly limiting the use of Northern minority languages. The area was multilingual, both on the societal and individual levels. Several Sámi languages and Kven, a Finnic minority language, were spoken in the area, and at least receptive competence was common for Sámi and Kven people. The aim of these policies was to ensure that Sámi and Kven children would become Norwegian speakers, and stringent measures were implemented: the possibilities for using Sámi and Kven as supportive languages in the education were removed, teachers who could document that they had been successful in this respect could apply for a salary increase, and teachers from Southern Norway were recruited (Minde 2005). The explicit goal of these policies was to "make the Sámi as Norwegian as possible - in language, culture, and in their overall view of themselves" (Todal 1998, 357). Teachers should be of "pure Norwegian descent" and 


\section{Pia Lane and Gillian Wigglesworth}

not "foreign" (Jensen 2005). Boarding schools and churches were built to demarcate the border to Russia, which was seen as a threat, and also to establish a Norwegian presence and promote Norwegian national culture in the North.

After World War II, there was a gradual change in Norwegian policies towards the minorities in the North. During the War, the Northern region had been devastated and had to be completely rebuilt after the war. The development of the Norwegian welfare state accelerated, and the authorities expected minorities to develop and be absorbed into the modern mainstream as they got to partake in what was seen as progress, modernity, and social security. The official minority language policies started to change, and a major political decision signalling this change was the Educational Act of 1959, allowing Kven and Sámi to be used in schools. At least in principle, this was the case, but in practice, the old policies lingered, and many teachers still discouraged speaking Sámi.

Somewhat paradoxically, the onset of language shift started after the oppressive policies had been lifted, but as outlined by Lane (2010), it takes time for large-scale discourses such as the language policies to be internalised and later materialised in action through language choice. The combination of negative representations and assimilation policies affected Indigenous populations' view of their language and culture and had a profound impact on language transmission in the home. Language shift may be a direct consequence of disruptive and oppressive policies, for instance, by physically relocating Indigenous children to boarding schools, or be a more indirect consequence of assimilatory policies such as when parents become convinced that giving up their mother tongue is what is best for their children (Lane 2010). Fishman (1991) emphasises intergenerational language transmission as one of the most important factors in language maintenance and in reversing language shift, but both language shift and revitalisation efforts are very complex processes that may lead to (perceived or actual) differences in competence. Consequences of language shift are often manifested in differences in communicative competence, as well as in language preferences and attitudes, particularly across generations. Elders may express sentiments of discontinuity and loss, which can contribute to the estrangement of the youth who do not speak the ancestral language, but youth may have receptive linguistic skills allowing them to continue to participate in their communities (Lane and Makihara 2017). When people of Sámi background talk about why they did not speak their Indigenous language when growing up, such reflections illustrate that language choice can be internalised so subtly that one does not really see what is happening. Rasmussen and Nolan (2011) describe this outcome as an almost unconscious, collective language choice. Such unawareness of an ongoing language shift is not uncommon and has been described by Kulick (1992) and Gafaranga (2010). Gafaranga (p. 242) reminds us that while Fishman (1991) underscores the central role of 
families and community level actions, we face a challenge because "in situations of language shift, the people involved are usually not aware of the actual interactional processes through which the shift is proceeding." Kulick $(1992,257)$ describes the process as so subtle and complex that people are not really conscious of what is happening. Children, though unaware of this process, get shaped by socialisation processes and interactional orders, and as pointed out by Meek $(2007,23)$, their "rejection of their heritage language is but one possible consequence of how children themselves engage with their sociolinguistic environments." In this sense, language shift is a result of a chain of actions, embedded in ideologies that the social actors themselves may not be aware of, and often only manage to articulate in retrospect.

A key moment in Sámi history that came to redefine the position of the Sámi people in Norway occurred in 1979 when the Norwegian Parliament voted to allow for the construction of a hydroelectric power plant in Sámi reindeer herding areas. This ignited demonstrations and hunger strikes where Sámis, environmental activists, and other supporters tried to stop the project. They did not succeed, but this event was a watershed for Sámi self-perception and claims to indigeneity. Also inspired by claims to selfsovereignty and linguistic rights by Indigenous communities internationally, the Sámi people started claiming recognition as an Indigenous people, culminating in Norway's ratification of the ILO Convention. In 1989 the Sámi Parliament was established, after the Norwegian Parliament had passed an amendment to the Norwegian Constitution committing to ensuring that the Sámi people could secure and develop their language, culture and way of life, enshrined in the Sámi Act (1987). An amendment to the Act in 1992, states that Norwegian and Sámi are equal languages and ensuring that a minimum of services in the public administration should be offered in Sámi. The proposal for a Language Act (2020) reiterates the position of Sámi and underscores the state's responsibility to protect and promote the Sámi languages in Norway.

The role of the schools changed, and from being tools of cultural domination and assimilation, schools came to be seen as central arenas for the revitalisation of the Sámi languages. Introductory courses in Sámi were introduced in two primary schools in 1967, in 1975 Sámi school districts with teaching in or of Sámi were established, and in 1989 teacher training in Sámi was offered at Sámi allaskuvla - Sámi University College, which later became the Sámi University of Applied Sciences (SUAS), today offering a wide range of subjects taught in North Sámi. Their vision is to become a Sámi University and Indigenous University, where Sámi is not only heard and written daily, but also to be a space where values and thinking of Sámi and other Indigenous peoples are in focus and contribute to scientific knowledge locally, nationally, and internationally (Thingnes 2020). This illustrates a point made by Hornberger and De Korne (2018) when they discuss how educators and educational institutions may have a positive impact 


\section{Pia Lane and Gillian Wigglesworth}

on the vitality of endangered languages and the wellbeing of endangered language speakers.

\section{Current State of Languages and Practices and Discussion of Reclamation Work}

\subsection{Australia}

Language transmission is crucial for language continuation. The situation in Australia has changed markedly over the last 230 years - many languages have been lost; many continue to be spoken but only by older generations meaning they are unlikely to continue. A few are still spoken by all generations and are the first languages of children in those communities. However, this number is rapidly declining, with five of the languages being acquired by children in 2005 no longer being learned by children in 2014 (Simpson and Wigglesworth 2018). A small number of languages, such as Anindilyakwa, Murrinhpatha, and Warlpiri, appear to have increasing numbers of speakers. At the same time, considerable language change has occurred, with several varieties of creole emerging across Australia with multiple dialects (see Simpson and Wigglesworth 2018 for a detailed discussion of the current state of languages in Australia).

In Australia, the crucial link between the Indigenous people, their land, and their languages cannot be underestimated, so language revitalisation is both important and necessary. Indigenous languages which are no longer spoken are considered to be "sleeping" (Amery and Gale 2008), so they can be reawakened using a range of resources such as old recordings, documents, and in some cases elderly speakers (Simpson 2019). The reawakening of languages has meant that many Indigenous people are now actively relearning their languages, including Kaurna (Amery 2018; Amery and Buckskin 2012), Gamilaraay (Giacon 1999), Noongar/Nyungar (Douglas 1968), Wiradjuri (Grant 2001), and Ngarrindjeri (Gale 2007). Three of these, Gamilaraay, Kaurna, and Wiradjeri, are now taught at the university level, as are Pitjantjatjara and Yolyu Matha, both languages still spoken by children (Simpson 2019). The new Australian Curriculum Framework for schoolchildren now includes three Indigenous language-learning options: the first language learner pathway, the second-language learner pathway, and the language revival learner pathway. A number of schools, both primary and secondary and including those in urban centres, now teach an Indigenous language as part of their curriculum. Bilingual educational programmes for children who speak Indigenous languages as a first language when they first come to school are also now being re-introduced in some schools. The Federal government's Indigenous Language and Arts programme provides a grant programme for community-led organisations and currently supports " 21 Indigenous Language Centres across the country working on capturing, preserving and maintaining over 150 Aboriginal 
and Torres Strait Islander languages" (Australian Government, Department of Infrastructure, Transport, Regional Development and Communications, Office for the Arts 2020). Revitalisation is thus growing with increasing levels of Federal government support through the funding of Indigenous language centres, which are often supported by linguists. There is increasing community-wide awareness of the importance of Welcomes to Country, ${ }^{1}$ with communities changing from imposed English names to traditional naming: Ernabella in South Australia, for example, is now known as Pukatja, while Santa Teresa in the Northern Territory is now called Ltyentye Apurte; these are just two examples.

\subsection{Norway}

The three Sámi languages spoken in Norway are in quite different positions today: North Sámi has the highest number of speakers; it is possible to be educated through the medium of North Sámi from kindergarten through to university level, though only municipalities that have opted to belong to the Sámi administrative district offer primary school education in Sámi, and the number of secondary schools are more limited. Education in Lule and South Sámi are considerably more limited, due to fewer speakers, lack of teachers, and more geographically dispersed populations. The Sámi Act gives stronger language rights in municipalities in the Sámi language administrative district, not only in the educational domain, but also in public administration and in the judicial system. However, a substantial part of the Sámi population live outside the administrative district, particularly in urban areas and therefore do not have the same access to education in Sámi. One of the key indicators of the position of the Sámi language in society is the number of pupils opting to study Sámi. The number of pupils studying Sámi as a second language increased and then subsequently stabilised during the period 1990-2012 (Todal 2018), which may be an indication that as a result of vitalisation efforts and the stronger position of Sámi language and culture, more families see the educational system as a possibility for their children to reclaim Sámi. However, reclaiming an Indigenous language can be a complex and contradictory process. On the one hand, it can contribute to the healing of trauma and loss from the past; on the other, through the very act of reclaiming their language, speakers are forced to face such trauma and loss. People who reclaim Sámi as adults frequently use the term språksperra ("the language barrier") to describe feelings of anxiety, reluctance to speak Sámi, and a fear of being judged by traditional speakers. Linguistic insecurity is known from other Indigenous settings. Based on their study of Ojibwe language reclamation, King and Hermes (2014) point out that emotional aspects of language learning in Indigenous contexts seem to be stronger than in second-language acquisition contexts because of identity politics and social control as to who has the right to claim the role of an authentic speaker run deeper in Indigenous settings. 


\section{Pia Lane and Gillian Wigglesworth}

The consequences and impact of assimilatory and colonialising policies are addressed by several nation-states through the establishment of Truth and Reconciliation Commissions, such as Canada and Greenland. Norway's TRC was established in 2018, and it aims to map policies and activities of the Norwegianisation process, lay the groundwork for the recognition of the consequences these experiences had for Sámi and Kven peoples and suggest measures for future reconciliations. An underlying idea is that assimilatory and colonialising policies are not only a concern for minorities and Indigenous groups, but also a national heritage and responsibility for the nation as a whole.

\section{Concluding Thoughts}

In spite of geographical distance, and demographically and sociolinguistically very different environments, these two contexts have strikingly similar historical backgrounds and similar assimilatory processes. In both Australia and Norway, assimilatory policies were the explicit goal of the national authorities, and schools and education were key tools used in controlling the Indigenous populations. Today, the role of education has undergone significant change. In Norway, schools that used to be tools of assimilatory policies have become potential arenas for the reclamation of language and culture. In the more complex Australian situation, language reclamation tends to be more community-based, although the Australian Curriculum, taught throughout the country, now offers Indigenous languages as an option for children right through the school curriculum and Indigenous culture and history is much more integrated into all areas of the school curriculum than it was. We have shown how educational systems both in Australia and Norway were key tools for colonialism and nation-state building that devalued and ignored Indigenous languages, practices, and ways of knowing. McKinley and Tuhiwai Smith (2019, 2 ) remind us that Indigenous groups have always had complex education systems, but colonialism and exploitation have shattered Indigenous knowledges and ways of knowing. Today, schools may have the potential for healing pain and restoring disruptions caused by oppressive policies. However, schools can be more than a domain for the teaching of or in Indigenous languages; they can also be a space for acknowledging Indigenous ways of knowing, learning, and teaching in critical engagement with Western tradition.

Indigenous languages have suffered immensely from Western views of them as inferior, but in many cases, as demonstrated in this chapter, increasing efforts are being made at revitalising them. The most critical factor in maintaining languages is and will always be language transmission through the generations. As Curdt-Christiansen and Lanza (2018) point out, this involves not only explicit and deliberate decisions about language use in the home, but also reflects the unconscious and implicit decisions that parents make in the way they engage with language with their children, and the 
way children themselves engage with their language input as argued so eloquently by Lanza (1997). Language choices and practices both within the family and in the educational systems, are shaped by explicit language policy, and by internalisation of values resulting from linguistics stratification. Therefore, current language practices in families and schools show consequences of assimilatory processes. In both contexts we have investigated, we also see the possibility of revitalisation efforts to reverse some of these negative effects and bring a measure of healing as Indigenous languages, cultural practices and ways of knowing are reclaimed. These languages and epistemologies are not things from the past, but as Linda Tuhiwai Smith (2012) reminds us, reclaiming a voice is a matter of reclaiming, reconnecting and reordering Indigenous ways of knowing.

\section{Acknowledgements}

This chapter was partly financed by the Research Council of Norway through its Centres of Excellence funding scheme, project number 223265 to the first author, and through the Australian Research Council through its Centres of Excellence funding scheme, number CE14001000041 to the second author.

\section{Note}

1 This is a ceremony which is performed by the Traditional Indigenous Owners of the land on which an event (e.g. a concert, lecture or artwork) is taking place.

\section{References}

Amery, Rob. 2018. "Revitalization of Kaurna." In The Routledge Handbook of Language Revitalization, edited by Leanne Hinton, Leena Huss, and Gerald Roche, 330-341. New York, NY: Routledge.

Amery, Rob and Vincent Kanya Buckskin. 2012. "Handing on the Teaching of Kaurna Language to Kaurna Youth.” Australian Aboriginal Studies 2: 31-41.

Amery, Rob and Mary-Anne Gale. 2008. "But Our Language was Just Asleep: A History of Language Revival In Australia." In Encountering Aboriginal Languages: Studies in the History of Australian Linguistics, edited by William B. McGregor, 339-382. Canberra, ACT: Australian National University.

Armitage, Andrew. 1995. Comparing the Policy of Aboriginal Assimilation: Australia, Canada, and New Zealand. Vancouver: UBC Press.

Attwood, Bain. 2007. The 1967 Referendum: Race, Power and the Australian Constitution. Canberra: Aboriginal Studies Press.

Australian Government. 2020. Department of Infrastructure, Transport, Regional Development and Communications, Office for the Arts, 2020. https://www.arts. gov.au/what-we-do/Indigenous-arts-and-languages/Indigenous-languages-andarts-program.

Black, Peter. 2007. "New Uses for Old Languages." In Language and Culture in Indigenous Australia, edited by Michael Walsh, and Colin Yallop, 207-223. Canberra: Indigenous Studies Press. 
Blythe, Joe. 2013. "Preference Organization Driving Structuration: Evidence from Australian Aboriginal Interaction for Pragmatically Motivated Grammaticalization." Language 89 (4): 883-919.

Bowern, Claire, and Harold Koch, eds. 2004. Australian Languages: Classification and the Comparative Method. Philadelphia/Amsterdam: John Benjamins.

Bringing them Home. 1997. Report of the National Inquiry into the Separation of Aboriginal and Torres Strait Islander Children from Their Families. Human Rights and Equal Opportunity Commission. Canberra: Government of Australia.

Bourdieu, Pierre, and Jean-Claude Passeron. 1970. La Reproduction. Paris: Les Editions De Minuit.

Bull, Kirsti Strøm. 2014. Jordsalgslovgivning. En Rettshistorisk Lovgjennomgang av Jordsalgslovgivningen i Finnmark $i$ Perioden 1775-1965. https://www.dom stol.no/globalassets/upload/finn/sakkyndige-utredninger/jordsalgslovgivningenkirsti-strom-bull.pdf.

Clifford, James. 2013. Returns. Becoming Indigenous in the Twenty-First Century. Cambridge, Mass: Harvard University Press.

Commonwealth of Australia. 1998. As a Matter of Fact: Answering the Myths and Misconceptions about Indigenous Australians. Canberra: ATSIC.

Connell, Raewyn. 2007. Southern Theory: The Global Dynamics of Knowledge in Social Science. Cambridge: Polity.

Curdt-Christiansen, X.L., and Elizabeth Lanza. 2018. "Language Management in Multilingual Families: Efforts, Measures and Challenges." Multilingua 37(2): 123-130.

Dorian, Nancy C. 1998. "Western Language Ideologies and Small-Language Prospects.” In Endangered languages: Language Loss and Community Response, edited by Lenore A. Grenoble and Lindsay J. Whaley, 3-21. Cambridge: Cambridge University Press, 1998.

Douglas, Wilfrid. 1968. The Aboriginal Languages of the South-West of Australia. Canberra: AIAS.

Dudgeon, Pat, Michael Wright, Yin Paradies, Darren Garvey, and Iain Walker. 2010. "The Social, Cultural and Historical Context of Aboriginal and Torres Strait Islander Australians." In Working Together: Aboriginal and Torres Strait Islander Mental Health and Wellbeing Principles and Practice, edited by N. Purdie, P. Dudgeon, and R. Walker, 25-42. Barton ACT: Australian Government Department of Health and Ageing.

Evans, Nicholas. 2003. The Non-Pama-Nyungan languages of Northern Australia: Comparative Studies of the Continent's Most Linguistically Complex Region. Canberra: Pacific Linguistics.

Evans, Nicholas. 2007. "Warramurrungunji Undone: Australian Languages in the 51st Millennium." In Language Diversity Endangered, edited by Matthias Brenzinger, 342-373. Berlin: Mouton de Gruyter.

Evans, Nicholas and Stephen C. Levinson. 2009. "The Myth of Language Universals: Language Diversity and its Importance for Cognitive Science." Behavioral and Brain Sciences 32: 429-448.

Fishman, Joshua. 1991. Reversing Language Shift: Theoretical and Empirical Foundations of Assistance to Threatened Languages. Clevedon: Multilingual Matters.

Gale, Mary-Anne. 2007. Ngarrindjeri Learners' Guide (Trial Edition). Adelaide: Raukkan Community Council on behalf of the Ngarrindjeri Community. 
Gafaranga, Joseph. 2010. “Medium Request: Talking Language Shift into Being.” Language in Society 39 (2): 241-270.

Giacon, John. 1999. Yaama Maliyaa, Yuwaalaraay - Gamilaraay: An Aboriginal Languages Textbook. Walgett, NSW: Walgett High School, Yuwaalaraay Gamilaraay Program.

Grant, Stan. 2001. Wiradjuri Language: How It Works: A Grammar in Everyday English. Canberra: Restoration House.

Grillo, Ralph. D. 1989. Dominant Languages: Language and Hierarchy in Britain and France. Cambridge: Cambridge University Press.

Herbert, Jeannie. 2012. "Ceaselessly Circling the Centre: Historical Contextualization of Indigenous Education within Australia.” History of Education Review 41 (2): 91-103.

Hercus, Luise A. and Isobel M. White. 1973. "Perception of Kinship Structure Reflected in the Adnjamathanha Pronouns." Papers in Australian Linguistics 6: 47-75. Canberra: Pacific Linguistics.

Hornberger, Nancy and Haley De Korne. 2018. "Is Revitalization Through Education Possible?" In The Routledge Handbook of Language Revitalization, edited by Leanne Hinton, Leena Huss, and Gerald Roche, 94-103. New York: Routledge.

Jensen, Eivind Bråstad 2005. Skoleverket og De Tre Stammers Møte. Tromsø: Eureka.

Johansen, Åse. 2013. “Overcoming Silence: Language Emancipation in a Coastal Sámi-Norwegian Community.” Sociolinguistic Studies 7 (1-2): 57-77.

King, Kendall and Mary Hermes. 2014. "Why is This So Hard?: Ideologies of Endangerment, Passive Language Learning Approaches, and Ojibwe in the United States." Journal of Language Identity and Education 13 (4): 268-282.

Kulick, Don. 1992. Language Shift and Cultural Reproduction: Socialization, Self, and Syncretism in a Papua New Guinean Village. Cambridge: Cambridge University Press.

Lane, Pia (in press) "The South in the North: Colonialisation and Decolonisation of the Mind." In From Southern Theory to Decolonizing Sociolinguistics - Voices, Questions and Alternatives, edited by Deumert, Ana and Sinfree Makoni. Multilingual Matters.

Lane, Pia. 2010. “'We Did What We Thought was Best for Our Children.' A Nexus Analysis of Language Shift." International Journal of the Sociology of Language 202: 63-78.

Lane, Pia and Miki Makihara. 2017. "Indigenous Peoples and Their Languages." In The Oxford Handbook of Language and Society, edited by García, Ofelia, Nelson Flores, and Massimiliano Spotti, 299-320. Oxford: Oxford University Press.

Lanza, Elizabeth 1997. Language Mixing in Bilingual Children. New York: Oxford University Press.

Li Wei and Helen Kelly-Holmes. 2021. "Language Policy: Taking Stock in a Changing Context." In Spaces of Multilingualism, edited by R. Blackwood and U. Røyneland. London, New York: Routledge.

Marmion, Doug, Kazuko Obata, and Jakelin Troy. 2014. Community, Identity, Wellbeing: The Report of the Second National Indigenous Languages Survey. Canberra: Australian Institute of Aboriginal and Torres Strait Islander Studies.

Mckinley, Elizabeth Ann and Linda Tuhiwai Smith. 2019. Handbook of Indigenous Education. Singapore: Springer Singapore. 


\section{Pia Lane and Gillian Wigglesworth}

McConvell, Patrick and Nicholas Thieberger. 2001. State of Indigenous Languages in Australia -2001. Australia State of the Environment Technical Paper Series (Natural and Cultural Heritage), Series 2. Canberra: Department of the Environment and Heritage.

Meakins, Felicity. 2014. "Language Contact Varieties." In The Languages and Linguistics of Australia: A Comprehensive Guide, edited by Harold Koch and Rachel Nordlinger, 365-416. Berlin: Walter de Gruyter.

Meek, Barbra. 2007. "Respecting the Language of Elders: Ideological Shift and Linguistic Discontinuity in a Northern Athapascan Community." Journal of Linguistic Anthropology 17 (1): 23-43.

Minde, Henry. 2005. "Fornorskinga av Samene - Hvorfor, Hvordan og Hvilke Følger?/ Sámiid Dáruiduhttin - Manin, Mo ja Makkár Váikkuhusat?” In Samisk Skolehistorie/Sámi Skuvlahistorjá, edited by Svein Lund, 12-33. Kárášjohka: Davvi girj.

Norwegian Ministry of Labour and Social Inclusion. 2009. "Action Plan for Sámi Languages.” https://www.regjeringen.no/globalassets/upload/fad/vedlegg/sami/h p_2009_samisk_sprak_engelsk.pdf

Moran, Anthony. 2005. "White Australia, Settler Nationalism and Aboriginal Assimilation." Australian Journal of Politics and History 51 (2): 168-193.

National Indigenous Languages Survey Report 2005: Report Submitted to the Department of Communications, Information Technology and the Arts, 255. Canberra: DCITA.

Nicholls, Christine. 2005. "Death by a Thousand Cuts: Indigenous Language Bilingual Education Programmes in the Northern Territory of Australia, 19721998." International Journal of Bilingual Education and Bilingualism 8 (2-3): $160-177$.

O'Shannessy, Carmel. 2012. "The Role of Codeswitched Input to Children in the Origin of a New Mixed Language." Linguistics 50: 305-340.

Patrick, Donna 2012. "Indigenous Contexts." In The Routledge Handbook of Multilingualism, edited by Marilyn Martin-Jones, Adrian Blackledge, and Angela Creese, 29-48. Abingdon, UK: Routledge Press.

Rasmussen, Torkel, and Shaun Nolan. 2011. "Reclaiming Sámi Languages: Indigenous Language Emancipation from East to West." International Journal of the Sociology of Language (209): 35-55. doi:10.1515/ijs1.2011.020

Read, P. 1998. "The Return of the Stolen Generation." Journal of Australian Studies 22 (59): 8-19.

Rhydwen, Mary. 2007. "Kriol: The Creation of a Written Language and a Tool of Colonisation." In Language and Culture in Indigenous Australia, edited by Michael Walsh and Colin Yallop, 155-168. Canberra: Indigenous Studies Press.

Simpson, Jane. 2019. “The State of Australia's Indigenous languages - and How We Can Help People Speak Them More Often.” Languages Victoria 23 (1): 71.

Simpson, Jane and Gillian Wigglesworth, eds. 2008. Children's Language and Multilingualism: Indigenous Language Use at Home and School. UK, Continuum International.

Simpson, Jane and Gillian Wigglesworth. 2018. "Language Diversity in Indigenous Australia in the 21st Century." Current Issues in Language Planning 20: 67-80.

Smith, Linda Tuhiwai. 2012. Decolonizing Methodologies: Research and Indigenous peoples, 2nd ed. London: Zed Books. 
Thingnes, Jorunn Simonsen 2020. "Making Linguistic Choices at a Sámi University: Negotiating Visions and Demands." Current Issues in Language Planning 21 (2): 153-174.

Todal, Jon. 1998. "Minorities with a Minority: Language and the School in the Sami Areas of Norway." Language, Culture and Curriculum 11 (3): 354-366.

Todal, Jon. 2018. "Quantitative Changes in the Status of the Sámi Language in Norway. A Summary of Existing Knowledge." In Sami Statistics Speak. Numbers and Commentary Vol. 1/2018, edited by Bella Crespin, 87-111. Guovdageaidnu: Sámi University College - Sámi allaskuvla: Guovdageaidnu.

Todal, Jon. 2020. "Sámi." Linguistic Minorities in Europe (no pagination in article) doi:10.1515/lme.12036397

Wa Thiong'o, Ngũgĩ. 1986. Decolonising the Mind: The Politics of Language in African Literature. London: James Currey.

Walsh, Michael. 2005. "Indigenous Languages of Southeast Australia, Revitalization and the Role of Education." Australian Review of Applied Linguistics 28 (2): $1-14$. 


\section{Part III}

\section{Bilingual Ageing}

Communication and

Cognitive Impairments 


\title{
7 Where Have All My Languages Gone? Aging and the Changing Multilingual Linguistic Ecology
}

\author{
$\mathrm{Ng}$ Bee Chin and Francesco Cavallaro
}

\section{Introduction}

Effective communication is crucial in the healthcare sector and a growing body of research indicates that the language barriers encountered in healthcare settings may compromise the quality of care (Coren, Filipetto, and Weiss 2009; Schyve 2007; Williams, Weinman, and Dale 1998). This issue is exacerbated when doctors and patients do not share a common language. In the past few decades a considerable amount of research in English-speaking countries has focused on identifying effective practices for improving communication between clinical staff and patients with limited English proficiency (LEP) (Attard et al. 2013; Ngo-Metzger et al. 2007). This is particularly pertinent to Singapore's public healthcare system, which has adopted English as its main working language. However, Singapore displays a different setting to the studies in other countries (the USA, UK, Australia, New Zealand). In these countries, the focus has been on the LEP of new migrants or of migrant populations for whom English is not a mother tongue, and the encounters of these minority groups with the healthcare systems is the usual migrant story of having to integrate or to adapt to a different host culture. For this widely studied group, the issue is of language adaptation in a new land. In Singapore, the language barriers experienced by older adults is a result of the change in the multilingual language ecology resulting in these older adults being displaced as the languages they speak become irrelevant. The cause of this displacement is fundamentally different from the cases that are reported in current literature, but the impact is just as devastating if not more so as it is happening in your home country where you expect to find refuge. This language rupture is not only taking place in the healthcare sector but in the community and at home.

This chapter documents the language problems faced by older adults (OAs) not in a migration context, but in their own home. In Singapore, a significant portion of OAs either do not speak or are not proficient in any of the languages used by the healthcare providers. To this end, we will focus on medical encounters as they are emblematic of the wider social and linguistic barriers experienced by Chinese-speaking OAs in Singapore. 


\section{Limited English Proficiency and Healthcare}

Many studies in English-speaking countries have shown the negative effects of language-discordance between healthcare providers and LEP patients (Regan Freeman 2014; Capps, Rolfe, and Logsdon 2016). Ineffective communication as a result of patients' LEP has been found to worsen health disparity, reduce their access to care, and compromise in quality of care and treatment outcomes (Regan Freeman 2014). Manifestations of this include patients' infrequent visits to healthcare providers, delayed treatments, clinical errors, increased susceptibility to chronic diseases, and health complications (Coren, Filipetto, and Weiss 2009). There are also intangible interactional issues such as patient and provider dissatisfaction, as well as anxieties, misunderstandings, and uncertainties experienced by LEP patients due to the communication problems they face (Coren, Filipetto, and Weiss 2009; Regan Freeman 2014).

An obvious solution is the use of interpreters in medical encounters. "Interpreters" refer to professional interpreters employed by a healthcare institution, or ad hoc, untrained individuals, such as nurses, family members, friends, non-clinical hospital employees, and even strangers from waiting rooms. Ad hoc interpreters tend to lead to significant clinical errors such as omission and substitution of sensitive information, consequently compromising the quality of care. This is due to the fact that the role of interpretation is poorly understood by most untrained individuals (Rosenberg, Seller, and Leanza 2008). Meyer, Pawlack, and Kliche (2010) report that most family interpreters described their role as merely to facilitate understanding rather than to render exactly what was said. Some added that they restricted themselves to interpreting only what they sensed that their family member needed help in or was being misunderstood. Others admitted to not interpreting all interactions between the doctors and patients and deliberately censoring some information as and when they saw fit. Well-meaning, family interpreters can potentially create more misunderstandings or further aggravate existing communicative difficulties.

It is well attested that qualified and trained medical interpreters are significantly less likely to make clinical mistakes than family interpreters, and their use leads to an enhancement in the overall quality of care for LEP patients through a reduction in interpretation errors, increased understanding, with the resultant satisfaction of patients (Karliner et al. 2007; Flores 2005; Betancourt et al. 2012; Arthur et al. 2015). Despite these iron-clad statistics, non-professional interpreters are used worldwide to provide language assistance in many contexts (Rosenberg, Seller, and Leanza 2008; Schenker et al. 2011). As a general practice in the USA, professional interpreters are found mainly in complicated medical processes, whereas those perceived to be less complex often simply involve "getting by" with the use of ad hoc interpreters (Tang et al. 2014; Schenker et al. 2011). 
With the increasing globalization of human movement leading to increased language and cultural diversity, healthcare sectors globally face similar linguistic challenges as they encompass a significant proportion of patients who do not speak the official language(s). This issue is not confined to Singapore or Asia and, indeed, is a pressing concern to cosmopolitan cities all over the world. Despite the fact that these observations are empirically robust, the use of ad hoc interpreters persists in Singapore and in medical systems across the world.

\section{A Linguistic Snapshot of Singapore}

Singapore is multicultural, multilingual, and multireligious. Its population is made up of three major ethnic groups and a significant migrant worker population. The categorization of these three major ethnic groups in many ways obfuscates the teeming diversity in terms of mutually non-intelligible language use within each ethnic group.

Singapore's population has doubled in the last 35 years. This increase is also driven by the size of its transient workforce, which has increased from 200,000 in 1980 to $1,677,391$ or $29.4 \%$ of the total population in 2019 . These are typically manual workers from the region and also includes healthcare providers like nurses and nursing aides from the PRC, India, the Philippines, Myanmar, and Indonesia. These migrant workers, together with the 4,026,209 citizens and permanent residents, bring the current total population to 5,703,600 people (Department of Statistics 2019).

The 4.02 million Singapore citizens and residents are made up of 74.36\% Chinese, $13.43 \%$ Malays, 9.01\% Indians, and 3.21\% of Others (Department of Statistics 2019). There are four official languages, English, Mandarin, Tamil, and Malay, and Malay is also the national language. Other than the four official languages, many other languages are also spoken by Singaporeans. In the Chinese community a number of Chinese vernaculars, such as Hokkien, Cantonese, Teochew, Hakka, and Hainanese (to name the few common ones), are spoken together with Mandarin Chinese. The Indian community consists of speakers of Tamil, Hindi, Punjabi, Bengali, Malayalam, Telugu, and others, and the Malay community also include numbers of Boyanese, Javanese, and Baba Malay (Peranakan) speakers. Most Singaporeans 50 and below are bilingual in English and at least one official languages. Across ethnic groups, the common language is English, which is also sometimes the lingua franca within ethnic groups (Cavallaro, $\mathrm{Ng}$, and Tan 2020). All these communities are experiencing an ongoing language shift toward English, the language used nationwide in business, administration, law, and education. This dramatic social transformation in linguistic ecology has been extensively covered by several researchers (see Cavallaro and $\mathrm{Ng}$ 2014, 2020). A synopsis is presented in the following paragraph. 
The Singapore linguistic landscape has been forcibly shaped by two key language policies: (i) the Bilingual Policy has been emphasizing an Englishplus bilingualism since independence in 1965; and (ii) the Speak Mandarin Campaign (SMC), launched in 1979, promotes Mandarin Chinese over all other Chinese languages. The Bilingual Policy catapulted English as the premium language as it became the medium of instruction in all schools and also the de facto language for all formal contexts, despite the existence of four official languages. Since the launch of the SMC, the census data has been showing a steady increase in the population that uses English as the predominant household language. Economic success has convinced the majority of Singaporeans that a good knowledge of English is the basis for better career opportunities for themselves and their children. The widespread use of English among all ethnic groups and the majority (in numbers) of the ethnically Chinese have elevated English and Mandarin to the status of majority languages, and relegated other Chinese vernaculars, Malay, and Tamil to the rank of minority languages. This period has also seen a dramatic decline in the number of speakers of the Chinese vernaculars, who now largely comprise the OAs in the Chinese community $(\mathrm{Ng}$ and Cavallaro 2021).

A cursory look at the demographic data shows that Singaporeans who are 50 and below (born after independence in 1965) are linguistically well equipped to negotiate the Singapore of today, which requires proficiency in English for virtually all formal domains. Conversely, this same group of Singaporeans have reduced or no capacity in the vernaculars spoken by their parents or grandparents. So, what we have here is a language ecology that has been dramatically transformed over the course of five decades.

Our interest here is with the older Singaporeans. According to the Singapore Population Census, the number of residents aged 65 and above has grown from $6 \%$ in 1990 to $14.4 \%$ of the population in 2019 . In the same period, the median age has increased from 29.8 years to 41.1 (Department of Statistics 2019). The number of OAs are projected to increase to $22.1 \%$ of the population by 2030 and $34 \%$ by 2050 .

As indicated earlier, the OAs in Singapore are in many ways different from those in the UK and the USA where the LEP patients are either new migrants or migrants who have been uprooted from their source culture. This particular group of OAs in Singapore are born and bred in Singapore but are caught in an alien linguistic landscape due to sweeping and dynamic changes in language practices, mainly due to state-driven language policies as well as the forces of globalization.

These changes in language use, attitudes, and repertoire have impacted on OAs in very significant ways. First, as most OAs live with their children and grandchildren, the increase in the use of Mandarin and English in the home domain means that many of these OAs are effectively cut off from communicating directly with their grandchildren (Tan and $\mathrm{Ng} 2010$ ). Second, their lack of proficiency in the country's majority languages means 
that they are unable to interact meaningfully with the wider community. They live in a landscape that is increasingly dominated by the sights and sounds of languages they are not familiar with. They are unable to talk to their grandchildren, use the banks, manage formal transactions on their own, read public signs, and, most important of all, navigate the healthcare system, which is so vital for their well-being. Their language barriers have become a significant handicap as they are increasingly out of sync with the English-dominated new Singapore. Though it is tempting to see this group of OAs as similar to the LEP patients in the literature, they are qualitatively different as for them, the possibility of a home elsewhere does not exist.

For the OAs in Singapore, the inability to access the new language world is very akin to being a stranger in your own home. Hence, instead of referring to this group of OAs as LEP patients, we will refer to them as Linguistically Displaced Older Adults (LDOA). The lives of these LDOAs have transitioned from thriving multilingual and diverse contexts to one where linguistic pragmatism has promoted the rise of some languages to the demise of others at considerable cost to the social well-being of these LDOAs. In the next section, we will focus on medical encounters in the healthcare system as one of the realities of aging is an increased reliance on healthcare providers and medical facilities.

\section{The Healthcare System in Singapore}

An aging population in Singapore, coupled with a projection of the population's greater reliance on the public healthcare system, has brought to the forefront the importance of preparing an equipped workforce and an enhanced healthcare system geared for the future (Cavallaro et al. 2016; Gan 2012). In recent years, there has been a significant and growing proportion of foreign healthcare professionals in Singapore's public healthcare sector due to a shortage of local people joining the sector, as evidenced by the fact that more than $25 \%$ of Singapore's doctors today are foreigners (TST 2015). Coupled with a significant aging population, there is growing dissonance between the language needs of LDOAs and the language used by healthcare professionals pointing to a crucial need for greater consideration of the linguistic challenges faced by these patients. However, the official stance and that of the public hospitals in this aspect has been marked with passivity, albeit with several acknowledgments of a need for greater attention in this area (Neo 2010; Maryam Mokhtar 2014).

The approach on how to tackle these language barriers adopted by the public healthcare sector thus far is to send foreign medical staff (usually the nurses) to language courses in order for them to acquire the necessary (local) languages to converse with patients. A more common practice is to tap on the existing linguistic abilities of local staff (Maryam Mokhtar 2014). Public hospitals, in general, seem to adopt an approach of engaging interpreters only when it comes to foreign languages and not when the language of 


\section{$152 \mathrm{Ng}$ Bee Chin and Francesco Cavallaro}

concern is a local one. An exception is the National University Hospital (NUH), which hired one "dialect" interpreter in 2010, and its plan was to hire more (Neo 2010). To date, however, no other interpreter has been hired. Note that in Singapore the mutually non-intelligible Chinese vernaculars are referred to as "dialects."

To address the needs of the aging population, the Singapore Government set up the Ministerial Committee on Aging (MCA) in 2007, and their vision is "Successful Ageing for Singapore." However, while much has been done in building up the infrastructure to make all spaces more accessible to older residents, very little seems to have been directed at the more intangible aspects of accessibility in terms of the language issues faced by Singaporean LDOAs. The interesting aspect of this is that most of the people of this generation may in fact be very multilingual but are unable to speak or understand English. Their proficiency in Mandarin may also be quite poor (Tan and Ng 2010). Very sadly, most of these LDOAs find themselves surrounded by a world of unfamiliar languages at the final stages of their lives, grappling not only with a myriad of problems associated with aging as well as anxiety and trauma caused by communication barriers.

As indicated earlier, for interpretation needs, local medical institutions simply tap on their own staff who are proficient in the vernaculars (Neo 2010). The Ministry of Health assures the citizens that they roster a good mix of local and foreign healthcare staff at every shift as a measure to reduce the risk of miscommunication. With the advantage of having vernacularspeaking staff, the employment of interpreters for local languages is therefore considered unnecessary by most healthcare institutions.

This chapter will discuss the language issues of LDOAs in the context of medical encounters through a series of semi-structured interviews with LDOAs, nurses, and doctors.

\section{Methodology}

In total, 35 participants (Table 7.1) were interviewed for this study (30 older adults, 5 healthcare providers). The 30 older adults ( 8 males and 22 females) were between the ages of 66 and 83 and were in need of regular

Table 7.1 Participants

\begin{tabular}{lllll}
\hline Males & Females & Main Language & Age & Role \\
\hline 1 & 5 & Mandarin & $68-78$ & Patient \\
3 & 9 & Hokkien & $66-82$ & Patient \\
4 & 8 & Cantonese & $66-83$ & Patient \\
- & 3 & English & $29-71$ & Nurses \\
- & 2 & English & 29 & Doctors \\
\hline
\end{tabular}


medical attention as several have chronic illnesses, such as high blood pressure, prolonged joint issues leading to knee or hip replacement, or diabetes. They were recruited through the network of our research assistants and subsequently by referral sampling as participants then recommended others from their circle of connections. The five healthcare providers were three nurses and two doctors.

The data for the study came from a series of interviews obtained through face-to-face and telephone interviews. Face-to-face semi-structured interviews were conducted with the patient participants and nurses, and telephone interviews were conducted with the doctors.

Prior to each interview, the participants were briefed on the focus of the research, and their verbal informed consent was collected. The interviews with the LDOAs were conducted in Mandarin, Cantonese, and Hokkien, depending on the preferences of the participants. None of the LDOAs were able to speak English, six could speak Mandarin, and the rest only had minimal proficiency in Mandarin Chinese. The other participants reported Hokkien $(\mathrm{n}=12)$ and Cantonese $(\mathrm{n}=12)$ as the language they were most proficient in. The interviews with the nurses and doctors were conducted in English. All participants were interviewed only once and for a time ranging from 15 minutes to 30 minutes. The interviews were audio-recorded, transcribed, and then (those with the LDOAs) translated into English. Upon completion, each participant received a token of appreciation.

Three distinct sets of questions were designed for each target group. The questions to the LDOAs focused on their visits to the larger public hospitals in Singapore and their language proficiency. The questions probed their language needs, for example, whether they needed help when talking to the doctors and how they felt when faced with language barriers. For the nurses and doctors, the questions were aimed at eliciting the strategies they adopted when faced with patients who did not speak English and how they felt about these encounters. To anonymize the data, the interviewees are given a code. The participating LDOAs are referred to according to their gender ( $\mathrm{M}$ or $\mathrm{F}$ ), language $(\mathrm{C}=$ Cantonese, $\mathrm{M}=$ Mandarin, and $\mathrm{H}=$ Hokkien), and age. Therefore, a male Cantonesespeaking participant who was 76 years old would be referred to as: MC76. These identifiers were unique for each participant as no two participants had the same code.

A thematic analysis of the comments by all participants was carried out by the senior members of the research team. However, a consensus on the main themes was arrived at in collaboration with the entire research team. The analysis of the LDOAs focused on communication barriers experienced and their responses and perceptions of difficult medical encounters. The analysis of the interviews of nurses and doctors focused on their perception of their own experience of communication barriers and what resources they drew on. 


\section{$154 \mathrm{Ng}$ Bee Chin and Francesco Cavallaro}

\section{The Findings}

\subsection{Perspective From the Linguistically Displaced Older Adults}

The LDOAs interviewed reported that they were usually accompanied by at least one child during hospital visits or at times came alone. All of them had encountered healthcare professionals with and without Chinese-speaking competency.

The analysis of the transcripts revealed several recurring issues that these elderly patients encountered on their hospital visits:

- Challenges in communicating with doctors,

- Heavy reliance on ad hoc interpreters,

- High dependence on older adults' own initiative for the communication outcome, and

- Passive acceptance of and frustration by the older adults in languagediscordant medical encounters.

\subsubsection{Challenges in Communicating With Doctors}

The respondents who were always accompanied by their children in hospital visits all indicated that the reason they were unable to make these visits alone was due to the English-dominated landscape in public hospitals. By this, they were referring to signage (see the example in Figures 7.1 and 7.2)

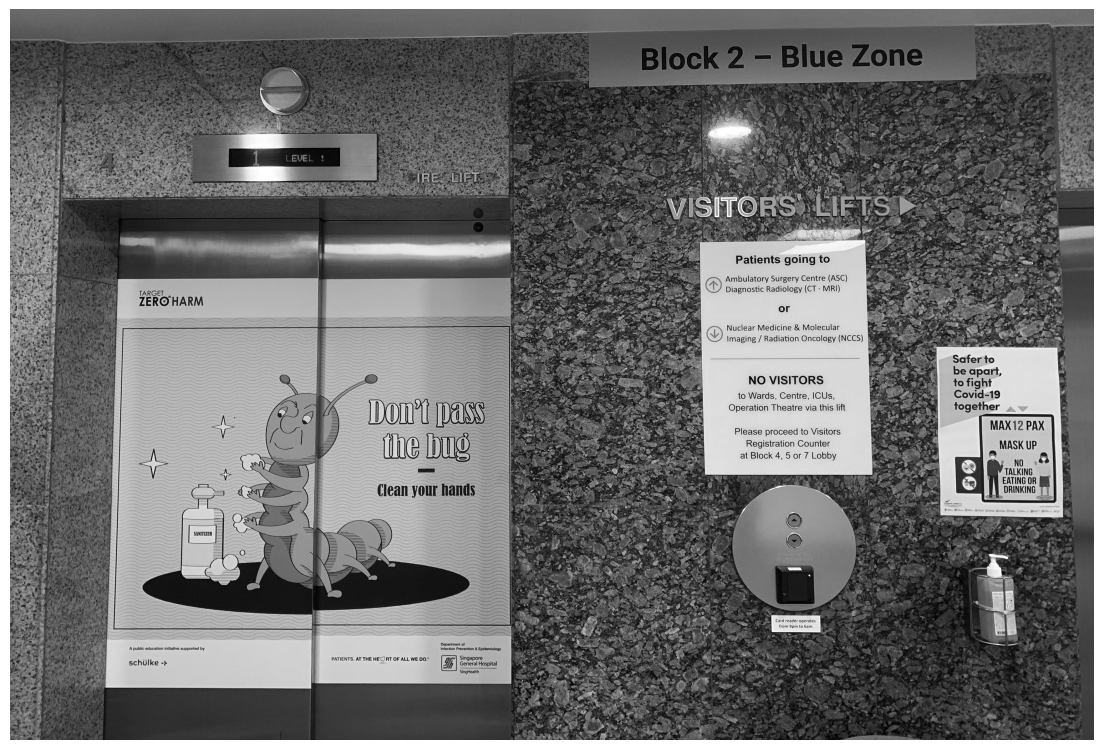

Figure 7.1 Signs outside a lift in a hospital 


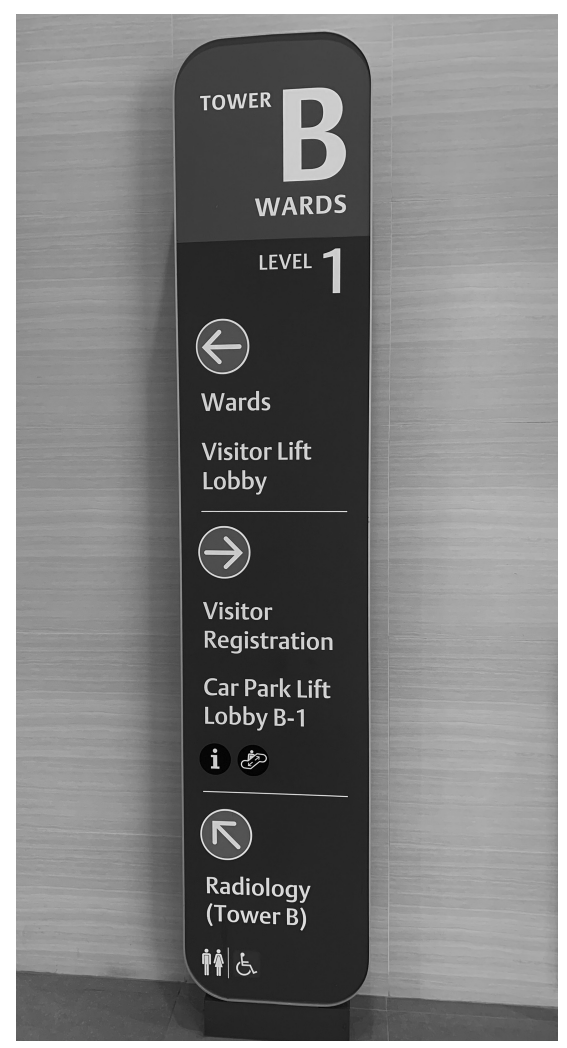

Figure 7.2 Sign at a hospital lobby

in the hospitals as well as simple things such as the announcement of queue numbers and calling out of instructions. All these sensory inputs in hospitals are in English. Many discussed the trips to the hospital as though they were talking about negotiating obstacle courses.

The linguistic landscape of Singapore unambiguously indexes English as the prime language of wider function. Most studies of linguistic landscape document linguistic vitality through the study of signs and their relationship to power and inequality (see Lanza and Woldemariam 2014), but in Singapore, the dominance of English in the landscape is so complete and absolute that the only time it is significant is when it does not dominate. This reflects the greater shift toward a bilingualism in English and an official language. Hence, the English signages in all the hospitals are the first insurmountable barriers for the LDOAs. Without guides, they are unable to negotiate the hospital labyrinth.

The pinnacle of this obstacle course is the language-discordant encounters with their doctors. All respondents pointed out the limitations of the 


\section{$156 \mathrm{Ng}$ Bee Chin and Francesco Cavallaro}

Chinese language competency of doctors, even of those who are ethnically Chinese. The following are some of the responses. (Note that the responses are translated from Chinese languages as none of the LDOAs were able to speak English.)

- "They [the doctors] do not speak Mandarin at all." (FM74)

- "Some Chinese doctors cannot speak Mandarin well ..." (FM78)

- "That day, I consulted a Cantonese doctor, and he was unable to speak Hokkien. He asked if I was able to speak Mandarin. I said if you speak in simple and basic terms, I will be able to understand. But if you get too complicated then I will not be able to understand." (MH81)

- "I can understand simple terms." (FH82)

Anything complicated posed a challenge for some of these LDOAs. With the limited Chinese-speaking competencies of the doctors, the level of understanding by the patients is consequently compromised, as seen in the responses below:

- "They [the doctors] speak in English; I do not understand." (FM77)

- "I am able to understand the Chinese doctors better, however sometimes they also cannot speak Mandarin well, and they have to ask the nurses to interpret." (FM78)

The LDOAs repeatedly emphasized their doctors' usage of English or limited use of Mandarin, and their consequent inability to understand or be engaged in a conversation without the aid of ad hoc interpreters. This is further illustrated on in the following section.

\subsubsection{Reliance on Ad Hoc Interpreters}

The responses of the elderly patients reveal a reliance on ad hoc interpreters, especially on their children and nurses. A common thread across responses given is that as doctors tend to be assisted by nurses, the LDOAs took for granted that the nurses' job scope includes being interpreters.

- "He [the doctor] will ask a nurse to come into the room ... the nurse who can speak Hokkien will interpret for me." (FH72)

- "When they [the doctors] speak in English I am unable to understand, unless there is an interpreter [a nurse] next to the doctor." (FM77)

- "If I don't understand, I look for nurses and ask them." (FM77)

- "If the doctors cannot speak Mandarin, they will ask others such as 'missy' (nurses), administrative staff, or people who do chores in the hospitals for help" (FM70)

The nurses functioned as mediators between patients and doctors and they were also the hospital personnel whom patients sought out when faced with 
difficulties in understanding or any other uncertainties. Some of the LDOAs saw resorting to nurses as the only option as seen below:

- "As we cannot speak in English, there is no choice but to ask someone in to help us understand better. If we meet a Hokkien nurse, we can at least tell her that things are like this and that and she will understand us." (FH72)

- "There's no choice even if it is troublesome. We have to ask around if we do not understand." (FM78)

With a quarter of doctors in Singapore being foreign and the high number of non-Singaporean nurses and other healthcare workers, as well as the number of younger Singaporeans who may not know local languages or even Mandarin, the nurses who are able to provide this service is decreasing. Indeed, some LDOAs reported that doctors have resorted to asking clerical or administrative staff in the office for assistance when no nurses with the language ability could be found. In the following excerpt, FM77 reported that a fellow patient in the ward pitched in as an ad hoc interpreter.

- “There was once my neighbour's (the patient in the bed next to her) visitor had to help to translate what the healthcare professionals were saying to me as Hospital A has many foreign doctors and nurses who can only speak English." (FM77)

\subsubsection{Family Members as Interpreters}

It was also common for these LDOAs to be accompanied by family members who would speak on their behalf during their healthcare visits.

- "My daughter will speak for me. There is no need for me to speak." (FC72)

- "My son and the doctor would speak in English, a language that I do not understand. I will then ask my son about what the doctor say, and he will explain to me." (FC72)

- "I use Hokkien and she (my daughter) will interpret it into English for the doctor." (FH72)

The responses above, once again underscore the notion of dependence - in this case, on family members. The following is an example of an LDOA who went for the first time to the hospital without her children as she was not anticipating any significant language problem. Her coping mechanism was to remain silent despite completely not understanding what her doctor was saying:

- "There was once I went for a hospital visit alone without any company, I did not understand anything and chose to stay silent and just leave it. 
I simply took my medicine, paid the bill, and went home. Hence, for subsequent visits, my children have to accompany me." (FM74)

In another case, despite having an underlying desire for autonomy, another LDOA allowed her son to speak on her behalf, because she felt that her son was more likely to take time to explain things to her, unlike a doctor. However, FH79 still clearly preferred hearing the information directly herself instead of from her son.

- "It will be much clearer when we hear it for ourselves. It may not be that clear if my son listens on my behalf. My son might forget some things that the doctor says. When we hear it ourselves, we will put in more effort to remember what is being said." (FH79)

Using family members as interpreters is low cost and convenient, but this strategy is not without its problems. This strategy deprives the LDOAs of a sense of autonomy and it also deprives them of the possibility to be their own health advocate. This disempowerment has other negative knockon effects. The case of FM74 who pretended to understand the consultation is potentially dangerous and posed a serious risk to her well-being. Medication compliance for older adults who have multiple chronic conditions can be very complicated and any error in following instructions due to language barriers have been found to have very disastrous ramifications (Suppiah et al. 2020).

\subsubsection{Frustration and Passive Acceptance}

Overall, the LDOA participants indicated that, despite the frustration of not having a common language, they still trusted and complied with the doctor's instructions. For example, MM68 felt that it was not important to understand everything his doctor said.

- "Just catching the approximate meaning is sufficient, I do not need to think so much about it." (MM68)

From a medical viewpoint, this passive acceptance of language barriers can be a problem. As pointed out earlier, the consequent inability of LDOAs to make informed health decisions can be detrimental if they have limited health literacy and are unable to sufficiently comprehend health information in language-discordant medical encounters (Capps, Rolfe, and Logsdon 2016).

Only one LDOA in our group was assertive enough to ensure she understood what the healthcare professionals were saying by insisting on repetitions and clarifications. Several on the other hand, expressed their frustration, especially when recounting their hospitalization experiences: 
- "Whenever an urgent need comes up and no one can communicate in Mandarin, it becomes frustrating for me as I have no choice but to use gestures." (MM68)

- "When they (the hospital) are shorthanded and I am really left without choice, especially during night shifts, I have to attempt to use broken Chinese or extremely basic English words to get my message across." (FM78)

The frustration and sense of desperation is evident in the above excerpts and such emotional distress and anxiety was common in the account of their healthcare encounters. The next sections provide an account of the views of some health professionals.

\subsection{Perspective From the Nurses}

The interviews were conducted in English with three nurses who worked in public hospitals and interacted with a significant number of LDOAs in the course of their careers. The first nurse interviewed, Eve (all names are pseudonyms), was 29 years old and had two years of nursing experience in public hospitals. She was fluent in English, Mandarin, and Cantonese, and she was able to speak basic Malay and Hokkien. The second nurse, Dorcas, was 71 years old and had 53 years of nursing experience in public hospitals and mobile clinics. She was fluent in English, Mandarin, Hokkien, Teochew, and Cantonese, and some basic Malay. The last nurse, Lucy, was 69 years old and had 50 years of experience as a nurse in public hospitals and polyclinics. She was fluent in English, Mandarin, Hokkien, and Cantonese, with basic Malay, Teochew, and Hakka. The recurring themes these nurses encounter at work in public hospitals are as follows:

- A focus on "getting by" medical encounters each day,

- Their own reliance on ad hoc interpreters and their role as ad hoc interpreters,

- Common concern of time wastage and distraction from core responsibilities.

\subsubsection{Focus on "Getting By" in Medical Encounters}

The responses of the nurses reveal that simply being able to "get by" is of primary concern to them during medical encounters. Tang et al. (2014) defined "getting by" as knowing that the practice of using an ad hoc interpreter is not ideal but doing it anyway as it was the only solution. "Getting by" also involves omitting information if no interpreters were available. Basically, "getting by" means you just make do with whatever resources you have. 


\section{$160 \mathrm{Ng}$ Bee Chin and Francesco Cavallaro}

- "If we [the nurses] cannot find a translator, we will call the patients' family members. If we cannot get through, then there is no choice (an attempt to communicate stops)." (Eve, 29)

- "Sometimes when we [the nurses] are left without choice because we really cannot find the people who can speak the required language, we have to make do with limited communication and understanding." (Lucy, 69)

\subsubsection{Reliance on Ad Hoc Interpreters}

It is clear from the three nurses that the use of $a d$ hoc interpreters was prevalent in medical encounters as gathered from the nurses' responses:

- "During interactions with my patients, I will ask my colleagues or the patients' children to translate for me if I do not know how to speak the language well enough." (Lucy, 69)

- "If I face difficulty communicating with patients, I rely on existing staff, the family members at their bedside or their next-door patients to translate for me." (Eve, 29)

Similar to the LDOAs in this study, these nurses also relied extensively on ad hoc interpreters, which consisted of their colleagues and the LDOA's children. When these groups of people were unavailable, the nurses sought help from other patients or other hospital staff, or anyone else who was happened to be there.

\subsubsection{Nurses' Role as Ad Hoc Interpreters}

As indicated earlier, LDOAs tended to see nurses as one of the main groups of ad hoc interpreters they can rely on. These nurses felt the pressure of this reliance on a day to day basis.

- "I can be called to be an interpreter a few times a day." (Dorcas, 71)

- "The frequency of me being an ad hoc interpreter is about 20 to $30 \%$." (Eve, 29)

Part of making do means that the nurses often had to interpret outside their comfort zone:

- "Sometimes when I communicate in languages that I am weak in, such as Malay, I will have trouble expressing myself or understanding the patients." (Lucy, 69)

- "For me, communicating is not much of an issue but sometimes it gets challenging when I have to speak in Malay." (Dorcas, 71) 
Their struggle with the responsibility of having to interpret in languages they were not so familiar with was a source of stress and anxiety. Dorcas expressed her guilt, worry, and struggle as she grappled with communicating in her less fluent languages. Eve acknowledged her limitations in medical encounters of greater complexity as she struggled to relay the message accurately, especially when they involved the use of medical terminologies:

- "Usually, I have no problem communicating the basic things in different languages even with my very limited Hokkien and Malay language skills. However, once the subject matter gets more complicated, for example, when I have to use medical terms in a patients' language, I face problems going in-depth even if I am proficient in the language. This can get very stressful" (Eve, 29)

\subsubsection{Concerns Over Time Wastage and Distraction From Core Responsibilities}

The element of time lost or wasted was one of the foremost concerns for nurses as they repeatedly raised time wastage as a negative yet inevitable implication of language-discordant medical encounters:

- "If I cannot communicate with the patients, I may use gestures. However, it takes a longer time." (Lucy, 69)

- "At times, there will be a lot of impatience, frustration, and unhappiness from both patients and doctors when they have to search and wait for an ad hoc interpreter" (Dorcas, 71)

- "If I am doing something, I have to ask them (people who need ad hoc interpreters) to wait." (Lucy, 69)

They were aware of how efforts to mitigate or circumvent language barriers such as the use of gestures, or the searching and waiting for the availability of ad hoc interpreters often resulted in more time wasted which consequentially created frustration and unhappiness in both patients and healthcare professionals. A common thread in their responses was the negative distraction that ad hoc interpretation brought to their work:

- "It takes time from my own ward." (Lucy, 69)

- "When you come back to your own room (ward) after interpreting for others, it is in a mess. There is no choice but to neglect my own room when I am called to be an interpreter. There is also no one to help to take over my room as everyone else is too busy." (Dorcas, 71)

- "Being an ad hoc interpreter causes me to neglect my own patients." (Eve, 29) 


\section{$162 \mathrm{Ng}$ Bee Chin and Francesco Cavallaro}

They all saw this distraction as compromising of the quality of care received by all patients - not only the LDOAs needing language assistance.

\subsection{Perspective From the Doctors}

The two doctors interviewed worked in public hospitals and have encountered a significant number of LDOAs in their professional lives. Both of the doctors interviewed were Chinese Singaporean females (29 years old) and both had four years of experience working in public hospitals. The first doctor, Faith, was fluent in English and Mandarin, and was able to converse in minimal Malay and Hokkien. The second doctor, Rachel, was likewise fluent in English and Mandarin, with minimal knowledge in Malay and a variety of dialects. Two recurring themes have been identified from the responses of these doctors:

- A similar focus on "getting by."

- An acceptance of the "getting by" philosophy.

\subsubsection{Focus on "Getting By" in Medical Encounters}

Like the nurses, the doctors' focus on "getting by" is clear.

- "Professional interpreters are only used in language encounters which involve rare languages like Arabic or Japanese. For the usual national languages, I will just ask for help from existing staff." (Rachel, 29)

- "I will get patients' family members or healthcare workers to help with translation when I am faced with language barriers in communication." (Faith, 29)

The responses confirmed that the use of ad hoc interpreters, most commonly healthcare staff and LDOAs' family members, was one of the primary ways through which doctors coped with language barriers. Although, there was an awareness that this reliance on ad hoc interpreters had its limitations:

- "It is hard to tell how accurate ad hoc interpreters are, as I do not understand the language myself. I would assume that if the interpreters are nurses or other healthcare professionals, interpretation would be better and more accurate, whereas if I ask the administrative staff for help, I think that they also struggle with interpretation." (Faith, 29)

Faith admitted that she was unable to ascertain the effectiveness or accuracy of ad hoc interpretations. The fact that ad hoc interpreters are ultimately untrained, coupled with a doctors' inability to gauge the effectiveness or outcome of communication, opens the possibility for undetected erroneous 
or incomplete interpretation. Other examples of this "getting by" strategy are shown in the following excerpts.

- "I can understand and speak a little dialect [Chinese vernaculars], so I can still get by in these encounters." (Faith, 29)

- "Occasionally, when I face difficulty in expressing myself, I will add in some broken English to try to get my message across." (Rachel, 29)

Faith acknowledged the difficulty when there was a need to use medical terminologies or when she had to talk about more complicated issues. The doctors' response below highlights the potential problem of ineffective communication posed by the "getting by" approach:

- "Whenever I try to explain things in Mandarin or dialect, especially when it comes to medical terminologies or more complicated issues, language barrier becomes significant." (Faith, 29)

- "Many of my colleagues and I cannot speak Mandarin well, so in communicating with patients some meaning is inevitably lost along the way. When it comes to medical terms, this [loss] becomes quite significant." (Rachel, 29)

\subsubsection{An Acceptance of the "Getting By" Philosophy}

Though the doctors were cognizant of the problems and potential dangers of this "getting by" approach, there is a sense that they perceived this to be an accepted norm and hence, not something that is likely to change.

- "[In utilizing ad hoc interpreters] We have been able to get by with no serious mistakes; professional interpreters are not crucial, but will be belpful." (Rachel, 29)

- "Language barrier compromises the quality of care received by patients to a little extent as it does not really affect their health or recovery; it is just that these patients may not understand as much as they could have, but it is not anything dangerous or unsafe." (Rachel, 29)

Basically, though the circumstances deviated from the ideal treatment, they were considered acceptable and, according to the doctors, did not compromise care in any way.

It is interesting that both doctors' trust in the safe functioning of the "getting by" approach is not in tune with the research on the importance of sound communication and patient care. In a review of 36 papers on this topic, Flores (2005) presents a correlation between the lack of professional interpretation with poorer patient satisfaction, quality of care, and health outcomes. This is not only measured in terms of better overall health outcomes for the patients, but also reflected in a significant reduction of cost 


\section{$164 \mathrm{Ng}$ Bee Chin and Francesco Cavallaro}

to the healthcare system. Jacobs et al. (2004) report that when patients are provided with professional interpretation services, they are more likely to make proactive health choices resulting in healthcare savings, which are nine times the cost of providing a professional interpreter. A more recent review by Brandl, Schreiter, and Schouler-Ocak (2020) continues to support the call for more widespread use of professional interpreters, indicating that it benefits both the patients and the healthcare systems. The sense of complacency shown by the doctors in our study may in itself indicate a dangerous complacency which should be a public health concern. In short, "getting by" is just not "getting it."

\section{Discussion}

From the responses of all three groups of respondents it is clear that the patients and health professionals experience language-discordant encounters and often had to face the repercussions of this problem. These challenges faced, and the consequent compromise in their communications, inevitably led to frustrations experienced by the LDOAs. Similarly, nurses - who were often called away from their core duties to be an ad hoc interpreters - also recognized the problems in such encounters in terms of poor communication, inefficiency, and compromised patient care.

The doctors, on the other hand, have accepted the "getting by" philosophy all too readily. This has culminated in a mindset that sees the use of ad hoc interpreters as a benign phenomenon and, therefore, the status quo. While it is true that there have been no overt disasters that can be attributed to the use of $a d$ hoc interpreters, there are hidden costs to the health system, such as patients' infrequent visits to healthcare providers, delayed treatments, clinical errors, an increased susceptibility to chronic diseases, and health complications (Capps, Rolfe, and Logsdon 2016; Coren, Filipetto, and Weiss 2009).

To summarize, there are three key problems associated with the current practice. First, these ad hoc interpreters may possess a varying degree of proficiency in the vernacular or Mandarin when attempting to interpret. Second, leaning on nurses as a source for interpretation can overburden the nurse, and this interruption of their core duties has been found to be a stressor that distracts nurses and interferes with their workflow. This has other implications as well, as Duchêne puts it $(2011,102)$, the language skills of these semi-visible workers "are not considered worthy of recognition ... they are inventoried and can be called upon - in this sense, they are exploitable - when the institution needs them to successfully complete its work." He goes on to argue that the company itself, in this case the hospital, is the only beneficiary of this mode of communication. Third, the interpretation done by ad hoc interpreters may not be fully representative of the LDOA patients' actual health condition, especially as they are not trained 
medical interpreters, and they may not know the correct medical terms. This results in situations where medical information may be lost in the process of relaying the doctor's message.

\section{Conclusion}

This chapter provides an account of the language barriers experienced by LDOAs in medical encounters in Singapore. The inadequate existing measures exposes our older adults to potential risks of clinical errors and of subjecting them to a compromised quality of care. The medical encounters are emblematic of the other daily linguistic challenges they have to face. On an individual level, this language displacement has resulted in the disruption in intergenerational communication due to differing language repertoires between the younger and older generations and contributes to a keen sense of isolation felt by these older adults. Aging on its own is not without its challenges, but for this group of older adults who are displaced, the challenges are multi-fold. They have been deprived of their familiar sights and sounds, but as a society, at least in the very important healthcare settings, we could provide them with the means for them to feel cherished and empowered. Such means are readily available in the community in the form of well-trained multilingual facilitators. From the point of view of linguistic justice, these LDOAs have been marginalized by a system that privileges one language over another. In the schema of linguistic justice articulated by (Van Parijs 2002, 2011), the evaluation of linguistic justice is always synchronic and does not take into account historical change and evolution and the casualties in the process. Cross-border English hegemony and its initial inequality is seen as both inevitable and unavoidable. In the case of the LDOAs in Singapore, the diachronic within-border experience of the different forms of multilingualism is certainly a key factor in their experience of linguistic anomie. Younger generations born into the Singaporean Englishdominant economy may have bought into it and, therefore, minimized the effect of the linguistic injustice on them. However, for this aging group of LDOAs, the spread of English within their community has definitely given rise to unequal multilingualism within their national borders and within their lifetime. We believe that the invisible voices of these LDOAs are precisely what Lanza and Lomeu Gomes (2020) identified as one of the multilingual practices that are not addressed in the Global North, which so far has been very engaged in migration and transnationalism studies. Singapore joins the rank of 33 other nations to be "superaged" in ten years where $22 \%$ of the population will be over 65 . This number is set to increase to $34 \%$ in 2050. Hopefully, it will have a multilingual workforce to adequately cater to this burgeoning population as well as that of the more than 1 million migrant workers. 


\section{$166 \mathrm{Ng}$ Bee Chin and Francesco Cavallaro}

\section{References}

Arthur, K., R. Mangione-Smith, H. Meischke, C. Zhou, B. Strelitz, M. Garcia, and J. Brown. 2015. "Impact of English Proficiency on Care Experiences in a Pediatric Emergency Department." Academic Pediatrics 15 (2): 218-224.

Attard, M., A. McArthur, D. Riitano, E. Aromataris, C. Bollen, and A. Pearson. 2013. "Improving Communication between Health-care Professionals and Patients with Limited English Proficiency in the General Practice Setting." Australian Journal of Primary Health 21 (1): 96-101.

Betancourt, J.R., M.R. Renfrew, A.R. Green, L. Lopez, and M. Wasserman. 2012. Improving Patient Safety Systems for Patients with Limited English. Agency for Healthcare Research and Quality. Cambridge, MA: Agency for Healthcare Research and Quality.

Brandl, E.J., S. Schreiter, and M. Schouler-Ocak. 2020. “Are Trained Medical Interpreters Worth the Cost? A Review of the Current Literature on Cost and Cost-Effectiveness." Journal of Immigrant and Minority Health 22 (1): 175-181.

Capps, J., S. Rolfe, and C. Logsdon. 2016. "Limited English Proficiency: Impact on Health Literacy and Health Disparity." The Kentucky Nurse 64 (1): 13-14.

Cavallaro, F. and B.C. Ng. 2014. "Language in Singapore: From Multilingualism to English Plus." In Challenging the Monolingual Mindset, edited by Y. Slaughter and J. Hajek, 33-48. Bristol, UK: Multilingual Matters.

Cavallaro, F. and B.C. Ng. 2020. "Chapter 8: Multilingualism and Multiculturalism in Singapore." In Multilingual Global Cities: Singapore, Hong Kong and Dubai, edited by P. Siemund and J.R.E. Leimgruber. New York, USA: Routledge.

Cavallaro, F., B.C. Ng, and Y.-Y. Tan. 2020. "Singapore English.” In Handbook of Asian Englishes, edited by Kingsley Bolton, Weerner Botha, and Andy Kirkpatrick, 419-448. New Jersey, USA: Blackwell-Wiley.

Cavallaro, F., M.F. Seilhamer, Y.T.F. Chee, and B.C. Ng. 2016. "Overaccommodation in a Singapore Eldercare Facility." Journal of Multilingual and Multicultural Development 37 (8): 817-831.

Coren, J., F. Filipetto, and L. Weiss. 2009. "Eliminating Barriers for Patients with Limited English Proficiency." The Journal of The American Osteopathic Association 109 (12): 634-640.

Department of Statistics. 2019. Population Trends 2019. Singapore: Department of Statistics, Ministry of Trade \& Industry.

Duchêne, A. 2011. "Neoliberalism, Social Inequalities, and Multilingualism: The Exploitation of Linguistic Resources and Speakers." Langage et société 136 (2): 81-108. doi: 10.3917/ls.136.0081.

Flores, G. 2005. "The Impact of Medical Interpreter Services on the Quality of Health Care: A Systematic Review." Medical Care Research and Review 62 (3): 255-299.

Gan, K.Y. 2012. "Speech by Mr Gan Kim Yong, Minister for Health at The Groundbreaking of the Lee Kong Chian School of Medicine's Novena Campus." https://www.asiaone.com/health/coming-more-doctors-2020.

Jacobs, E.A., D.S. Shepard, J.A. Suaya, and E.-L. Stone. 2004. "Overcoming Language Barriers in Health Care: Costs and Benefits of Interpreter Services." American Journal of Public Health 94 (5): 866-869.

Karliner, L., E. Jacobs, A. Chen, and S. Mutha. 2007. "Do Professional Interpreters Improve Clinical Care for Patients with Limited English Proficiency? A Systematic Review of the Literature." Health Services Research 42 (2): 727-754. 
Lanza, E., and R. Lomeu Gomes. 2020. "Family Language Policy: Foundations, Theoretical Perspectives and Critical Approaches." In Handbook of Home Language Maintenance and Development: Social and Affective Factors, edited by A.C. Schalley and S.A. Eisenchlas, 153-173. Berlin: De Gruyter Mouton.

Lanza, E. and H. Woldemariam. 2014. "Indexing Modernity: English and Branding in the Linguistic Landscape of Addis Ababa." International Journal of Bilingualism 18 (5): 491-546.

Mokhtar, Maryam. 2014. "Parliament: More Than 2,000 Foreign Nurses Went for Language Courses in last 5 Years.” The Straits Times. https://www.asiaone. $\mathrm{com} /$ health/parliament-more-2000-foreign-nurses-went-language-courses-last-5years.

Meyer, B., B. Pawlack, and O. Kliche. 2010. "Family Interpreters in Hospitals: Good Reasons for Bad Practices.” mediAzioni 10 (Special Issue 2010): 297-342.

Neo, C.C. 2010. "Communicating with Patients: How Do You Say 'Healthcare' in Hokkien? Today Newspaper Singapore.” Today. https://eresources.nlb.gov.sg/ newspapers/digitised/issue/today20100301-3.

Ng, B.C., and F. Cavallaro. 2021. "The Case of Mandarin Chinese in Singapore.” In Multilingual Singapore: Legacy, Status, and Prospects, edited by Ritu Jain. New York, USA: Routledge.

Ngo-Metzger, Q., D. Sorkin, R. Phillips, S. Greenfield, M. Massagli, B. Clarridge, and S. Kaplan. 2007. "Providing High-Quality Care for Limited English Proficient Patients: The Importance of Language Concordance and Interpreter Use.” Journal of General Internal Medicine 22 (2 Supplement): 324-330.

Regan Freeman, R. 2014. "Primary Care for Limited English-speaking Patients and Parents." Journal of The American Association of Nurse Practitioners 27 (3): 167-176.

Rosenberg, E., R. Seller, and Y. Leanza. 2008. “Through Interpreters' Eyes: Comparing Roles of Professional and Family Interpreters.” Patient Education and Counseling 70 (1): 87-93.

Schenker, Y., E. Pérez-Stable, D. Nickleach, and L. Karliner. 2011. "Patterns of Interpreter Use for Hospitalized Patients with Limited English Proficiency." Journal of General Internal Medicine 26 (7): 712-717.

Schyve, P. 2007. "Language Differences as a Barrier to Quality and Safety in Health Care: The Joint Commission Perspective.” Journal of General Internal Medicine 22 (2 Supplement): 360-361.

Suppiah, S., Y.W. Tan, G.H.-L. Cheng, W.E. Tang, and R. Malhotra. 2020. "Mediators of the Association of Limited English Health Literacy with Medication Non-adherence among Singaporean Elderly." Proceedings of Singapore Healthcare 29 (1): 25-32. doi:10.1177/2010105819899126.

Tan, S. and B.C. Ng. 2010. "Three Generations Under One Roof: A Study of the Influence of the Presence of Grandparents on Language Shift, Identity and Attitudes." TRANEL Travaux neuchâtelois de linguistique 52: 69-92.

Tang, A., J. Kruger, J. Quan, and A. Fernandez. 2014. "From Admission to Discharge: Patterns of Interpreter Use among Resident Physicians Caring for Hospitalized Patients with Limited English Proficiency." Journal of Health Care for the Poor and Underserved 25 (4): 1784-1798.

TST. 2015. "Number of Foreign Doctors Rising in Singapore Public Hospitals and Polyclinics." The Straits Times. http://www.straitstimes.com/singapore/health/ number-of-foreign-doctors-rising-in-singapore-public-hospitals-and-polyclinics. 


\section{$168 \mathrm{Ng}$ Bee Chin and Francesco Cavallaro}

Van Parijs, P. 2002. "Linguistic Justice.” Politics, Philosophy \& Economics 1 (1): 59-74.

Van Parijs, P. 2011. Linguistic Justice for Europe and for the World. Oxford: Oxford University Press.

Williams, S., J. Weinman, and J. Dale. 1998. "Doctor-patient Communication and Patient Satisfaction: A Review.” Family Practice 15 (5): 480-492. 


\title{
8 Fostering Storytelling by Persons with Dementia in Multiparty Conversation
}

\author{
Jan Svennevig and Heidi E. Hamilton
}

\section{Introduction}

Telling personal stories is important for persons with dementia (PWDs), both for maintaining a sense of personal identity (Ramanathan 1995; Shenk 2005; Hamilton 2008; Hydén and Örulv 2009; Hydén 2018; Hamilton 2019) and for establishing or affirming social relations with others, such as staff and residents in care facilities (Crichton and Koch 2011) and spouses, family, and friends (Hydén 2011, 2018). However, PWDs frequently find that communicative and cognitive problems (such as finding words or recalling past events) may get in the way of participating in conversation on a par with their healthy conversation partners (Hamilton 2019; Wray 2020). As a consequence, PWDs may become relatively passive in their communication with others, taking fewer initiatives in conversation, limiting themselves instead to responses to others' questions of them (Backhaus 2018). Multiparty interactions can present special challenges, as the pace of the conversation and its more complex participation framework may make it difficult for PWDs to get the floor and hold it. A further complication may be involved if the PWD is to tell the story in a second language.

In order to counteract such problems, spouses, friends, and personal and professional carers may seek to assist PWDs in getting the floor and telling a story. Through conversational practices facilitating distributed cognition and distributed agency (Schrauf and Müller 2014; Hamilton 2019; Landmark and Svennevig, forthcoming), PWDs may find themselves in a position to make complex conversational contributions and share detailed personal experiences. Interlocutors may encourage PWDs to speak by asking questions and providing contextual clues (Williams et al. 2019) and engage in narrative scaffolding by completing and repairing utterances produced by the PWD (Hydén 2011). Even if they take on the role as primary storyteller, interlocutors may use verbal and non-verbal practices to involve the PWD in the telling and thereby construct the story as a collaborative achievement (Nilsson, Ekström, and Majlesi 2018).

However, such practices may on occasion be detrimental to the PWDs' participation and agency. Questions and prompts to tell may develop into 
test-like situations, causing problems and embarrassment for PWDs if they do not remember the requested information (Nilsson 2017). In addition, interlocutors who are privy to the details underlying a particular story may end up taking over the floor and speaking for PWDs instead of allowing them to speak for themselves (Österholm and Samuelsson 2015). The interactional challenge, then, is this: what interactional practices can conversational partners implement to assist PWDs in putting their remaining communicative resources to best use in storytelling? The current study investigates storytelling by PWDs in two extended excerpts from multiparty conversation, one from a domestic context, a sociable lunchtime conversation with friends, and one from an institutional context, a weekly meeting of an early-memory-loss support group. It focuses on the interlocutors' interactional practices that prove successful in promoting and scaffolding the PWDs' storytelling without putting them on the spot or speaking for them.

\section{Collaborative Storytelling in Conversation}

Storytelling in natural conversation is in essence a collaborative achievement and is in fundamental ways shaped by the actions of all interlocutors. Recipients are central in establishing the relevance of telling a story at a certain point in a conversation. Story prefaces generally include a slot for the recipient to accept or block the proposed storytelling (Sacks 1974). If recipients give a green light, they signal their willingness to allow the storyteller a multi-unit turn, and subsequently show their alignment as story recipients by restricting themselves to certain forms of minimal response, such as continuers, nods, and assessments (Stivers 2008). Other responses, such as clarification questions and other forms of "byplay" are generally produced in ways that minimize their intrusiveness to the progression of the story (Goodwin 1997). In cases where such byplay is prolonged, it may have serious disruptive consequences and threaten to derail the storytelling (Mandelbaum 1989). Finally, recipients are crucially involved in concluding the storytelling by displaying their appreciation of the story, thereby confirming the "tellability" of the story and its relevance to the topic of the conversation.

A special form of collaboration and coordination occurs in the presence of other participants who have epistemic access to the events reported, socalled "story consociates." As described by Lerner (1992), such participants may initiate storytelling by producing a story prompt and thereby eliciting a narrative from an interlocutor. In the body of the story, they may collaborate and take part in the storytelling by repairing potentially problematic aspects of the story or the storytelling, or by contributing parts of the story that crucially involve them as story characters, thereby taking the floor to "speak for themselves." And in the completion phase, they frequently add to the evaluation of the story by contributing their own assessment of the events reported in the story climax. 


\section{Data and Method}

The study comprises two instances of storytelling by a PWD. The first ("Passing the dinner rolls") comes from a casual conversation that occurred during a lunch in the home of the PWD ("Laura") and her husband with two invited friends. The conversation took place in Norway, but Laura, her husband and one of the friends were originally from the United States. Laura had previously been a native-like speaker of Norwegian, but at this point in time her Norwegian skills were markedly inferior to her English proficiency. The participants were all bilingual in Norwegian and English, and occasionally switched between these languages. The conversation was video-recorded by the husband according to the instructions of the researchers in the project "Language and Communication In Multilingual Speakers with Dementia" (for more details, see Svennevig et al. 2019).

The second case ("We used to be the caregivers") explores the trajectory of a story that emerged within the final few minutes of an audio-recorded weekly early-memory-loss support-group meeting. The one-hour interaction took place in an urban recreational center in the northeastern United States (for more details, see Hamilton and Baffy 2014) and included seven group members with dementia, two professionally trained group facilitators, and one visiting expert in dementia care.

The cases differ in two significant ways: 1) the type of encounter (everyday vs. institutional); and 2) the interlocutors' epistemic status (Heritage 2012) vis-à-vis the story to be told. In the casual conversation, the turn-taking and topic management were locally managed by the participants themselves. By contrast, in the institutional encounter, the professionally trained group facilitators usually held the reins of the discursive agendas: they chose topics for discussion, worked to ensure that the group stayed focused on the tasks at hand, and employed a range of discursive practices that encouraged and supported members' verbal participation. The cases also vary with regard to the epistemic status of the participants. In case 1, the story was prompted by the husband in a way that shows that he had previous knowledge of the events reported. In case 2, the story ultimately told by the PWD was not known by anyone else in the support group.

The study applies tools from Conversation Analysis (Sidnell and Stivers 2013) and Interactional Sociolinguistics (Gumperz 2015) to address realworld problems and identify possible solutions to them. By close analysis of single excerpts of naturally occurring interactions, we seek to understand the conversational mechanisms that promote or impede PWDs' storytelling in multiparty conversations and institutional talk. We use a multiple case study approach where we have purposefully selected two instances of storytelling in two very different interactions that, in our view, illustrate elements of good practice. They are "success stories" in two senses: 1) both PWDs recount aspects of relevant personal experiences to their conversational partners, thereby contributing positively to the development of the larger 
interaction; and 2) the interlocutors make use of conversational practices that effectively support the telling of these experiences.

In this way, we understand our study to be motivated by the "personhood movement" in dementia studies (cf. Kitwood 1997) in which "personhood" refers to "the person within - the reflexive, immaterial, communicable essence of a person that is located deep within the body, but that is sometimes veiled by symptoms" (Leibing 2006, 243). This move toward a focus on personhood is typically accompanied by a heightened interest in applying the findings of basic research to help PWDs and those who care for them, for example, by enhancing communication and lifting self-esteem. We align ourselves with most scholars in this area by focusing on the identification of active coping strategies and the enhancement of the social environment for the PWD.

\section{Passing the Dinner Rolls: Previously Known Story Told Within an Informal Setting}

The first case comes from a lunch conversation in the home of the PWD Laura (L) and her husband Gary (G), with their friends Anne (A) and Berta (B). Prior to the first extract, Anne excuses herself for stretching over to reach a plate of cheese, and Gary replies that the table is one that "requires long arms." Anne confirms by rephrasing this as "pension arms" - a Norwegian expression used to refer to stretching across the table when reaching for food. At that point, Gary turns to Laura and produces an explicit story prompt. As will become clear later on, the story relates to the problem of serving oneself when sitting at a large table: ${ }^{1}$

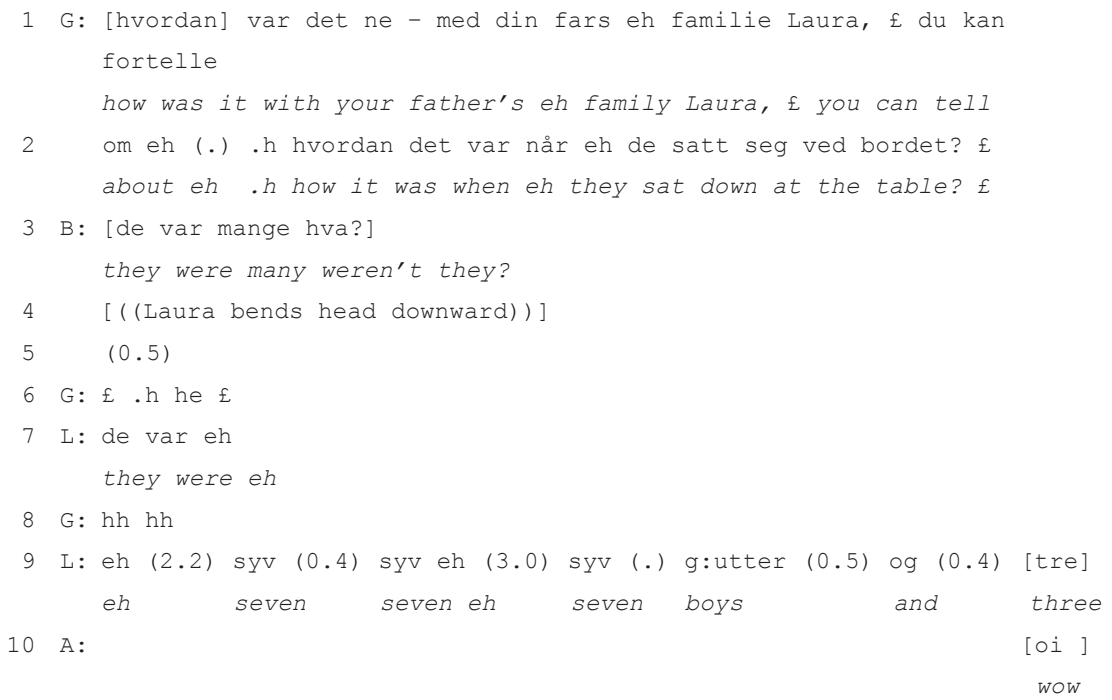




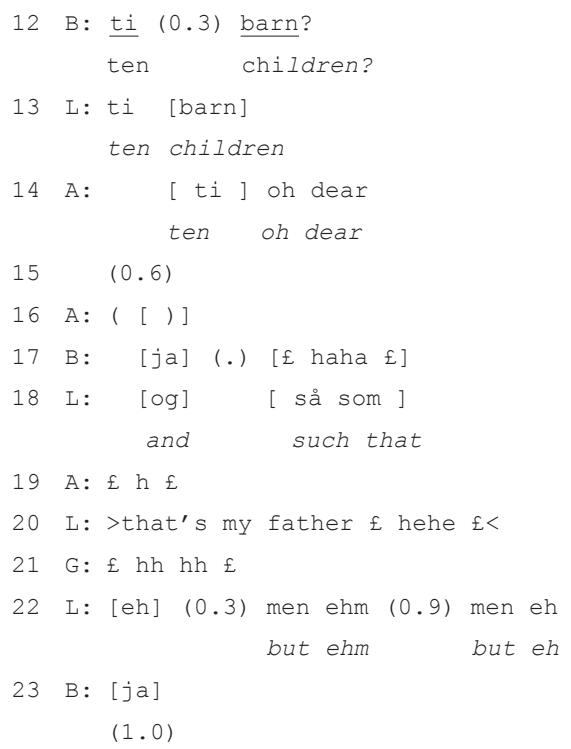

Gary's story prompt involves a reference to sitting down at a table, thereby displaying that the projected story is relevant to the topic of the prior talk and occasioned by it. This story preface shows that he is a "story consociate" but he orients to Laura's epistemic rights to tell the story by placing it within her primary epistemic domain (Raymond and Heritage 2006). $\mathrm{He}$ also gives her and the other participants a cue to identifying the type of story being projected by using a smiley voice and continuing to laugh in two subsequent pauses (lines 6 and 8). At the end of his utterance, Laura displays recognition by bending her head downward as if bursting into laughter (line 4). By producing this story prompt, Gary takes the role of a "broker," identifying the opportunity for Laura to make a relevant contribution to the conversation and inviting her to do so without speaking on her behalf.

At this point, the projected next action is for Laura to tell the story. However, simultaneously, Berta asks a clarification question eliciting background information relevant for understanding the projected story, thus initiating an insertion sequence (Schegloff 2007). As noted above, the preference for progressivity implies that such "byplay" is minimized in the service of returning to the main sequence and advancing the original project. However, Laura's answer generates expressions of astonishment (lines 10-14) and laughter (line 17) by the friends, which in turn leads Laura to expand the sequence further by producing a humorous comment about her father, code-switching to English (line 20). ${ }^{2}$

In line 22, Laura she seems to be marking a return from the insertion sequence in that she recycles the contrastive discourse marker "men" (but), which is commonly used as a resumption marker after temporary excursions 
from the main sequence (Mazeland and Huiskes 2001). However, the recycling and the subsequent pause (line 23) display trouble in resuming the main sequence. And at this point, Anne produces a question related to Laura's family:

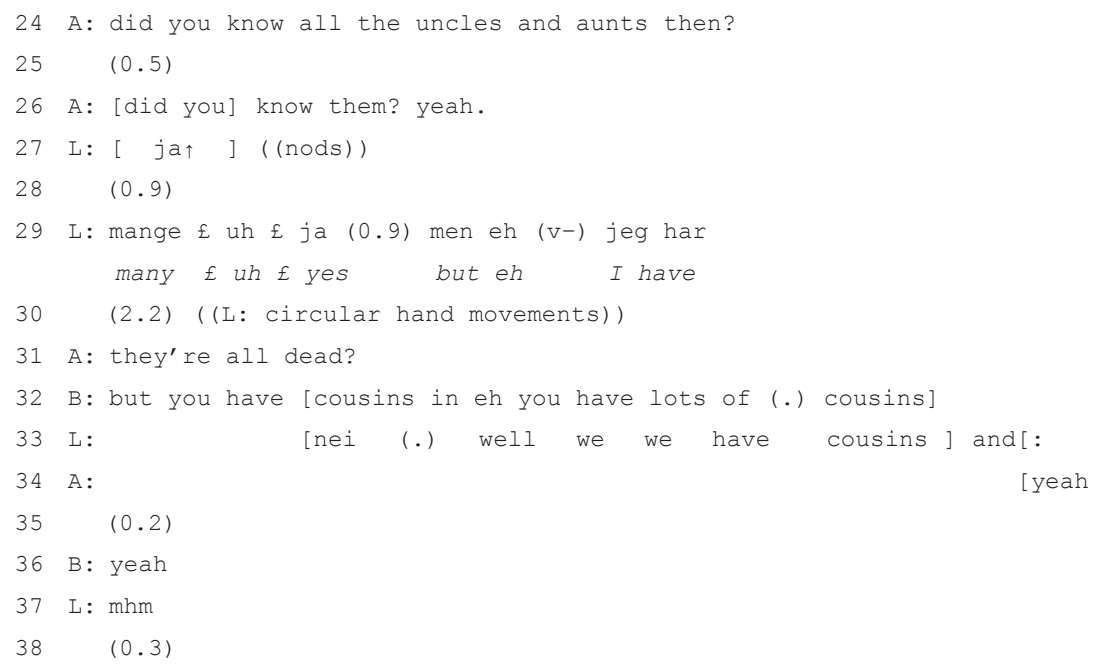

As we can see, the friends ask a series of questions about Laura's relatives, continuing in English. Laura answers the questions one by one. By continuing to ask questions related to the topic of the insertion sequence, the friends contribute to expanding it at the expense of letting Laura resume the storytelling activity. Questions like these may well be "well-intended," that is, oriented towards inviting participation by Laura, but in practice they seem to derail the storytelling. Toward the end of the excerpt, Laura does not try to resume the storytelling activity in the pauses that ensue (lines 35 and 38), and thus seems to have lost track of the story. At this point, Gary repeats his story prompt, and marks the resumption of the original activity by codeswitching back to Norwegian.

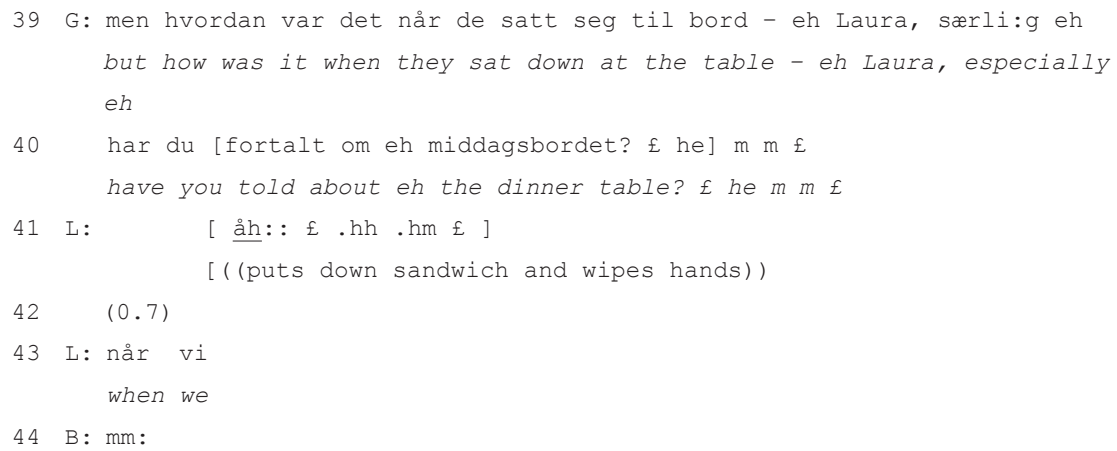




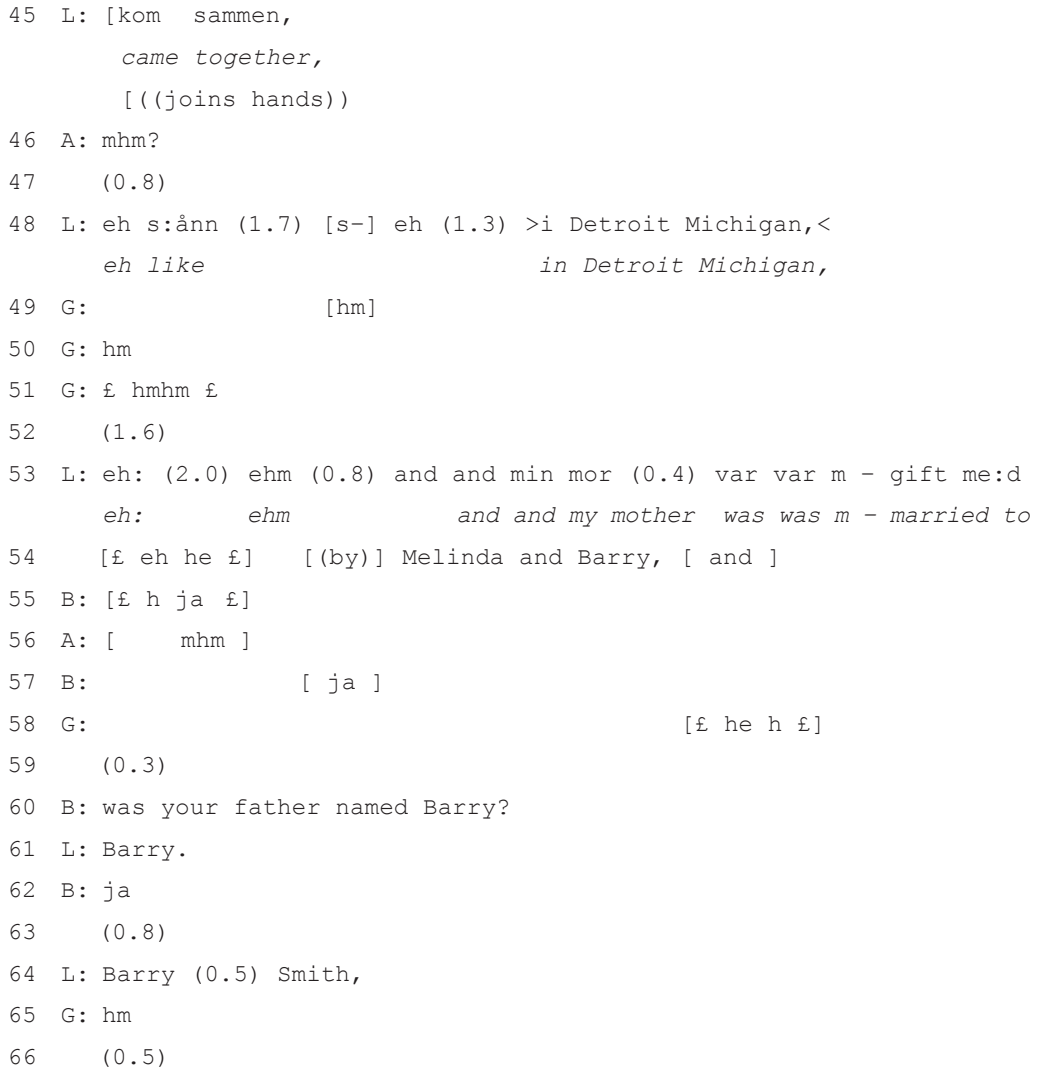

Gary's renewed story prompt effectively brings Laura back on track. This time, she makes a stronger display of recognition by producing a prolonged change-of-state token (“åh" - "oh”), indicating "now-remembering” (Seuren, Huiskes, and Koole 2016), followed by laughter tokens (line 41). She also displays readiness to start telling by putting down her sandwich and wiping her hands against each other, as if preparing for a new activity. She then starts producing what is hearably the prompted story, as it starts with the temporal subjunction "when," linking back to Gary's question about how it was "when they sat down at the table." ${ }^{3}$ This time, both Anne and Berta align as story recipients by producing continuers and letting her speak without interruption, despite her rather slow speech rate and the prolonged pauses. Gary aligns in a somewhat different way, producing short spurts of laughter (lines 49-51 and 58). As noted by Lerner (1992), story consociates may produce anticipatory laughter in order to project for the recipients a "laughable" in the upcoming talk. This seems particularly appropriate here since Laura at these points seems to deviate from the story by providing seemingly irrelevant details about the place and the participants (the story is about her father's childhood, and thus from a time long before he 


\section{6}

married). Gary's anticipatory laughter may thus not only be foreshadowing the humorous point to the recipients, but also to Laura, in order to assist her in staying on track with the storytelling. Laura again switches back to English in line 54 and this remains the language for the rest of the story. As in the previous extract, one of the friends poses a question (an understanding check) that contributes to topicalizing the information provided in this digression (line 60). In this way, Laura is once more distracted from the storytelling activity. However, as can be seen in the next excerpt, this time she manages to close the side sequence and return to the main sequence on her own:

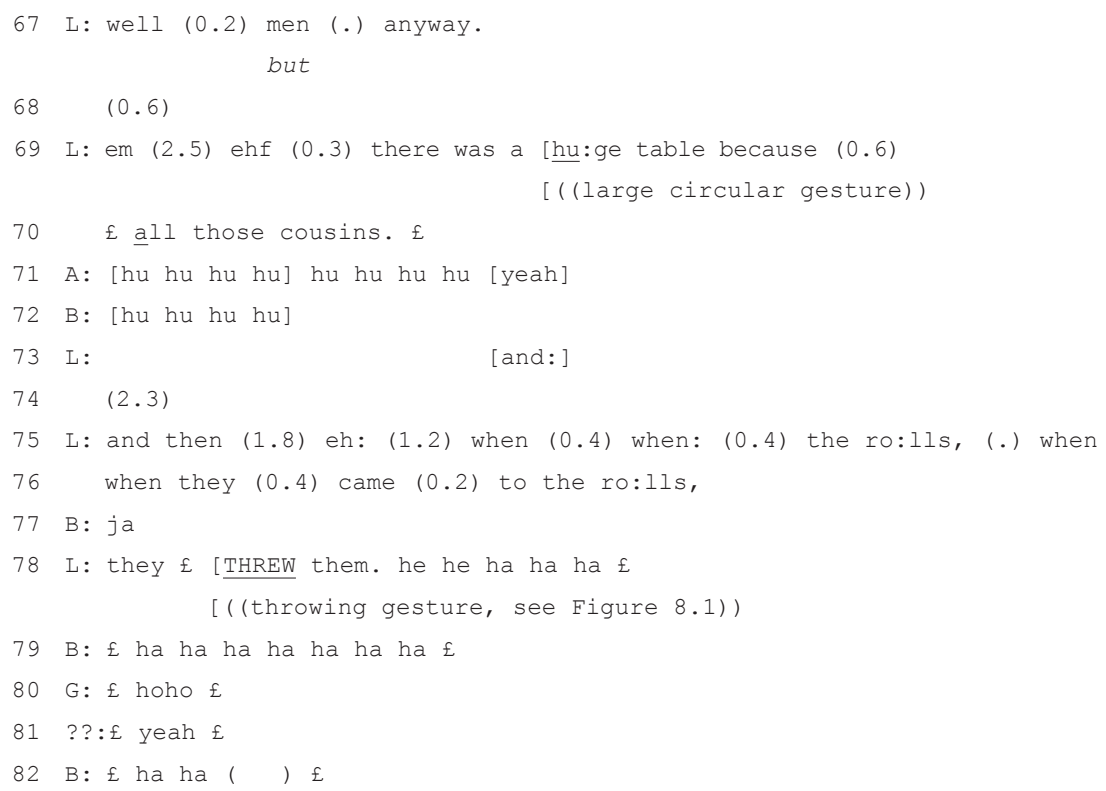

At the start of the excerpt, she produces several resumption markers, first in Norwegian ("men") and then in English ("anyway"), both signaling a return from the temporary excursion from the storytelling (Ottesjö 2005). And this time, she manages to keep the floor and advance the story, despite apparent production problems, leading to several prolonged pauses and self-repairs. At one point, she produces an utterance ("all those cousins") with laughter voice while leaning forward and gazing at Berta (line 70). Such displays of affective stance are commonly used in story climaxes (Selting 2017), and given that the projected point was a humorous one, Anne and Berta seem to treat this utterance as a potential punch line. They start to laugh, but Gary notably withholds any vocal response and thereby contributes to rejecting this interpretation of the turn. Laura then resumes the storyline by inserting the conjunction "and" (line 73), which gives her back control over the floor and projects continuation of the story. In the following extended pause 


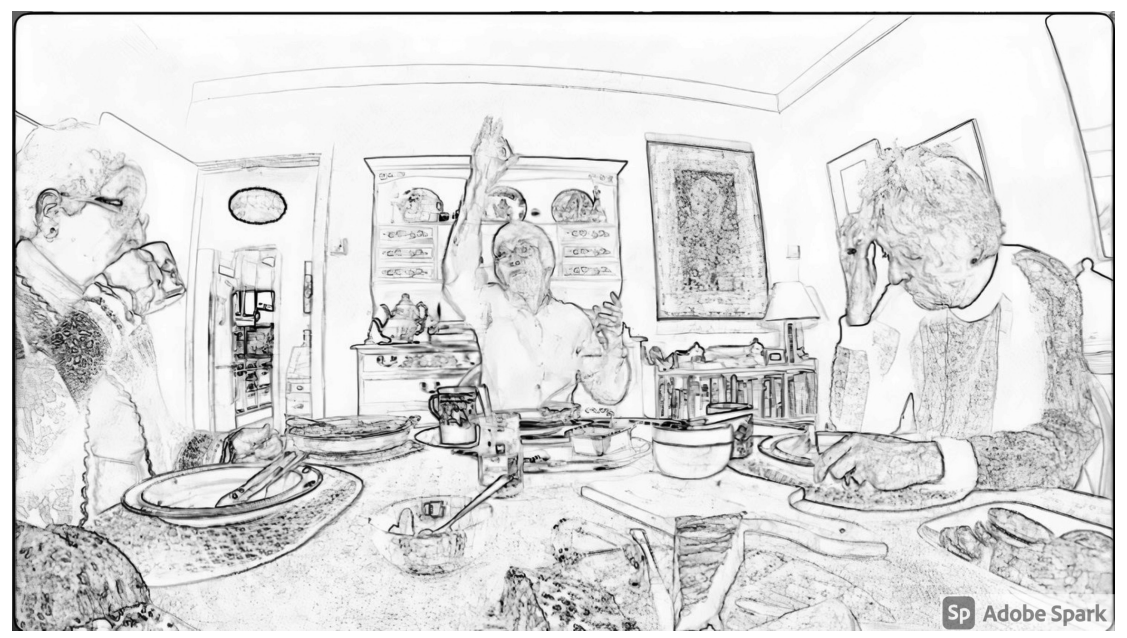

Figure 8.1 Throwing gesture

(line 74), the friends orient to this by withholding any talk. When Laura now comes to the punch line of the story, she marks heightened involvement by using laughter voice, high volume, and a large throwing gesture (Figure 8.1). This is an even more marked affect display and this time both the friends and Gary respond with extended laughter. The story may thus be considered successful in that Laura manages to deliver on her own the humorous point projected in Gary's story prompt.

What we can observe in this extract is thus a PWD who manages to contribute to a multiparty conversation with a humorous story that generates laughter. In doing so, she is prompted and helped by her interlocutors, but also distracted and derailed. The practices that promote the storytelling are primarily the husband's prompts. By asking her to tell a specific story, he both prompts her memory and gives her the floor in order to tell it. He thus does not speak on her behalf, even when she is derailed into talking about seemingly irrelevant matters. He consequently orients to her epistemic primacy and encourages her independent participation in the conversation. Furthermore, he also gives her time to talk, despite the slow pace and the many pauses, hesitations, self-repairs, and digressions that threaten the progressivity of the story and constitute points where interlocutors would typically enter into the speaker's turn space with collaborative completions or competing contributions. His continuers and laugh particles, produced as she speaks, align him as a story consociate and encourage the development of the story.

The practices that seem to complicate and derail the storytelling are primarily the questions asked by the friends. Some of the questions are understanding checks (lines 12 and 60), which deal with a potential comprehension 


\section{Jan Svennevig and Heidi E. Hamilton}

problem and thereby merely create a temporary suspension of the storytelling. But others, like the ones in lines 24,31 , and 32, topicalize matters that are recognizably not part of the storyline and thus propose a gradual topic shift to ancillary matters. These questions have the effect of derailing the storytelling in course. Also Gary's code-switching to Norwegian when renewing his story prompt seems to complicate the task, as Laura repeatedly switches back to her stronger language, English. For multilinguals with dementia, using their full linguistic repertoire may enhance their participation in communication (Svennevig et al. 2019).

\section{5 "We Used to Be the Caregivers": New Story Told Within an Institutional Setting}

The second case comes from the final few minutes of a one-hour weekly early-memory-loss support-group meeting in an urban recreational center in the northeastern United States. In contrast to the case examined above - in which a woman with dementia is prompted by her husband to tell a story known to both of them to friends over lunch in her home - in this excerpt, we follow the trajectory of a single utterance "we used to be the caregivers" (line 19) as it emerges and unfolds into a story within this institutional interaction involving seven individuals with dementia (although most do not contribute verbally to this excerpt), two experienced and professionally trained support-group facilitators, Abby and Nadine, and a visiting expert in dementia care, Mary.

Most of the hour-long interaction comprises extensive turns-at-talk by five support-group members as they respond to Mary's question "What is it like for you all to live with Alzheimer's (.) dementia (.) memory loss?" In these turns, members provide vivid and multi-faceted insights into their lived experiences with memory loss, touching on stigma and embarrassment, associated changes within the family, and descriptions of activities that bring joy into their daily lives.

Leading up to the excerpt below, facilitator Abby shifted the discourse topic away from this focus on PWDs towards a description of facilitators' work with PWDs' family members to help visitor Mary understand additional facets of dementia care at the recreational center. In lines 1-2, facilitator Nadine summarizes this aspect of their work for Mary while deftly including group members as addressees through her shift in pronoun use from third person to second person (i.e., by referring to "you guys" and "your families" in line 2; note the contrast to Abby's use of "their caregivers" in line 3).

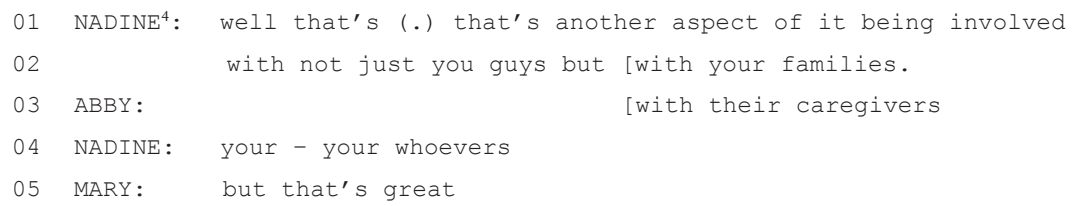




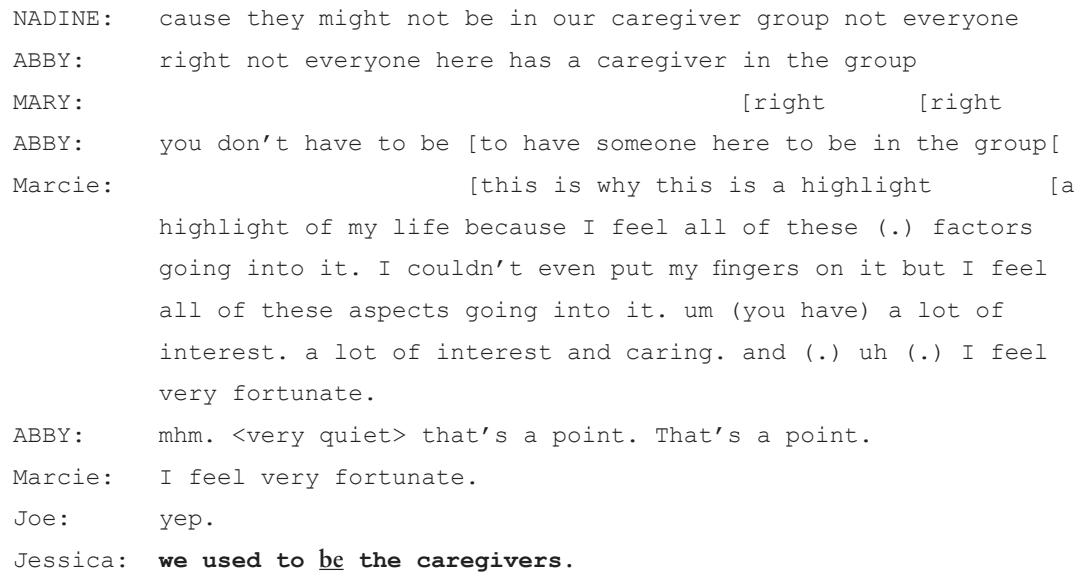

As Abby, Nadine, and Mary work toward topic closure by clarifying the connections and distinctions between the two groups with which they work, Nadine introduces the term "caregiver group" in line 6 (perhaps picking up on Abby's use of the term "caregivers" in line 3 that overlaps her own term "families" in line 2). At this point, group member Marcie takes the initiative to reenter the discussion by drawing a link ("this is why" in line 10) between her strong positive stance toward the support group ("highlight of my life" in lines 10-11; "I feel very fortunate" in lines 14-15 and 17) and how the facilitators had described their work ("all of these factors" in line 11; "all of these aspects" in lines 13) to Mary. Following validating turns by facilitator Abby and support-group member Joe, Jessica introduces a comment in the form of an unadorned assertion about how things had been in the past: "we used to be the caregivers" (line 19). This utterance displays a rather high degree of conversational agency. First, it reintroduces the topic of caregivers after a sequence that had moved the discussion in a different direction. Second, it recontextualizes it by shifting the focus from the group members' role as recipients of care to their role as providers of care (note the contrastive stress on "be").

Given that the comment is uttered at very low volume and lacks specific cohesive ties to what Marcie had just said, Jessica's utterance could quite easily have been "left out to dry," resulting in what frequently happens when PWDs attempt to enter fruitfully and fully into ongoing multiparty interactions. In this instance, however, fellow group member Marcie keeps Jessica's contribution alive by initiating a brief repair sequence (lines 20-21).

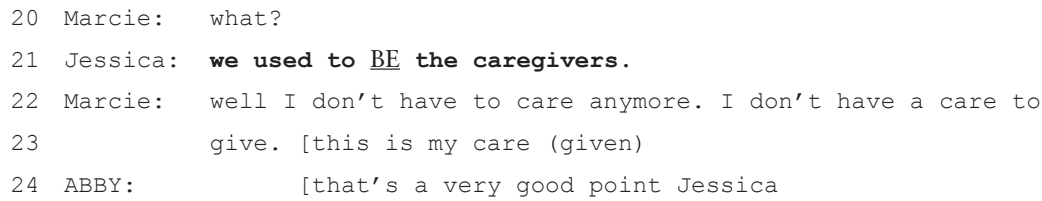




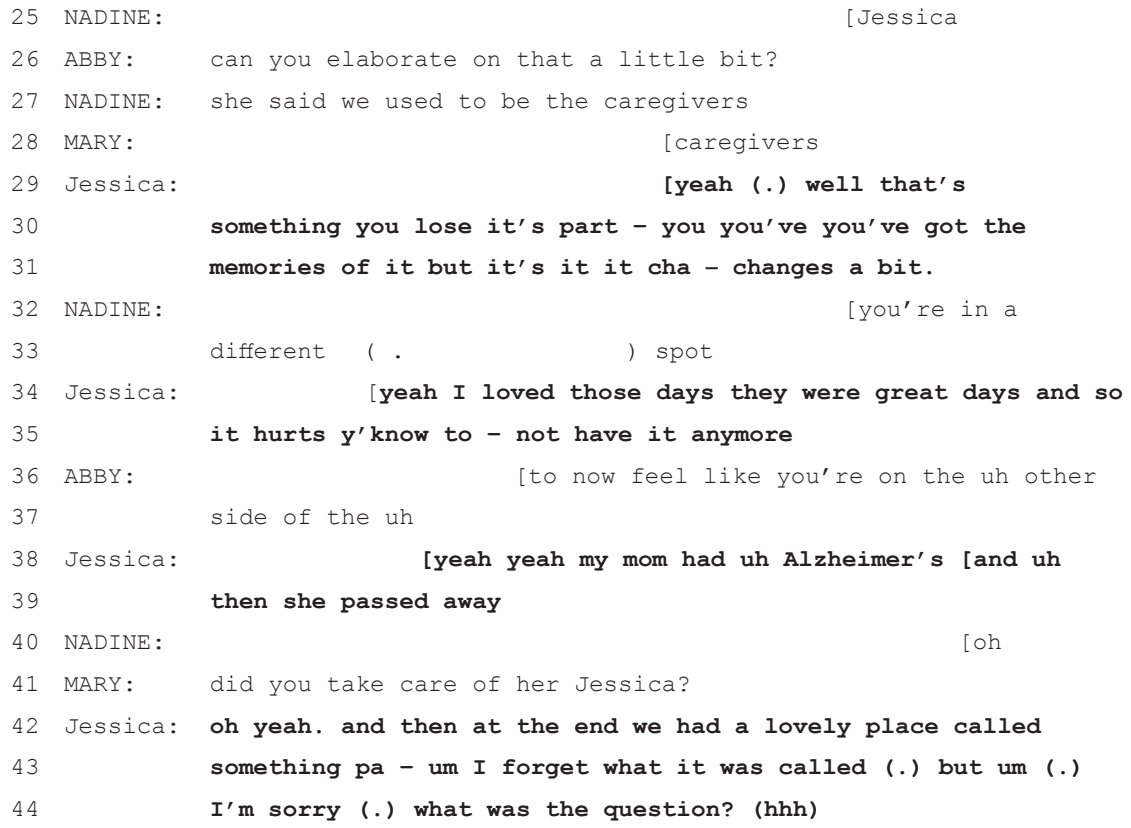

Jessica's subsequently repeated comment (this time loud enough for all to hear) then launches two different discourse trajectories at a figurative "fork in the road": 1) the taking up of a personal stance toward what Jessica just said, as indicated in lines 22-23 by Marcie's relatively dismissive stance; and 2) a narrative orientation toward Jessica's comment, as indicated by Abby's validating comment (line 24) and subsequent request for elaboration ${ }^{5}$ (line 26). Underlying Abby's moves is a recognition that Jessica's comment has refocused the discourse topic from matters related to the facilitators' here-and-now experiences to matters related to support-group members' there-and-then experiences - and that a story may indeed be lurking under the surface of the conversation.

The turns that immediately follow are highly consequential for the development (or not) of Jessica's story. Imagine if others had responded explicitly to Marcie's comments or if they had followed her lead in providing their own personal perspectives on having served as caregivers in the past. Instead, Nadine and Mary respond more immediately than Jessica herself to Abby's request, albeit (interestingly) simply repeating without any elaboration what Jessica had originally said (lines 27-28). Before their turns come to possible completion points, Jessica overlaps their talk and reenters the discussion (lines 29-31) to provide her elaboration. Although the language she uses to convey additional insights to Abby contains evidence of communicative challenges associated with dementia (e.g., semantically weak lexical items such as "something" and "part," repetition, and self-repairs; see Rochon, Leonard, and Goral 2018; Hamilton 2019), several clues in her 
language choices ("lose," "you've got the memories of it," and "changes a bit") appear to provide topical hooks related to change over time that others could build upon, as evidenced by Nadine's proffered reformulation in line 32-33.

Consistent with the approach highlighted above, in what follows we observe that Jessica's single "fleeting" utterance ("we used to be the caregivers") is recognized as a "tellable" (Sacks 1992) that could be transformed into a story - and that interlocutors hold the key through their vigilant attention to the narrative possibilities in what their partner is saying. In lines 34-35, Jessica begins to fill in elements of the distant world underlying the story by conveying a strong positive affective stance toward that time ("I loved those days"; "They were great days"), contrasting her life in the hereand-now with her life in the there-and-then through poignant language ("it hurts y'know to - not to have it anymore"). At this point in the unfolding of the storytelling process, group members learn more about Jessica as a figure in that distant world, but are not yet aware of other foundational building blocks of that world; e.g., for whom she was a caregiver; what actions she performed as a caregiver; and where and when she carried out her caregiving responsibilities.

Abby enters the discussion during Jessica's pause ("it hurts y'know to - ") in line 35 in an apparent attempt to finish Jessica's thought as to what "hurts," although their overlapping continuations in lines 35-36 display slightly different understandings of the contrasting feelings ("to not have it anymore" vs. "to now feel like you're on the uh other side of the uh"). In lines 38-39, then, Jessica latches onto Abby's proposal to divulge the core of the story: "my mom had uh Alzheimer's and uh then she passed away." And, although Jessica does not explicitly tie this utterance about her mother's health to her own role as "caregiver," Mary provides her with the opportunity to make this connection via her question in line 41 ("did you take care of her Jessica?"). In lines 42-44, then, Jessica begins to fill in temporal and spatial details of the world in which she was a "caregiver": focusing "at the end" of her mother's life where she had lived in "a lovely place." As Jessica attempts to provide greater specificity to her account, she runs into two kinds of memory challenges: 1) not being able to recall the name of the "lovely place" ("I forget what it was called"); and 2) forgetting the question that Mary had just posed a few seconds earlier ("I'm sorry (.) what was the question?").

It is in response to Jessica's interactional troubles that we note Mary's high degree of professional training and skillful sensitivity. Rather than, say, (meta)commenting on Jessica's forgetfulness, in lines 45-46 Mary simply repeats her earlier question with two changes: 1) a greater level of semantic precision, replacing the pronoun "her" in line 41 with "your mother" in lines 45-46; and 2) shifting from an unmarked yes-no question to an assertion that assumes the underlying proposition to be the case. 


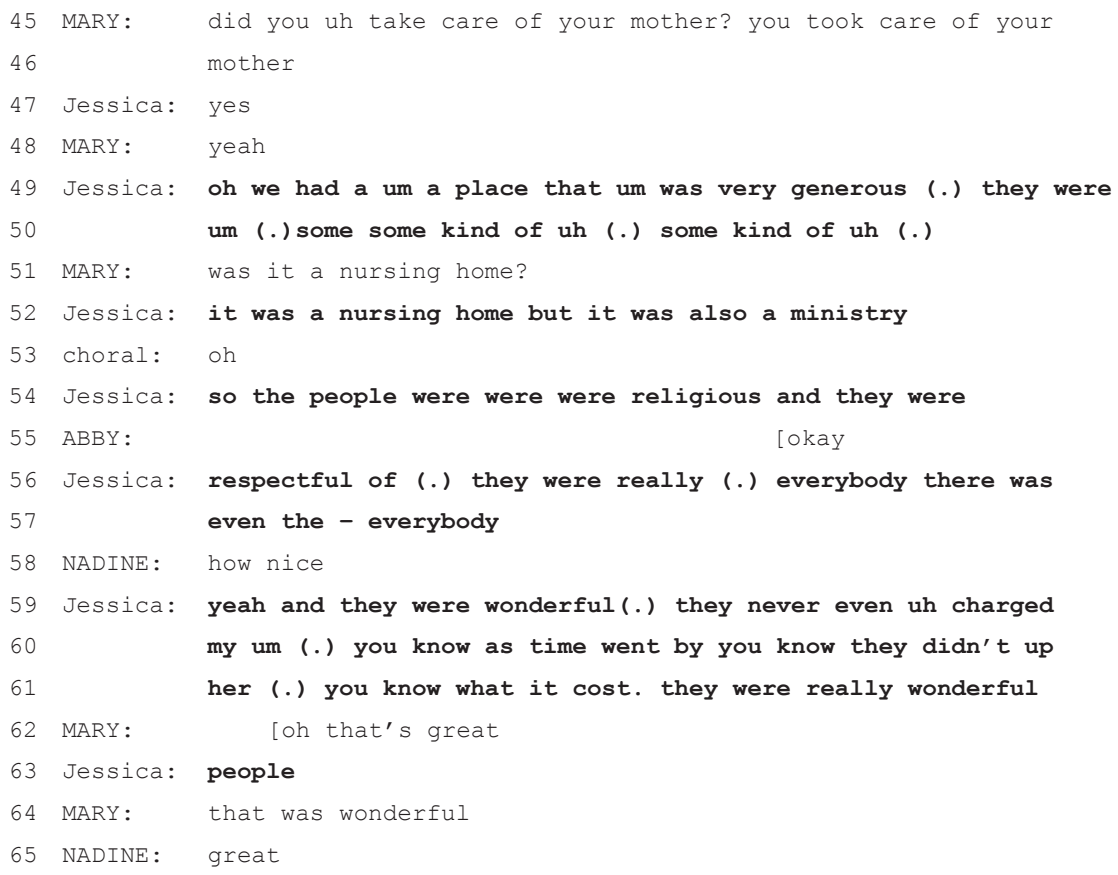

Unfortunately, Jessica's second attempt to respond to Mary does not extend much further than her first attempt; in line 49 interlocutors learn only that the "place" was not only "lovely" but also "generous" before Jessica runs into another word-finding challenge, when she tries to characterize the "kind of place" it was ("they were um some some kind of uh some kind of uh.."). Again, it is Mary who steps in in line 51 with a candidate answer ("was it a nursing home?").

This scaffolding strategy appears to assist Jessica, who not only repeats the offered lexical item but provides the additional detail that it was "also a ministry" (line 52) followed by other associated details regarding the people who populated the "place"; i.e., that they were "religious" (line 54), "respectful" (line 56), and "wonderful" (lines 59, 61). In lines 59-61, interlocutors begin to learn more about the kinds of actions that would have led Jessica to assert in line 49 that the "place" was "generous"; although Jessica's communicative challenges again get in the way of understanding explicitly what these actions were, lines 59-61 contain enough clues to help interlocutors infer that Jessica's very positive view of the place where her mother lived had to do with how the institution dealt with the fee structure for her mother's care ("they never even charged my um (.) you know as time went by you know they didn't up her (.) you know what it cost”). In lines 62-65, then, the topic of "Jessica as caregiver" winds down as Mary and Nadine align with Jessica in their positive stance toward the situation Jessica has been describing. 
Similar to the "dinner rolls" story above, what we observe in this extract is a PWD, Jessica, who manages to contribute to a multiparty discussion with the assistance of others in the interaction. In contrast to the "dinner rolls" story that was introduced by the spouse of the PWD, in this case Jessica introduces within a larger discussion of dementia caregiving a single poignant and relevant observation that, although the individuals in the support group are currently care receivers, they have themselves been caregivers in the past. ${ }^{6}$ Again, in contrast to the "dinner rolls" story, no one in the support-group interaction shared the specific epistemic domain that Jessica was referencing; all needed to draw on a variety of strategies to bring to the surface what underlay the comment.

The initial heavy lifting to launch the story was carried out by group facilitator Abby who oriented to Jessica's single comment as a portal to an underlying experience that had the potential to become a more expansive story as the discussion ensued. By selecting Jessica's comment (rather than, say, Marcie's response to Jessica in lines 22-23) as worthy of continued attention (lines 24, 26), Abby opened up a space for Jessica to continue to talk, sparking subsequent reformulations of her talk by Nadine (line 32-33) and Abby (lines 36-37). Once the heart of Jessica's story emerged, Mary then stepped in to build explicit connections across Jessica's utterances (line 41 ), to maintain a sense of normalcy in the display of memory loss (line 45-46) and to aid in a word search (line 51). And in efforts to make collective sense of the story and to move it toward closure, Abby, Nadine, and Mary all took up epistemic (lines 53, 55) and affective stances (lines 58, 62, $64,65)$ toward aspects of Jessica's story.

With the assistance of these others, Jessica drew on her own specific experiences as an adult daughter caring for her own mother to make a more general point - "we used to be the caregivers" - within a wide-ranging discussion carried out primarily by dementia care professionals within an institutional setting. That said, as we follow the trajectory of the initial voicing of "we used to be the caregivers" in line 19 through the concluding stance-taking to elements of the story in lines 64-65, it is important to recognize the limits of even these well-intentioned discursive efforts of Jessica's interlocutors. Despite these professionals' strategies to support Jessica's contributions to the multiparty discussion, the resulting story that emerges within the interaction contains very little detail: interlocutors come away with a generally uplifting sense that she enjoyed her caregiving experiences within a wonderful residential facility in which her mother lived out the end of her life.

\section{Discussion}

In his multi-faceted and insightful examination of collaborative storytelling in dementia, Hydén provides the following characterization of the link 
between dementia-related communicative and cognitive changes and PWDs' sense of self:

These challenges and changes result in new relationships between the person, his or her body, the world, and other persons. In particular, the person with dementia moves from an experience of being a relatively autonomous individual, to becoming dependent on others in a shrinking world, and with a waning sense of agency.

(Hydén 2018, 176)

It is this trajectory from relative autonomy to interdependence with others that has centered our theoretical and analytical attention on storytelling within multiparty casual and institutional interactions. As we examined how Laura and Jessica recounted family stories from times past that related in clear ways to their lunchtime and support-group interactions in the hereand-now, we hope to contribute to a more nuanced understanding of "how persons tell stories together and in what ways the presence of dementia alters how persons collaborate" (Hydén 2018, 117).

Despite the differences in storytelling situations represented in the two cases analyzed above, we note that Laura's and Jessica's interlocutors made use of similar discursive practices to support their contributions to multiparty interactions:

1) inviting a contribution from a PWD (e.g., How was it with your father's eh family, Laura, you can tell about ...)

2) amplifying/reformulating a PWD's utterance (e.g., You're in a different (.) $s p o t$ )

3) asking a PWD to clarify or elaborate on a previous utterance (e.g., That's a very good point, Jessica.. can you elaborate on that a little bit?)

4) asking a clarification or follow-up question (e.g., Did you ub take care of her, Jessica?)

5) assisting with a PWD's word search (e.g., Was it a nursing home?)

6) repeating a previous utterance without calling explicit attention to the memory gap when the PWD indicates it has been forgotten (e.g., but how was it when they sat down at the table - eh Laura, have you told about the dinner table?)

7) evaluating/taking up a stance toward the PWD's contribution (e.g., That was wonderful)

In addition to these active strategies, interlocutors in both our cases also helped to create an environment conducive to verbal contributions by the PWD by "doing nothing" verbally; i.e., by holding off on the taking of a turn-at-talk even in the face of long pauses. By remaining silent (e.g., resisting the proposal of a "missing" word or attempting to complete the PWD's clause), these interlocutors provided discursive space to the PWDs to 
continue to talk, even if that subsequent talk displayed evidence of the influence of dementia (e.g., extended pauses and repetitions). They also encouraged the storytelling by producing continuers, assessments (ob dear) and other displays of interest and attention, such as laughter and nods.

The analysis has also identified practices that may hamper or derail the storytelling. In the first case, it was a request for elaboration on the background information provided in the story that expanded the insertion sequence to a point where it became difficult for the PWD to resume the story. Furthermore, the husband's code-switching to her weaker language, Norwegian, may have complicated the task for her. In the second case, it was a self-oriented comment by an interlocutor who did not orient to the story potential of the topic and could quite easily have derailed the story before it had a chance to be launched. We note then that interlocutors' contributions that do not directly support and encourage the storytelling in progress may lead to missed opportunities for storytelling or to stories being abandoned and derailed along the way.

The two cases reveal several kinds of opportunities and challenges that may arise in these different types of settings. In cases where the PWDs are in the company of spouses or others with whom they share considerable personal background knowledge, interlocutors may identify opportunities for the PWD to tell a story and elicit it by producing a story prompt. The danger here is that spouses may be tempted to tell the story for the PWD, given their epistemic access to it and the likelihood that they would be able to tell it more fluently. So in relation to such situations, our case illustrates a spouse who consistently orients to the PWD's right and obligation to tell the story despite her apparent difficulties in carrying out the task. Within institutional settings, where conversation partners typically do not typically know a great deal about each other's lives, it may be difficult for these interlocutors to identify opportunities for the PWD to tell personal stories. Our case demonstrates professionally trained facilitators skillfully identifying the story potential behind a conversational contribution that was not itself presented as a story preface and subsequently eliciting a personal narrative by requesting elaboration and asking topicalizing questions.

Our findings suggest ways in which interlocutors' discursive strategies may be used to promote personal agency and feelings of well-being in PWDs who are managing symptoms related to memory and language. Interactional moves that encourage PWDs to launch and tell their stories within complex multiparty interactions may thereby not only help PWDs to display their own personal authoritative knowledge in the conversation, but - by serving to spark memories of historical and personal relevance in others in the group - may ultimately construct a more integrated and inclusive environment for all parties. These supportive topic-building strategies may, then, creatively and compassionately lead to heightened levels of well-being for PWDs, as they discern that their individual expression is connected to enhanced social interaction more generally. 


\section{Jan Svennevig and Heidi E. Hamilton}

\section{Acknowledgements}

We would like to thank the members of the research network Dementia, Language, Interaction and Cognition (DELIC) for discussions on the data used here, and especially Elin Nilsson and Anna Ekström for useful comments on a draft of the article. This work was partly financed by the Research Council of Norway through its Centres of Excellence funding scheme, project number 223265, as well as project number 250093 (JS) and supported by a Humboldt Research Award (HH).

\section{Notes}

1 The transcriptions comply to standard CA conventions (Jefferson 2004). Laughter quality is indicated by $£$ signs before and after the words or sounds concerned.

2 Here and other places in the story, Laura seems to have some problems with referential expressions. In this case, she seems to be talking about her grandfather and not her father, probably alluding to his fertility.

3 Again, Laura seems to have some reference problems in that she refers to "we" rather than "they."

4 In this and subsequent excerpts, names of support group facilitators and the visiting expert in dementia care are spelled in all capital letters to contrast with names of individuals with dementia.

5 Note that Abby does not build on Marcie's comments in lines 22-23 but instead reaches back to Jessica's stand-alone comment in line 19.

6 In order to begin to fathom the degree of courage this interactional move required of Jessica, it is important to "listen in on" Jessica's contribution to Mary's understanding of life with dementia much earlier in the hour-long meeting when she related her reluctance to talk: "I have memory loss and um it's it's embarrassing you know when (.) when you're telling somebody something and (.) I forget names (.) and you forget the person's name after you've given the whole synopsis of what you know they mean to you."

\section{References}

Backhaus, P. 2018. "Reclaiming Agency in Resident-Staff Interaction: A Case Study from a Japanese Eldercare Facility.” Discourse Studies 20: 205-220.

Crichton, J. and T. Koch. 2011. "Narrative, Identity and Care: Joint Problematisation in a Study of People Living with Dementia.” In Discourses of Deficit, edited by C. Candlin and J. Crichton, 101-118. Basingstoke: Palgrave Macmillan.

Goodwin, M.H. 1997. "By-play: Negotiating Evaluation in Storytelling.” In Towards A Social Science of Language, edited by G.R. Guy, C. Feagin, D. Schiffrin, and J. Baugh, 77-102. Amsterdam/Philadelphia: Benjamins.

Gumperz, J. 2015. "Interactional Sociolinguistics: A Personal Perspective." In Handbook of Discourse Analysis, 2nd edition, edited by D. Tannen, H.E. Hamilton and D. Schiffrin, 309-323. Oxford: John Wiley \& Sons, Inc.

Hamilton, H.E. 2008. "Narrative as Snapshot: Glimpses into the Past in Alzheimer's Discourse." Narrative Inquiry 18: 53-82.

Hamilton, H.E. 2019. Language, Dementia and Meaning Making. Cham: Palgrave Macmillan. 
Hamilton, H.E. and M. Baffy. 2014. "Preparing for Performance: Identity Shaping through Script Editing in an Early Memory Loss Support Group.” In Dialogue and Dementia: Cognitive and Communicative Resources for Engagement, edited by R. Schrauf and N. Müller, 213-244. New York: Psychology Press.

Heritage, J. 2012. "Epistemics in Action: Action Formation and Territories of Knowledge." Research on Language and Social Interaction 45: 1-29.

Hydén L.-C. 2011. "Narrative Collaboration and Scaffolding in Dementia.” Journal of Aging Studies 25: 339-347.

Hydén L.-C. 2018. Entangled Narratives: Collaborative Storytelling and the Re-imagining of Dementia. New York: Oxford University Press.

Hydén, L.C. and L. Örulv. 2009. "Narrative and Identity in Alzheimer's Disease: A Case Study.” Journal of Aging Studies 23: 205-214.

Jefferson, G. 2004. "Glossary of Transcript Symbols with an Introduction." In Conversation Analysis. Studies from the First Generation, edited by G. Lerner, 13-34. Amsterdam: Benjamins.

Kitwood, T. 1997. Dementia Reconsidered: The Person Comes First. Berkshire: Open University Press.

Landmark, A.M. and J. Svennevig. forthcoming. "Initiating and Pursuing a Topical Agenda with Limited Communicative Resources.” In Interaction and Dementia: From Diagnosis to Daily Discourse, edited by P. Muntigl, D. Jones and C. Plejert. Cambridge: Cambridge University Press.

Leibing, A. 2006. "Divided Gazes: Alzheimer's Disease, the Person within, and Death in Life." In Thinking about Dementia: Culture, Loss, and the Anthropology of Senility, edited by A. Leibing and L. Cohen, 240-268. New Brunswick: Rutgers University Press.

Lerner, G.H. 1992. “Assisted Storytelling: Deploying Shared Knowledge as a Practical Matter.” Qualitative Sociology 15: 247-271.

Mandelbaum, J. 1989. "Interpersonal Activities in Conversational Storytelling." Western Journal of Speech Communication 53: 114-126.

Mazeland, H. and M. Huiskes. 2001. "Dutch 'But' as a Sequential Conjunction: Its Use as a Resumption Marker." In Studies in Interactional Linguistics, edited by M. Selting and E. Couper-Kuhlen, 141-169. Amsterdam: John Benjamins.

Nilsson, E. 2017. "Fishing for Answers: Couples Living with Dementia Managing Trouble with Recollection." Educational Gerontology 43: 73-88.

Nilsson, E., A. Ekström, and A.R. Majlesi. 2018. "Speaking for and About a Spouse with Dementia: A Matter of Inclusion or Exclusion?” Discourse Studies 20: 770-791.

Österholm, J.H. and C. Samuelsson. 2015. "Orally Positioning Persons with Dementia in Assessment Meetings." Ageing \& Society 35: 367-388.

Ottesjö, C. 2005. "Iallafall som diskursmarkör [Anyway as a Discourse Marker]." In Samtal och grammatik. Studier I svenskt samtalsspråk. [Conversation and Grammar. Studies in Swedish Conversational Language], edited by J. Anward and B. Nordberg, 201-230. Lund: Studentlitteratur.

Ramanathan, V. 1995. “Narrative Well-formedness in Alzheimer's Disease: An Interactional Analysis Across Settings." Journal of Pragmatics 23: 395-419.

Raymond, G. and J. Heritage. 2006. "The Epistemics of Social Relationships: Owning Grandchildren." Language in Society 35: 677-705.

Rochon, E., C. Leonard, and M. Goral. 2018. "Speech and Language Production in Alzheimer's Disease." Aphasiology 32: 1-3. 


\section{Jan Svennevig and Heidi E. Hamilton}

Sacks, H. 1992. Lectures on Conversation, edited by G. Jefferson. Oxford: Blackwell. Sacks, H. 1974. "Some Considerations of a Story Told in Ordinary Conversation." Poetics 15: 127-138.

Schegloff, E.A. 2007. Sequence Organization in Interaction. A Primer in Conversation Analysis. Cambridge: Cambridge University Press.

Schrauf, R. and N. Müller, eds. 2014. Dialogue and Dementia: Cognitive and Communicative Resources for Engagement. New York: Psychology Press.

Selting, M. 2017. "The Display and Management of Affectivity in Climaxes of Amusing Stories. Journal of Pragmatics 111: 1-32.

Seuren, L.M., M. Huiskes, and T. Koole. 2016. "Remembering and Understanding with Oh-prefaced Yes/No Declaratives in Dutch." Journal of Pragmatics 104: 180-192.

Shenk, D. 2005. "There was an Old Woman: Maintenance of Identity by People with Alzheimer's Dementia." In Alzheimer Talk, Text, and Context, edited by B.H. Davis, 3-17. New York: Palgrave.

Sidnell, J. and T. Stivers, eds. 2013. The Handbook of Conversation Analysis. Oxford: Wiley-Blackwell.

Stivers, T. 2008. "Stance, Alignment, and Affiliation During Storytelling: When Nodding Is a Token of Affiliation." Research on Language \& Social Interaction 41: 31-57.

Svennevig, J., P. Hansen, H.G. Simonsen, and A.M. Landmark. 2019. "Codeswitching in Multilinguals with Dementia: Patterns across Speech Contexts." Clinical Linguistics \& Phonetics 10-11: 1009-1030. doi:10.1080/02699206.2 019.1600170

Williams, V., J. Webb, S. Dowling, and M. Gall. 2019. "Direct and Indirect Ways of Managing Epistemic Asymmetries when Eliciting Memories.” Discourse Studies 21: 199-215.

Wray, A. 2020. The Dynamics of Dementia Communication. Oxford: Oxford University Press. 


\title{
9 Assessment Challenges in Acquired Aphasia in Multilingual Individuals
}

\author{
Monica Norvik and Mira Goral
}

\section{Introduction}

Multilingualism is the rule, rather than the exception, around the world (e.g. Grosjean 2013).

Multilingual adults may experience a mild or substantial loss of one or more of their languages either because they stop using the language due to changes in their sociolinguistic context, or as a result of an acquired impairment. One source of language impairment in adults is aphasia, an acquired language disorder that results from a focal brain lesion. Assessment of all languages of multilingual individuals with aphasia is critical for accurate diagnosis and for treatment planning. Multilingual assessment, however, poses challenges. In this chapter, we will discuss and illustrate practices and challenges in the assessment of linguistic and communication skills in multilingual individuals who acquire aphasia.

\subsection{Multilingual Individuals}

Multilingual people are those who use more than one language in everyday life. They may live in broader or narrower multilingual sociolinguistic contexts. Broad multilingual sociolinguistic contexts include multilingual countries (e.g. India, Belgium, Brazil, Singapore, South Africa) and cities (e.g. Lebanon, Montreal, Brussels), where several languages are spoken as the official (and majority) languages. In such sociolinguistic environments, individuals may grow up speaking more than one language. Individuals who grow up in monolingual sociolinguistic environments, in contrast, typically grow up speaking one language, but may become multilingual when they move to a place where another language is spoken. People who migrate might live completely immersed in the majority language of the new environment or might live in a community of speakers of their first language (L1), the minority language, with varying degrees of exposure to the majority language, their second language (L2). The exposure to and use of an L2 have a direct impact on the levels of L2 proficiency attained. Most typically, individuals who are immersed in their L2 develop higher proficiency levels 
in that language than those who have greater exposure to their L1 and only limited interactions in their L2.

Language exposure and use greatly affect the level of an individual's language proficiency. Proficiency may also depend on the age and manner of acquiring the languages, attitudes toward each of the languages, and the linguistic relations among the relevant languages. Moreover, multilingualism is a dynamic phenomenon. Users of multiple languages rarely have a stable proficiency in all their languages nor a fixed communication environment across their lifespans. Moreover, the languages of multilingual speakers mutually influence one another. Establishing normative data for the purpose of assessing linguistic abilities for this heterogenic group of speakers is all but impossible.

\subsection{Aphasia}

The most common aetiology of aphasia is a stroke in the language-dominant hemisphere (the left hemisphere in most individuals). A stroke typically results from a blockage of a blood vessel due to a blood clot in the brain (ischaemic stroke) or from a rupture of a blood vessel in the brain (haemorrhage) (Papathanasiou, Coppens, and Davidson 2017). Strokes are the second most common cause of death globally, and around half of all stroke survivors live with disability; around $30 \%$ of all stroke survivors acquire aphasia as a consequence (Flowers et al. 2013). Aphasia may also be caused by traumatic brain injury or brain tumour. People who acquire aphasia experience varying degrees of impairment in the various linguistic and functional aspects of communication. Difficulties can manifest in all or some language modalities (e.g. comprehension of spoken language, speaking, reading, and writing) and in all or selected linguistic domains (e.g. syntax, phonology). A hallmark deficit associated with aphasia is anomia, difficulty retrieving words during language production. In addition to these language deficits, people with aphasia can also experience speech difficulties, including apraxia of speech, which impairs the programming of motor speech production, and dysarthria, which is a speech impairment due to weakness of the articulators. Language and communication impairments range from mild to moderate to severe; differing degrees of deficits in language comprehension and production may interfere minimally with daily communication or can lead to severe communication restrictions. These communication difficulties may result in decreased self-confidence, restricted social interaction, loss of employment, or social isolation.

\subsection{Language Assessment of Individuals With Aphasia}

Assessment in aphasia involves the gathering of quantitative and qualitative data about the person's abilities to understand and produce language and to communicate, as well, about restrictions that the acquired language 
impairments impose on communicative activities and life participation (Murray and Coppens 2017). An individual selection of assessment tools and approaches is usually made while the previous and the current levels of functioning, as well as the aims and needs of the individual with aphasia, are considered. Additionally, for multilingual speakers, one aim of assessment is to discover the preserved and impaired communication abilities in each language (Ansaldo et al. 2008). Language assessment of speakers with aphasia can be formal (with standardised and norm-referenced tests) or informal (e.g. creating and manipulating stimuli to make clinical decisions, engaging in semi-spontaneous conversation, as well as gathering premorbid language information), and often the approaches are combined.

In addition to the linguistic factors - that is, specific features of each of the languages under consideration - there are non-linguistic factors that are unique for multilingual speakers with aphasia. To supplement the background information that is normally collected when working with people with aphasia (e.g. information on educational, medical, occupational, social background), it is imperative to start with an assessment of the premorbid language history of the multilingual speaker. This refers to how and when the languages were acquired, and domains and frequency of language use. Such information is important to detect premorbid language dominance and mastery, and the degree of use of the different language modalities. This can be done as a self-report by the multilingual speaker him/herself, and/or with assistance from significant others (Centeno and Ansaldo 2013; Paradis and Libben 1987; Roberts 2008; Roberts and Kiran 2007).

There are several instruments that have been developed to diagnose and characterise aphasia, and to assess the degree of aphasia severity. Aphasia batteries, such as the Boston Aphasia Diagnostic Examination (Goodglass, Kaplan, and Barresi 2000), the Western Aphasia Battery Revised (Kertesz 2006), and the Aachen Aphasia Test (Graetz, De Bleser, and Willmes 1992) have been developed in the context of the syndrome approach to aphasia classification, which attempts to classify people with aphasia into aphasia types that share clusters of characteristics. For example, a person with relatively intact comprehension abilities, marked expressive language difficulties (including reduced utterance length, impaired grammatical structure, effortful speech production), and impaired repetition abilities, may be classified as having Broca's aphasia. Such batteries have been developed in several languages and have been translated or adapted to other languages (e.g. the Aachen in Italian; the WAB in Korean). Other approaches to aphasia classification include the psycholinguistic approach, which yielded the Psycholinguistic Assessments of Language Processing in Aphasia (PALPA; Kay, Lesser, and Coltheart 1992). Here the goal is to assess a range of linguistic abilities and to characterise the aphasia in terms of areas of linguistic strength and weaknesses. The PALPA was developed in English and has been adapted to several other languages (e.g. Spanish, Hebrew, Norwegian, Japanese). The Comprehensive Aphasia Test (CAT; Swinburn, 
Porter, and Howard 2004) is another battery that is based on the psycholinguistic approach. The CAT was developed in English and has now been adapted into multiple other languages (e.g. Norwegian, Dutch, Croatian), with a deliberate attempt to retain its psycholinguistic features (e.g. word frequency, word length) (e.g. Fyndanis et al. 2017; Kuvač Kraljević, Matić, and Lice 2020).

In addition to comprehensive batteries, tests of specific language abilities have been developed for aphasia. These include tests of reading abilities (e.g. the Reading Comprehension Battery for Aphasia, RCBA; Lapointe and Horner 1998), of picture naming (e.g. the Boston Naming Test (BNT; Kaplan, Goodglass, and Weintraub 2001)), and of verb and sentence processing and production (e.g. Northwestern Assessment of Verbs and Sentences (NAVS; Thompson 2012)). These tests have been developed primarily in English and some have been translated or adapted to other languages (e.g. BNT in Spanish). A challenge in adapting such tests to languages other than the one in which they were developed is maintaining psycholinguistic variables and levels of difficulty.

Finally, specific tests that are designed to measure the specific abilities that are targeted in treatment in aphasia are often developed in an attempt to capture treatment-related change. For example, a list of trained items, related untrained items, and unrelated items may be developed to assess treatment outcome of a treatment that targets specific lexical items (e.g. Edmonds and Kiran 2006).

There are normative data for the formal tests that are available for assessing people with aphasia. These data are often based on relatively small numbers of participants. Furthermore, all normative data for these tests are gathered from monolingual individuals. As well, when tests were adapted from one language to another, little attention has been given to making the versions in the different languages comparable, as the intention was not to test several languages within an individual. A few aphasia tests, however, have been designed with the aim of testing aphasia in multilingual people. The most used one is the Bilingual Aphasia Test (BAT; Paradis and Libben 1987). The BAT has been developed in over 70 languages and aims to provide a comparable measure in multiple languages (https://www.mc gill.ca/linguistics/research/bat\#ebat). It includes a section for collecting language background history, one for assessing a variety of language abilities in each language, and a section on translating abilities from one language to another. Challenges associated with the BAT include less than ideal items when adapted from English to other languages, minimal testing of certain linguistic aspects that are less relevant in English (e.g. morphology), and culturally inappropriate items and tasks, despite the authors' best effort to adapt rather than translate the test.

Formal assessment in aphasia may underestimate the person's linguistic and communication abilities due to the formal setting, the effect of anxiety on performance, and issues of fatigue and decreased attention. Informal 
assessment, such as free conversation between the examiner and the person with aphasia, may aid in completing the diagnostic process but may pose challenges of uniformity of assessment and of scoring. For example, people with aphasia may rely on their interlocutor in such situations and produce minimal output or avoid structures and items that are difficult for them to produce. Moreover, scoring systems for narrative and discourse production in aphasia have not been standardised to date.

Assessment of people with aphasia can be especially challenging when they are multilingual minority speakers and are multicultural. In addition to limited assessment tools, challenges include individual differences (e.g. in premorbid language histories), availability of multilingual clinicians, and the interpretation of multilingual assessment.

\section{Aphasia in Multilingual People}

Multilingual individuals who acquire aphasia often experience comparable levels of impairment in all their languages (e.g. Fabbro 2001; Kuzmina et al. 2019). Comparable degrees of impairment would mean that those people who were highly proficient in all their languages prior to the stroke demonstrate similar levels of aphasia severity in those languages and, similarly, individuals who were more dominant in one of their languages prior to the stroke will have better abilities in that language after the stroke (i.e. parallel patterns of impairment). Nevertheless, many cases have been reported of individuals for whom the relative impairment and recovery post-stroke is different from what might have been expected based on their pre-stroke relative proficiency (e.g. Paradis 2004). In some cases, only one language appears available and in other cases, some languages are more impaired than others (i.e. non-parallel impairment). Researchers have investigated the variables that might explain whether multilingual individuals with aphasia will demonstrate parallel or non-parallel patterns of impairments across their languages. These variables include age and manner of language learning, frequency and domain of language use, and language-specific characteristics (e.g. Goral 2015; Kuzmina et al. 2019).

\subsection{Case Illustrations}

We will illustrate the heterogeneity and the complexity associated with assessing multilingual people with aphasia by presenting two individuals with severe chronic non-fluent stroke-induced aphasia who participated in a treatment study at MultiLing in Oslo, Norway. One participant, P1, is a multilingual (Singhalese-Tamil-English-Norwegian) right-handed 59-yearold man who was 12 years-post-onset at the time of the study. He was born in Sri Lanka where he completed high school and an undergraduate degree. He had been living in Norway for 32 years. At the time of the study he reported using primarily Tamil and Norwegian. He was tested in Tamil, 
English, and Norwegian (Singhalese was not tested due to difficulty finding an interpreter and in accordance with the participant's wish not to be tested in a language that he rarely used).

The other participant, P2, is a multilingual (Farsi-English-Hindi-UrduPunjabi-Norwegian), right-handed 60-year-old man who was one-yearpost-onset at the time of the study. He was born in Iran, where he lived until he was 16, then he lived in Canada for four years, in England for one year, and in India for ten years before moving to Norway where he has been living for the past 30 years and where he also completed a master's degree. At the time of the study, he reported using primarily Norwegian and some Farsi. P2 was tested in Farsi, English, and Norwegian (the other languages were not tested in accordance with the participant's wish, due to minimal use).

Both participants had auditory comprehension deficits in addition to impairments in spoken production. P1 used both spoken and written language to communicate. Results from the BAT and object- and action-naming tasks in each of the three tested languages for each participant as well as the results from the Pyramids and Palm Trees (Howard and Patterson 1992) and the Kissing and Dancing (Bak and Hodges 2003) semantic processing tests are presented in Table 9.1.

As can be seen in Table 9.1, both participants had relatively preserved semantic processing (as measured by the action and object processing tests: the Kissing and Dancing and the Pyramids and Palm Trees tests). In contrast, their lexical retrieval abilities are severely compromised in all three languages, with a slightly better performance in object-naming in Norwegian for both participants. The BAT results, combining receptive and expressive abilities, also show moderate-severe impairment in all three languages.

To obtain these results, we needed to consider several variables and make multiple decisions to balance the scientific ideal on the one hand and clinical feasibility on the other. We will now turn to discuss each of the four challenges we listed above, namely, individual differences, multilingual clinicians and interpreters, appropriate assessment tools, and interpreting results, providing examples from the two cases described.

\section{Individual Differences}

\subsection{Obtaining Language Background}

To gather self-reported information, most researchers and clinicians use one of the existing language background questionnaires that have been designed to obtain information about history of language learning, history and frequency of language use, and self-rated proficiency (e.g. Muñoz, Marquardt, and Copeland 1999; Paradis and Libben 1987). For proficiency self-rating, people with aphasia may be asked to rate their pre- and postmorbid skills 


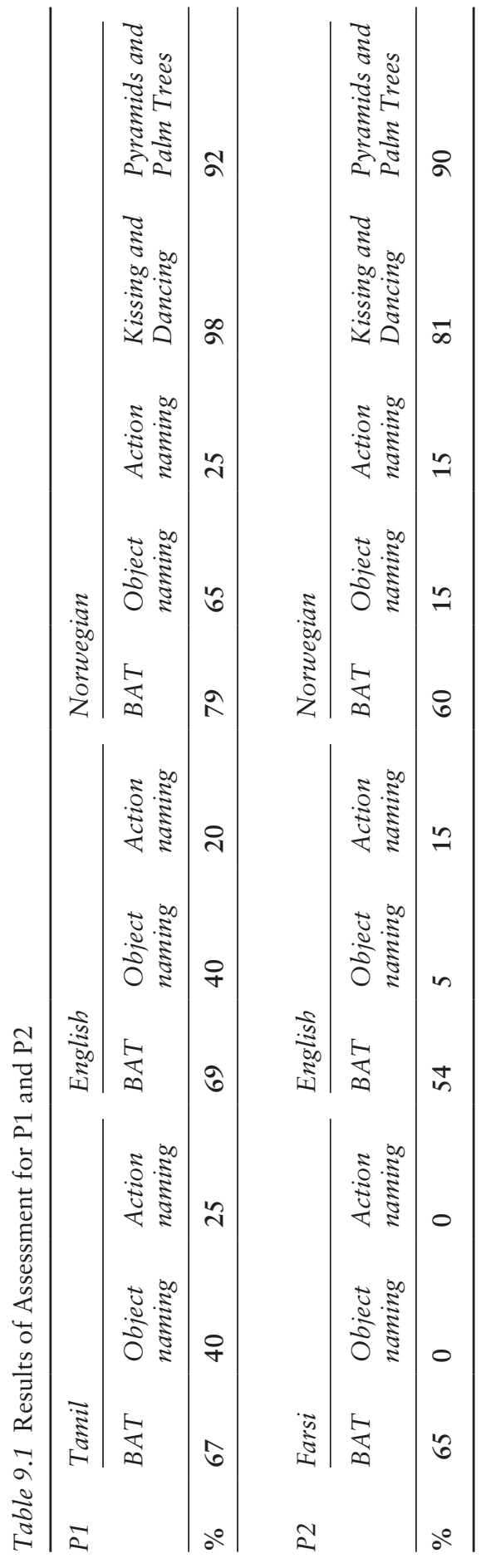


in various domains (e.g. speaking, writing) on a Likert scale (e.g. $0=$ none; $10=$ perfect $)$.

Self-rated proficiency has been used in studies with neurologically typical multilinguals as well, and the reliability of self-reports has been examined in several studies, revealing significant but moderate (at best) correlations between objective measures and self-ratings (e.g. Marian, Blumenfeld, and Kaushanskaya 2007). In a recent paper, however, Tomoschuk, Ferreira, and Gollan (2019) found that the self-rated proficiency was highly variable. This may be especially pronounced when comparing multilingual people with different language combinations, also within subgroups of multilingual individuals who speak the same languages but where there is variation in language history and language dominance. Self-reported bilingual language proficiency should thus be interpreted with caution, as it seem to vary in its reliability. Therefore, Tomoschuk et al. recommend objective measures, if available.

For example, our participant P1 (Singhalese-Tamil-English-Norwegian) reported with the LEAP-Q (Marian, Blumenfeld, and Kaushanskaya 2007) that his pre-stroke language proficiency was perfect (i.e. 10/10) in all his four languages (except reading and writing in Tamil, where he indicated a $5=$ adequate). It is not uncommon that, when people rate their language proficiency, they may rate their peak proficiency, that is, the highest level of proficiency they achieved in a given language. In multilingual individuals with aphasia, we cannot attribute postmorbid differences between the languages solely to brain damage; they may already have differed premorbid. For his post-stroke abilities, P1 rated his Norwegian at 3/10, Tamil at 2/10, and Singhalese and English at 1/10. These ratings, however, appear consistent with the results of our testing, as he scored slightly higher in Norwegian on the BAT and in object-naming (see Table 9.1).

In multilingual people with aphasia, it is impossible to assure the validity of self-ratings, in the absence of objective measurement of skills prior to the aphasia onset. Family members and caregivers are only occasionally asked to subjectively rate the individual with aphasia's abilities prior to the stroke, although informally they may be consulted for their input. As with self-ratings, there is also uncertainty regarding the reliability of family members' report on proficiency. Often the family members do not share all the languages of the person with aphasia, and their judgement of language proficiency may thus be based on their impression, which may not reflect the true proficiency of the language in question. To circumvent a possible misjudgement from the family, a more viable manner may be to ask family members and caregivers to subjectively assess the communication abilities, rather than language proficiency, of the person with aphasia following the stroke and, when intervention is administered, following the therapy (for example, by using the Communicative Effectiveness Index, CETI, Lomas et al. 1989). Here findings have demonstrated that whereas the correlation between the ratings completed by the person with aphasia 
and by their caregiver is typically significant, it is far from perfect, and that people with aphasia may rate themselves as more effective communicators or less severely impaired than how they are perceived by their caregivers (Rautakoski, Korpijaakko-Huuhka, and Klippi 2008). In many cases of multilingual persons, not only may minimal background information pose difficulty in establishing pre-aphasia proficiency, but also limited access to family members could limit the gathering of family-reported information.

To complicate matters more, the dynamic nature of proficiency among multilingual speakers presents an additional challenge. Multilingual persons who are immersed in one of their languages and use another of their languages with limited frequency may experience language attrition in the lessused language, that is, a deterioration of their linguistic and communicative abilities (e.g. Lerman, Goral, and Obler 2020; Schmid 2013). Changes associated with language attrition are often subtle in people who were adults before changing their language environment and use. Nevertheless, if a person with aphasia is assessed many years post-onset, it is possible that a lack of use of one of their languages is now compounding their language impairment resulting from the stroke (Goral, Naghibolhosseini, and Conner 2013). This is particularly true for lexical retrieval, a skill that is affected by aphasia and by language attrition. Due to the challenges in obtaining objective information about pre- and postmorbid proficiency, we might find a discrepancy between a person's self-report and the objectively measured language ability.

\subsection{Considering Culture and Identity}

It is important to assess - and potentially treat - all languages of multilingual speakers with aphasia not only to obtain an accurate picture of their spared abilities and main difficulties, but also out of respect to the person's identity. The languages multilingual people know and use, and the cultures associated with these languages, are an integral part of the identity of multilingual individuals. Multilingual people who are immigrants and who live in a sociolinguistic environment in which mostly their L2 (or a late-acquired language) is used, typically have access to services only in the language of the majority. But that language may not be the better spared language of a multilingual individual with aphasia, thereby obscuring their true language abilities. Logistically, assessing all languages of multilingual people who live in linguistic environments restricted to one or two of their languages may be challenging. For example, assessing our P1's Singhalese proved difficult due to lack of testing materials and available interpreters. At the same time, multilingual individuals may have good insight into their language abilities and may choose not to be assessed in a given language. For example, our P2 reported minimal use of Urdu for many years and indicated that it would not be useful assessing it, in contrast to his more relevant Farsi, which was assessed. 
If a participant is not interested in being assessed in a particular language, this should be followed out of respect to the person's wish and also because the lack of motivation can negatively affect performance. Such unnecessary additional anxiety associated with testing should be minimised (e.g. Knoph 2013). A related consideration is the person's ability to present themselves as they were, including presenting their multilingualism and multiculturalism. Many multilingual individuals take pride in their linguistic skills and wish to convey their abilities even if they can no longer display them. This is especially true when family members or other individuals from the person's community are present during the assessment. Eliciting information about this aspect of the history of people with aphasia is important for the integrity of services clinicians can provide.

\section{Multilingual Clinicians and Interpreters}

In the assessment of several languages, ideally, one would wish to use multilingual speech-language therapists (SLTs) to administer the testing, but multilingual clinicians with the same languages as the multilingual clients are often not available. In large countries, like the US, one would think that this is an achievable goal. Santhanam and Parveen (2018), however, report that only $6.5 \%$ of the members of the American Speech-LanguageHearing Association (ASHA) classify themselves as providers of bilingual services, and only $2 \%$ of the SLTs speak other languages than Spanish. In smaller communities, this proportion of multilingual clinicians is likely to be smaller.

Several countries (e.g. the US, UK, and Australia) have clinical guidelines which are developed by speech-language pathology and stroke organisations (Huang, Siyambalapitiya, and Cornwell 2019). These guidelines highlight the importance of SLTs collaborating with professional interpreters to ensure appropriate clinical service. The charge to provide appropriate and quality services without discrimination is, for instance, included in the professional codes of ethics in both the US and in Australia. As indicated in ASHA's Code of Ethics, SLTs who are members of the association are bound to provide linguistically and culturally appropriate services to their clients. This means that in service provision ASHA members must consider how language disorders might be identified and incorporate this knowledge into all areas of practice, including diagnosis and treatment. In an ideal world, this is a desired requirement. However, to what extent is it achievable? To be able to provide linguistically and culturally appropriate services, clinicians ideally should have some practice in working with multilingual speakers with language disorders, they should be trained in working with interpreters, and they should have access to appropriate assessment and treatment material in any language their clients may speak. Yet evidence suggests that this is not typically the case. Centeno $(2009,2015)$ has conducted two surveys in the US on service delivery to culturally and linguistically diverse 
populations with language disorders. According to his results, most of the respondents - SLTs working with multilingual adult speakers with language disorders - reported that they lacked such professional preparation in their education. Almost $80 \%$ of the respondents answered that they had no to minimal professional preparation to serve bilingual speakers. These results were corroborated in a recent Norwegian study, replicating Centeno's 2015 study (Norvik, Lind, and Jensen under revision), where as much as $96 \%$ of the recipients reported that they felt unequipped to work with multilingual speakers with aphasia.

\subsection{The Use of Interpreters}

How can we overcome the paucity of multilingual clinicians? Roger and Code (2011) found that the validity of a test can be maintained if professional interpreters use language tests that are designed or adapted for the language in question and the interpreters have been briefed about the assessment tool. It is important to bear in mind that the use of interpreters may influence the results of an assessment. In this regard, there are several possible challenges and aspects to consider. In a recent systematic review Huang and colleagues (2019) address several challenges regarding the use of interpreters. The greatest concern refers to an uncertainty of the accuracy of interpretations during assessment. This applies to whether the interpreter gives the correct instructions or items of an assessment tool, and also to whether the interpreter is able to convey the accurate responses of a person with aphasia. There is a great risk of missing important information about the forms and nuances of the language. It is easy to imagine that providing the correct information about speech errors resulting from apraxia of speech or dysarthria is hard for an interpreter. It is highly challenging to correctly interpret the speech and language production characteristic of aphasia, including pragmatic and prosodic deficits. All these concerns are crucial to obtain sufficient information from the assessment to plan appropriate therapy. In addition to these challenges, there are other, more logistical challenges, such as difficulties in accessing professional interpreters of all the needed languages.

When an appropriate interpreter is recruited there are still pitfalls. To avoid some of these, the interpreter needs information about aphasia and instructions on how to facilitate good communication with individuals with aphasia, for example, to give sufficient response time in the assessment situation; this is in line with the suggestions from ASHA (2004). It is not unusual for interpreters to inadvertently change test stimuli or fail to convey the client's exact responses (Roberts 2008). Since the SLT does not speak the language under consideration, the above may happen without his/her awareness. To avoid this, the interpreter should be instructed by the clinician on how to conduct a valid assessment; i.e. not to change or elaborate on the stimuli, not to construe what the person with aphasia is saying but 
to convey the exact words and meanings, with their flaws, and not to try to help the person with aphasia in finding the right word, for instance.

For assessment of the different languages of multilingual speakers with aphasia, it is often recommended to adhere to a so-called monolingual mode; that is, to stick to only the target language of the assessment. In a monolingual setting, the language not being used is thought to be less active (e.g. Grosjean 2013; but cf. Guo et al. 2011), and this may assist the person being tested to avoid mixing the two languages. With our two participants we aimed to achieve this, but working with most interpreters living in Norway, this is not possible. The person being tested, for instance, in his L1 knows that the interpreter knows Norwegian, and most probably also English, in addition to the target language of the assessment. Additionally, even if the interpreters are instructed on how to conduct the assessment, they will usually communicate some with the clinician who is in the room; hence, there will be some input from languages other than the target language. The same may happen when the family members of the person with aphasia are in the room. In addition, the person with aphasia will often seek help from the family member when encountering difficult tasks or items, which will influence their performance.

Another issue may be that, in smaller communities, there is a shortage of interpreters who know certain languages, and one might need to use the same interpreter for two different languages (e.g. Urdu and Punjabi). Other challenges linked to smaller language communities may be that the person with aphasia knows the interpreter from before and might therefore not accept the interpreter. In some cultures, having a language problem like aphasia is shameful, and the person or the family may not want to get help, for fear of being judged by the community. In the cases where there are no available interpreters, one can train a grown-up family member to assist in the assessment. However, professional interpreters enhance objectivity and will most likely convey complex and specific information in a more accurate manner (McLeod and Verdon 2017).

\section{Linguistically Appropriate Assessment Tools}

The great diversity in multilingual speakers with aphasia poses challenges in terms of assessment tools. If the goal is to assess all languages of a multilingual speaker, a fundamental need is appropriate assessment tools in all the languages. Most tools are developed for monolingual people and may thus not be applicable to multilingual speakers.

First, the procedure of formal testing in itself is culturally dependent. Using two-dimensional pictures and restricting feedback from the examiner may be less common in some cultures. The mere idea of telling a story based on a two-dimensional set of drawings may be completely foreign and thus awkward in some cultures, such as in Sri Lanka (as indicated by the interpreter who worked with P1). 
In addition, a translation of a tool is not sufficient. Stimuli may be culturally and/or linguistically inappropriate and linguistic constructions may have different levels of difficulty in the translated version. For instance, when we used an action-picture test to assess verb production, we needed to consider culturally inappropriate drawings, such as a woman showering, given the cultural background of our participants, or actions that are atypical (e.g. shovelling snow). We also had to exclude, or score separately, actions that are referred to with two or more words in certain languages (e.g. mopping or shaving in Urdu; peeling or combing in Farsi, which are expressed as noun-verb compounds). Similarly, the BAT sentence comprehension subtest includes passive constructions, but these may be very uncommon in languages such as Farsi.

Further, some linguistic constructions may be non-existent in a given language. To be able to compare the person's performance in their two (or more) languages, the assessment tools must be comparable on the item and subtest level. Even for tests that have been developed in a variety of languages with careful linguistic and cultural considerations, there are no norms available from multilingual individuals. For example, the BAT, which is designed with bilingualism in mind, still does not have sufficient data on the degree to which different language versions are comparable in difficulty. Therefore, comparing performance across languages within an individual should be done with caution; for instance, both P1 and P2 achieved somewhat different overall scores in the BAT across their languages (e.g. P1's Norwegian BAT score was more than $10 \%$ higher than the other two languages). However, because there is no evidence that the BAT Norwegian, Tamil, and Farsi versions are comparable, we could not be sure that the participant's performance in Norwegian is actually better. P1's scores on the object-naming test seem to corroborate this conclusion (see Table 9.1), although, here too, we cannot be sure that the tests are comparable across the languages. This highlights the importance of using several measures in testing the same individual to obtain a more complete picture of their relative language abilities.

As mentioned above, in several survey studies among SLTs in the US and in Norway, nearly all SLTs report that they lack linguistically and culturally appropriate assessment material (Centeno 2015; Norvik, Lind, and Jensen under revision). To overcome some of the challenges associated with suitable assessment material in all the languages of a multilingual speaker, many of the survey respondents reported collecting connected-language production in the speaker's two languages, in addition to gathering information about the two languages from the family or from relatives.

Indeed, in addition to standardised testing, the elicitation and examination of narrative production in each of the languages of multilingual speakers with aphasia is an approach that can provide information about the strength and weaknesses in all the languages of the individual with aphasia. In such less-formal assessments, connected-language production is elicited 


\section{2}

from individuals with aphasia, using picture scenes, picture sequences, or narrative topics, in each of their languages. As a rule, little adaptation is required with such elicitation material, although, here too, picture stimuli and topics should be culturally appropriate. Once the samples are collected, a variety of micro- and macro-level analyses can be done to assess aspects like word-finding difficulties, sentence structure, coherence, and code-switching, amongst others (e.g. Boyle 2014, 2015; Conner et al. 2018; Knoph, Simonsen, and Lind 2017). We address challenges of analysis and interpretation of informal assessment of language production next.

\section{Interpreting the Results}

The interpretation of the results obtained from multilingual assessment can be challenging due to two main factors: the contribution of (pre-stroke) language proficiency to the observed performance and scoring mixed language production.

Information about language proficiency is essential for accurate interpretation of aphasia testing in multilingual speakers. Any decreased performance on an assessment test administered with multilingual individuals with aphasia must be interpreted in the context of the individuals' estimated preonset abilities, to make sure an error is attributed correctly to the acquired deficit rather than to a partial mastery of the target language. However, teasing apart aphasia-related and non-native proficiency-related difficulties is often challenging. Indeed, as discussed above, one of the fundamental challenges in assessing language and communication abilities of multilingual individuals with aphasia is the inherent uncertainty regarding their language and communication abilities prior to the aphasia onset. This is typically not the case when assessing monolingual individuals with aphasia. For monolingual speakers who are assessed in their native-language environment, the implicit assumption is that they had a complete mastery of all aspects of their native language prior to the aphasia onset, if no previous language or reading/writing disorder is known. Exceptions here are literacy skills, which vary across individuals, and perhaps vocabulary size and metalinguistic abilities, which typically increase with education levels. In contrast, when assessing multilingual individuals with aphasia, proficiency levels in each language may range greatly and thus a similar assumption regarding proficiency cannot be made. And yet no, or limited, objective data about pre-stroke abilities are typically available. Short of the occasional videorecording or audio-recording of an event or an interview that the person with aphasia may have had from before their stroke, SLTs need to rely on self-report when attempting to establish levels of proficiency in the various languages of a multilingual, across modalities and in various contexts, and these are problematic as discussed above.

Another great challenge when interpreting the assessment of multilingual individuals with aphasia concerns language mixing. Language 
mixing, typical of multilingual conversations, includes inserting elements from more than one language in an utterance (termed code-mixing), and changing a language between utterances or between communication turns (termed code-switching). When multilingual speakers communicate with interlocutors who share the same languages, language mixing is typical, although speakers and cultures vary in the degree and frequency with which they mix their languages (e.g. Bullock and Toribio 2012). When communicating with interlocutors who share only one of their languages, neurologically healthy multilinguals are highly adept at refraining from inappropriate language mixing. Moreover, language mixing is highly sensitive to contextual cues, such as the topic of conversation and the identity of the interlocutors.

It has been suggested that multilingual people with aphasia may mix their languages with greater frequency than neurologically healthy speakers, at least during testing situations that require the use of one language (e.g. Muñoz, Marquardt, and Copeland 1999; Paplikar et al. 2016). However, frequent language mixing has been found especially when the people with aphasia know that their interlocutors share their languages, pointing to a possible strategic use of mixing rather than a failure to control the language of production (Goral, Norvik, and Jensen 2019). Therefore, when multilingual people with aphasia are tested in each of their languages, most often by an interlocutor who is also multilingual, it is difficult to assure a monolingual mode of the communication situation and thus to minimise language mixing.

When multilingual individuals with aphasia produce language mixing during testing, decisions must be made regarding how to score correct responses in the non-target language and how to score mixed utterances. Moreover, for languages that share a large number of cognates, it is sometimes difficult to determine if a word was produced in the non-target language or was simply produced with a non-target accent (for example, milk produced for melk during Norwegian testing of an English-Norwegian speaker). As well, a non-target word may be produced because it is a semicognate or a false friend in the patient's two languages (for example, coppa produced by a Spanish-Italian speaker for the word glass during Italian testing).

In connected-language production, multilingual individuals with aphasia are likely to score higher on a variety of communicative measures if their output in either language is combined and considered correct, as compared to when only what they produced in the target language is scored as correct (Lerman, Goral, and Obler 2020). This is similar to what has been found with neurologically healthy multilingual speakers who have varying levels of proficiency in their two languages (e.g. Gollan et al. 2007; Kohnert, Hernandez, and Bates 1998). Therefore, the scoring and interpretation of an assessment conducted with multilingual individuals with aphasia should be completed by individuals who are proficient in the same languages. 


\section{Monica Norvik and Mira Goral}

\section{Conclusions and Future Directions}

We reviewed here the main challenges associated with the assessment of linguistic and communication skills in multilingual individuals who acquire aphasia. On the one hand, it is imperative to assess all languages of multilingual individuals, to gain insight into their levels of spared and impaired language abilities. On the other hand, due to the limited availability of assessment tools that are appropriately adapted to a variety of languages and cultures, and with virtually no multilingual norms that reflect the heterogeneity found among multilingual individuals, aphasia batteries and tests should be used with caution while avoiding direct comparisons of performance on different tests in different languages. The BAT, while a useful tool that is freely available in multiple languages, requires several modifications and additional normative data.

Due to variability in performance of individuals with aphasia, repeated testing and establishing performance stability has been recommended (Thompson 2014). Repeated testing of multilingual individuals introduces multiple repetitions of the same content, unless comparable lists are developed (Borodkin, Goral, and Kempler 2020). The development of appropriate assessment tests for use with multilingual users is clearly needed. Furthermore, to alleviate some of these challenges, we advocate for the implementation of less formal assessments, using elicited connected-language production, to the extent possible. Here, scoring and interpretation challenges may be addressed with the collection of detailed language background, proficiency and use history, as well as by a careful analysis of errors and their likely sources, language mixing patterns, and communication success in each language as well as in all languages, potentially combining psycholinguistic and sociolinguistic analyses.

Further training of SLTs to work with multilingual individuals and increasing SLTs' sensitivity to cultural and linguistic differences, would make the assessment process more efficient and more accurate. As well, thorough training of interpreters and improving collaboration between them and SLTs would facilitate more accurate assessment. Additional future directions that could be explored include the application of tools, such as event-related potentials (ERPs) and eye trackers, to examine online processing of linguistic information, although these tools may not be as useful in clinical settings as in the research context. The collection of such data may reduce the need to collect and score overt responses to structured tests and may increase the efficiency of the assessment of language.

\section{References}

American Speech-Language-Hearing Association. 2004. Knowledge and Skills Needed by Speech-Language Pathologists and Audiologists to Provide Culturally and Linguistically Appropriate Services [Knowledge and Skills]. 
www.asha.org/policy. Index terms: multicultural issues. doi:10.1044/policy. KS2004-00215

Ansaldo, A.I., K. Marcotte, L. Scherer, and G. Raboyeau. 2008. "Language Therapy and Bilingual Aphasia: Clinical Implications of Psycholinguistic and Neuroimaging Research." Journal of Neurolinguistics 21 (6): 539-557. doi:10.1016/j.jneuroling.2008.02.001

Bak, T.H. and J.R. Hodges. 2003. "Kissing and Dancing - A Test to Distinguish the Lexical and Conceptual Contributions to Noun/Verb and Action/ Object Dissociation. Preliminary Results in Patients with Frontotemporal Dementia." Journal of Neurolinguistics 16 (2): 169-181. doi:10.1016/ S0911-6044(02)00011-8

Boyle, M. 2014. "Test-retest Stability of Word Retrieval in Aphasic Discourse." Journal of Speech, Language, and Hearing Research 57 (3): 966-978. doi:10.1044/ 2014_JSLHR-L-13-0171

Boyle, M. 2015. "Stability of Word-Retrieval Errors with the AphasiaBank Stimuli." American Journal of Speech-Language Pathology 24 (4): 953-960. doi:10.1044/2015_AJSLP-14-0152

Borodkin, K., M. Goral, and D. Kempler. 2020. "Measuring Performance Stability in Persons with Aphasia: Identical versus Comparable Testing Forms.” Aphasiology 34 (3): 376-390. doi:10.1080/02687038.2019.1670331

Bullock, B. and A.J. Toribio, eds. 2012. The Handbook of Linguistic CodeSwitching. Cambridge: Cambridge University Press.

Centeno, J.G. 2009. "Serving Bilingual Patients with Aphasia: Challenges, Foundations, and Procedures." Revista de Logopedia, Foniatria, y Audiología 29 (1): 30-36. doi:10.1016/S0214-4603(09)70141-X

Centeno, J.G. 2015. "Assessing Services with Communicatively Impaired Bilingual Adults in Culturally and Linguistically Diverse Neurorehabilitation Programs." Journal of Communication Disorders 58: 58-73. doi:10.1016/j. jcomdis.2015.10.005

Centeno, J.G. and A.I. Ansaldo. 2013. "Aphasia in Multilingual Populations." In Aphasia and Related Neurogenic Communication Disorders, edited by I. Papathanasiou, P. Coppens, and C. Potagas, 275-294. Burlington, MA: Jones \& Barlett Learning.

Conner, P.S., M. Goral, I. Anema, K. Borodkin, Y. Haendler, M. Knoph, C. Mustelier, E. Paluska, Y. Melnikova, and M. Moeyaert. 2018. "The Role of Language Proficiency and Linguistic Distance in Cross-Linguistic Treatment Effects in Aphasia." Clinical Linguistics \& Phonetics 32 (8): 739-757. doi:10.10 80/02699206.2018.1435723

Edmonds, L.A. and S. Kiran. 2006. "Effect of Semantic Naming Treatment on Crosslinguistic Generalization in Bilingual Aphasia." Journal of Speech, Language, and Hearing Research 49 (4): 729-748. doi:10.1044/1092-4388(2006/053)

Fabbro, F. 2001. "The Bilingual Brain: Bilingual Aphasia." Brain and Language 79 (2): 201-210. doi:10.1006/brln.2001.2481

Flowers, H.L., F.L. Silver, J. Fang, E. Rochon, and R. Martino. 2013. "The Incidence, Co-Occurrence, and Predictors of Dysphagia, Dysarthria, and Aphasia After First-Ever Acute Ischemic Stroke." Journal of Communication Disorders 46 (3): 238-248. doi:10.1016/j.jcomdis.2013.04.001

Fyndanis, V., M. Lind, S. Varlokosta, M. Kambanaros, E. Soroli, K. Ceder, K.K. Grohmann et al. 2017. "Cross-linguistic Adaptations of the Comprehensive 


\section{Monica Norvik and Mira Goral}

Aphasia Test: Challenges and Solutions." Clinical Linguistics and Phonetics 31 (7-9): 697-710. doi:10.1080/02699206.2017.1310299

Gollan, T.H., C. Fennema-Notestine, R.I. Montoya, and T.L. Jernigan. 2007. "The Bilingual Effect on Boston Naming Test Performance." The Journal of the International Neuropsychological Society 13 (2): 197-208. doi:10.1017/ S1355617707070038

Goodglass, K., E. Kaplan, and B. Barresi. 2000. Boston Diagnostic Aphasia Examination, 3rd edition. San Antonio, TX: The Psychological.

Goral, M. 2015. "Bilingual Aphasia.” In Routledge Handbook of Communication Disorders, edited by R. Bahr and E. Silliman, 367-378. New York: Routledge.

Goral, M., M. Naghibolhosseini, and P.S. Conner. 2013. "Asymmetric Inhibitory Treatment Effects in Multilingual Aphasia.” Cognitive Neuropsychology 30 (78): 564-577. doi:10.1080/02643294.2013.878692

Goral, M., M.I. Norvik, and B.U. Jensen. 2019. "Variation in Language Mixing in Multilingual Aphasia." Clinical Linguistics \& Phonetics 33 (10-11): 915-929. doi:10.1080/02699206.2019.1584646

Graetz, P., R. De Bleser, and K. Willmes. 1992. Akense Afasie Test (AAT) [The Aken Afasia Test, ATT]. Lisse, Switzerland.

Grosjean, F. 2013. "Bilingualism: A Short Introduction.” In The Psycholinguistics of Bilingualism, edited by F. Grosjean and P. Li, 5-26. Malden, MA: Wiley-Blackwell.

Guo, T., H. Liu, M. Misra, and J.F. Kroll. 2011. "Local and Global Inhibition in Bilingual Word Production: fMRI Evidence from Chinese-English Bilinguals." Neuroimage 56 (4): 2300-2309. doi:10.1016/j.neuroimage.2011.03.049

Howard, D. and K. Patterson. 1992. The Pyramids and Palm Trees Test. Bury St. Edmunds, UK: Thames Valley Test Company.

Huang, A.J.R., S. Siyambalapitiya, and P. Cornwell. 2019. "Speech Pathologists and Professional Interpreters Managing Culturally and Linguistically Diverse Adults with Communication Disorders: A systematic review." International Journal of Language \& Communication Disorders 5(5): 689-704. doi:10.1111/1460-6984.12475

Kaplan, E., H. Goodglass, and S. Weintraub. 2001. Boston Naming Test, 2nd edition. Austin, TX: Pro-Ed.

Kay, J., R. Lesser, and M. Coltheart. 1992. PALPA: Psycholinguistic Assessments of Language Processing in Aphasia. London, UK: Erlbaum.

Kertesz, A. 2006. Western Aphasia Battery Test Manual. San Antonio, TX: The Psychological Corporation.

Knoph, M.I.K. 2013. "Language Intervention in Arabic-English Bilingual Aphasia: A Case Study.” Aphasiology 27 (12): 1440-1458. doi:10.1080/02687038.2013.8 32139

Knoph, M.I.N., H.G. Simonsen, and M. Lind. 2017. "Cross-linguistic Transfer Effects of Verb-Production Therapy in Two Cases of Multilingual Aphasia." Aphasiology 31 (12): 1482-1509. doi:10.1080/02687038.2017.1358447

Kohnert, K.J., A.E. Hernandez, and E. Bates. 1998. "Bilingual Performance on the Boston Naming Test: Preliminary Norms in Spanish and English." Brain and Language 65 (3): 422-440. doi:10.1006/brln.1998.2001

Kuvač Kraljević, J., A. Matić, and K. Lice. 2020. "Putting the CAT-HR Out: Key Properties and Specificities.” Aphasiology 34 (7): 820-839. doi:10.1080/02687 038.2019.1650160 
Kuzmina, E., M. Goral, M.I. Norvik, and B.S. Weekes. 2019. "What Influences Language Impairment in Bilingual Aphasia? A Meta-Analytic Review.” Frontiers in Psychology 10: 445. doi:10.3389/fpsyg.2019.00445

Lapointe, L.L. and J. Horner. 1998. Reading Comprehension Battery for Aphasia-2. Austin, TX: Pro-Ed.

Lerman, A., M. Goral, and L. K. Obler. 2020. “The Complex Relationship between Pre-Stroke and Post-Stroke Language Abilities in Multilingual Individuals with Aphasia.” Aphasiology 34 (11): 1319-1340. doi:10.1080/02687038.2019.167 3303

Lomas, J., L. Pickard, S. Bester, H. Elbard, A. Finlayson, and C. Zoghaib. 1989. "The Communicative Effectiveness Index. Development and Psychometric Evaluation of a Functional Communicative Measure for Adult Aphasia." Journal of Speech and Hearing Disorders 54 (1): 113-124. doi:10.1044/jshd. 5401.113

Marian, V., H.K. Blumenfeld, and M. Kaushanskaya. 2007. “The Language Experience and Proficiency Questionnaire (LEAP-Q): Assessing Language Profiles in Bilinguals and Multilinguals." Journal of Speech Language Hearing Research 50 (4): 940-967. doi:10.1044/1092-4388(2007/067)

McLeod, S. and S. Verdon. 2017. “Tutorial: Speech Assessment for Multilingual Children Who Do Not Speak the Same Language(s) as the Speech-Language Pathologist." American Journal of Speech-Language Pathology 26 (3): 691-708. doi:10.1044/2017_AJSLP-15-0161

Muñoz, M.L., T.P. Marquardt, and G. Copeland. 1999. “A Comparison of the Codeswitching Patterns of Aphasic and Neurologically Normal Bilingual Speakers of English and Spanish." Brain and Language 66 (2): 249-274. doi:10.1006/ brln.1998.2021

Murray, L.L. and P. Coppens. 2017. "Formal and Informal Assessment of Aphasia." In Aphasia and Related Neurogenic Communication Disorders, 2nd edition, edited by I. Papathanasiou and P. Coppens, 81-108. Burlington, MA: Jones \& Barlett Learning.

Norvik, M.I., M. Lind, and B.U. Jensen. Under revision. "Working with Multilingual Aphasia: Attitudes and Practices Among Speech and Language Therapists in Norway." International Multilingual Research Journal.

Papathanasiou, I., Coppens, P., and Davidson, B. 2017. "Aphasia and Related Neurogenic Communication Disorders: Basic Concepts, Management, and Efficacy." In Aphasia and Related Neurogenic Communication Disorders, 2nd edition, edited by I. Papathanasiou and P. Coppens, 3-14. Burlington, MA: Jones \& Barlett Learning.

Paplikar, A., M. Goral, M. Gitterman, and L. K. Obler. 2016. "Directionality of Language-Mixing in Bilinguals with Non-Fluent Aphasia." Poster presented at the Academy of Aphasia, Wales.

Paradis, M. 2004. A Neurolinguistic Theory of Bilingualism. Philadelphia, PA: John Benjamins Publishing Company.

Paradis, M. and G. Libben. 1987. The Assessment of Bilingual Aphasia. Hillsdale, NJ: Lawrence Erlbaum Associates.

Rautakoski, P., A-M. Korpijaakko-Huuhka, and A. Klippi. 2008. "People with Severe and Moderate Aphasia and Their Partners as Estimators of Communication Skills: A Client-cantered Evaluation." Aphasiology 22 (12): 1269-1293. doi:10.10 80/02687030802374788 


\section{Monica Norvik and Mira Goral}

Roberts, P.M. 2008. "Aphasia Assessment and Treatment for Bilingual and Culturally Diverse Patients." In Language Intervention Strategies in Aphasia and Related Neurogenic Communication Disorders, 5th edition, edited by R. Chapey, 208-232. Baltimore, MA: Lippincott Williams \& Wilkins.

Roberts, P.M. and S. Kiran. 2007. "Assessment and Treatment of Bilingual Aphasia and Bilingual Anomia." In Speech and Language Disorders in Bilinguals, edited by A. Ardila and E. Ramos, 109-130. New York, NY: Nova Science Publishers, Inc.

Roger, P. and C. Code. 2011. "Lost in Translation? Issues of Content Validity in Interpreter-mediated Aphasia Assessments." International Journal of Speech and Language Pathology 13 (1): 61-73. doi:10.3109/17549507.2011.549241

Santhanam, S.P. and S. Parveen. 2018. "Serving Culturally and Linguistically Diverse Clients: A Review of Changing Trends in Speech-Language Pathologists' Selfefficacy and Implications for Stakeholders." Clinical Archives of Communication Disorders 3 (3): 165-177. doi:10.21849/cacd.2018.00395

Schmid, M.S. 2013. "First Language Attrition." Linguistic Approaches to Bilingualism 3 (1): 94-115. doi:10.1075/lab.3.1.05sch

Swinburn K, G. Porter, and D. Howard. 2004. Comprehensive Aphasia Test. Hove, UK: Psychology Press.

Thompson, C.K. 2012. Northwestern Assessment of Verbs and Sentences (NAVS). Evanston, IL: Northwestern University.

Thompson, C.K. 2014. "Establishing the Effects of Treatment for Aphasia Using Single-Subject-Controlled Experimental Designs.” Aphasiology 15 (5): 588-597. doi:10.1080/02687038.2014.987043

Tomoschuk, B., V. Ferreira, and T. Gollan. 2019. "When a Seven is Not a Seven: Self-Ratings of Bilingual Language Proficiency Differ Between and within Language Populations.” Bilingualism: Language and Cognition 22 (3): 516-536. doi:10.1017/S1366728918000421 


\title{
10 Pathological Language- Switching/Mixing and Its Relationship to Domain-General Cognitive Control
}

\author{
Valantis Fyndanis and Minna Lebtonen
}

\section{Introduction}

Language-switching (or, alternatively, code-switching) and language-mixing are typical and common bilingual behaviours that have been the focus of interest both in sociolinguistic (e.g. Lanza [1997] 2004) and psycholinguistic research on bilingualism (e.g. Myers-Scotton [1993] 1997). Codeswitching and -mixing behaviour can be influenced by social and individual factors, such as the topic, the interlocutors, or an individual's language mode (e.g. Grosjean 1985). Switching and mixing can also be deliberately used to achieve various communicative goals (Grosjean 1985). The terms language-mixing and language-switching are not interchangeable. According to Paradis (1977) and Albert and Obler (1979), mixing refers to intermingling two or more languages within the same utterance. Switching, instead, refers to the alternation between different languages across utterances, not within utterances. Most scholars have adopted the definition above (e.g. Fabbro 1999b; Fabbro, Skrap, and Aglioti 2000; Leemann et al. 2007). However, some scholars use the term language-mixing to refer to both code-mixing and code-switching (e.g. Lerman et al. 2019), while the term language-switching is typically used in psycholinguistic experiments, including single-word tasks (e.g. Meuter and Allport 1999; Declerck and Philipp 2015). In the present chapter, we distinguish between language-switching and language-mixing, adopting the most widely accepted definition (e.g. Paradis 1977; Albert and Obler 1979). Nevertheless, we will use the term language-switching when referring to single-word studies, as the majority of such studies do so.

There is a great deal of work in the psycholinguistics of language-switching and mixing that has focused on bilingual language acquisition and the extent to which the two languages are differentiated in early childhood (e.g. Genesee 1989; Lanza 1992). Recently, there has been a lot of interest in the possible advantages of lifelong bilingualism in cognitive control (executive functions, EFs), and studies have investigated various aspects of bilingual experience that might be related to cognitive control (e.g. Luk, De Sa, and Bialystok, 2011; Soveri et al. 2011). One such aspect is language-switching 
or mixing, which, in addition to requiring retrieval of lexical and morphosyntactic elements in the target language, is assumed to pose demands on cognitive control. Overall, well-functioning EFs such as inhibitory control, set-shifting, and monitoring are believed to be central to smooth bilingual language use, including staying in one language and switching to another when appropriate (e.g. Green 1998). In addition, one needs to pay attention to and monitor one's language use for possible errors and inaccuracies, including whether the language used is the correct one in the communicative situation. Since such domain-general EFs (i.e. EFs not restricted to language) are assumed to be active in language-switching, lifelong bilingual experience with frequent switching has been hypothesised to train these functions, leading also to a bilingual advantage in nonverbal EF tasks (e.g. Bialystok et al. 2009). Hence, research has focused on the possible enhancing role of language-switching or mixing behaviour in one's general cognitive control abilities.

Empirical evidence for the role of cognitive control in language-switching comes primarily from so-called asymmetric switching costs observed in language-switching tasks performed in the laboratory. In such experiments, participants typically name pictures in one language, and naming times are measured for trials in which the language of the naming needs to be switched and compared to trials where the same language is repeatedly used. Switch trials tend to elicit a processing cost, and this cost has also been shown to be asymmetric: switches from L2 to L1 take more time than switches from L1 to L2 (e.g. Meuter and Allport 1999). This unintuitive asymmetry has been suggested to reflect suppression of a stronger L1 during production of a weaker L2. Resolving this inhibition when switching back to L1 is assumed to be cognitively costly. The common assumption is that such control processes involved in language processing are largely shared with domain-general cognitive control (e.g. Abutalebi and Green 2007). For example, suppression of the other language engages domain-general inhibitory processes also involved in other (nonlinguistic/nonverbal) tasks. This assumption, however, deserves further scrutiny.

The central assumption behind the assumed bilingual training effects is that, since the domain-general EFs engaged by language-switching are also used in nonlinguistic/nonverbal tasks, language-switching can train also processes important in nonlinguistic/nonverbal EFs. While such training effects in bilingualism have been recently strongly debated (e.g. Paap et al. 2015; Bialystok 2017) and questioned by meta-analyses (see, for example, de Bruin et al. 2015; Lehtonen et al. 2018), it is still an open question to what extent the engagement hypothesis holds; in other words, to what extent domain-general EFs are involved in bilingual language use. An alternative hypothesis is that the cognitive system develops specialised solutions for different subfunctions while learning to perform a task, and that language control would be specific and distinct from nonlinguistic/nonverbal cognitive control (Jylkkä 2017). 
An interesting perspective on the relationship between language-switching or mixing and cognitive control emerges from studies on bilingual persons with aphasia ${ }^{1}$ (PWA) who exhibit behaviour that appears as uncontrolled language-switching or mixing. A number of such cases have been reported, but whether they truly reflect a control deficit or perhaps a communicative strategy to compensate for deficient abilities in one language remains an unresolved issue (see e.g. Goral, Norvik, and Jensen 2019). This question also touches upon the relationship and possible overlap between language control and domain-general cognitive control: if a language control deficit seems to underlie the switching/mixing behaviour of such participants, is this impairment also accompanied by a nonverbal control deficit? The present review focuses on these issues.

\subsection{Definitions of Pathological and Non-Pathological Language-Mixing and Switching}

Both pathological language-mixing and pathological language-switching refer to the alternation between different languages without control by a given speaker (Fabbro, Skrap, and Aglioti 2000). It is assumed that pathological mixing and switching are exhibited by speakers with deficient cognitive control (e.g. Abutalebi, Miozzo, and Cappa 2000; Ansaldo, Saidi, and Ruiz 2010; Fabbro 1999a). When this control fails and the speaker's language selection does not adhere to the language of the interlocutors and context, mixing and switching may result in communication breakdown. Communication breakdown occurs when the interlocutor does not understand one (or more) of the languages that the multilingual speaker uses within or across utterances, and so s/he fails to comprehend the speech of the person who mixes or switches between languages.

Not all researchers, however, agree on the usefulness of the term pathological language-switching or mixing. Goral, Norvik, and Jensen (2019), for example, pointed out that not only PWA but also healthy speakers exhibit mixing/switching behaviours, with the differences between neurologically healthy speakers' and PWA's mixing/switching behaviours being quantitative rather than qualitative - PWA tend to mix/switch to a greater extent compared to neurologically healthy speakers (e.g. Bhat and Chengappa 2005; Gardner-Chloros 2009; Paplikar 2016). Thus, also based on their results, Goral, Norvik, and Jensen (2019) joined Grosjean (1985) and argued against the use of the terms pathological mixing and pathological switching in future studies on multilingual aphasia, suggesting that multilingual PWA adopt a cooperative, not competitive, language activation schema (Green and Wei 2014), and use language-mixing/switching as a communicative strategy. As per Goral, Norvik, and Jensen (2019), therefore, multilingual PWA's languages do not compete for selection, but complement each other, so that PWA can draw on all of their linguistic resources (i.e. on all of their languages) in order to communicate. Language-switching/mixing could, thus, be seen as 


\section{2}

\section{Valantis Fyndanis and Minna Lehtonen}

a result of this communicative strategy. Goral, Norvik, and Jensen (2019) attributed the switching/mixing behaviour of the speakers who participated in their study to word-retrieval problems. One could argue, however, that it is not a functional communicative strategy to switch to a language that the interlocutor does not understand. Instead, when encountering word-retrieval problems, multilingual speakers would rather resort to circumlocutions adhering to the shared language(s), if they were able to control the language of the utterances. In other words, it is central to consider whether languageswitching or mixing is taking place with an examiner that the multilingual PWA knows or assumes to be multilingual who understands all of his/her languages. In such cases, mixing/switching between languages would not lead to communication breakdown, even if mixing/switching is not encouraged in the situation. Therefore, it could be seen as a communicative strategy of the participant, and not as an inability to control one's languages.

We suggest that the two opposing views above could be reconciled by using the presence of communication breakdown as a diagnostic of pathological switching/mixing. In case the participant tends to switch to another language to convey a message, and if that language is shared between the interlocutors, this switching behaviour can be considered to be purposeful and, therefore, non-pathological. The same may apply to a controlled testing situation where the participant decides to say something rather than keep quiet, even if the utterance is in the incorrect/non-target language. Again, this kind of switching can be seen as an intentional communicative strategy. In contrast, participants' frequent switching/mixing in inappropriate situations (i.e. with monolingual interlocutors), which leads to communication breakdown, appears to be beyond the control and communicative intentions of the speaker. We argue that such switching behaviour can be considered pathological switching. Furthermore, we suggest that this holds regardless of whether the speakers who frequently switch or mix languages in inappropriate situations know that they should not switch/mix languages given the situation. The presence or lack of such awareness may reflect well-functioning or deficient-monitoring abilities, respectively, but monitoring abilities and inhibitory control, for example, are assumedly separable functions and, thus, can be impaired independently. Moreover, impairment in either function could lead to pathological switching/mixing. In the present review, we aim to scrutinise the reported research of pathological switching/mixing from this perspective, addressing whether the communicative strategy explanation advocated by Goral, Norvik, and Jensen (2019) applies to all or most cases of "pathological" switching/mixing considered in the literature.

\subsection{Does Language-Switching Share Aspects of General Task Switching?}

Research on pathological switching can shed light on cognitive control processes in "healthy" language-switching. Despite the common view 
that language-switching engages domain-general EFs, including inhibitory control, studies investigating the engagement hypothesis have not always produced clear-cut results that would support it. In fact, there are behavioural studies showing no clear overlap between one's language-switching efficiency and EF abilities, i.e. questioning the view that language-switching abilities would be (partly) dependent on one's general inhibition and shifting abilities (e.g. Jylkkä et al. 2018a, 2018b). Furthermore, bilingual language control and nonverbal cognitive control seem to show differential effects in ageing (e.g. Weissberger et al. 2012), suggesting these processes are not entirely shared.

At the level of the brain, one can ask whether the same areas are active for language-switching and domain-general task switching. The earliest studies associated left parietal and frontal areas with a language-switching mechanism (see e.g. Fabbro 1999a, 1999b). More recently, brain-imaging studies on healthy bilinguals have found language-switching to activate a number of different brain regions, those related to core aspects of language processing, such as the posterior temporal cortex, parietal areas, and the left inferior frontal cortex (Broca's area), as well as those related to cognitive control, such as the dorsolateral prefrontal cortex, anterior cingulate, and basal ganglia (see e.g. Moritz-Gasser and Duffau 2009, for a review). A meta-analysis that focused particularly on the regions involved in cognitive control during language-switching showed that the left frontal areas, bilateral temporal areas, as well as basal ganglia regions (e.g. the caudate nuclei) were most reliably activated by these aspects of switching (Luk et al. 2012). This network of areas has also been shown to be active in nonverbal switching (De Baene et al. 2015) and other domain-general EF tasks, providing evidence for overlap between language control and domain-general EFs. However, many separable functions can rely on the same coarse neuroanatomical regions, so this evidence can only be suggestive for that view.

Only a few studies on aphasia have thus far directly explored the relationship between language control and domain-general control. The results from studies by Dash and Kar (2014) and Gray and Kiran (2016) suggest that there is a dissociation between the two, lending support to a model of domain-specific cognitive control. Green et al. (2010) found a double dissociation (in two PWA) between performance in a nonverbal control task (Flanker) and performance in a linguistic control task (Stroop). The authors attributed this to different lesion sites of the participants: basal ganglia with the language control deficit and parietal cortex with the nonverbal control deficit. Verreyt et al. (2013), in turn, found evidence for a general control deficit underlying a bilingual PWA's greater impairments in one of the two languages, supporting the domaingeneral control view. As the findings are variable and based on limited samples, more studies from different perspectives are needed to settle the controversy. 


\section{Valantis Fyndanis and Minna Lebtonen}

\subsection{Aims of the Review}

The present review summarises research on the reported "pathological" switching in brain-damaged individuals, particularly PWA. First, we evaluate whether the reported switching/mixing patterns can be interpreted as reflecting a communicative strategy used to compensate for deficient linguistic resources, or an impaired ability to control language use. Second, we focus on cases that demonstrate truly pathological (uncontrolled) switching/ mixing and on these participants' performance in EF measures. If domaingeneral EFs are critically engaged in language-mixing/switching, individuals exhibiting pathological switching/mixing should also show deficits in nonverbal EF tasks, particularly in EF components assumed to be crucial for language-switching: inhibition, set-shifting, and monitoring. Third, we will make a note about participants' lesion sites and what we know about the role of different brain regions in healthy bilingual participants. A better understanding of the nature of language selection problems and their neural underpinnings in brain-damaged individuals can shed light on the role of cognitive control in bilingual language use and, thus, the relationship between language control and domain-general cognitive control.

\section{Review of Original Studies on Pathological Mixing/Switching ${ }^{2}$}

In this narrative review, we focus on studies addressing the issue of pathological switching/mixing or switching/mixing as a communicative strategy in participants with brain damage that in most cases resulted in aphasia. ${ }^{3}$ Our focus is particularly on three aspects of the studies of so-called pathological mixing and switching: 1) whether language-mixing and/or switching is observed in genuine monolingual situations (that is, in situations where the participant with brain damage knows that the examiner can understand only one of their languages) where switching can be assumed to lead to communication breakdown and, thus, constitutes "true" pathological mixing/ switching; 2) participants' performance on cognitive tasks, particularly in EF tasks; and 3) lesion site in the brain. These aspects of the studies are also summarised in Table 10.1.

Abutalebi, Miozzo, and Cappa (2000) reported a case with subcortical polyglot aphasia. The participant had Armenian as her L1 and started learning English at the age of 4 at school. As an adult, she learned Italian informally after settling in Italy. She had kept using all three languages actively and retained high proficiency in all of them. After a left-hemispheric stroke, with the lesion localised in the left caudate nucleus in the basal ganglia, she developed non-fluent aphasia that comparably affected all three languages, and also started spontaneously switching from one language to the other. As a result, monolingual interlocutors could not understand her. She was fully aware of her impairment. In a three-language naming task, 


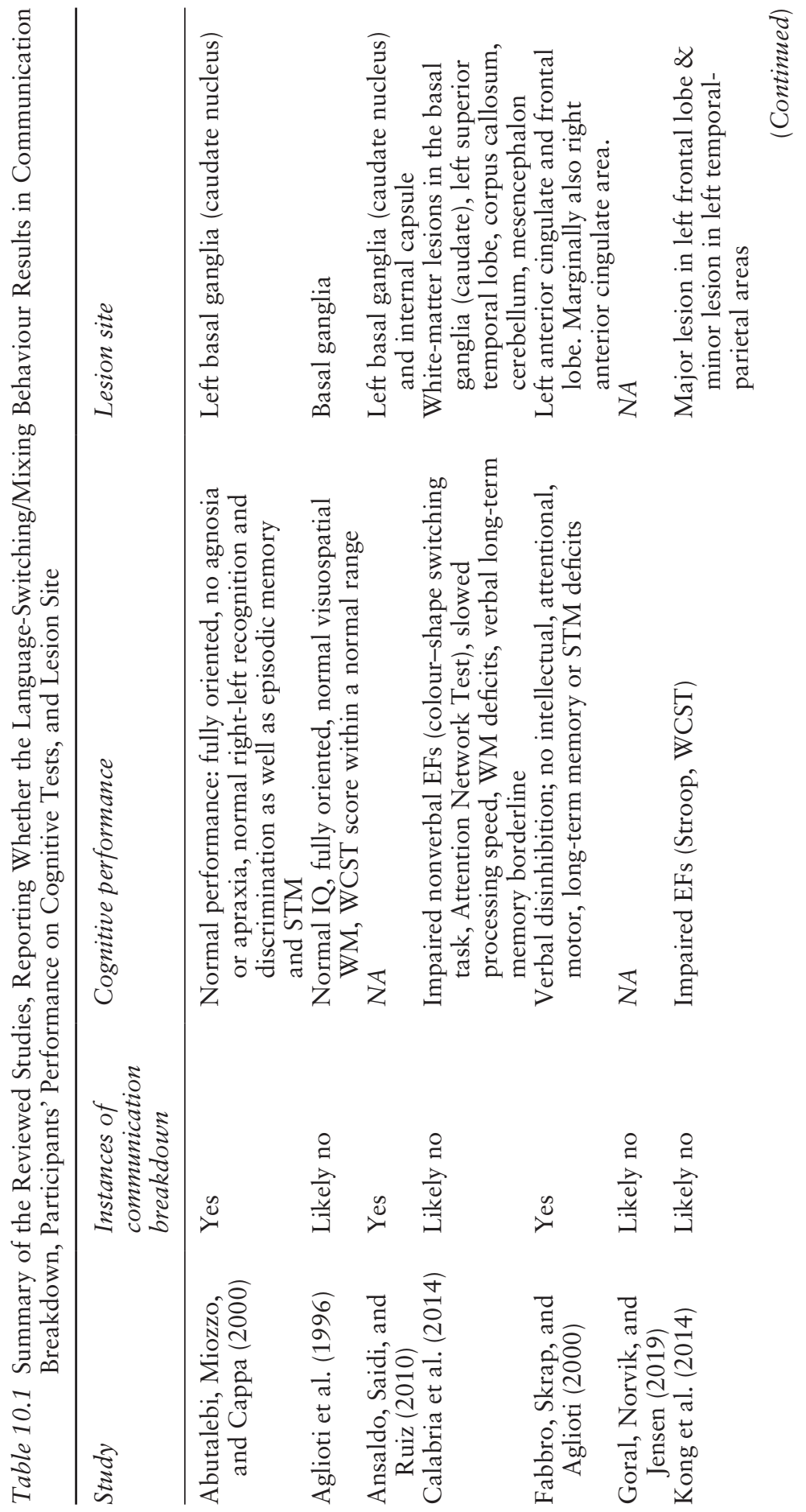




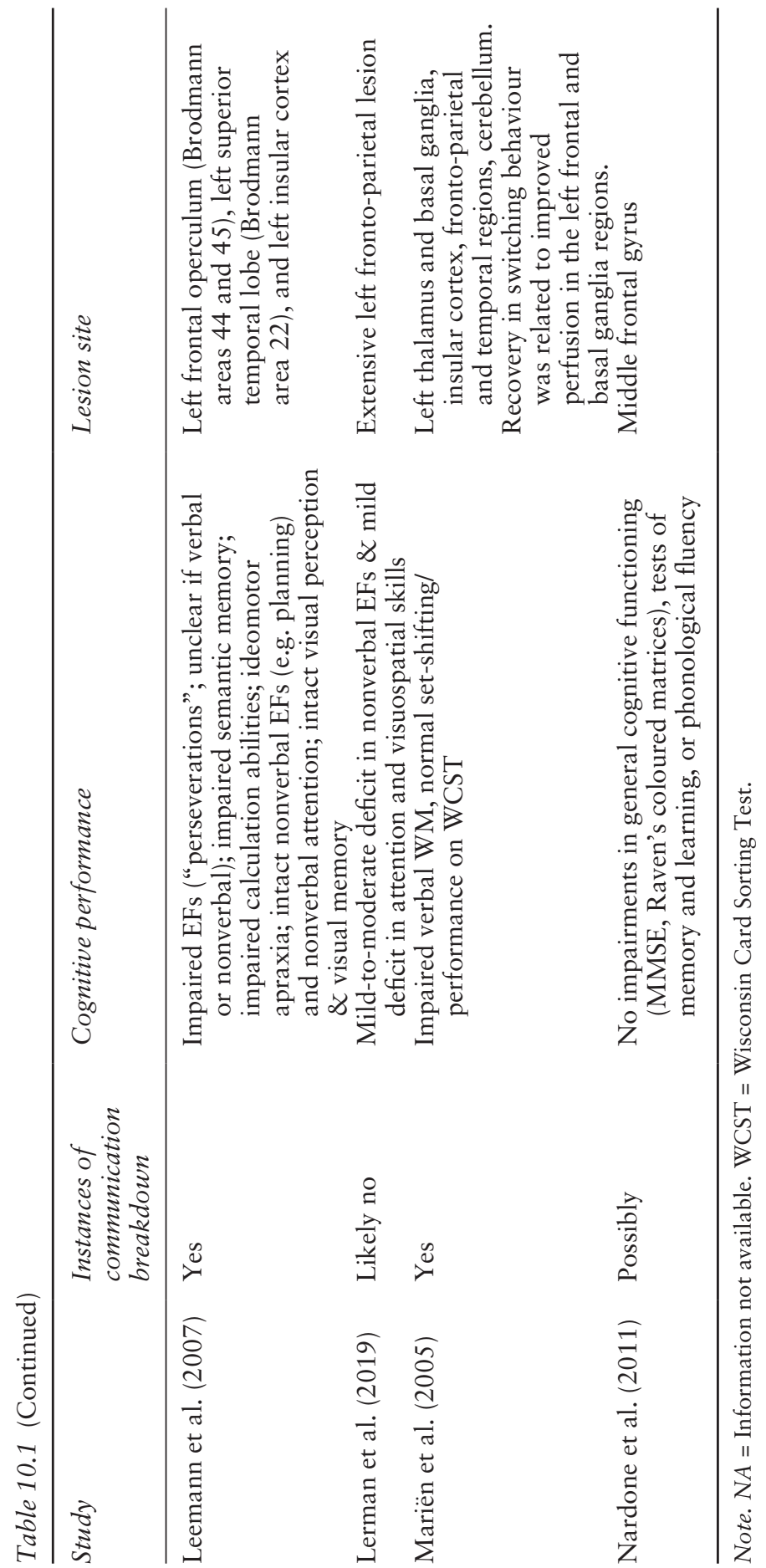


she demonstrated a mild naming deficit but with particular difficulties in answering in the required language. The mixing was present to a similar extent in all three languages, and the direction of mixing varied. However, she never replaced English or Italian output with Armenian output (L1 but also the least used language). The participant seemed to have correct word names available in different languages during a session, as she was able to produce the correct word names but at an improper time, i.e. in a non-target language (for example, she produced "orologio" when the English word for "clock" was required, and "clock" when the Italian word "orologio" was required). The authors interpreted this finding as indicating a dysfunctional control mechanism. Given that the mixing behaviour took place in genuine monolingual contexts (i.e. in settings where the interlocutors of the participant were monolingual), we also interpret this participant as being a case of true pathological mixing. In a neurological examination, the participant did not show any impairments: she was fully oriented in time and space, showed no signs of apraxia or agnosia, and had normal short-term memory (STM). There is no explicit mention on e.g. inhibition or set-shifting tasks specifically.

Aglioti et al. (1996) presented a case of a bilingual person with strokeinduced aphasia who had subcortical lesions mainly in the basal ganglia. After brain damage, her mother tongue, Venetian, was more deficient than the less practised language, standard Italian. In sessions with a VenetianItalian bilingual speech-language therapist, the participant had difficulties keeping to the language of the session, particularly during the L1 (Venetian) sessions, even whenever reminded not to switch to Italian. A neurological examination revealed normal performance not only in tests of intelligence and visuospatial working memory (WM), but also in the Wisconsin Card Sorting Test (WCST), reflecting ability to switch between visual sorting rules. Thus, in this case the language-switching deficit reflects an underlying control deficit, and the latter must be language-specific. However, since the interlocutor during the sessions was Venetian-Italian bilingual, one cannot rule out that the use of Italian was a compensatory strategy to circumvent the deficient first language abilities. The participant also had more difficulties when translating into her L1 than L2.

Ansaldo, Saidi, and Ruiz (2010) described a bilingual person with transcortical mixed aphasia as a result of a stroke that caused a subcortical lesion in the left internal capsule and the left caudate nucleus. This participant was a native Spanish speaker who had learned some English in childhood and later used it every day when residing in the USA, including for reading and studying. His symptoms included anomia in both languages as well as "compulsive" language-switching in monolingual communicative situations (i.e. in settings in which the examiners were monolingual). According to the authors, this involuntary switching usually led to communication breakdown, and although the participant was aware of the switching deficit, he 
was not able to control it. Attempts to control the switching resulted in slowing down of speech production. We also interpret this switching pattern as a case of pathological switching. Unfortunately, there is no report of the performance of this participant in EF tasks.

Calabria et al. (2014) reported on a participant without aphasia but with Multiple Sclerosis, who also demonstrated suspected pathological switching. She was a highly educated 44-year-old Catalan-Spanish bilingual whose first language was Catalan and who had started learning Spanish at the age of 5 . She had started showing unusual and nonpredictable language-switching with her daughter, and this behaviour was also noted by her neurologist. In the neuropsychological assessment, the participant also frequently switched to Spanish when speaking in Catalan, despite the fact that the examiner kept speaking in Catalan. The examiner, however, was a Catalan-Spanish bilingual and performed the testing of both languages, so communication breakdown likely did not occur. The participant showed cross-language intrusions in an experimental language-switching task, especially when switching to her first language. As action naming was more impaired in the first than second language, it remains uncertain whether the switching behaviour could have at least partly reflected word-retrieval difficulties and whether the participant exhibited truly pathological switching by the present criteria. She, however, showed impaired performance in two nonlinguistic EF tasks (a switching task and the Attention Network Test), indicating that the putative language selection deficit also extended to nonverbal domains. The lesions of the participant were located in the left temporal lobe and the basal ganglia, as well as some other subcortical structures.

Fabbro, Skrap, and Aglioti (2000) described a bilingual person with brain damage (resulting from a tumour) whose L1 was Friulian and L2 Italian. This participant had a lesion primarily affecting the left anterior cingulate and areas of the left frontal lobe, and marginally involving the right anterior cingulate area. Neuropsychological evaluation revealed no intellectual, attentional, motor, long-term memory, or STM disorders. He displayed, however, "verbal disinhibition," a term that presumably refers to lack of inhibition in the verbal modality. According to a neurolinguistic evaluation, the participant did not demonstrate language-mixing or any other language impairment, but he only exhibited notable language-switching, as he often alternated between Friulian and Italian across different utterances. Although he had been instructed to speak in only one language (Italian on day/session 1 and Friulian on day/session 2), he did not manage to inhibit his compulsive tendency to alternate between his two languages across utterances. $\mathrm{He}$ was always aware of the examiner's instructions, as he often commented on his switching behaviour or apologised for it. Thus, one could assume that this participant's switching pattern was unintentional, but his intact monitoring system was able to note the errors. It could also be argued, however, that, since the examiner was a Friulian-Italian bilingual speaker, 
the participant presumably knew that the examiner could understand both Friulian and Italian, and so resorted to language-switching to enhance communication efficiency. Under this assumption, the participant could have used language-switching as a "communicative strategy" (see Goral, Norvik, and Jensen 2019), and therefore his switching behaviour cannot necessarily be characterised as pathological. However, he exhibited the same switching behaviour even when he addressed people who could not understand Friulian (e.g. hospital staff in Trieste). Since language-switching in this context resulted in communication breakdown, we argue that this participant exhibited instances of both non-pathological language-switching (e.g. during his interactions with the examiner) and pathological language-switching (e.g. during his interactions with the hospital staff). Although his neuropsychological assessment did not tap into nonverbal inhibition, and given that he showed no signs of memory limitations and was able to detect errors in his behaviour, one could assume that his pathological language-switching behaviour partly stemmed from control problems. The fact that he displayed verbal disinhibition suggests that not all aspects of his language control abilities were fully intact.

Goral, Norvik, and Jensen (2019) examined language-mixing behaviour in aphasia. They did not make a distinction between mixing and switching but used these terms interchangeably. Goral et al. analysed connected language production elicited from 11 multilingual persons with stroke-induced chronic aphasia. Different combinations of languages were represented in this sample of PWA. Most of the PWA were tested on all of their languages. Data collection took place in relatively monolingual settings in two countries (Norway, USA) in that all examiners avoided language-mixing or switching. However, the "participants knew that the examiners or the interpreters ... spoke at least two if not all of each participant's languages" (Goral, Norvik, and Jensen 2019, 920). The authors reported production data from a personal narrative and from a picture sequence description. Participants with more severe aphasia exhibited a mixing/switching behaviour more frequently than those with milder aphasia. Furthermore, testing participants in their weaker language resulted in increased languagemixing/switching as compared to testing them in their strongest language. According to Goral et al., these two pieces of evidence support the idea that PWA use language-mixing or switching as a strategy to cope with wordfinding difficulties (anomia). Since the mixing/switching behaviour attested in Goral et al.'s participants with aphasia appears to be largely voluntary and controlled, and given that none of them switched to a language not known to their interlocutors (and thus communication breakdown never occurred), we agree with the authors that none of their participants with aphasia exhibited pathological code-switching/mixing. Goral et al. did not report data on naming, cognitive control or memory abilities of their participants, nor did they provide information on their lesion sites. 
Lerman et al. (2019) provided a more detailed analysis of a Hebrew-English bilingual participant reported in Goral, Norvik, and Jensen (2019). This participant developed non-fluent aphasia following a stroke that resulted in an extensive fronto-parietal lesion in the left hemisphere. Neuropsychological assessment showed a mild-to-moderate deficit in EF, measured by nonverbal subtests (Symbol Cancellation, Clock Drawing, Symbol Trails, Design Memory, Mazes, Design Generation) of the Cognitive Linguistic Quick Test (Helm-Estabrooks 2001), and a mild deficit in attention and visuospatial skills. This participant was also administered tasks tapping into naming abilities and language production at the sentence and discourse level in both Hebrew and English. The participant exhibited an asymmetric mixing/switching pattern, as significantly more languagemixing/switching took place when the target language was the participant's weaker language, English, than when it was his stronger language, Hebrew. The authors argued that this asymmetric pattern partly stemmed from lexical retrieval difficulty, which was greater in his weaker than stronger language. Lerman et al.'s claim that their participant's mixing/switching behaviour was due to lexical retrieval problems was further supported by the fact that he predominantly exhibited language-mixing (i.e. alternation of English and Hebrew within utterances), not language-switching (i.e. alternation of English and Hebrew across utterances). Just like Goral, Norvik, and Jensen (2019), Lerman et al. (2019) argued that their bilingual participant used language-mixing as a strategy to maximise communication. The authors also reported that, although Hebrew and English were examined in monolingual contexts, the experimenters were Hebrew-English bilinguals. Hence, the participant's mixing/switching behaviour never led to communication breakdown, and, therefore, based on the diagnostic of pathological mixing/switching we employ here his mixing/switching behaviour was not pathological.

Kong et al. (2014) reported of a trilingual (Cantonese-English-Mandarin) 77-year-old female speaker who developed fluent aphasia following a traumatic brain injury. Testing revealed that she had Wernicke's aphasia in all three languages. This participant had a major lesion in the left frontal lobe and a minor lesion in the left temporal-parietal areas. The authors also reported data from a healthy control participant. A modified version of the Stroop colour-word test and the WCST revealed impaired EFs and perseveration errors for the PWA. This patient produced unintelligible neologisms and jargon, and also demonstrated a severe deficit in lexical retrieval during a spontaneous speech task. Moreover, while being tested, she frequently switched between her three languages during conversation, which resulted in reduced comprehensibility. The authors also examined their participants' language-switching/mixing behaviour in confrontation naming and discourse production in all of their three languages. The two participants had to name pictures in Cantonese, English, and Mandarin, as well as to converse with one of the authors on various topics associated 
with these pictures on separate days for each language. The control participant rarely-to-never switched to a non-target language. This was not the case for the PWA, however. Language-mixing/switching in Cantonese (L1) was more prominent in discourse production than in confrontational naming for the same lexical items. Kong et al. (2014) adopted the view that language-switching/mixing can vary as a function of the amount of stress in the environment (Javier and Marcos 1989), and suggested that connected speech poses more cognitive load on the neural system than confrontation naming. Although Kong et al. (2014) referred to their patient's mixing/ switching behaviour as "pathological," they did not state whether the participant knew that their interlocutor/examiner could understand all of her languages. We assume that this was the case, as the same examiner elicited discourse productions in all three languages. Therefore, based on our diagnostic criterion for the presence of pathological mixing/switching, the patient's mixing/switching behaviour could not be considered pathological.

Leemann et al. (2007) reported on a PWA who exhibited paradoxical switching to a barely-mastered and hardly ever used second/foreign language. This participant was an 89-year-old man, a native speaker of French, who had lived in the French-speaking part of Switzerland for all his life. He learned German at school for seven years and also attended a course in German as an adult. However, he hardly ever spoke German in his life, as he only spoke French at work and with his family. He suffered a stroke resulting in a lesion in the left frontal operculum (Brodmann areas 44 and 45), left superior temporal lobe (Brodmann area 22), and left insular cortex. Initially he had global aphasia, and the authors also reported impaired EFs ("perseverations"; the authors do not report whether the task was verbal or nonverbal) and calculation abilities, impaired semantic memory, and ideomotor apraxia. However, the participant had intact aspects of nonverbal EF (e.g. planning) and intact attention, as measured by the Rey-Osterrieth Complex Figure Test (Osterrieth 1944), as well as intact visual perception and intact visual memory. Almost a month after his stroke, the participant started responding to questions and making spontaneous comments. Surprisingly, most of his speech was in German. Importantly, this was the case even in his interactions with his wife, who did not speak German. A systematic investigation of his language abilities took place two months post-onset. No switching was observed when the participant was required to repeat simple words and simple utterances in French and German. However, when he answered everyday life questions asked in French or in German (by native speakers of French or German) during different "French-speaking sessions" and "German-speaking sessions," he switched from French to German (in a French context) to a significantly larger extent than from German to French (in a German context). His switching behaviour, thus, was unidirectional. The authors did not explicitly report if the examiners could understand both French and German or if the participant knew which languages the examiners could understand. It appears, however, that at least in some other 
contexts of interaction this participant exhibited pathological languageswitching/mixing, e.g. using German when talking to his wife. Also, the fact that he resorted to his weaker language supports this interpretation. An alternative explanation could be that this unidirectional switching behaviour could stem from a non-parallel language impairment. That is, it may be that, as a result of his stroke, French was affected much more than German, and thus his switching pattern could be interpreted not as pathological per se but as reflecting stroke-induced asymmetrical language impairment. We believe, however, that, even if this were the case, the fact that he used German when he talked to his wife, who could not understand German, could also suggest deficient cognitive control, e.g. difficulties monitoring the situation and the language being used.

Mariën et al. (2005) reported of a 10-year-old bilingual boy with subcortical aphasia. He was an early bilingual, using English (L1) with his parents and Dutch (L2) with friends and at school. He suffered two strokes, after which he showed symptoms of fluent aphasia in both languages, such as empty output, perseverations, and semantic errors. He also exhibited very prominent, spontaneous language-mixing and switching in both directions, also in conversations with monolingual interlocutors. He mixed the two languages at phonological, morphological, lexical, and syntactic levels. As his switching/mixing behaviour appears spontaneous and not strategic and took place in monolingual situations, it can be considered pathological. MRI measurement four months after the second stroke revealed damage in subcortical structures such as the left thalamus and the basal ganglia, in addition to the insular cortex. SPECT measurement also revealed left-hemispheric perfusion defects in the fronto-parietal and temporal regions and in the thalamus, basal ganglia, and cerebellum. Cognitive testing after the second stroke revealed that verbal WM was impaired, whereas visual setshifting (measured with the WCST) was normal. Some other EFs that would be of interest here could not be reliably assessed as the participant's other incapacities affected his performance on the Stroop task (inhibitory control) and the Trail Making Test (set-shifting). Six months after the second stroke, the pathological switching and mixing behaviour ceased, but the participant still showed impaired naming scores in L2 and a deviant pattern of errors in naming in both languages in comparison to a control group. According to the authors, the participant used language-switching/mixing at this late stage to overcome word-finding difficulties in a controlled and conscious manner, in contrast to the spontaneous switching and mixing present at an earlier stage. SPECT revealed a re-perfusion of the left frontal cortex and left basal ganglia, but a remaining perfusion deficit in the thalamus and left temporo-parietal areas. This pattern suggests that a network encompassing frontal and basal ganglia regions had a central role in the original pathological switching and mixing behaviour of the participant.

Nardone et al. (2011) presented a case of a bilingual speaker who suffered a stroke that affected the left middle frontal gyrus. German was his L1, and 
he had started learning Italian at the age of 6 . After the stroke, he showed a compulsive tendency to produce utterances that alternated between the two languages. During neurolinguistic assessment, he was aware that he had to speak in only one language, but he nevertheless often switched to the other language and tended to apologise for it. The examiner was a German-Italian bilingual, and it is not reported whether the participant was aware of this fact. Therefore, it is not clear whether his switching behaviour could at least partly reflect him knowing that the examiner could understand both languages, and therefore it cannot with certainty be categorised as pathological. Neuropsychological tests measuring cognitive functioning (Mini Mental State Examination, Rey's Auditory Verbal Learning Test, Immediate visual memory, Raven's Coloured Matrices, Constructive praxis, Phonological verbal fluency) did not reveal impairments. Interestingly, repetitive excitatory TMS on the left dorsolateral prefrontal cortex led to a significant increase of utterances in the appropriate language, and inhibitory TMS on the same region increased utterances in the inappropriate language. This study provides evidence for the role of the left dorsolateral frontal region in language control. Table 10.1.

\section{Discussion and Conclusions}

The present narrative review focused on language-switching and -mixing patterns exhibited by bilingual/multilingual speakers with brain damage, with somewhat varying aetiologies but with special emphasis on stroke patients with aphasia. We addressed three questions: 1) Does excessive language-switching and/or -mixing behaviour exhibited by bilingual/multilingual speakers with brain damage constitute a communicative strategy reflecting deficient linguistic resources that are due to brain damage (e.g. Goral, Norvik, and Jensen 2019; Grosjean 1985), or do they reflect deficient cognitive control mechanisms needed in effective bilingual language use (see Green 1998, for a theoretical summary)? 2) What is the relationship between participants' switching/mixing patterns and cognitive control abilities? 3) What is the neural basis (brain correlates) of pathological languageswitching and -mixing?

With respect to Question 1, we see that some of the patterns of language-switching/mixing exhibited by PWA could be interpreted as reflecting a strategy of the participants to maximise communication, as they suffered from deficient linguistic abilities, such as word-retrieval problems (e.g. Aglioti et al. 1996; Goral, Norvik, and Jensen 2019; Kong et al. 2014; Lerman et al. 2019). Importantly, however, there were also cases where this interpretation was not likely, as language-switching/mixing appeared to be unintentional and beyond the control of the participant. In such cases, switching/mixing was interpreted as being pathological and reflecting difficulties in language selection and control. For example, the behaviour of the participant reported by Abutalebi, Miozzo, and Cappa (2000), who during 
the same session produced translation equivalents of target words in her different languages but at inappropriate times, appears as relatively compelling evidence for an impairment of control, instead of deficient linguistic abilities. Also, participants who kept switching and/or mixing in genuinely monolingual communicative situations, which resulted in communication breakdown, suggest that switching/mixing is not always serving a communicative purpose (see especially Ansaldo, Saidi, and Ruiz 2010; Abutalebi, Miozzo, and Cappa 2000; Mariën et al. 2005).

It was not always possible to obtain certainty about the nature (pathological vs. non-pathological) of the language-switching/mixing behaviour exhibited by the multilingual speakers with brain damage. Particularly in cases where the examiner was multilingual (see, for example, Fabbro, Skrap, and Aglioti 2000; Kong et al. 2014), one could only speculate on whether that had affected the switching/mixing behaviour of the participant. Such cases do not provide optimal settings to distinguish whether the switching is pathological, as the examiner can understand all the languages of the participant and communication breakdown can never occur as a result of language-switching/mixing, thus allowing its use as a communicative strategy. We therefore propose that researchers investigating language-switching/mixing patterns should carefully consider situational factors that might affect participants' strategies during the testing situation, as these factors may obscure the possibilities to diagnose pathological language-switching/ mixing properly. To distinguish between the two possibilities (control deficit vs. communicative strategy), examiners/experimenters in a testing situation would ideally be monolingual with respect to the language being studied, and participants should be explicitly (and repeatedly, if needed) informed about that. It has to be acknowledged, however, that in many testing situations it is hard to follow the recommendation above, as most people living in a given country are familiar with the "majority language." In addition to controlling the testing situation to the extent possible, not only language test results (e.g. results from tasks tapping lexical retrieval in different languages) but also broader neuropsychological and experimental testing can provide hints on the nature of participants' deficits.

To address Question 2, we scrutinised the participants whose switching/mixing behaviour appears to reflect a control impairment and reviewed whether their pathological language-switching/mixing was accompanied by impaired EFs/cognitive control as measured by EF tasks. As domain-general inhibitory control is assumed to be central to the process of switching between languages (see e.g. Declerck and Philipp 2015, for a review), we expected that participants demonstrating pathological switching should also show defective performance on tasks of inhibition, both verbal and nonverbal. If nonverbal inhibition (tapped, for example, by the Simon task) were found impaired in participants exhibiting pathological language-switching/ mixing, this would provide evidence for the view that domain-general cognitive control is engaged in bilingual language control. The same logic 
applies to other EFs assumed to be important in language-switching, such as set-shifting and monitoring processes. As many of the currently reviewed studies do not include tasks tapping into nonverbal and verbal EFs such as those above, we urge future research to address this more systematically.

Nevertheless, despite the large variation and limits in the reported measures, some initial observations can be made. For example, the child participant of Mariën et al. (2005) showed true pathological switching based on our analysis, but the performance in a set-shifting measure that is largely nonverbal (WCST) was reported to be normal. Furthermore, Leemann et al.'s (2007) case, who exhibited some uncontroversial instances of pathological switching, had intact aspects of nonverbal EF (e.g. planning) and intact nonverbal attention. The participant of Fabbro, Skrap, and Aglioti (2000), who also exhibited some clear instances of pathological switching, showed defects in verbal inhibition. The participants of Kong et al. (2014) and Calabria et al. (2014) showed problems in both linguistic and nonlinguistic tasks requiring cognitive control; however, the communicative situation of the testing was (at least to some extent) bilingual and they could thus not be with certainty categorised as cases of pathological switching by the present criterion. While there is a lot of uncertainty with these few cases, it appears that pathological switching/mixing can sometimes be accompanied by rather well-functioning nonverbal EFs (Mariën et al. 2005; Leemann et al. 2007) but perhaps not as likely by intact verbal EFs (Fabbro, Skrap, and Aglioti 2000; Mariën et al. 2005). This would mean that the control processes for language-switching/mixing are more similar with languagerelated EFs than they are with nonverbal EFs. Pathological switching would thus not necessarily be caused by damage to domain-general control processes. Instead, the processes of controlling language selection are perhaps at least partly distinct and not fully shared across domains. This would be consistent with some of the few studies on aphasia that explicitly investigated the relationship between language control and domain-general control and found evidence for their separability (Dash and Kar 2014; Gray and Kiran 2016). Even though no clear conclusions can be drawn from the set of data discussed here, this analysis demonstrates the potential of using patient cases for providing complementary insights into the relationship between language control and domain-general cognitive control.

Finally, to address Question 3, we paid attention to the brain correlates of our distinction between pathological vs. non-pathological switching/ mixing, to see whether there is consistency in damage location of the cases demonstrating pathological switching (by our definition) in particular, and to what extent these brain areas correspond to the regions known to be relevant for domain-general cognitive control. Interestingly, inspection of the summary of the lesion site information for participants exhibiting pathological vs. non-pathological switching (see Table 10.1) shows that the few cases of clear and predominant pathological switching (Abutalebi, Miozzo, and Cappa 2000; Ansaldo, Saidi, and Ruiz 2010; Mariën et al. 2005) all 
demonstrate subcortical damage, and particularly in the basal ganglia. For the participants in whom true pathological switching did not look likely or in whom it could not be verified, the pattern of lesions seems more variable, encompassing frontal, temporal, and parietal cortical regions. Notably, a recovery from symptoms of pathological switching in the participant of Mariën et al. (2005) was associated with improved perfusion in left frontal and basal ganglia regions, whereas this measure did not improve in the other regions originally damaged, i.e. temporal, parietal areas, and the cerebellum. This suggests that, in addition to the left frontal cortex, the basal ganglia play a critical role in efficient language selection and switching (as proposed by e.g. Abutalebi and Green 2007; Abutalebi, Miozzo, and Cappa 2000; Green and Abutalebi 2013; see also Adrover-Roig et al. 2011, among others), whereas the other, cortical regions (especially the posterior ones) may be related to the persisting language processing deficits observed in the participant. What remains to be delineated is to what extent bilingual language control networks and nonlinguistic cognitive control networks are overlapping and shared in the brain (see Calabria et al. 2018, for a recent review).

To conclude, Goral, Norvik, and Jensen (2019) make an important point in not categorising all switching/mixing present in bilingual PWA as "pathological" when switching/mixing in fact aims to serve a communicative purpose (see also Grosjean 1985). While we conclude that pathological switching/mixing, defined as a language control deficit, appears to exist, we also argue that future studies should be clearer on this distinction, and, in fact, carefully analyse (and test) whether the switching patterns reflect an underlying cognitive control deficit or a communicative strategy. What is also evident from this review is that, to date, surprisingly few studies on language-switching/mixing in PWA have included systematic testing of EFs, both verbal and nonverbal, in their case reports. Testing multilingual PWA with tasks that have been used to study switching in healthy participants could also elucidate the nature of switching processes. The commonly used picture naming setup with language-switching would allow us to study switching difficulties of brain-damaged speakers in a more controlled situation, and experimental EF tasks could complement the more traditional neuropsychological testing (for such examples, see e.g. Calabria et al. 2014, Dash and Kar 2014; Grunden et al. 2020). A better understanding of cognitive control in language-switching opens up important views on language control mechanisms and the extent they overlap with domain-general cognitive control.

\section{Notes}

1 The term aphasia refers to language/communication impairment due to brain damage, which usually results from a stroke. 
2 In this section, the papers presented and discussed are placed in alphabetical order with one exception: right after Goral, Norvik, and Jensen's (2019) study we present Lerman et al.'s (2019) study (and not Kong et al.'s), because Lerman et al. focus on one of Goral, Norvik, and Jensen's participants, providing a detailed analysis of his linguistic and cognitive performance.

3 Please note that this paper is not meant to be an exhaustive review of all reported cases of language switching and mixing in aphasia. Instead, our aim is to highlight a variety of cases which we think could contribute to the debate on pathological switching/mixing and to the relationship between domain-general EFs and language control. The vast majority of the cases covered here have been diagnosed with aphasia as a result of stroke.

\section{References}

Abutalebi, J. and D.W. Green. 2007. "Bilingual Language Production: The Neurocognition of Language Representation and Control." Journal of Neurolinguistics 20 (3): 242-275. doi:10.1016/j.jneuroling.2006.10.003

Abutalebi, J., A. Miozzo, and S.F. Cappa. 2000. "Do Subcortical Structures Control 'language selection' in Polyglots? Evidence from Pathological Language Mixing." Neurocase 6: 51-56. doi: 10.1080/13554790008402757

Adrover-Roig, D., N. Galparsoro-Izagirre, K. Marcotte, P. Ferre, M.A. Wilson, and A. I. Ansaldo. 2011. "Impaired L1 and Executive Control After Left Basal Ganglia Damage in a Bilingual Basque-Spanish Person with Aphasia.” Clinical Linguistics \& Phonetics 25: 480-498.

Aglioti, S., A. Beltramello, F. Girardi, and F. Fabbro. 1996. "Neurolinguistic and Follow-Up Study of An Unusual Pattern of Recovery from Bilingual Subcortical Aphasia.” Brain 119: 1551-1564. doi:10.1093/brain/119.5.1551

Albert, M.L. and L.K. Obler. 1979. The Bilingual Brain. New York: Academic Press.

Ansaldo, A.I., L.G. Saidi, and A. Ruiz. 2010. "Model-driven Intervention in Bilingual Aphasia: Evidence from a Case of Pathological Language Mixing.” Aphasiology 24: 309-324. doi:10.1080/02687030902958423

Bhat, S. and S. Chengappa. 2005. "Code Switching in Normal and Aphasic Kannada-English Bilinguals." In Proceedings of 4th International Symposium on Bilingualism, edited by J. Cohen, K. McAlister, K. Rolstad, and J. McSwan, 306-316. Somerville, MA: Cascadilla Press.

Bialystok, E. 2017. "The Bilingual Adaptation: How Minds Accommodate Experience." Psychological Bulletin 143 (3): 233-262. doi:10.1037/bul0000099

Bialystok, E., F.I.M. Craik, D.W. Green, and T.H. Gollan. 2009. "Bilingual Minds.” Psychological Science in the Public Interest 10 (3): 89-129. doi:10.1177/1529 100610387084

Calabria, M., A. Costa, D.W. Green, and J. Abutalebi. 2018. "Neural Basis of Bilingual Language Control." Annals of the New York Academy of Sciences. Advance online publication. doi:10.1111/nyas.13879

Calabria, M., P. Marne, L. Romero-Pinel, M. Juncadella, and A. Costa. 2014. "Losing Control of Your Languages: A Case Study." Cognitive Neuropsychology 31 (3): 266-286. doi:10.1080/02643294.2013.879443

Dash, T. and B.R. Kar. 2014. "Bilingual Language Control and General Purpose Cognitive Control Among Individuals with Bilingual Aphasia: Evidence Based on Negative Priming and Flanker Tasks." Behavioural Neurology 2014: 679706. doi:10.1155/2014/679706 
De Baene, W., W. Duyck, M. Brass, and M. Carreiras. 2015. "Brain Circuit for Cognitive Control is Shared by Task and Language Switching." Journal of Cognitive Neuroscience 27 (9): 1752-1765. doi:10.1162/jocn_a_00817

de Bruin, A., B. Treccani, and S. Della Sala. 2015. "Cognitive Advantage in Bilingualism: An Example of Publication Bias?” Psychological Science 26 (1): 99-107. doi:10.1177/0956797614557866

Declerck, M. and A.M. Philipp. 2015. "A Review of Control Processes and their Locus in Language Switching." Psychonomic Bulletin and Review 22 (6): 16301645. doi:10.3758/s13423-015-0836-1

Fabbro, F. 1999a. The Neurolinguistics of Bilingualism: An Introduction. Hove, England: Psychology Press/Taylor \& Francis.

Fabbro, F. 1999b. "Aphasia in Multilinguals." In Concise Encyclopedia of Language Pathology, edited by F. Fabbro, 335-340. Oxford, UK: Pergamon Press.

Fabbro, F., M. Skrap, and S. Aglioti. 2000. "Pathological Switching between Languages After Frontal Lesions in a Bilingual Patient." Journal of Neurology, Neurosurgery, \& Psychiatry 68: 650-652. doi:10.1136/jnnp.68.5.650

Gardner-Chloros, P. 2009. Code-switching. Cambridge, UK: Cambridge University Press.

Genesee, F. 1989. “Early Bilingual Development: One Language or Two?” Journal of Child Language 16 (1): 161-179. doi:10.1017/S0305000900013490

Goral, M., M. Norvik, and B.U. Jensen. 2019. "Variation in Language Mixing in Multilingual Aphasia." Clinical Linguistics \& Phonetics 33: 915-929. doi:10.10 80/02699206.2019.1584646

Gray, T. and S. Kiran. 2016. “The Relationship between Language Control and Cognitive Control In Bilingual Aphasia.” Bilingualism: Language and Cognition 19 (3): 433-452. doi:10.1017/S1366728915000061

Green, D.W. 1998. "Mental Control of the Bilingual Lexico-Semantic System." Bilingualism: Language and Cognition 1: 67-81. doi:10.1017/S13667289980 00133

Green, D.W. and J. Abutalebi. 2013. "Language Control in Bilinguals: The Adaptive Control Hypothesis.” Journal of Cognitive Psychology 25 (5): 515-530. doi:10. 1080/20445911.2013.796377

Green, D.W., A. Grogan, J. Crinion, N. Ali, C. Sutton, and C.J. Price. 2010. "Language Control and Parallel Recovery of Language in Individuals with Aphasia.” Aphasiology 24 (2): 188-209. doi:10.1080/02687030902958316

Green, D.W. and L. Wei. 2014. “A Control Process Model of Code-switching." Language, Cognition and Neuroscience 29: 499-511. doi:10.1080/23273798.2 014.882515

Grosjean, F. 1985. "Polyglot Aphasics and Language Mixing: A Comment on Perecman (1984)." Brain and Language 26: 349-359. doi:10.1016/0093-934x(85)90048-3

Grunden, N., G. Piazza, C. García-Sánchez, and M. Calabria. (2020). "Voluntary Language Switching in the Context of Bilingual Aphasia." Behavioral Sciences 10 (9): 141. doi:10.3390/bs10090141

Helm-Estabrooks, N. 2001. Cognitive Linguistic Quick Test. San Antonio, TX: The Psychological Corporation.

Javier, R.A. and L.R. Marcos. 1989. "The Role of Stress on the LanguageIndependence and Code-Switching Phenomena." Journal of Psycholinguistic Research 18: 449-472. 
Jylkkä, J. 2017. "Bilingual Language Switching and Executive Functions.” Doctoral dissertation, Åbo Akademi. http://www.doria.fi/bitstream/handle/10024/147587/ jylkka_jussi.pdf? sequence $=2$

Jylkkä, J., M. Lehtonen, A. Kuusakoski, F. Lindholm, S.C.A. Hut, and M. Laine. 2018a. "The Role of General Executive Functions in Receptive Language Switching and Monitoring." Bilingualism 21 (4): 839-855. doi:10.1017/S1366728917000384

Jylkkä, J., M. Lehtonen, F. Lindholm, A. Kuusakoski, and M. Laine 2018b. "The Relationship Between General Executive Functions and Bilingual Switching and Monitoring in Language Production.” Bilingualism: Language and Cognition 21: 505-522. doi:10.1017/S1366728917000104

Kong, A.P.H., J. Abutalebi, K. S-Y. Lam, and B. Weekes. 2014. "Executive and Language Control in the Multilingual Brain.” Behavioural Neurology 2014. doi:10.1155/2014/527951

Lanza, E. 1992. “Can Bilingual Two-year-olds Code-switch?” Journal of Child Language 19: 633-658.

Lanza, E. (1997) 2004. Language Mixing in Infant Bilingualism: A Sociolinguistic Perspective. Paperback edition with new Afterword. Oxford: Oxford University Press.

Leemann, B., M. Laganaro, V. Schwitter, and A. Schnider. 2007. "Paradoxical Switching to a Barely-Mastered Second Language by an Aphasic Patient.” Neurocase 13: 209-213. doi:10.1080/13554790701502667

Lehtonen, M., A. Soveri, A. Laine, J. Järvenpää, A. de Bruin, and J. Antfolk. 2018. "Is Bilingualism Associated with Enhanced Executive Functioning in Adults? A Meta-Analytic Review.” Psychological Bulletin 144 (4): 394-425. doi:10.1037/ bul0000142

Lerman, A., L. Pazuelo, L. Kizner, K. Borodkin, and M. Goral. 2019. "Language Mixing Patterns in a Bilingual Individual with Non-Fluent Aphasia." Aphasiology 33: 1137-1153. doi:10.1080/02687038.2018.1546821

Luk, G., E. De Sa, and E. Bialystok. 2011. "Is There a Relation Between Onset Age of Bilingualism and Enhancement of Cognitive Control?" Bilingualism: Language and Cognition 14: 588-595. doi:10.1017/S1366728911000010

Luk, G., D.W. Green, J. Abutalebi, and C. Grady. 2012. "Cognitive Control for Language Switching in Bilinguals: A Quantitative Meta-Analysis of Functional Neuroimaging Studies." Language and Cognitive Processes 27 (10): 1479-1488. doi:10.1080/01690965.2011.613209

Mariën, P., J. Abutalebi, S. Engelborghs, and P.P. De Deyn. 2005. “Pathophysiology of Language Switching and Mixing in an Early Bilingual Child with Subcortical Aphasia.” Neurocase 11: 385-398. doi:10.1080/13554790500212880

Meuter, R.F.I. and A. Allport. 1999. "Bilingual Language Switching in Naming: Asymmetrical Costs of Language Selection." Journal of Memory and Language 40: 25-40. doi:10.1006/jmla.1998.2602

Myers-Scotton, C.M. (1993) 1997. Duelling Languages: Grammatical Structure in Codeswitching. Oxford: Oxford University Press.

Moritz-Gasser, S. and H. Duffau. 2009. "Cognitive Processes and Neural Basis of Language Switching: Proposal of a New Model.” NeuroReport 20: 1577-1580. doi:10.1097/WNR.0b013e328333907e

Nardone, R., P. De Blasi, J. Bergmann, F. Caleri, F. Tezzon, G. Ladurner, S. Golaszewski, and E. Trinka. 2011. "Theta Burst Stimulation of Dorsolateral 


\section{Valantis Fyndanis and Minna Lebtonen}

Prefrontal Cortex Modulates Pathological Language Switching: A Case Report.” Neuroscience Letters 487: 378-382. doi:10.1016/j.neulet.2010.10.060

Osterrieth, P.A. 1944. "Le test de copie d'une figure complexe: Contribution à l'étude de la perception et de la mémoire." Archives of Psychology 30: 286-356.

Paap, K.R., H.A. Johnson, and O. Sawi. 2015. "Bilingual Advantages in Executive Functioning Either Do Not Exist or Are Restricted to Very Specific and Undetermined Circumstances." Cortex 69: 265-278. doi:10.1016/j.cortex.2015. 04.014

Paplikar, A. 2016. "Language-mixing in Discourse in Bilingual Individuals with Non-Fluent Aphasia." Unpublished doctoral dissertation, CUNY Graduate Center.

Paradis, M. 1977. "Bilingualism and Aphasia." In Studies in Neurolinguistics, Vol. 3, edited by H. Whitaker and H.A. Whitaker, 65-121. New York: Academic Press.

Soveri, A., A. Rodriguez-Fornells, and M. Laine. 2011. "Is There a Relationship Between Language Switching and Executive Functions in Bilingualism? Introducing a within-group Analysis Approach.” Frontiers in Psychology 2: 1-8. doi:10.3389/fpsyg.2011.00183

Verreyt, N., M. De Letter, D. Hemelsoet, P. Santens, and W. Duyck. 2013. "Cognate Effects and Executive Control in a Patient with Differential Bilingual Aphasia." Applied Neuropsychology: Adult 20: 221-230. doi:10.1080/09084282.2012. 753074

Weissberger, G.H., C.E. Wierenga, M.W. Bondi, and T.H. Gollan. 2012. "Partially Overlapping Mechanisms of Language and Task Control in Young and Older Bilinguals.” Psychology and Aging 27(4): 959-974. doi:10.1037/a0028281 


\section{Part IV}

\section{Concluding Remarks}




\title{
11 Carving New Spaces in the Study of Individual Multilingualism across the Lifespan
}

\author{
Lourdes Ortega
}

Throughout her fruitful career, Elizabeth (Liz) Lanza has succeeded admirably in carving new spaces for others to join in with the study of multilingualism. One way she has done this is by offering her own individual work as an invitation and bid for more research, an invitation that many others have felt thrilled to accept. This modus operandi had already started in 1990, with her doctoral dissertation study. There Liz crafted deep, original ideas, which continued flourishing into acclaimed early solo publications such as "Can Bilingual Two-Year-Olds Code-switch?" (in the Journal of Child Language, 1992) and her seminal book Language Mixing in Infant Bilingualism: A Sociolinguistic Perspective (with Oxford University Press in 1997). Her solo publications have been sustained over four decades of tireless engagement with these and a myriad other research questions pertaining to multilingual children and adults.

But as early as four years out of her dissertation, Liz also began coauthoring and collaborating with vibrant colleagues, many of them peer junior researchers in the Scandinavian context. This is another, equally effective, way in which Liz has opened new research spaces for others to inhabit and make their own: by creating unprecedented synergies for research collaboration. It is therefore only natural, albeit a labor of patient love and extraordinary work, that Liz would conceptualize MultiLing, the Center for Multilingualism in Society across the Lifespan, and then lead it to the phenomenal success it has been as a Center of Excellence, funded by the Research Council of Norway for a full ten years of existence (two maximum renewable cycles since 2013).

The present volume celebrates Liz's field-shaping contributions with ten chapters that showcase central questions about multilingualism and multilinguals that she has led and facilitated over four decades, addressed within psycholinguistic and sociolinguistic lenses, and pertaining to language acquisition, family language planning, and the consequences of aphasia and dementia on language use. I will offer here a commentary of each chapter, which I hope presents readers with a useful retrospective journey of what they have just read and help them further appreciate the lessons learned. 


\section{Lourdes Ortega}

\section{Linking Young Bilingual Children's Development to Discourse Dynamics and Socialization Practices}

For the first time, Lanza (e.g., 1990, 1992, 1997) richly demonstrated that it is in the careful study of unfolding discourse where researchers can understand how different bilingual children are exposed to and interact in their languages in different ways, depending on their home and family contexts. In this way, she showed the unique value of drawing links between discourse and socialization with young children's bilingual development outcomes. The first three chapters in the present collection explore extensions and implications of this micro-level attention to parent-child discourse over the past four decades.

Taking to heart the reality that children are socialized into language practices differently across different local contexts, Simonsen and Southwood (Chapter 1) argue for the central role of cultural context in deciding how to exercise "mindfulness when constructing, using and interpreting child language assessment tools" (p. 25). Comparing the hurdles and solutions in their own work with children and parents in Norway vs. South Africa, they make the important point that, while thinking of socioeconomic differences and differences in language exposure has become routine and sound part of developing good multilingual assessment tools and interpreting the evidence they yield meaningfully, a pending task is to engage with radically different kinds of contexts for child multilingualism, particularly in the Global South. Simonsen and Southwood do not pretend to have all the solutions for how to integrate culture-appropriate language socialization findings, where they exist, into assessment tool development. But they invite readers to ponder over the point made over a decade ago by psychologists Henrich, Heine, and Norenzayan (2010) that much if not all research knowledge in the social sciences is biased and skewed because it is largely grounded in Western, educated, industrialized, rich, and democratic (WEIRD) samples. In addition, they wisely remind readers that there is no such thing as a universal non-WEIRD context, nor a universal WEIRD context, when it comes to language socialization ... or anything else! Nevertheless, their geopolitically diverse experiences with multilingual assessment development in South Africa and Norway have taught them that developing non-WEIRD tools for assessing the diverse multilingualism of children is a high tall order, but an imperative for the field. Inspired by their wise discussion, I am tempted to modify the witty acronym of WEIRD into WWEEIRD, as it seems the development of multilingual assessment tools - and indeed the whole study of multilingualism - will also need to work ways to escape from White-centric and English-centric assumptions and practices.

De Houwer and Nakamura (Chapter 2) set as their goal to evaluate any accumulated empirical support of Lanza's $(1990,1997)$ proposed link between parental discourse strategies and bilingual child outcomes. They admirably uncover and review a database of two family studies and 20 
observational studies of dyadic parent-child interactions with children aged 1 to 12 published in these 40 years. They conclude that Lanza's discourse strategies do seem to matter in fostering bilingual outcomes in the early development of bilingual children. They also tentatively suggest their predictive power may weaken at later ages, as it is well-known that once children enter school many of them will develop a strong preference for the societal language, that is, if schooling is in the societal language, and this new preference may wash out any parental discourse influences. Logically, what may work with 2 -year-olds may work differently with 10-year-olds. For early ages, nevertheless, De Houwer and Nakamura see sufficient evidence to conclude that the parental strategies discovered by Lanza $(1990,1997)$ can be used as a sort of intervention to destabilize intergenerational language loss and reignite a pre-schooler's use of the minority language at home with at least one parent. And yet, by reviewing bilingual parenting advice books published since 2000 (22 in English and 11 in another 6 languages), they made a chilling discovery: Only 6 of 33 books mentioned the discourse strategies or Lanza's bilingual family interaction model, two without really endorsing them as effective. Their review is nuanced, and they emphasize (as did Lanza in her original work) the bidirectionality of interactions: parents can shape children's language choice preferences, but children also change parents, in mutual cycles of influence. A big take-away from De Houwer and Nakamura's chapter is the need to consider discourse strategies with a developmental perspective that assumes they will somewhat change in purpose, role, and effectiveness as the children grow older and develop full agency. In terms of future research, the authors call for more evidence from multi-party family interactions (e.g., as exemplified by Venables, Eisenchlas, and Schalley 2014), so the combined role of all caretakers can be examined, beyond discourse strategies with one parent at a time.

Lanza's pioneering work showed that turn-by-turn co-constructed language choice crystallizes into the preferred code-switching and code-mixing practices that different bilingual children display in interaction with their parents. Phillips and Deuchar (Chapter 3) issue an interesting invitation to go beyond parents and consider communities as well. They note that, as part of developing full functioning membership into bilingual speech communities, bilingual children need to master the code-switching practices of their surrounding adults. They thus tackle the question of whether and how early bilingual children acquire these normative code-switching practices. Their longitudinal evidence is drawn from 84 hours of parent-child conversation by seven children born in Wales, UK, and studied between the ages of 1 and 3 in the CHILDES CIG1 corpus (Aldridge et al. 1998). They can be assumed to be growing up surrounded by adult Welsh-English bilingual speakers, who tend to speak Welsh with each other with a density of about $10 \%$ of intraclausal code-switches to English, as established empirically in the Siarad adult corpus (Deuchar, Webb-Davies, and Donnelly 2018). This type of community represents $20 \%$ of the population of Wales and 


\section{Lourdes Ortega}

is concentrated geographically in the north and west areas of the country. Phillips and Deuchar's analyses show that the overwhelming majority of children's codeswitched utterances in the CIG1 corpus were found to mirror the preferred pattern found in the Siarad adult corpus: English words and phrases appeared inserted into a Welsh morphosyntactic frame. They thus argue that young children code-switch because their parents and their communities code-switch. In terms of how early socialization into the community norms for code-switching may take place, they found that in this bilingual Welsh context children are already mirroring the code-switching patterns of their community at the very early ages of $1 ; 9$ up to $2 ; 6-$ much earlier than the suggested age of 3 found in previous research. Phillips and Deuchar imbue Lanza's seminal findings with a community-wide insight: bilingual children are socialized into code-switching norms just as they are socialized into language.

\section{Wider Societal Perspectives: Policy, Ideology, and Power}

Lanza's seminal work has greatly contributed to the development of a subfield in the study of bilingualism known as family language policy (FLP), and defined as parents' or caregivers' conscious planning of language use among family members at home (King and Fogle 2013; Lanza 2020; Spolsky 2012). The field of FLP has gradually expanded its lens as it has sought to explicate the influences through which parents implicitly and explicitly settle on their own family language policies. Three chapters in the collection help put FLP in a wider societal perspective, connecting what parents do or do not do with policies, ideologies, and systems of power.

King and Curdt-Christiansen (Chapter 4) trace the influence of Lanza's work on the evolving field of FLP at three levels. First, many studies have followed Lanza's example by focusing on the parent-child negotiation of language choice on the fly. But even at this micro level, the FLP lens easily captures the importance of collective ideologies that circulate within communities and in society. This is exemplified in this chapter with CurdtChristiansen's unpublished data that suggest in Singapore, under the tremendous pressure of English, many minority-language affirming discourse strategies by parents and grandparents are being transformed into teachinglike sequences. Namely, adults issue clarification questions and comprehension checks, and they explicitly ask their 3- to 7-year old children to repeat vocabulary they provide to them in the home languages (Mandarin, Malay, and Tamil). Lanza's seminal work has had a second level of influence, King and Curdt-Christiansen note, in fostering the ethnographic study of broader bilingual practices and socialization. In this strand of FLP research, discourse analysis in the micro (family) sphere connects with the constructs of identity, ideology, and agency, which are shaped by realities in the meso (educational) and macro (national context) spheres (e.g., Lanza 2020). Here an example is given of a Chinese family in the UK who is experiencing a 
marked shift to English-dominant family communication in the face of fears that the children will underperform in their (majority-language) schooling. Yet, the data also show a high degree of agency in the bilingual youth asserting Mandarin for virtual transnational communication with the older generation of Mandarin-monolingual grandparents. According to King and Curdt-Christiansen, Lanza has had a third level of influence on the study of FLP, with a more critical lens that helps recognize the political dimensions of family language policy, which stem from constraining, negative ideologies of nationhood, patriotism, and xenophobia. These can deeply and insidiously affect family language policy decisions. In the remainder of the chapter, King presents an unpublished analysis of social media in the US during the Trump presidency. She denounces an "unrecognized nowin, double-bind" for Spanish-speaking immigrant-background parents in this context (p. 98). Parents are torn apart by three contradictory messages heavily circulating in public and social media: to use Spanish as a badge of pride and/or defiance, to support Spanish bilingualism as a means to economic mobility, and to hush Spanish for fear of being identified, attacked, and deported. In sum, in this chapter, King and Curdt-Christiansen help readers refocus Lanza's discourse strategies at three interconnected levels where close micro dynamics and maximal macro societal influences meet and must be understood in the comprehensive study of FLP.

Widening the scope of FLP even more radically, Anthonissen and Stroud (Chapter 5) employ a South African lens that confronts readers with families that fit neither nuclear nor extended traditional structures and are instead characterized by disruption and vulnerability. The authors highlight the implicit, unreflective, and covert nature of much of the "planning" (if the name really applies) that migrant families in South Africa are able to do with respect to the language choice and practices in the family: "Without a stable income, a fixed address - how does one start a family, how does one plan for the future of children, what kind of family intimacy is possible?" (p. 109). Among their informant parents, those who enjoy less precarious family economies have managed to maintain use of their languages in the home (e.g., Kibembe and Swahili for Jacob, a highly skilled tailor) while proudly noting their children were also ably adding the language of schooling (English). But for the parents in families with the most precarious economies, Anthonissen and Stroud conclude "planning" family language choices is a luxury the adults cannot "plan" or "manage." Language and language family policies were low in the priority of concerns. Across the board, all participants "appeared not to think of themselves in terms of multilinguals with 'linguistic capital'" (p. 117). Families whose rich multilingualism includes no or only limited competence in English (e.g., émigrés from Francophone DRC, Burundi, or Rwanda; or from Lusophone Mozambique or Angola) were caught by surprise by the dire expectations for English and experienced their multilingualism as much less advantageous than families whose multilingualism included English (e.g., émigrés from Zimbabwe, 
Malawi, Tanzania, or Nigeria). Nevertheless, it was clear to the researchers that competence in English could make equally qualified immigrants attain more job security (Jacob vs. Claudette, both French-educated DRC skilled tailors). The grim conclusion is: "you cannot plan for the future unless you have one, and you cannot plan for equitable futures when you do not have the means - or access to the means (infrastructures, institutions) - to make them happen" (p. 120). In sum, in Chapter 5, Anthonissen and Stroud make it clear that researchers of FLP must carefully consider social vulnerability in the contexts they investigate. Provisionality, uncertain futures, and temporality are three forces that they argue must be included in future FLP studies of socially and economically vulnerable migrant families in the Global South.

The various multilingualisms lived by families in Chapters 4 and 5 were an outcome of migration flows and mobility across geopolitical borders. But for many bilingual and multilingual families, bi/multilingualism is the outcome of the experience of Indigeneity and colonial occupation and violence, where borders are drawn or redrawn on peoples who are autochthonous to the land, rather than people uprooting and crossing borders. (Nevertheless, global forces of internal and external migration are not alien to many individuals and families in Indigenous communities, e.g., Wyman 2013.) Comparing the contexts for multilingualism of Indigenous peoples in Australia and Norway, Lane and Wigglesworth (Chapter 6) interrogate family language policies and practices in the context of Indigenous language awakening, revitalization, and reclamation. They argue for the importance of three distinct, yet inextricably interconnected spheres of influence. The first sphere is the daily lives of families, where language choices and practices modulate intergenerational transmission of the Indigenous language at the most concrete and material level. The second sphere is educational systems, which depending on governments and historical and political moments can play radically different roles. Educational systems very often serve as tools of colonial control. Perhaps the most horrid example are the boarding schools Lane and Wigglesworth discuss for the Aboriginal and Torres Strait Islander peoples in Australia and the Sámi population in Northern Norway. Nowadays illegal under United Nations international law, these boarding schools were the norm not only in Australia and Norway but also in Canada and the United States from the late 1860s and up to World War II, at least. In better historical times, education systems can become tools for language awakening and revitalization, if they make space for the teaching of (and additionally, better yet, in) the Indigenous languages. Lane and Wigglesworth suggest education can be the space for the socialization of youth not only into their ancestral languages but also into their communities' Indigenous ways of knowing, learning, and teaching. The third sphere - interconnected with these first two of family and education - is made up of language policies, explicitly or implicitly formulated, that force Indigenous communities to abandon their own languages 
and instill linguistic insecurity as an internalized ideology, making them feel ashamed of their languages as inferior. It is through the process of explicit and implicit language policies emotionally eroding generation after generation that parents end up believing the replacement of the home language for the societal majority language is for the good of their children's future. It is through the emotionally wrenching workings of policies, too, that elderly persons may feel ashamed of not speaking their own language well. The similarities in the issues faced by Indigenous communities in Australia and Norway, despite the many historical and geopolitical differences, are uncanny. They are also a good reminder of the many benefits accrued from opportunities for research interaction and collaboration across disparate geographies, such as the ones Liz Lanza has skillfully fostered at MultiLing.

\section{Understanding and Supporting Multilingual Communication for Adults' Well-Being: Health Frailties in Older Age}

Many lay and expert people have become used to the statement that the majority of the world is multilingual. It is perhaps more surprising for many, although not less true, that the world is also aging. In fact, the wellbeing of older adults, defined as 65 or older, will become one of the most important challenges of the 21 st century in any world context. The latest statistics and projections by the United Nations, Department of Economic and Social Affairs, Population Division (2020) estimate that in 2019, 1 in 11 people or $9 \%$ of the world population were older adults. The areas of the world with more older adults at the time of this writing are Eastern and South-Eastern Asia (261 million), followed by Europe and North America (over 200 million). Countries are considered "super-aged" when more than one in five people (or $20 \%$ of the population) are aged 65 or older. Japan and Germany are already super-aged at the time of this writing. By 2030 there will be 34 countries that are super-aged, including the USA, the UK, France, and Singapore. Chapters 7 and 8 in this volume examine questions about language and the well-being of older adults. In both chapters multilinguals' human agency is an important value to nurture.

What health care barriers might language create for multilinguals at an older life stage when, paradoxically, they need to be served by the health system better than ever? $\mathrm{Ng}$ and Cavallaro (Chapter 7) provide a window into the linguistic needs of Chinese-speaking older adults negotiating the health care system of Singapore. As the authors point out, the case of Chinese Singaporean is particularly poignant in the unique multilingual ecology of Singapore. This is the ethnic majority group in the country (74\%, vs. Malays who represent $13 \%$ and Indians, who represent 9\%). Yet, older Chinese Singaporeans are finding it increasingly more difficult to access health care in their own mother tongue, in their own country. This is due to the workings of two language-related mandates. One is the sway of English as an international lingua franca, which has grown dramatically 


\section{Lourdes Ortega}

in Singapore in the last four decades years, making it the preferred and de facto only language for most realms of public life, including healthcare. Mandarin comes second in the language hierarchy of Singapore, given the national demographics. However, the language policy of Speak Mandarin first campaigned in 1979 and an "unabated force" since then (Rubdy 2001, 343) - has persuaded new generations of ethnic Chinese Singaporeans to abandon their own home dialects, for a complex web of ideological reasons that have not gone unchallenged by grassroots movements (Lim, Chen, and Hiramoto 2021). Ng and Cavallaro document the negative consequences these language shifts have had for older Singaporean adults of Chinese ethnic descent, who still primarily speak many Chinese vernaculars - called dialects in Singapore - that are non-mutually intelligible (e.g., Hokkien, Cantonese, Hakka). They find their doctors and nurses use English as the language of health care and have, for the most part, no knowledge of the languages their patients speak. These older adults thus find themselves engaging in language-discordant care, where their doctors and nurses do not share any common language with them. The health care system prioritizes getting by, that is, regularly drawing on $a d$ hoc (untrained) interpretation by family members and occasionally nurses or even staff who happen to speak the language of a given patient. Through interview data, $\mathrm{Ng}$ and Cavallaro reveal how these taken-for-granted practical solutions to language-discordant care both compromise the quality of health care and greatly reduce the ability of older adults to exercise agency over their own health care choices.

Svennevig and Hamilton (Chapter 8) make room for more optimism by asking: How can language be made into a tool that supports high-quality social participation at a time in life when aging persons become more interpersonally isolated? They focus on a subset of older adults in the world, and an important one: persons with dementia. Contrary to myth, dementia can also occur in younger adults. But it is much more frequent among older ages. According to the World Health Organization (2020), between 5\% and $8 \%$ of the world population aged 60 and over has dementia, amounting to 50 million elderly with diagnosed dementia. There are nearly 10 million new cases every year. Little known is that nearly $60 \%$ of the incidence of dementia is located in low- and middle-income countries. Svennevig and Hamilton use data from the two high-income countries where they carry out their work: Norway and the United States. Using Conversation Analysis and Interactional Sociolinguistics, they reveal the architectural resources in turn-by-turn interaction that might create spaces for the elderly person with dementia to participate more agentively and more fully in any interaction. Readers are taken through two multi-party interactions. One interaction is bilingual, in Norwegian and English, at the dinner table. It involves Laura, her husband Gary, and two female friends. Laura is skillfully aided interactionally by her husband. Relying on shared life knowledge, when one of the friends apologizes for overreaching to get some food off the table, Gary invites Laura to tell a childhood story about many cousins stretching arms 
over the large family table to reach rolls. He then manages to support her in telling the story without speaking on her behalf. An example of the subtle resources that Gary uses includes anticipatory laughter, by which he projects for Laura, the teller of the story, and her friends, the recipients of the story, that a "laughable" is about to come in the talk, thus helping Laura stay on track and finish her story. Laura's two friends at the table, by comparison, issue well-intentioned peripheral questions about Laura's family. While their resources are deployed to show interest and make Laura feel included, they instead are "byplay" that each time derails Laura. Interestingly, Svennevig and Hamilton also suggest code-switching from Norwegian to English is detrimental to Laura, at least in this interaction, as it distracts her away from the telling of her childhood story. The other multi-party interaction takes place in the institutional context of an early memory loss support group meeting in the United States. Mary, the dementia expert leading this session, deploys similar resources to those of Gary's and manages to support Jessica in speaking for herself. In sum, in both cases an interlocutor who cared (Gary) or another one who was well trained (Mary) paid vigilant attention to narrative possibilities arising from what the person with dementia might be saying and then oriented to the person's ability to tell something important, despite other visible signs of memory loss. Svennevig and Hamilton's main point is that interactants who learn to support older adults with dementia to speak for themselves, rather than being spoken for, can counter the understandable tendency to withdraw from conversational involvement and to reduce engagement in social interaction, thereby creating favorable conditions for these adults' well-being.

\section{Multilingual Communication in People With Aphasia: The Human and the Theoretical Dimensions}

The last two chapters in the collection are devoted to multilingualism and aphasia, an acquired language disorder resulting from sudden damage to the brain - particularly although not exclusively after a stroke. According to the World Health Organization (n.d.), stroke "is the brain equivalent of a heart attack" and 15 million people worldwide suffer a stroke annually. A third of them die, another third are survivors with permanent disabilities, and the last third are survivors who recover almost completely or with minor impairments. About $30 \%$ of stroke survivors will suffer from aphasia. Half of aphasia cases will show permanent symptoms (half of cases are transient aphasia and will recover after about three months). The prevalence of post-stroke aphasia is greater among adults aged 65 or older than among younger adults (Ellis and Urban 2016), but there is ample evidence that treatments can benefit these older adults with aphasia (Fabian, Bunker, and Hillis 2020). For the elderly adults with dementia studied by Svennevig and Hamilton (Chapter 8), cognitive functioning declines and this makes ideas, thoughts, and memories difficult to access. For people with aphasia, 


\section{Lourdes Ortega}

non-language cognition is intact, and it is only the ability to access ideas and thoughts via language that is problematic; what is affected is the production of speech, and sometimes the comprehension or the ability to read or write as well. However, both multilinguals who suffer dementia and those who suffer aphasia experience communication difficulties, and thus they share the danger of diminished self-confidence and social isolation due to a likely restriction of social interactions. It is therefore of utmost importance to provide high-quality diagnostic and treatment services to multilinguals with aphasia, as increased functioning is possible after proper diagnosis and a suitable choice of treatment for a year or longer.

Norvik and Goral (Chapter 9) discuss the main challenges in diagnosing and treating multilinguals who suffer aphasia, and they offer recommendations for best practices. A striking point is the difficulty yet importance of being able to characterize the proficiency of an individual across their languages prior to the onset of aphasia. Namely, for accurate diagnosis and treatment, aphasia-related qualities of speech must be teased apart from less than "perfect" proficiency qualities of communication that may have already existed for the multilingual patient pre-morbidity. As with all contexts of multilingualism, defining and measuring proficiency in a given language and across languages is therefore a real challenge. Norvik and Goral note that diagnostic accuracy can be increased dramatically by integrating formal and informal, quantitative and qualitative sources of assessment. They must be applied, fundamentally, to all the languages of the multilingual, or at least all that the patient-client wishes to be assessed in. The authors' characterization of the situation for adults with aphasia harkens back to the fundamental issues discussed by Simonsen and Southwood (Chapter 1) for multilingual assessment of children. For example, throughout the chapter, Norvik and Goral emphasize the importance of meaningfully accounting for cultural, linguistic, and social considerations in both the assessments and the treatments offered, and to prioritize the respect for a person's multilingual and multicultural identity. The importance of developing a good knowledge base leading to high-quality services for multilingual persons with aphasia will only grow in the future, as part of a more global growth of multiethnic older groups susceptible to post-stroke aphasia in many world geographies, as attested in the special issue of Aphasiology guest-edited by Centeno, Kiran, and Armstrong (2020).

But multilinguals with aphasia can also provide pieces of the puzzle of a full theory of the human capacity for language, understood to be a capacity that is multilingual, fundamentally and by default. This is ultimately the motivation of Fyndanis and Lehtonen (Chapter 10) in reviewing studies of multilingual persons with aphasia who are reported to engage in higher than expected amounts of language switching or mixing. Their purpose is to elucidate three questions: (1) Is this aphasia symptom the result of uncontrollable and unintentional ("pathological") difficulties at regulating bilingual language use? Or might it be the result - as Goral, Norvik, and Jensen 
(2019) have proposed - of compensatory strategic communication behavior, whereby the multilingual draws from his or her full repertoire in order to ameliorate communication difficulties resulting from the brain damage, such as anomia (i.e., word-finding difficulties)? (2) Is the code-switching or mixing symptom associated with documentation of less than optimal functioning of domain-general cognitive control mechanisms or to deteriorated verbal cognitive control mechanisms? And (3) What are the brain locations for the lesions associated with each patient's aphasia in these cases of higher than expected language switching or mixing?

With regard to the first question, Fyndanis and Lehtonen conclude that in some cases at least the multilinguals knew their interlocutors could not understand the languages they were mixing in their speech, thus attesting to uncontrollable and unintentional (or "pathological") behavior. They do concede that in other attested cases, where the person with aphasia knew their interviewers to share their bilingualism, Goral et al. (2019) may be right in claiming a strategic motivation. As for the second and third questions posed by Fyndanis and Lehtonen in their review, they both indirectly contribute to the currently much scrutinized and disputed cognitive advantages of bilingualism, or the hypothesis that bilinguals develop superior executive control functioning than monolinguals due to their constant juggling with two or more languages (e.g., Antoniou 2019). Part of the wider issue involves the fundamental question of whether language switching behavior relies on domain-general executive functions (which would "train" the brain and result in the putative advantage) or on languagespecific control mechanisms (which would make us expect no general cognitive advantages). Fyndanis and Lehtonen tentatively conclude that there seems to be greater involvement of suboptimal verbal control in the aphasic code-switching and mixing symptom. Indirectly, this may mean that whatever brain gymnastics multilingual code-switching brings about, it would not be expected to result in domain-general cognitive advantages over monolingual brains. This refutation of special cognitive benefits of bilingualism over monolingualism is consistent with several meta-analyses, including one by Lehtonen (Lehtonen et al. 2018). With regard to their Question 3, Fyndanis and Lehtonen note that whenever the location of brain lesions was reported across studies, more often than not there was subcortical damage in the basal ganglia. They thus side with Green and Abutalebi's (e.g., 2013) well-known contention that efficient language selection and switching involves not only the left frontal cortex (i.e., verbal control mechanisms) but also the basal ganglia (i.e., nonverbal cognitive control processes). This might be a point of support in favor of bilingualism having beneficial consequences for cognition. However, definitive answers on any of these issues are far from sight because, as Fyndanis and Lehtonen acknowledge, they hit roadblocks given how few studies report fully and clearly the crucial information points needed to draw firm answers to the three questions they pose. For future studies of multilingual aphasia to 


\section{Lourdes Ortega}

shed useful light on these issues, therefore, Fyndanis and Lehtonen urge researchers to always: (1) report whether the multilingual person with aphasia thought they were speaking to a monolingual or bilingual interviewer; (2) engage in systematic testing of both verbal and nonverbal executive functions; and (3) report the location of the lesion associated with each patient's aphasia.

The reporting standards Fyndanis and Lehtonen propose are most welcomed. In addition, I would like to suggest it will be appropriate to address better in the future the issue of whether language switching or mixing is "pathological" vs. strategic when multilinguals with aphasia engage in such behavior. For one, it has serious consequences for the well-being and treatment of these patients, as a therapist will likely respond to higher than expected code-switching/code-mixing quite differently when the behavior is construed as a deficit versus as a strategy. Moreover, rather than just using an etic (researcher-observer) perspective in deciding the "pathological" versus strategic nature of code-switching and code-mixing by multilinguals with aphasia, ideally these judgments would be made from emic (participant) perspectives, for example, by forming interdisciplinary research teams with members trained in Conversation Analysis and Interactional Sociolinguistics, who can greatly aid in the adjudication of what counts as (in)appropriate communicatively or interactionally, as shown in the study of older persons with dementia by Svennevig and Hamilton (Chapter 8).

\section{Concluding Thoughts}

The authors in this collection have risen to the tall order of celebrating Liz Lanza's seminal influence on scholarship in multilingualism. Across their ten chapters, they clearly show how much Liz's ideas have shaped the field into several fruitful directions over these 40 years. Countless scholars have benefited from her work, by expanding and elaborating on the research spaces that Liz had already carved in 1990 and on the new research paths that she has continued building tirelessly over four decades. Through the original work by Liz, these authors and so many others have forged organic, variegated, and well-traveled pathways for the study of multilingualism over the lifespan, across geopolitical contexts, and inclusive of vulnerable populations. A sociolinguist and qualitative discourse analyst by training, Liz has always been committed to understanding multilingualism as a lived reality for individuals, families, and communities, and as a phenomenon that can be best understood at the confluence of micro and macro levels - from onthe-fly co-constructed discourse to collective policies and ideologies, at the dialectic intersection of systemic structures and human agency. Very few scholars are capable of marrying sociolinguistics and psycholinguistics as Liz has done. In this, too, she stands as an inviting role model for the next generations of scholars of multilingualism. Thank you, Liz Lanza! 


\section{References}

Aldridge, M., R. Borsely, S. Clack, and G. Creunant. 1998. "The Acquisition of Noun Phrases in Welsh.” In Language Acquisition: Knowledge Representation and Processing. Proceedings of GALA '97, edited by C.B. Heycock, R. Shillcock, and A. Sorace. Edinburgh: University of Edinburgh Press.

Antoniou, M. 2019. “The Advantages of Bilingualism Debate." Annual Review of Linguistics 5: 395-415.

Centeno, J.G., S. Kiran, and E. Armstrong. 2020. “Aphasia Management in Growing Multiethnic Populations.” Aphasiology 34 (11): 1314-1455.

Deuchar, M., P. Webb-Davies, and K. Donnelly. 2018. Building and Using the Siarad corpus: Bilingual Conversations in Welsh and English. Amsterdam: John Benjamins.

Ellis, C. and S. Urban. 2016. "Age and Aphasia: A Review of Presence, Type, Recovery and Clinical Outcomes." Topics in Stroke Rehabilitation 23 (6): 430-439.

Fabian, R., L. Bunker, and A.E. Hillis. 2020.” Is Aphasia Treatment Beneficial for the Elderly? A Review of Recent Evidence." Current Physical Medicine and Rehabilitation Reports 8: 478-492.

Goral, M., M. Norvik, and B.U. Jensen. 2019. "Variation in Language Mixing in Multilingual Aphasia." Clinical linguistics \& phonetics 33 (10-11): 915-929.

Green, D.W., and J. Abutalebi. 2013. "Language Control in Bilinguals: The Adaptive Control Hypothesis." Journal of Cognitive Psychology 25 (5): 515-530.

Henrich, J., S.J. Heine, and A. Norenzayan. 2010. “The Weirdest People in the World?" Behavioral and Brain Sciences 33: 61-135.

King, K.A., and L.W. Fogle. 2013. "Research Timeline: Family Language Policy and Bilingual Parenting." Language Teaching 46 (2): 172-194.

Lanza, E. 1990. "Language Mixing in Infant Bilingualism: A Sociolinguistic Perspective." (unpublished Ph.D. dissertation). Washington, DC: Georgetown University.

Lanza, E. 1992. “Can Bilingual Two-year-olds Code-switch?” Journal of child language 19 (3): 633-658.

Lanza, E. 1997. Language Mixing in Infant Bilingualism: A Sociolinguistic Perspective. Oxford, UK: Oxford University Press.

Lanza, E. 2020. "Urban Multilingualism and Family Language Policy." In Urban Multilingualism in Europe: Bridging the Gap between Language Policies and Language Practices, edited by G. Caliendo, R. Janssens, S. Slembrouck, and P.V. Avermaet, 121-139. Berlin: de Gruyter Mouton.

Lehtonen, M., A. Soveri, A. Laine, J. Järvenpää, A. de Bruin, and J. Antfolk. 2018. "Is Bilingualism Associated with Enhanced Executive Functioning in Adults? A Meta-analytic Review." Psychological Bulletin 144 (4): 394-425.

Lim, J. J., S.C. Chen, and M. Hiramoto. 2021. "'You Don't Ask Me to Speak Mandarin, Okay?': Ideologies of Language and Race Among Chinese Singaporeans." Language \& Communication 76: 100-110.

Rubdy, R. 2001. "Creative Destruction: Singapore's Speak Good English Movement." World Englishes 20 (3): 341-355.

Spolsky, B. 2012. "Family Language Policy-The Critical Domain." Journal of Multilingual and Multicultural Development 33 (1): 3-11. 


\section{Lourdes Ortega}

United Nations, Department of Economic and Social Affairs, Population Division. 2020. World population ageing 2019: Highlights. ST/ESA/SER.A/444.

Venables, E., S.A. Eisenchlas, and A.C. Schalley. 2014. "One-parent-one-language (OPOL) Families: Is the Majority Language-speaking parent Instrumental in the Minority Language Development?" International Journal of Bilingual Education and Bilingualism 17 (4): 429-448.

World Health Organization. September 21, 2020. Fact Sheets, Dementia. https://www. who.int/news-room/fact-sheets/detail/dementia

World Health Organization. n. d. The Atlas of Heart Disease and Stroke. https://www. who.int/cardiovascular_diseases/resources/atlas/en/

Wyman, L.T. 2013. "Indigenous Youth Migration and Language Contact." International Multilingual Research Journal 7 (1): 66-82. 


\section{Index}

Note: Page locators in bold refer tables.

acquired language disorder 189

acquisition 56

active bilingualism 34, 49, 51

adaptation 202

adaptation process 15

ad-based platforms 98

adult input 63

African migrants 106, 109-111, 120;

African migrancy 106; African

migration 104

Afrikaans 13, 17, 21, 105,

111-112, 114

age of acquisition 16, 19-20, 22

agency 90, 169, 179, 184, 185

aging population $151-152$

alienation 131

Anglophone countries 116

Anindilyakwa 136

anomia 190

anti-immigrant 94

aphasia 189

aphasia 211, 213-214, 217, 219-227

aphasia batteries 191

apraxia of speech 190

Arabic 42

assessment 189

assessment tools 11, 14, 20, 22-23, 25, 191

assimilation 128-130, 132, 134-135

assimilation policies 124, 128, 134, 138

Barnevernet 94

basal ganglia 213-218, 222, 226

Bembe (Kibembe) 111, 113

bilingual acquisition 57

bilingual advantage 98, 210

Bilingual Aphasia Test 192

bilingual children 3

bilingual development 34, 43-44, 51; awareness 41, 43, 45; Bilingual First Language Acquisition (BFLA) 32, 34, 35, 39-40, 49; developmental stage 49 ; intergenerational language maintenance 44

bilingual discourse strategy/strategies 34; Adult Repetition (AR) 37-39, 42, 45; dual-lingual conversations 31, 39; Language-Switching (LS) 33, 37, 39, 42; Move One Strategy (MOS) 33, 37-41, 43, 50-51

bilingual education 132-133, 136

bilingual families: bilingual family interaction 3; Chinese-English 85; English-Gaelic 84; Korean-English 90; Norwegian-American 84;

Russian-Swedish 90

bilingual parenting 44-46, 49-51, 98

bilingualism 209

Bi-SLI 11, 19, 25

boarding schools $126,128,131,134$

border officials 96

brain tumor 190

Bringing Them Home report 125

broker 173

Bulgarian 35, 41

Burundi 112, 116

Cantonese 39, 56

Cape Town 106-107, 110-111, 113

Catalan 21, 35, 38

CHAT 62

child 31-34, 37-41, 45, 49-50

child agency 94

child-centered adaptation 24 
child-directed speech $16,19,24$

childhood multilingualism 12-14, 25

Chinese 35, 40-42, 83, 88, 90

CIG1 63-64, 67, 69

CIG1 corpus 68

citizenship rights 111

CLAN 65, 68

code-mixing 203, 209

code-switching 56, 84, 173, 178, 185, 203, 209; Marathi/English codeswitching 58; Spanish/English codeswitching 58

code-switching patterns 74;

"intraclausal" code-switching 58

cognates 203

cognitive control 209-214, 219, 222-224, 226

collaborative achievement 169-170

colonialism/colonialist/colonialising

124-125, 138

communication breakdown

211-220, 224

communication pattern 92

communicative skills 108, 117

communicative strategy 211-212, 214,

$219,223-224,226$

comparable 201

complexity index 20

The Comprehensive Aphasia Test 191

control deficit 211, 213, 217, 224, 226

conversation analysis 171

COVID-19 99

critical moment 91

crossing borders 106, 238

cross-linguistic 15, 17-18, 21-22

culture 197

Danish 13, 15

de facto language policy 104, 111-112, 150,240

de jure language policy 104

decision-making 91

"default" language 89

dementia 169-172, 178, 180-185

Democratic Republic of Congo (DRC) 106-107, 111, 116-118, 237-238

deportation 97

deportation of immigrants 96

diagnosis 189

discourse strategies 84

discriminated 97

dispossession 125, 129, 130

distributed cognition 169

diversity 110, 118-121, 200 domain-general cognitive control 209-211, 214, 224-226

domain-general control 213, 225

domain-specific cognitive control 213

dominance 60-61

DRC French 111, 117

Durban 107, 113

Dutch 31, 40-41, 43, 45

dynamic nature of proficiency 197

dysarthria 190

ecological validity 22

education 124, 129-133, 137-138

educational systems $124,130-131$, 138-139

emotional ties 94

emplacement 105, 107

English 13-17, 19-20, 31-45, 88-90, 105, 111-118; English proficiency

109, 111, 114-115; L2-English

114,117

epistemic 170-173, 177, 183, 185

estimated preonset abilities 202

executive functions 209; domain-

general EFs 210, 213, 227; nonverbal

EFs 225; verbal EFs 225

explicit attention 89

explicit language planning 106, 116,120

expressed discourse strategies 89

expressed guess 84

false friends 203

family experiences 95

family/families 32-34, 39, 42, 44-45, 49-51

family language policy (FLP) 83, 90

Farsi 36, 42

father 31-32, 37-41

Finnish 21, 41, 46

FLP literature 104, 106, 118; FLP phenomena 104, FLP research 104, 119

fluency 117-118

focus group 111, 115-116

foreigner 91, 111, 115, 117, 151

formal assessment 192

France 107

Francophone African countries 110, 116, 237

Francophone Canada 107

French 35, 38, 42, 45, 62, 111-112, 116-118

futures 5, 107-109, 119-120, 238 
Gaelic 43

Gamilaraay 136

German 35, 37, 39, 45, 62

good parent 97

governmentality 105, 107

grandparents 89-90

Gurindji Kriol 126

healthcare system 148, 151, 164

Hebrew 21

home language practices $83,90,113$

ICE 96

identity 90, 169, 197

ideology 90

ILO Convention 127, 135

impact belief 43, 50

implicit socialization practice 88

indigenous languages $105,110,115$, 129, 132, 134, 136-139

indigenous people/peoples 127-130, $132,135-136$

indigenous ways of knowing 138-139

individual differences 194

informal assessment 192-193

inhibition 210, 213-214, 217-219, 224-225

inhibitory control 210, 212-213, 222, 224

Initiation-Response-Evaluation sequences 89

input $56,74-75$

institutional interaction 178, 184

interactional sociolinguistics 171

interactional trouble 181

interlocutors 203

interpreter(s) 148, 150, 152, 157-159, $165,195,198$; ad hoc interpreters 148-149, 154, 156, 159-164; professional interpreters 148 , 162-164

interview 113-114, 119-121

intraclausal code-switching 57

Inuit 24

Irish 18

isiNdebele 13

isiXhosa 21-23, 105, 111, 114

isiZulu 13

Italian 21, 35-36, 37, 39

itineraries 5, 107, 118

Japanese 35-43, 45

Johannesburg 107
Kaluli 24

Kaurna 136

Kikongo 111

Kirundi 112

Kiswahili 111

Kriol 124, 131

Kurdish 36, 42

Kven 13

laissez-faire policy 85

Land Sales Act of 1902130

language attrition 197

language background questionnaires 194

language choice 31-34, 37-43, 49-50; Convergent Choice Principle (CCP) 31 ; one parent, one language $(1 \mathrm{P} / 1 \mathrm{~L})$ 34, 50; switch 32, 37, 40-41, 43, 50; unilingual utterances 32

language control 210-214, 219, 223-227

language control deficit 213, 226

language development 115

language-discordance 148

language disorders 198

language exposure 18, 23, 25

language history 191

language identity 116

language impairment 11, 18, 20, 22-23, 189

language interaction 18

language-mixing 32, 202, 209-212, 218-220, 222, 226

language modalities 190

language planning and policy 105

language practices 91

language proficiency 202

language reclamation 124, 137-139

language repertoire 111, 117, 120, 165

language resources 104

language rights/linguistic rights 135,137

language selection $211,214,218,223$, 225-226

language shift 124, 126, 131, 134-135

language socialisation practices 21-23, 25

language socialization 83,85

language-switching 209-214, 217-224, 226

language-switching/mixing 211-212, 214, 219-224, 226

language transmission 126, 134, 136, 138 
Lapp Codicil 127

Lari 111

Latino families 84

Latinos 97

Light Warlpiri 126

limited English proficiency 147-148

Lingala 111

lingua franca 13, 112-113, 116

linguistic and cultural adaptions 25

linguistic and cultural equivalence 19

linguistic capital 5, 117, 237

linguistic comparability 21,23

linguistic competition 91

linguistic constructions 201

linguistic diversity 104, 120-121

linguistic insecurity 137

linguistic repertoire 111, 115-116, 178

Linguistically Displaced Older Adults (LDOA) 151, 154

literacy development 115

Lithuanian 21

LITMUS (Language Impairment Testing in Multilingual Settings) 12

LITMUS Cross-Linguistic Lexical Tasks (CLT) 12, 14, 19-25

Lule Sámi 127, 137

Luo 24

Luxembourgish 21

MacArthur-Bates Communicative Development Inventory (CDI) 12, 14-22, 25; CDI I 14, 16; CDI II $14-18$

mainstream language 91

Malawi 106, 109, 112, 116, 238

Malay 83, 88, 90

Maltese 18

Mandarin 36, 39, 41, 88

Matrix Language Frame 58

media 95

media dimensions 94

media platforms 98

memory 177-178, 181, 183-186

migrant 105-111, 115-120; migrant children 109-110, 115; migrant experience 106-107, 109; migrantfamily 104, 118

minimal grasp 84,90

minority children 95

minority languages 91

mixed utterances $32,37,41,60$, 63, 66

mobility 105, 107, 109, 117, 120 monitoring 210, 212, 214, 218 , 222, 225

monolingual discourse strategy/ strategies 33-34; adult repetition (AR) 33; clarification request 32, 37, 41; expressed guess strategy (EGS) 32, 37-44, 49; minimal grasp strategy (MGS) 32, 37-46, 49-50; translation request 32

monolingual mode 200

mother 31-34, 37-43

mother tongues 124, 134

move-on 84

Mozambique 110, 116, 237

multilingual aphasia 211

multilingual clinicians 198

multilingual language assessment 19, 22 multilingual norms 18

multilingualism 104, 110, 116, 119, 127-128, 133

multimodality 92

multiparty interactions 169,179 , 184-185

Murrinhpatha 136

narrative 115,119 ; narrative data 118 ; narrative production 201; narrative scaffolding 169-170, 182; personal experience 169,171

nation/nation state $128-130,138$

nationalistic 94

Netherlands Sign Language (NGT) 61

Ngarrindjeri 136

Nigeria 116, 238

non-pathological 211

non-pathological languageswitching 219

non-societal language (NonSocLang) $34,38-45,49-51$

non-target language 203

nonverbal EF tasks 210

nonverbal inhibition 224

Noongar/Nyungar 136

North Sámi 127, 135, 137

Norway $12-15,95$

Norwegian 12-13, 15, 17, 21, 32-35, 171-178, 185

Norwegian Child Welfare Services Agency 94

Norwegianise 129-130, 133

official language 119, 121

older adults 147, 152, 154, 158, 165 
oppression 124, 128

organized FLP 85

overt language planning 104, 106

parallel or non-parallel patterns 193

parent 32, 40, 45, 50

parental discourse 34, 35, 39-40, 44-46, 49

parental ideology 91

parental input 61

parental narratives 98

parental report 14

parent-child interactions 3,84

parenting 95

parenting blogs 97

pathological 211, 214, 224-226

pathological language-mixing 211 , 218-219

pathological language-switching

211-212, 218-219, 224-225

pathological language-switching/mixing

209, 212, 214, 219-226

performance stability 204

personhood 172

picture-based task 20

Pitjantjatjara 136

Polish 13, 19, 21, 90

political dimensions of FLP 83

Portuguese 40, 111

postmorbid 196

poverty of the stimulus 57

premorbid 191

Pretoria 107

professional preparation 199

prototypical families 110

racial profiling 96

radical hope 117

receptive bilingualism 51

refugee $111,115,121$

reliability 17

repair $169-170,176,177,179-180$

repetition 84

revitalisation 134-137, 139

Russian 42

Rwanda 116, 237

Sámi 13

Sámi Act of 1987 135, 137

Sámi language/s 126-127, 133, 135,137

Sámi peoples 125-127, 135

Samoa 24 self-rating 194

Serbian 21

SES 13, 15-17, 21, 23, 25

Sesotho 17, 24

set-shifting 210, 214, 216, 217 ,

$$
\text { 222, } 225
$$

Setswana 17

shared child-rearing practice 86

shifting 213

Shona 114

Siarad corpus 63,74

Singapore 147, 149-157, 165

Singaporean families 85

Slovak 21

smaller language communities 200

social media 92, 95

socialization/socializing $31,34,37,43$, 45, 50; bilingual family interaction model (BIFIM) 32-34, 38-40, 44-46, 49-51; conversation 32, 39-42, 49; interaction 31-34, 37-40, 42-44, 49-50

societal language (SocLang) 34, 39, 43, $45,49-51$

sociocultural 17, 19, 21

Somali 13, 90

Somalia 118

South Africa 13-17, 21, 23, 104-120

South African English 17, 21

South Sámi 127, 137

Spanish 35, 37, 45, 49, 97

Spanish-speakers/speaking 95, 98

story climax 170, 176

story consociate $170,173,175,177$

story preface $170,173,185$

story prompt $170,172-178,185$

story recipient 170,175

storytelling 169-171, 174, 176-178, 181, 183-185

stroke 190

subjective time 105

superaged 165

support group 170-171, 178-184

Swedish 13, 21, 36, 40-42, 46, 90

Swiss-German 35

Tagalog 35, 37

Tanzania 160, 238

task switching 212-213

temporal regimes 106, 108, 119-120

temporality 104-108, 110, 117, 120,238

translation 201 


\section{Index}

transnational families 94

traumatic brain injury 190

Truth and Reconciliation Commissions 138

Tsimane 24

Turkish 13, 21

Twitter 96

typological differences 19

Ukrainian 40, 43

unreflective FLP 85

US Customs and Border Protection 96

usage-based approach to language acquisition 57

validity 15,17 vocabulary assessment 14,19

vulnerability $105,119,121$, 237-238

Waradjuri 136

Warlpiri 136

WeChat 93

WEIRD contexts 14, 25

well-being 185

Welsh 57, 63

Wolof 24

Wordbank project 18

Xitsonga 21-22

Yolnu Matha 136 